ADVERTIMENT. La consulta d'aquesta tesi queda condicionada a l'acceptació de les següents condicions d'ús: La difusió d'aquesta tesi per mitjà del servei TDX (www.tesisenxarxa.net) ha estat autoritzada pels titulars dels drets de propietat intel-lectual únicament per a usos privats emmarcats en activitats d'investigació i docència. No s'autoritza la seva reproducció amb finalitats de lucre ni la seva difusió i posada a disposició des d'un lloc aliè al servei TDX. No s'autoritza la presentació del seu contingut en una finestra o marc aliè a TDX (framing). Aquesta reserva de drets afecta tant al resum de presentació de la tesi com als seus continguts. En la utilització o cita de parts de la tesi és obligat indicar el nom de la persona autora.

ADVERTENCIA. La consulta de esta tesis queda condicionada a la aceptación de las siguientes condiciones de uso: La difusión de esta tesis por medio del servicio TDR (www.tesisenred.net) ha sido autorizada por los titulares de los derechos de propiedad intelectual únicamente para usos privados enmarcados en actividades de investigación y docencia. No se autoriza su reproducción con finalidades de lucro ni su difusión y puesta a disposición desde un sitio ajeno al servicio TDR. No se autoriza la presentación de su contenido en una ventana o marco ajeno a TDR (framing). Esta reserva de derechos afecta tanto al resumen de presentación de la tesis como a sus contenidos. En la utilización o cita de partes de la tesis es obligado indicar el nombre de la persona autora.

WARNING. On having consulted this thesis you're accepting the following use conditions: Spreading this thesis by the TDX (www.tesisenxarxa.net) service has been authorized by the titular of the intellectual property rights only for private uses placed in investigation and teaching activities. Reproduction with lucrative aims is not authorized neither its spreading and availability from a site foreign to the TDX service. Introducing its content in a window or frame foreign to the TDX service is not authorized (framing). This rights affect to the presentation summary of the thesis as well as to its contents. In the using or citation of parts of the thesis it's obliged to indicate the name of the author 


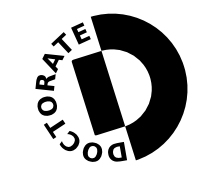

DOCTORAL STUDIES

$$
\begin{gathered}
\text { PhD Thesis } \\
-2014-
\end{gathered}
$$

\title{
CONFORMANCE CHECKING AND \\ DIAGNOSIS IN PROCESS MINING
}

\author{
Jorge Munoz-Gama \\ Advisor \\ Josep Carmona Vargas
}



A thesis presented by Jorge Munoz-Gama

in partial fulfillment of the requirements for the degree of

Doctor per la Universitat Politècnica de Catalunya - BarcelonaTech

$\begin{array}{ll}\text { Author: } & \text { Jorge Munoz-Gama } \\ \text { Address: } & \text { Department of Software } \\ & \text { Edifici Omega, Despatx S108 } \\ & \text { C/ Jordi Girona, 1-3 } \\ & \text { 08034 Barcelona, Spain } \\ & \text { jmunoz@lsi.upc.edu } \\ \text { Email: } & \text { jmunozgama@gmail.com }\end{array}$

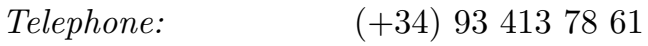



To Loly, Emilio, Alex, and the rest of my family 



\section{Summary}

\section{Conformance Checking and Diagnosis in Process Mining}

In the last decades, the capability of information systems to generate and record overwhelming amounts of event data has experimented an exponential growth in several domains, and in particular in industrial scenarios. Devices connected to the internet (internet of things), social interaction, mobile computing, and cloud computing provide new sources of event data and this trend will continue in the next decades. The omnipresence of large amounts of event data stored in logs is an important enabler for process mining, a novel discipline for addressing challenges related to business process management, process modeling, and business intelligence. Process mining techniques can be used to discover, analyze and improve real processes, by extracting models from observed behavior. The capability of these models to represent the reality determines the quality of the results obtained from them, conditioning its usefulness. Conformance checking is the aim of this thesis, where modeled and observed behavior are analyzed to determine if a model defines a faithful representation of the behavior observed a the log.

Most of the efforts in conformance checking have focused on measuring and ensuring that models capture all the behavior in the log, i.e., fitness. Other properties, such as ensuring a precise model (not including unnecessary behavior) have been disregarded. The first part of the thesis focuses on analyzing and measuring the precision dimension of conformance, where models describing precisely the reality are preferred to overly general models. The thesis includes a novel technique based on detecting escaping arcs, i.e., points where the modeled behavior deviates from the one reflected in log. The detected escaping arcs are used to determine, in terms of a metric, the precision between log and model, and to locate possible actuation points in order to achieve a more precise model. The thesis also presents a confidence interval on the provided precision metric, and a multi-factor measure to assess the severity of the detected imprecisions.

Checking conformance can be time consuming for real-life scenarios, and understanding the reasons behind the conformance mismatches can be an effort-demanding task. The second part of the thesis changes the focus from the precision dimension to the fitness dimension, and proposes the use of decomposed techniques in order to aid in checking and diagnosing 
fitness. The proposed approach is based on decomposing the model in single entry single exit components. The resulting fragments represent subprocesses within the main process with a simple interface with the rest of the model. Fitness checking per component provides well-localized conformance information, aiding on the diagnosis of the causes behind the problems. Moreover, the relations between components can be exploded to improve the diagnosis capabilities of the analysis, identifying areas with a high degree of mismatches, or providing a hierarchy for a zoom-in zoom-out analysis. Finally, the thesis proposed to main applications of the decomposed approach. First, the theory proposed is extended to incorporate data information for fitness checking in a decomposed manner. Second, a realtime event-based framework is presented for monitoring fitness. 


\section{Contents}

$\begin{array}{lr}\text { Summary } & 7\end{array}$

$\begin{array}{llr}\text { I Introduction } & 13\end{array}$

1 Introduction $\quad \mathbf{1 5}$

1.1 Process Mining . . . . . . . . . . . . . . . . . . 17

1.2 Conformance Checking and its Challenges . . . . . . . . . . . . . 19

1.2.1 Dimensions of Conformance Checking . . . . . . . . . . . . . . . 19

1.2.2 Replay-based and Align-based Conformance Checking . . . . . . . . . 21

1.2.3 Challenges of Conformance Checking . . . . . . . . . . . . . . . 22

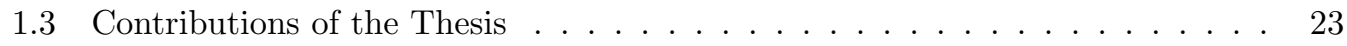

1.3.1 Precision Checking . . . . . . . . . . . . . . . . . . . 24

1.3.2 Decomposed Conformance . . . . . . . . . . . . . . . . . 25

1.4 Structure of the Thesis . . . . . . . . . . . . . . . . 26

1.5 Publications and Thesis Impact . . . . . . . . . . . . . . . . . . 26

2 Preliminaries $\quad \mathbf{2 7}$

2.1 Basic Notations . . . . . . . . . . . . . . . . . . . . . . . . . 27

2.2 Event $\operatorname{Logs} \ldots \ldots \ldots \ldots \ldots \ldots \ldots \ldots$

2.3 Process Models . . . . . . . . . . . . . . . . . . . . . . . . 31

2.4 Process Modeling Formalisms . . . . . . . . . . . . . . . . . . . . . 34

2.4 .1 Petri Nets . . . . . . . . . . . . . . . . . . . . 34

2.4 .2 Workflow Nets . . . . . . . . . . . . . . . . 36

2.4 .3 Other Formalisms . . . . . . . . . . . . . . . . . 36

II Precision in Conformance Checking 39

3 Precision based on the Log 41 
3.1 Introduction . . . . . . . . . . . . . . . . . . . . 41

3.2 The Importance of Precision . . . . . . . . . . . . . . . . . . . . . . . 42

3.3 Related Work . . . . . . . . . . . . . . . . . . . . . . . . . . . . . . . . . . . .

3.4 Precision based on Escaping Arcs . . . . . . . . . . . . . . . . . . . . . . . . . . . . . . . . . . . 45

3.5 Constructing the Observed Behavior . . . . . . . . . . . . . . . . 48

3.6 Incorporating Modeled Behavior . . . . . . . . . . . . . . . . 50

3.7 Detecting Escaping Arcs and Evaluating Precision . . . . . . . . . . . . . . 52

3.8 Minimal Imprecise Traces . . . . . . . . . . . . . . . . . . . . . . . . 56

3.9 Experimental Results . . . . . . . . . . . . . . . . . . . . . . 57

3.10 Limitations and Extensions . . . . . . . . . . . . . . . . 61

3.10 .1 Unfitting Scenario . . . . . . . . . . . . . . . . . . . . . . . . . . . . . . . .

3.10 .2 Indeterministic Scenario . . . . . . . . . . . . . . . . . . . . . . . . . . . . . . . 62

3.11 Conclusions . . . . . . . . . . . . . . . . . . . . 63

4 Qualitative Analysis of Precision Checking $\quad 65$

4.1 Introduction . . . . . . . . . . . . . . . . . 65

4.2 Robustness on the Precision . . . . . . . . . . . . . . . . . . 66

4.3 Confidence on Precision . . . . . . . . . . . . . . . . . . . . . . . . . . . . . . . . . .

4.3.1 Upper Confidence Value . . . . . . . . . . . . . . . . . . . . . . . . . . . . . . . . . 72

4.3 .2 Lower Confidence Value . . . . . . . . . . . . . . . . . . . . . 74

4.4 Severity of an Escaping Arc . . . . . . . . . . . . . . . . . . . . . 74

4.4.1 Weight of an Escaping Arc . . . . . . . . . . . . . . . . . . . 75

4.4.2 Alternation of an Escaping Arc . . . . . . . . . . . . . . . . . 76

4.4.3 Stability of an Escaping Arc . . . . . . . . . . . . . . . . . 77

4.4.4 Criticality of an Escaping Arc . . . . . . . . . . . . . . 78

4.4.5 Visualizing the Severity . . . . . . . . . . . . . . . . 79

4.5 Experimental Results . . . . . . . . . . . . . . . . . . . 80

4.6 Conclusions . . . . . . . . . . . . . . . . . . . . 84

5 Precision based on Alignments $\quad 85$

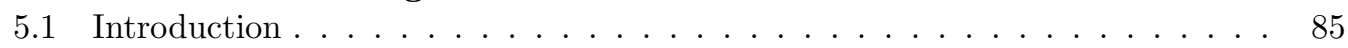

5.2 Cost-Optimal Alignment . . . . . . . . . . . . . . . . . . . . . . . . . . . . 87

5.3 Precision based on Alignments . . . . . . . . . . . . . . . . . . . . . . . . . . . . . . 92

5.3 .1 Precision from 1-Alignment . . . . . . . . . . . . . . . 92

5.3.2 Precision from All-Alignment . . . . . . . . . . . . . . . . . . 96

5.3.3 Precision from Representative-Alignment . . . . . . . . . . . . . . . . . . 98

5.4 Abstractions for the Precision based on Alignments . . . . . . . . . . . . . . 100

5.4.1 Abstraction on the Order . . . . . . . . . . . . . . 101

5.4.2 Abstraction on the Direction ................. 102

5.5 Experimental Results . . . . . . . . . . . . . . . . . . . 106

5.6 Conclusions . . . . . . . . . . . . . . . . . . . . 111 


\section{Decomposition in Conformance Checking}

6 Decomposed Conformance Checking $\mathbf{1 1 5}$

6.1 Introduction . . . . . . . . . . . . . . . . . . . . . 115

6.2 Related Work . . . . . . . . . . . . . . . . . . . . . . . . . . . 116

6.3 Single-Entry Single-Exit and Refined Process Structure Tree . . . . . . . . . . 117

6.4 Decomposing Conformance Checking using SESEs . . . . . . . . . . . . . 119

6.5 Bridging a Valid Decomposition . . . . . . . . . . . . . . . . . . . . . . . . 121

6.5.1 Decomposition with invisible/duplicates . . . . . . . . . . . 127

6.6 Experimental Results . . . . . . . . . . . . . . . . . . . . . . . . . 131

6.7 Conclusions . . . . . . . . . . . . . . . . . . . . . . 137

7 Topological and Multi-level Conformance Diagnosis 139

7.1 Introduction . . . . . . . . . . . . . . . . . . . . . . . . . . . . . . 139

7.2 Topological Conformance Diagnosis . . . . . . . . . . . . . . . . . . . . . . 140

7.3 Multi-level Conformance Diagnosis and its Applications . . . . . . . . . . . . 144

7.3.1 Stand-alone Checking . . . . . . . . . . . . . . . . . . . . . 144

7.3 .2 Multi-Level Analysis . . . . . . . . . . . . . . . . . . . . . . . . . 145

7.3 .3 Filtering . . . . . . . . . . . . . . . . . . . . 145

7.4 Experimental Results . . . . . . . . . . . . . . . . . . . . . . . . . 147

7.5 Conclusions . . . . . . . . . . . . . . . . . . . . . . . . . 148

8 Data-aware Decomposed Conformance Checking 149

8.1 Introduction . . . . . . . . . . . . . . . . . . . . . . . . . . . . 149

8.2 Data-aware Processes . . . . . . . . . . . . . . . . . . . . . . . . . 152

8.2 .1 Petri nets with Data . . . . . . . . . . . . . . . . . . . . . 152

8.2.2 Event Logs and Relating Models to Event Logs . . . . . . . . . . . . . 155

8.2 .3 Data Alignments . . . . . . . . . . . . . . . . . . . . . . . 156

8.3 Valid Decomposition of Data-aware Models . . . . . . . . . . . . . . . 158

8.4 SESE-based Strategy for a Valid Decomposition . . . . . . . . . . . . . . 160

8.5 Implementation and Experimental Results . . . . . . . . . . . . . . . . . . . . 161

8.6 Conclusions . . . . . . . . . . . . . . . . . . . . . . 163

9 Event-based Real-time Decomposed Conformance Checking 165

9.1 Introduction . . . . . . . . . . . . . . . . . . . . . . . . . 165

9.2 Event-based Real-time Decomposed Conformance . . . . . . . . . . . . . . . 166

9.2.1 Model and Log Decomposition . . . . . . . . . . . . . . . . 166

9.2 .2 Event-based Heuristic Replay . . . . . . . . . . . . . . . . . . . . . . . 168

9.3 Case Example and Experimental Results . . . . . . . . . . . . . . . . . . 170

9.3 .1 Description . . . . . . . . . . . . . . . . . . . . . 170

9.3.2 Experimental Scenario Evaluation . . . . . . . . . . . . . . . . . 171

9.3.3 Experimental Comparison . . . . . . . . . . . . . . . . . . . 175 
9.4 Conclusions . . . . . . . . . . . . . . . . . . 176

$\begin{array}{lll}\text { IV Closure } & 177\end{array}$

10 Conclusions $\quad 179$

10.1 Conclusion and Reflection . . . . . . . . . . . . . . . . . 179

10.2 Summary of Contributions . . . . . . . . . . . . . . . . . . . . 180

10.3 Challenges and Directions for Future Work . . . . . . . . . . . . . . 181

$\begin{array}{lc}\text { Bibliography } & 185\end{array}$

$\begin{array}{ll}\text { Publications } & 195\end{array}$

$\begin{array}{lr}\text { Acknowledgements } & 197\end{array}$ 


\section{Part I}

\section{Introduction}





\section{Chapter 1}

\section{Introduction}

There is a wide range of ways to define the concept of process. A simple but intuitive alternative is to define a process as a sequence of activities performed in a specific order to achieve a specific goal. And when this definition is considered one realizes that processes are everywhere in our daily lives. Processes are crucial parts of our industries and organizations. An assembly line in a car plant, or the management of packages in a delivery company are examples of processes in industrial scenarios. However, the concept of process is wider than that, being present in any daily activity: buying groceries in the city marked is, by itself, a process. When you go to a market you visit the different stands in a given order. The stands where you buy may depend on your needs that day (e.g., groceries for yourself or to prepare a big meal for some guests), or even on the date itself (e.g., the butcher closes on Monday). The order of the stalls is determined by a set of factors (e.g., buy in stalls close to each others to avoid crossing the market each time, or buy heavy purchases at the end to not carry them around). Even concurrent activities may appear (e.g., send your son to buy bread while you buy the fish). Hence, buying groceries is a complete process with all its elements. Other examples of processes are the application for a credit card, boarding and security procedures before a flight, or the preoperative steps before a surgical procedure.

The times of paper and ink are progressively passing, and nowadays more and more organizations are supported by some kind of IT system. The support of the IT system in small organizations is usually passive, like the use of simple spreadsheets to keep the accounts of a small business. In other cases, like in banking, this support plays a more active role where each action is strictly controlled and monitored by the IT system. But most the IT systems used in practice have something in common: the possibility to keep some kind of record of the actions occurred during the executions of the process they support. This records are called event logs.

Consider, for example, a doctoral scholarship process of some university. Doctoral students that want to apply for a scholarship submit their academic record, the CV of their 
advisor, and a description of their proposed project. Then the university evaluates the documentation and decides whether they grant the scholarship. The process starts collecting all the documents and starting the procedure (Start Processing). Then, the three documents are evaluated in any order (Evaluate Academic Record, Evaluate Advisor CV and Evaluate Project). Once all documents have been evaluated, a final evaluation is done by the chief of the department (Final Evaluation) and the application is accepted (Accepted) or rejected (Rejected). Finally, the decision taken is notified to the student (Notify Results). Table 1.1 show an example of an event log recorded by the IT system supporting this scholarship process. Each row represents an event of the log, and the events are grouped, not chronologically, but by student - i.e., case identifier - to help on understanding the process.

\begin{tabular}{ccccccc}
\hline Case & Event & Timestamp & Activity & Employee & Student & $\ldots$ \\
\hline 1 & 1 & 01-01-2014 10:00 & Start Processing & Merce & Alex & $\ldots$ \\
1 & 2 & 01-01-2014 11:30 & Evaluate Academic Record & Fernando & Alex & $\ldots$ \\
1 & 4 & 01-01-2014 13:30 & Evaluate Project & Fernando & Alex & $\ldots$ \\
1 & 8 & $01-01-2014$ 17:00 & Evaluate Advisor CV & Fernando & Alex & $\ldots$ \\
1 & 9 & $02-01-2014$ 10:00 & Final Evaluation & Ana & Alex & $\ldots$ \\
1 & 11 & $02-01-2014$ 11:00 & Accept & Ana & Alex & $\ldots$ \\
1 & 12 & $02-01-2014$ 12:00 & Notify Results & Merce & Alex & $\ldots$ \\
\hline 2 & 3 & $01-01-201412: 00$ & Start Processing & Merce & Dolores & $\ldots$ \\
2 & 5 & $01-01-201414: 00$ & Evaluate Academic Record & Maria & Dolores & $\ldots$ \\
2 & 6 & $01-01-201415: 00$ & Evaluate Advisor CV & Maria & Dolores & $\ldots$ \\
2 & 7 & $01-01-201416: 00$ & Evaluate Project & Fernando & Dolores & $\ldots$ \\
2 & 10 & $02-01-201410: 30$ & Final Evaluation & Ana & Dolores & $\ldots$ \\
2 & 13 & $02-01-201413: 00$ & Reject & Ana & Dolores & $\ldots$ \\
2 & 15 & $02-01-201416: 00$ & Notify Results & Merce & Dolores & $\ldots$ \\
\hline 3 & 14 & $02-01-201415: 00$ & Start Processing & Merce & Emilio & $\ldots$ \\
3 & 16 & $02-01-201417: 30$ & Evaluate Academic Record & Maria & Emilio & $\ldots$ \\
3 & 17 & $02-01-201418: 00$ & Evaluate Project & Maria & Emilio & $\ldots$ \\
3 & 18 & $03-01-201410: 00$ & Final Evaluation & Ana & Emilio & $\ldots$ \\
3 & 19 & $03-01-201411: 00$ & Reject & Ana & Emilio & $\ldots$ \\
3 & 21 & $03-01-201413: 00$ & Notify Results & Merce & Emilio & $\ldots$ \\
\hline 4 & 20 & $03-01-201412: 00$ & Start Processing & Merce & George & $\ldots$ \\
$\ldots$ & $\ldots$ & $\ldots$ & . & $\ldots$ & $\ldots$ & $\ldots$ \\
\hline
\end{tabular}

Table 1.1: Example of a partial event log for the scholarship process, grouped by case.

The information contained on the event logs represents an unbiased reflect of the real process as it is, being the perfect starting point for process mining techniques. 


\subsection{Process Mining}

Process mining is a relative young research discipline that sits between machine learning and data mining on the one hand, and process modeling and analysis on the other hand. The idea of process mining is to discover, monitor and improve real processes by extracting knowledge from event logs readily available in today's IT systems [4].

Event logs are unbiased footprints representing the process as it is. This contrasts with the process assumed by the process owners, a perception possibly biased by their understanding of how the process is executed. Event logs are the starting point of all process mining techniques, that use them to discover, verify or extend models for the process. In some cases these models are used to gain insight, document, or analyze the process, for example to simulate future scenarios. In other cases, the model is used directly to support the process, for example as part of some workflow management system. But in any case, a model close to the reality is required to obtain good results. Figure 1.1 shows an overview of how process mining is structured.

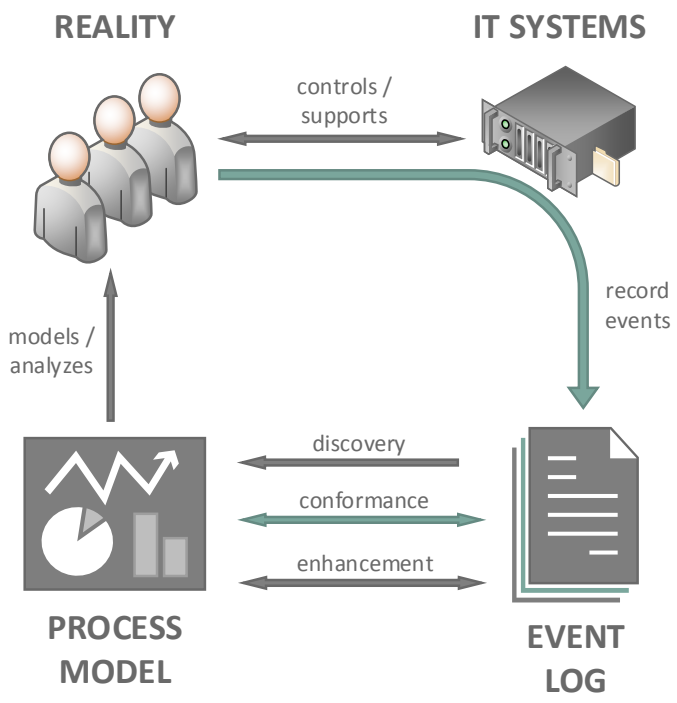

Figure 1.1: Overview of process mining and its three types of techniques: discovery, conformance and enhancement [4].

Figure 1.2 shows a possible workflow model discovered from the scholarship process event $\log$ of Table 1.1. For simplicity purposes, the model is defined using an informal but intuitive notation, instead of wide-spread process formalisms such as BPMN [69], Petri nets [68], or 
YAWL [9]. In this informal notation $A N D$ diamonds model concurrent activities and $X O R$ diamonds represent mutually exclusive activities, similar to the gateways of BPMN.

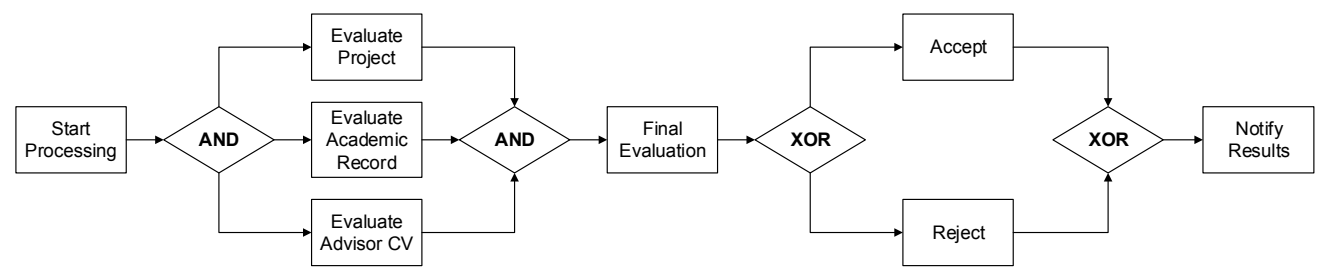

Figure 1.2: Informal process model of the scholarship process.

Process mining techniques can be grouped into three classes depending on their purpose: discovery, conformance, and enhancement.

Discovery: A discovery technique takes an event log as input and produces a model only considering the knowledge extracted from the log. For example, the model in Figure 1.2 could be an example of model obtained from logs of the scholarship process. Discovery is the oldest sub-discipline in process mining, and it is the one with more approaches proposed. The list of approaches is long, as long is the list of different techniques used to discover models. For instance, $\alpha$-algorithm [13] is based on detecting relations among activities of the log, Genetic Miner [58] uses evolutionary algorithms, or Genet [37] and ILP Miner [101] are based on the theory of regions. The model discovered is not restricted to the workflow perspective of the process. If the log contains additional information one can also discover resource-related models, for example capturing the interactions between the actors of the process [11].

Conformance: Unlike discovery, conformance considers both an event log and a model as inputs. The goal of conformance techniques is to check if the behavior modeled represents accurately the behavior observed in the log. Some examples of conformance techniques are $[79,15,62,67,97]$. Conformance can be used to verify if a process documentation is still updated, or to check if the model obtained using a discovery approach is a faithful representation of the process. Conformance checking is the main goal of this thesis and it is presented in more detail in Section 1.2.

Enhancement: Like conformance, enhancement also considers both an event log and a model as inputs. However, enhancement techniques aim to improve the a-priori model with information contained in the log. This enhancement could be done by means of repairing the model to better conform the observed behavior [44]. Another option is to extend the model with additional information, adding a new perspective to the process 
model. For example, detecting and including the data dependencies that affect the routing of a process execution [83].

\subsection{Conformance Checking and its Challenges}

Most of the research in process mining has mainly focused on discovery techniques, neglecting the importance of conformance. However, conformance techniques have become an essential part in the process life cycle. Regulations, certifications or simply communication purposes are making most organization document their processes. Process models are also required in process-aware information systems such as workflow management systems, or for analyzing and simulating future scenarios. However, models not representing adequately the reality could lead to wrong conclusions. Therefore, conformance techniques are necessary to detect inadequate models, or models that have become obsolete due the continuously changing nature of the processes. Conformance is also necessary to compare several models for the same process and determine the best option, for example among models obtained with different discovery algorithms.

\subsubsection{Dimensions of Conformance Checking}

By measuring the conformance between an event log and a process model one is concerned about quantifying if a given model is valid description of the reality. A first naive approach could be to consider that a model and a log conform each other simply if the model captures all the behavior observed in the log. In other words, a perfect conformance would require that all the traces in the log fit in the model. However, a more accurate study shows that, although important, this condition alone is not sufficient. For example, let us consider the model in Figure 1.3 for the scholarship example. The informal semantics behind this model (similar to Petri nets), know as a flower model, is that it captures a possible sequence of the activities, in any order and for any length. Therefore, any possible log involving the same activities fit this model. However, as one can see, this model provides absolutely no insight into the process or how the activities are executed.

In $[80,79]$ the multidimensional nature of the conformance is studied, and the authors propose four dimensions - fitness, precision, generalization and simplicity - to fully capture the notion of how good a given model is with respect to the reality.

Fitness As it has been already mentioned, this dimension indicates how much of the observed behavior is captured - fits - the process model. For example, the trace 〈Start Processing, Evaluate Academic Record, Evaluate Project, Evaluate Advisor CV, Final Evaluation, Accept, Notify Results $\rangle$ with case id 1 in Table 1.1 perfectly fits the model in Figure 1.2. However, the trace Start Processing, Evaluate Academic Record, Evaluate Project, Final Evaluation, Reject, Notify Results with case id 3 does not fit the model because the advisor CV is never checked, denoting that the application of 


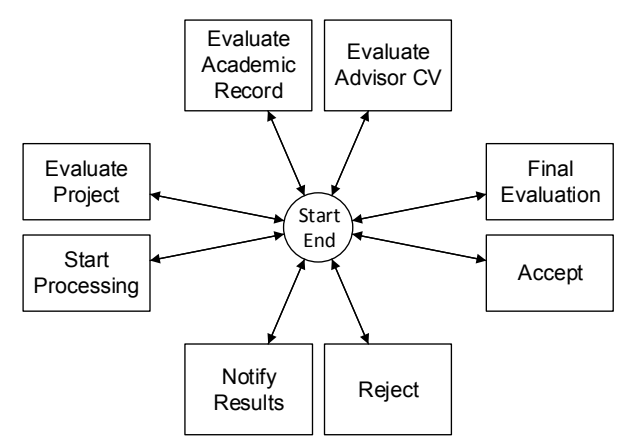

Figure 1.3: Informal flower process model of the scholarship process, modeling any possible sequence of the activities.

the student is rejected without proper evaluation. On the other hand, both traces fit the flower model of Figure 1.3. Part III of this thesis is devoted to analyze the fitness dimension in a decomposed way, and consequently a more formal presentation of the fitness dimension is included in these sections.

Precision This dimension addresses overly general models: precision penalizes a process model for allowing behavior that is unlikely given the observed behavior in the event log. For example, in the log of Table 1.1 we observe that, although the three documents could be evaluated concurrently, the university employees always evaluate first the academic record. That way, if the student is clearly not suitable for the grant (e.g., the grade does not reaches the minimum necessary), the advisor and project evaluation is done superficially. Consequently, the model shown in Figure 1.4 is a more precise representation of the reality than Figure 1.2. The flower model in Figure 1.3 is the perfect example of completely imprecise model. Part II of this thesis is devoted to the precision dimension, and consequently a more formal presentation of the precision is included in these sections.

Generalization This dimension addresses overfitting models: a good model must be able to generalize and reproduce possible future behavior, instead of capturing simply each trace of the observed log. For example, Figure 1.5 shows a model that only captures one possible order for the evaluation of the documents and that results necessarily in the acceptance of the application. This model perfectly captures the first trace in the Table 1.1, but it is unable to generalize for any other possible process execution.

Simplicity This dimension penalizes models that are unnecessarily complex: following 


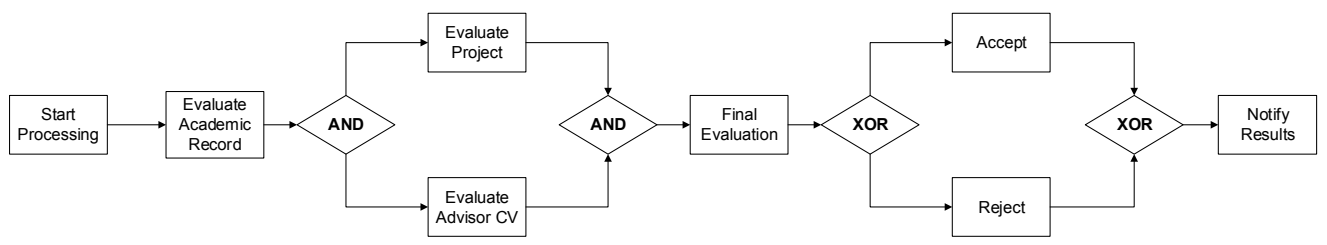

Figure 1.4: More precise model for the scholarship process.

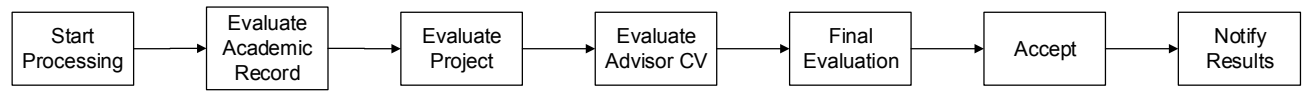

Figure 1.5: Model that overfitting the first trace of the scholarship log, and does not generalize for possible future behavior.

the Occam's Razor principle, models that explain the behavior observed in the log in a simple way are preferred to those that use redundant components. Figure 1.6 is an example where the unjustified use of duplicate activities - i.e., an activity of the process is represented in different parts of the model - complicates unnecessary a model for the scholarship process.

\subsubsection{Replay-based and Align-based Conformance Checking}

In early works on conformance, most of the proposed approaches were based on replaying the log on the model to detect discrepancies. Some replay-based approaches simply stop at the point where the model is not able to reproduce the trace anymore. Other approaches perform the replay in a non-blocking way, regardless whether the path of the model is followed or not, like [80]. More sophisticate approaches, like [98], include also a look ahead function to determine the most promising path. Recently, another family of approaches has appeared, where the conformance checking is done in a global manner, by means of aligning both the modeled behavior and the behavior observed in the log. Examples of conformance approaches based on alignments are $[15,54]$. These approaches handle conformance in a global way, but they are computationally more expensive compared with replay-based approaches. In the first part of this thesis, both replay-based and align-based approaches are explored to check precision. In the second part, a decomposed technique is proposed to alleviate the conformance diagnosis computation time, specially for those analysis based on alignments due their expensive cost. 


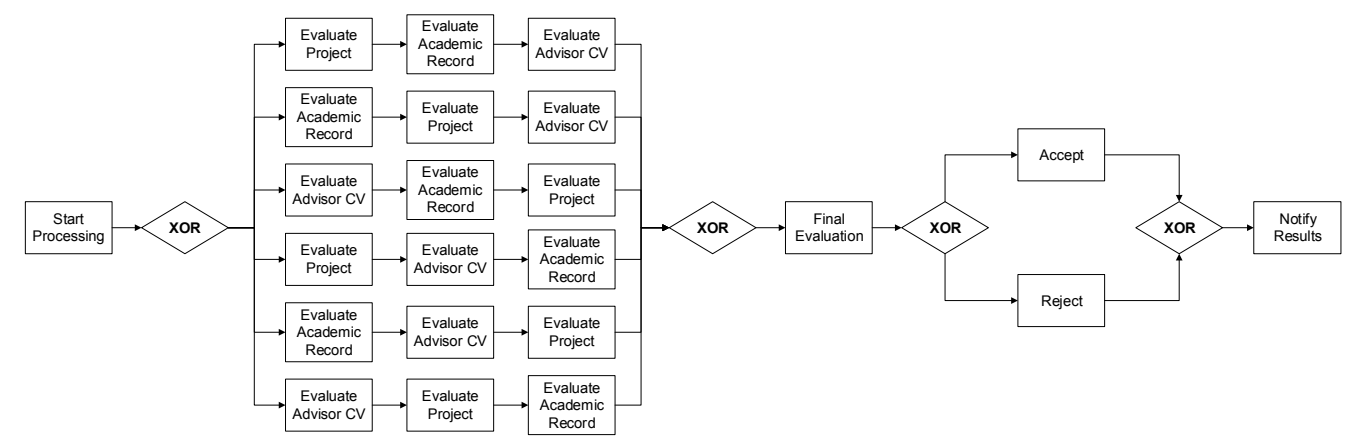

Figure 1.6: Unnecessary complex model for the scholarship process.

\subsubsection{Challenges of Conformance Checking}

Conformance checking must confront a set of challenges in order to be applied successfully. In particular, we identify five challenges: four-dimensional conformance, big data and real time, noise, incompleteness, unfitness, and indeterminism, conformance diagnosis and modeling notations. This thesis addresses all these the challenges. In recent years other authors have also proposed approaches in that direction.

Challenge 1 - Four-Dimensional Conformance. Since the multidimensional nature of the conformance - fitness, precision, generalization and simplicity - was first stated in [82] and later refined in [84, 80, 79], the relation between the four dimensions and the adequacy of the results has become more and more clear. Works like [33] illustrate the need of metrics for all the dimensions in order to discover good models. However, most of the approaches proposed in conformance, specially on the early days, are focused exclusively on fitness. Conformance checking must provide also metrics for other dimensions such as precision, generalization, and simplicity.

Challenge 2 - Big Data and Real Time. The amount of information recorded by the information systems periodically grows exponentially. Event logs become more detailed, complete and large, and with them also the process models. Conformance techniques must evolve accordingly in order to handle this exponential growth, specially those based on the global aligning of behaviors. Moreover, the fast implantation of online and monitoring paradigms in nowadays systems is requiring faster and more fine-grained conformance approaches.

Challenge 3 - Noise, Incompleteness, Unfitness, Indeterminism. Most of the problems found in process mining have to deal with this four concepts: noise, incompleteness, unfitness, and indeterminism. Noise in event logs can appear by traces incorrectly 
recorded (for instance, due to temporary system failure), or traces reflecting exceptional situations not representative for the typical behavior of the process. Noise is a wellknown problem in discovery approaches [4], and therefore, conformance approaches proposed should also be noise-aware too. On the other hand, assuming a complete $\log$ is an unrealistic assumption in most of the cases. The number of traces necessary for a complete log grows exponentially when the number of concurrent actions in the model is increased. Moreover, some concurrent actions may look sequentially in the $\log$ because performing one action is always much faster than the other. Conformance techniques must include mechanisms to aid the process analyst on deciding whether the problems are real conformance anomalies or result of the incompleteness of the $\log$. Unfitness - i.e., situations where the behavior observed in the $\log$ cannot be reproduced by the model - is a conformance dimension itself, but it may influence other dimensions: if the model cannot reproduce the observed behavior, it cannot determine in what state was the system in that moment. Conformance approaches should try to abstract from how the alignment between observed and modeled behavior is done. This include also the indeterministic situations produced when a trace in the log can be mapped to several sequences in the model.

Challenge 4 - Conformance Diagnosis. The importance of indicating the location of the problems for a proper conformance diagnosis was already emphasized in the seminal work [79]. However, the diagnosis mechanisms cannot be limited to simply locate the possible conformance errors, but they must go a step further: they must provide aiding mechanisms to the analyst to fully understand the root causes of the problems. For example, making it possible to dynamically inspect the conformance results at different levels of abstraction, or to group mismatches with a similar root cause. Diagnosis tools are specially useful for large models or models with a high degree of complexity, where the causes of the problems are difficult to grasp.

Challenge 5 - Modeling Notations. Most of the approaches presented in conformance so far focus exclusively on the control-flow perspective of the process - i.e., the order of the activities - and to one specific workflow modeling notation, Petri nets [68]. Conformance techniques must include other modeling notations, and other perspectives. In addition, there must appear new approaches to check the conformance of multi-perspective models - models capturing more than one perspective - like for example [54], where integer linear programming techniques are used to validate both the control-flow and the data perspectives of a model.

\subsection{Contributions of the Thesis}

Process mining, and more specifically conformance checking and conformance diagnosis, are the main goals of the contributions proposed in this thesis. These contributions can 
be grouped in two categories: those that aim on analyzing precision, and those that use decomposition to diagnose conformance problems in a decomposed way. Table 1.2 summarizes the contributions of this thesis. The table includes a description of the contribution, the category it belongs to, the chapter where they are presented, the conformance challenge they mainly address (cf. Section 1.2), and the publications where they were proposed. The remainder of the section enumerates the contributions of this thesis.

\begin{tabular}{|c|c|c|c|c|}
\hline & Contribution & Chapter & Challenge & Publication \\
\hline \multirow[t]{5}{*}{ Prec } & $\begin{array}{l}\text { Approach to quantify and analyze the } \\
\text { precision between a log and a model } \\
\text { based on escaping arcs. }\end{array}$ & 3 & 1,2 & {$[62]$} \\
\hline & $\begin{array}{l}\text { Robustness and confidence interval for } \\
\text { precision based on escaping arcs. }\end{array}$ & 4 & $1,2,3$ & {$[64][63]$} \\
\hline & $\begin{array}{l}\text { Severity assessment of the imprecision } \\
\text { point detected. }\end{array}$ & 4 & 1,4 & {$[64][63]$} \\
\hline & $\begin{array}{l}\text { Precision checking based on aligning ob- } \\
\text { served and modeled behavior. }\end{array}$ & 5 & 1,3 & {$[18]$} \\
\hline & $\begin{array}{l}\text { Abstraction and directionality in preci- } \\
\text { sion based on alignments. }\end{array}$ & 5 & 1,3 & {$[19]$} \\
\hline \multirow[t]{4}{*}{ Decomp } & $\begin{array}{l}\text { Decomposed conformance checking } \\
\text { based on SESE components. }\end{array}$ & 6 & 2,4 & {$[65][67]$} \\
\hline & $\begin{array}{l}\text { Hierarchical and topological decomposi- } \\
\text { tion based on SESE components for con- } \\
\text { formance diagnosis. }\end{array}$ & 7 & 4 & {$[66][67]$} \\
\hline & $\begin{array}{l}\text { Decomposed conformance checking for } \\
\text { multi-perspective models. }\end{array}$ & 8 & 5 & {$[55]$} \\
\hline & $\begin{array}{l}\text { Decomposed Conformance Checking for } \\
\text { Real-Time scenarios. }\end{array}$ & 9 & 2 & {$[29]$} \\
\hline
\end{tabular}

Table 1.2: Chart summarizing the contributions of this thesis.

\subsubsection{Precision Checking}

- A novel approach to measure precision in conformance checking, based on detecting and assessing escaping arcs. The escaping arcs identify where the modeled behavior allows 
more than the behavior observed in the log. Among its main features, the proposed metric is considerable less time-consuming than previous ones such as [80].

- Extension of the precision checking to be more robust against noisy or infrequent traces of the log. It complements the approach with an interval of confidence over the metric provided. A wide interval denotes a low confidence on the results, whereas a narrow interval represents the unlikelihood of a future variation of the metric.

- Severity assessment of the imprecise points. All points of impreciseness are evaluated by a multi-factor metric, denoting the priority of each one to be solved.

- Using the Alignment of observed and modeled behavior to detect precision problems. The global nature of the aligning techniques solves the possible problems of unreplayable logs - the model cannot mimic the "moves" of the log -, problems present on replay-based conformance approaches.

- Extension for the precision checking to include different levels of abstraction. The extension includes the possibility to abstract from the order of the activities - two traces with the same activities in different order represent the same observed behavior - and the abstraction of the direction of the process - giving more importance to the start or the end of the process.

\subsubsection{Decomposed Conformance}

- A novel approach to decompose conformance checking based on partitioning the model into Single-Entry Single-Exit (SESE) components. Informally, a SESE component is a part of the model with only one entry and only one exit nodes, denoting a subprocess with simple interface with respect to the main modeled process. The approach correctness is formally proven. In practice, this decomposition technique alleviates significantly the computation time of the current conformance techniques, specially those based on alignments.

- A topological representation of the SESE partitioning as starting point for detecting parts or sets of parts with conformance anomalies. Hierarchical SESE decomposition to analyze and diagnose possible conformance problems at different levels of abstractions.

- Formalization of decomposed conformance for multi-perspective models. In particular, Petri nets with data containing both control-flow and data perspectives. Definition of several decomposition strategies for different conformance scenarios.

- Application of decomposition for real-time scenarios, where the model to be monitored is large or complex, and events are provided in a streaming context. The decomposition provides mechanisms to aid on conformance diagnosis and alleviate the computation times critical in real-time systems. 


\subsection{Structure of the Thesis}

The thesis is structured in four main parts.

- Part I provides an introduction to the thesis. It consists of an introduction in Chapter 1, and the necessary preliminaries to fully understand the thesis in Chapter 2.

- Part II presents the techniques proposed for precision checking. Chapter 3 introduces an approach to measure and analyze the precision between an event log and a process model, and Chapter 4 incorporates robustness, confidence and severity assessment to the approach. Finally, Chapter 5 discusses the use of alignments between observed and model behaviors for precision checking purposes.

- Part III proposes the use of decomposition to check conformance. Chapter 6 introduces the SESE decomposition of models for a faster and meaningful checking. Chapter 7 discusses the use of hierarchical decomposition and topological representations as tools for conformance diagnosis. Chapter 8 describes the decomposition for multi-perspective models, while Chapter 9 proposes the application of decomposition techniques to eventbased real-time scenarios.

- Part IV and Chapter 10 concludes the thesis, summarizing the main results and discussing possible extensions of the work presented.

\subsection{Publications and Thesis Impact}

The materials reported in this thesis are partially published in international journals in the areas of business process management and information systems: [67] in Information Systems, [19] in Information Systems and e-Business Management, and [63] in the special issue on business process management of the International Journal of Innovative Computing, Information and Control.

In addition, materials of this thesis were presented in international conferences of different fields: [62] and [65] in the International Conference on Business Process Management (BPM), [64] in the IEEE Symposium on Computational Intelligence and Data Mining (CIDM), [66] in Application and Theory of Petri Nets and Concurrency, and [18] in the workshop of Business Process Intelligence (BPI). Finally, [29] and [55] were presented in the International Conference on Cooperative Information Systems (CoopIS).

The applicability of the approaches proposed is extensively used in the area, with more than 130 citations among all the publications, according to Google Scholar. The metrics for precision proposed in this thesis have been used by the community as a reference conformance measures to evaluate different discovery algorithms [96], to guide discovery techniques based on genetic algorithms [33], as part of the CoBeFra conformance benchmarking framework [28], or to train a recommender system for process discovery [78], among other applications. 


\section{Chapter 2}

\section{Preliminaries}

This chapter introduces the preliminaries used in the remainder of this thesis. Some basic notations are introduced in Section 2.1. Section 2.2 and Section 2.3 formalize the concepts of event logs and process models, the two necessary elements in any conformance checking approach. Finally, Section 2.4 presents several process model formalisms, including Petri nets (and extensions), used to illustrate most of the thesis. For the sake of comprehension, the concepts introduced in this section respects de facto notations used in most of works of the field, such as $[67,1,79,15]$.

\subsection{Basic Notations}

In this section we introduce the basic notations for sets, multisets, functions, sequences, and transition systems.

Definition 2.1 (Set). A set $A$ is a possible infinite collection of elements. The elements in the set are listed between braces, e.g., $A=\{a, b, c\}$. The empty set is represented by $\emptyset$. $|A|$ denotes the size of the set, e.g., $|A|=3 . \mathcal{P}(A)$ denotes the powerset of $A$, the set of all subsets of $A$, including the empty set and $A$ itself, e.g., $\mathcal{P}(A)=\{\emptyset,\{a\},\{b\},\{c\},\{a, b\},\{a, c\},\{b, c\}$, $\{a, b, c\}\}$.

Some operations are defined over sets. Let $A=\{a, b, c, d\}$ and $B=\{a, c, d, e\}$ be nonempty sets. The union of $A$ and $B$, denoted $A \cup B$, is the set containing all elements of either $A$ or $B$, e.g., $A \cup B=\{a, b, c, d, e\}$. The intersection of $A$ and $B$, denoted $A \cap B$, is the set containing elements in both $A$ and $B$, e.g., $A \cap B=\{a, c, d\}$. The difference between $A$ and $B$, denoted $A \backslash B$, is the set containing all elements of $A$ that are not in $B$, e.g., $A \backslash B=\{b\}$.

Multisets are used to describe event logs and to represent states of some processes models such as Petri nets. 
Definition 2.2 (Multiset). A multiset - also called $b a g-B$ over a set $A$ is a possible infinite collection of elements of $A$, where each element may appear more than once. The elements in the multiset are listed between square brackets, e.g., $B=[a, a, b]$, also denoted as $B=\left[a^{2}, b\right]$, where $A=\{a, b\} . B(a)$ denotes the number of times the element $a$ appears in the multiset $B$, e.g., $B(a)=2, B(b)=1$ and $B(c)=0$ for all $c \notin A$. Furthermore, a set $S \subseteq A$ can be viewed as a multiset where each element occurs once. The empty multiset is denoted as []. $\mathcal{B}(A)$ represents the set of all multisets over the set $A$.

The standard operations over sets can be extended to multisets. For example: the union $[a, a] \uplus[a, b]=[a, a, a, b]$, the difference $[a, a, b, c] \backslash[a, b, b]=[a, c]$, the size $|[a, a]|=2$, etc.

Sequences are used to represent traces in the log, or states of the observed behavior and modeled behavior.

Definition 2.3 (Sequence). A sequence $\sigma=\left\langle a_{1}, a_{2}, \ldots, a_{n}\right\rangle \in A^{*}$ over a set $A$ is an ordered list of elements $a_{i} \in A$ of size $n$. The empty sequence is denoted as \langle\rangle . The concatenation of sequences is denoted as $\sigma_{1} \cdot \sigma_{2}$.

Functions are constantly used in the thesis to represent different meanings, such as the mapping between model tasks and observable activities.

Definition 2.4 (Function). Let $A$ and $B$ be non-empty sets. A function $f$ from $A$ to $B$, denoted $f: A \rightarrow B$ is a relation from $A$ to $B$, where every element of $A$ is associated to an element of $B$. Given a function $f: A \rightarrow B, \operatorname{dom}(f)$ and $r n g(f)$ denote the domain and the range of $f$. A partial function, denoted $f: A \nrightarrow B$, is a function that may be undefined for some of the elements in $A$.

In particular, a function can be applied to a sequence, applying it only to the elements in the domain of the function, i.e. if $\operatorname{dom}(f)=\{x, y\}$, then $f(\langle y, z, y\rangle)=\langle f(y), f(y)\rangle$.

Definition 2.5 (Function to Sequences). Let $f \in X \nrightarrow \rightarrow Y$ be a partial function. $f$ can be applied to sequences of $X$ using the following recursive definition (1) $f(\langle\rangle)=\langle\rangle$ and (2) for $\sigma \in X^{*}$ and $x \in X$ :

$$
f(\langle x\rangle \cdot \sigma)= \begin{cases}f(\sigma) & \text { if } x \notin \operatorname{dom}(f) \\ \langle f(x)\rangle \cdot f(\sigma) & \text { if } x \in \operatorname{dom}(f)\end{cases}
$$

A specially useful function is the projection.

Definition 2.6 (Projection). Let $X$ be a set and $Q \subseteq X$ one of its subsets. $\uparrow_{Q} \in X^{*} \rightarrow Q^{*}$ is a projection function and is defined recursively: (1) $\left.\langle\rangle\right|_{Q}=\langle\rangle$ and (2) for $\sigma \in X^{*}$ and $x \in X$ :

$$
\left.(\langle x\rangle \cdot \sigma)\right|_{Q}= \begin{cases}\sigma \uparrow_{Q} & \text { if } x \notin Q \\ \langle x\rangle \cdot \sigma \uparrow_{Q} & \text { if } x \in Q\end{cases}
$$


For example, $\left.\langle y, z, y\rangle\right|_{\{x, y\}}=\langle y, y\rangle$.

Transition systems are used to represent the observed and the modeled behavior. Moreover, in other to abstract from any specific process model notation, transition systems are used to represent the semantics of a generic process model.

Definition 2.7 (Transition System). A transition system with initial states $S_{0}$ and final states $S_{F}$ is a tuple $T S=\left\{S, \Sigma, \nearrow, S_{I}, S_{F}\right\}$ where $S$ is a set of states, $\Sigma$ is a set of action labels, $S_{I} \subseteq S$ is a set of initial states, $S_{F} \subseteq S$ is a set of final states, and $\nearrow \subset S \times \Sigma \times S$ is a transition relation.

A more general - and more commonly used - definition of transition systems does not include the set of final states. When no set of final states is specified, it is assumed all states are final states, i.e., $S_{F}=S$. Figure 2.1 shows an example of transition system with states $S=\left\{s_{1}, s_{2}, s_{3}, s_{4}\right\}$ and labels $\Sigma=\{a, b, c\}$, being $s_{1}$ its initial state. The transition system denotes that, for example, there is a transition from the state $s_{1}$ to the state $s_{2}$.

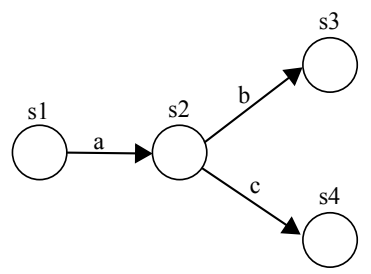

Figure 2.1: Example of TS with $S=\left\{s_{1}, s_{2}, s_{3}, s_{4}\right\}, \Sigma=\{a, b, c\}$, and $S_{I}=\left\{s_{1}\right\}$.

\subsection{Event Logs}

Event logs are the footprints left in the system by the execution of processes. They are the main object any process mining technique works with. Let us consider a variant of the scholarship process presented in Chapter 1. In this variant both Start Processing and Notify Results activities are substituted by a single activity Set Checkpoint executed at the start and end of the process. Moreover, the Final Evaluation action is done always outside the system, and there is no recording of it in the log. Table 2.1 shows a possible partial event log of the process. The log contains two complete process executions or cases: Alex and Dolores application. Each row corresponds with one event in the log, and the events are ordered chronologically. Therefore, an event $\log$ is a sequence of events. Each event is associated with a set of attributes. The list of the most common attributes in event logs for the practice of process mining analysis are:

- case - process instance id of the event.

- activity - name of the action performed in the event. 


\begin{tabular}{|c|c|c|c|c|c|}
\hline Event & Case & Timestamp & Activity & Employee & Student \\
\hline 1 & 1 & 01-01-2014 10:00 & (a) Set Checkpoint & Merce & Alex \\
\hline 2 & 1 & 01-01-2014 11:30 & (c) Evaluate Academic Record & Fernando & Alex \\
\hline 3 & 2 & 01-01-2014 12:00 & (a) Set Checkpoint & Merce & Dolores \\
\hline 4 & 1 & 01-01-2014 13:30 & (b) Evaluate Project & Fernando & Alex \\
\hline 5 & 2 & 01-01-2014 14:00 & (c) Evaluate Academic Record & Maria & Dolores \\
\hline 6 & 2 & 01-01-2014 15:00 & (d) Evaluate Advisor CV & Maria & Dolores \\
\hline 7 & 2 & 01-01-2014 16:00 & (b) Evaluate Project & Fernando & Dolores \\
\hline 8 & 1 & 01-01-2014 17:00 & (d) Evaluate Advisor CV & Fernando & Alex \\
\hline 10 & 1 & 02-01-2014 11:00 & (e) Accept & Ana & Alex \\
\hline 11 & 1 & 02-01-2014 12:00 & (a) Set Checkpoint & Merce & Alex \\
\hline 12 & 2 & 02-01-2014 13:00 & (f) Reject & Ana & Dolores \\
\hline 13 & 2 & 02-01-2014 16:00 & (a) Set Checkpoint & Merce & Dolores \\
\hline
\end{tabular}

Table 2.1: Example of event log for the scholarship process variant, ordered chronologically.

- timestamp - moment of the event execution, establishing an order between the events.

- resource - name of the resource initiating the event.

- data - data attribute related to the event.

For example, the event 10 is part of the case 1, corresponding with the scholarship application of Alex, and it reflects an Accept activity performed by Ana at $11: 00$ on the date $02-01-$ 2014. For the sake of clarity activities have associated a lowercase letter, e.g., $e=$ Accept.

Different attributes are required to derive different type of models. For example, the resource attribute is necessary for discovering a social interactions between actors of the process. In this thesis we focus mainly on the control-flow perspective of the processes (except on Chapter 8 were we also focus on the data perspective). For those cases, the activity, the case and an order between events is necessary, and therefore, the definition of event logs is simplified: the event log is composed by traces, where each trace corresponds to a case, and only the activity is considered for each event.

Definition 2.8 (Trace, Event $\log$ ). Let $A \in \mathcal{U}_{A}$ be a set of activities in some universe of activities $\mathcal{U}_{A}$. A trace $\sigma \in A^{*}$ is a sequence of activities. An event $\log$ is a multiset of traces, i.e., $L \in \mathcal{B}\left(A^{*}\right)$.

For example, the event log in Table 2.1 is represented as $[\langle a, c, b, d, e, a\rangle,\langle a, c, d, b, f, a\rangle]$, containing information about 12 events and 2 cases, where each case follows a different trace.

Although they are called event logs, event information is rarely recorded in logs files as Apache logs or error logs are, but stored in some internal database. However, recently a new XML-based standard for event logs has been presented: eXtensive Event Stream (XES) [43]. 
The purpose of XES is not only the storage of event logs, but to provide a standard format for the interchange of event log data between tools and application domains.

\subsection{Process Models}

Process models are the second element necessary in any conformance checking approach. A process model captures the behavior to compare with respect to the behavior observed in the log. Different models are used to capture different perspective of the process. In this thesis we mainly focus on the control-flow perspective of the processes, and therefore the models are used to capture the ordering between the actions. For the sake of generality, whenever it is possible, we abstract from any specific process modeling notation defining a generic process model. In Section 2.4 we present several concrete process modeling languages.

A generic process model semantics can be abstracted using a basic transition systems as a placeholder for more advanced modeling languages, such as Petri nets, UML, BPMN, EPCs, etc.

Definition 2.9 (Process Model). A process model semantic is abstracted as a transition system $T S=\left\{S, T, \nearrow, S_{I}, S_{F}\right\}$ over a set of model tasks $T$ with states $S$, initial states $S_{I} \subseteq S$, final states $S_{F} \subseteq S$, and transitions $\nearrow \subset S \times T \times S$. When no set of final states is specified, all states are assumed to be final states.

A task $t \in T$ is enabled to be executed in the state $s$ of the model $M$, denoted as $(M, s)[t\rangle$, if there is a transition with task $t$ in the state $s$, i.e., $\left(s, t, s^{\prime}\right) \in \nearrow .(M, s)[t\rangle\left(M, s^{\prime}\right)$ denotes that $t$ is enabled in $s$ and executing $t$ results in the state $s^{\prime}$. Let $\sigma=\left\langle t_{1}, t_{2}, \ldots, t_{n}\right\rangle \in T^{*}$ be a sequence of model tasks. $(M, s)[\sigma\rangle\left(M, s^{\prime}\right)$ denotes that there is a set of states $s_{0}, s_{1}, \ldots, s_{n}$ such that $s_{0}=s, s_{n}=s^{\prime}$ and $\left(M, s_{i}\right)\left[t_{i+1}\right\rangle\left(M, s_{i+1}\right)$ for $0 \leq i<n$.

Definition 2.10 (Complete Task Sequences). The set of complete tasks sequences $\phi_{t}$ of process model $M$ is the set of all possible sequences of tasks executed from an initial state reaching a final state, i.e., $\phi_{t}(M)=\left[\sigma \mid\left(M, s_{I}\right)[\sigma\rangle\left(M, s_{F}\right)\right\}$ for all $s_{I} \in S_{I}$ and $s_{F} \in S_{F}$.

Figure 2.2 shows a process model $M$ capturing the order of the tasks $T=t_{1} \ldots t_{8}$ using the informal semantics of Chapter 1, i.e., $A N D$ models concurrent tasks and $X O R$ mutually exclusive tasks. The set of complete tasks sequences of this model is: 


$$
\begin{aligned}
& \left\langle t_{1}, t_{2}, t_{3}, t_{4}, t_{5}, t_{6}, t_{8}\right\rangle \\
& \left\langle t_{1}, t_{2}, t_{3}, t_{4}, t_{5}, t_{7}, t_{8}\right\rangle \\
& \left\langle t_{1}, t_{2}, t_{4}, t_{3}, t_{5}, t_{6}, t_{8}\right\rangle \\
& \left\langle t_{1}, t_{2}, t_{4}, t_{3}, t_{5}, t_{7}, t_{8}\right\rangle \\
& \left\langle t_{1}, t_{3}, t_{2}, t_{4}, t_{5}, t_{6}, t_{8}\right\rangle \\
\phi_{t}(M)= & \left\langle t_{1}, t_{3}, t_{2}, t_{4}, t_{5}, t_{7}, t_{8}\right\rangle \\
& \left\langle t_{1}, t_{3}, t_{4}, t_{2}, t_{5}, t_{6}, t_{8}\right\rangle \\
& \left\langle t_{1}, t_{3}, t_{4}, t_{2}, t_{5}, t_{7}, t_{8}\right\rangle \\
& \left\langle t_{1}, t_{4}, t_{2}, t_{3}, t_{5}, t_{6}, t_{8}\right\rangle \\
& \left\langle t_{1}, t_{4}, t_{2}, t_{3}, t_{5}, t_{7}, t_{8}\right\rangle \\
& \left\langle t_{1}, t_{4}, t_{3}, t_{2}, t_{5}, t_{6}, t_{8}\right\rangle \\
& \left\langle t_{1}, t_{4}, t_{3}, t_{2}, t_{5}, t_{7}, t_{8}\right\rangle
\end{aligned}
$$

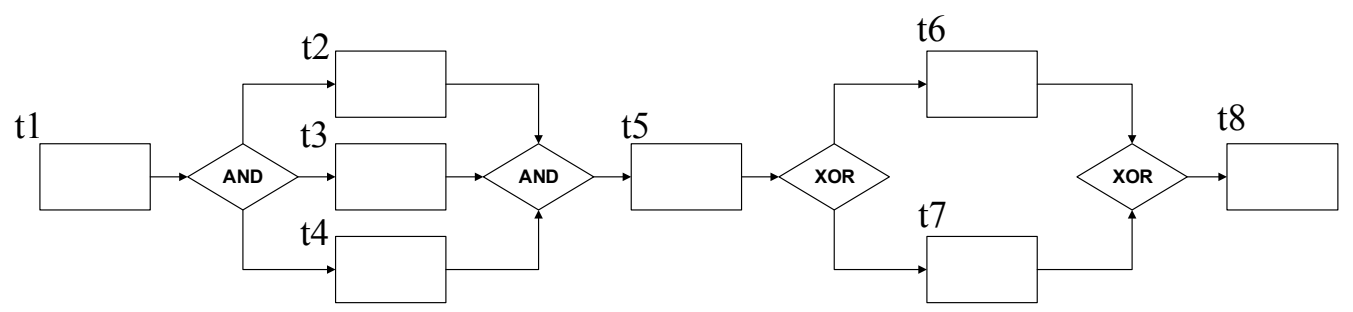

Figure 2.2: Informal process model for tasks $t_{1} \ldots t_{8}$.

In a labeled process model (or observable process model) the tasks represent activities in a real process that are potentially observable, i.e., they may cause events in a log. This potential observability is captured by the labeling function, which relates observable tasks in the model to activities of the process.

Definition 2.11 (Labeled Process Model). A labeled process model (or simply a process model) is a tuple $M=(T S, l)$, where the transition system $T S=\left\{S, T, \nearrow, S_{I}, S_{F}\right\}$ represents the semantics of the model, and $l \in T \nrightarrow \mathcal{U}_{A}$ is a labeling function where $\mathcal{U}_{A}$ is some universe of activity labels.

The process models in this thesis are considered always labeled, unless stated otherwise. If a model task $t \notin \operatorname{dom}(l)$, then it is called invisible (or also known as silent or unobservable). An occurrence of a visible task $t \in \operatorname{dom}(l)$ corresponds to observable activity $l(t)$. Two or more tasks associated with the same activity are called duplicate. For instance, Figure 2.3 show a labeled process model for the scholarship variant process. The task $t_{5}$ is invisible, i.e., this action is not observed nor recorded in the log. Tasks $t_{1}$ and $t_{8}$ are duplicate tasks corresponding with the same activity (a)Set Checkpoint. 


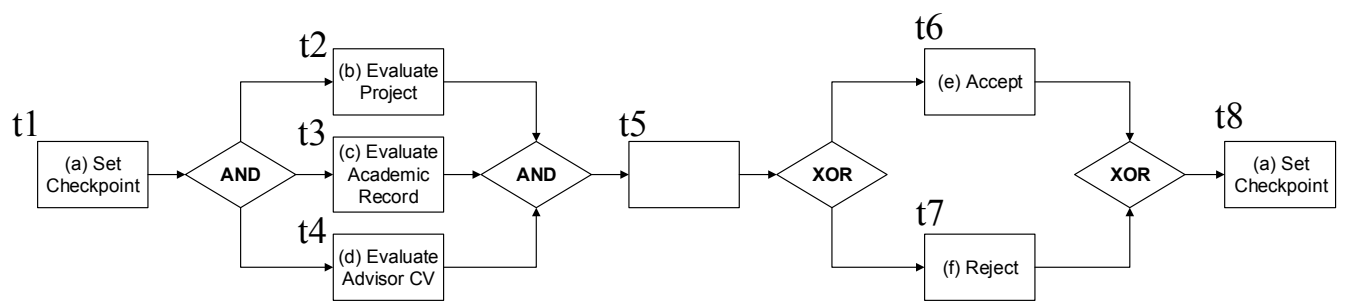

Figure 2.3: Informal labeled process model for scholarship variant process.

Definition 2.12 (Process Model notations). Let $M=(T S, l)$ be a process model with $T S=\left\{S, T, \nearrow, S_{I}, S_{F}\right\}$.

- $T_{v}(M)=\operatorname{dom}(l)$ is the set of visible tasks in $M$.

- $A_{v}(M)=r n g(l)$ is the set of corresponding observable activities in $M$.

- $T_{v}^{u}(M)=\left\{t \in T_{v}(M) \mid \forall_{t^{\prime} \in T_{v}(M)} l(t)=l\left(t^{\prime}\right) \Rightarrow t=t^{\prime}\right\}$ is the set of unique visible tasks in $M$, i.e., there are no other tasks with the same associated to the same activity.

- $A_{v}^{u}(M)=\left\{l(t) \mid t \in T_{v}^{u}(M)\right\}$ is the set of corresponding unique observable activities in model $M$.

In the example model $M$ of Figure 2.3 the set of visible tasks is $T_{v}(M)=\left\{t_{1}, t_{2}, t_{3}, t_{4}, t_{6}, t_{7}, t_{8}\right\}$ (all except $t_{5}$ ), and the set of unique visible tasks is $T_{v}^{u}(M)=\left\{t_{2}, t_{3}, t_{4}, t_{6}, t_{7}\right\}$. The set of observable activities is $A_{v}(M)=\{a, b, c, d, e, f\}$, while the set of unique observable activities is only $A_{v}^{u}(M)=\{b, c, d, e, f\}$.

Similar to the set of complete task sequences, a labeled process model contains its corresponding set of complete activity sequences, i.e., sequences of tasks starting from an initial to a final state projected onto the set of observable activities.

Definition 2.13 (Complete Activity Sequences). Let $M$ be a process model with $T$ tasks and labeling function $l$. A sequence $\sigma_{v}=\left\langle a_{1}, a_{2}, \ldots, a_{n}\right\rangle \in \mathcal{U}_{A} *$ is a complete activity sequence of the model $M$, denoted as $\left(M, s_{I}\right)\left[\sigma_{v} \triangleright\left(M, s_{F}\right)\right.$ if and only if there is a complete tasks sequence $\sigma \in T^{*}$ in $M$ such that $\left(M, s_{I}\right)[\sigma\rangle\left(M, s_{F}\right)$ and $l(\sigma)=\sigma_{v}$. The set of complete activity sequences of the model $M$ is denoted $\phi(M)$.

The set of complete activity sequences of the model in Figure 2.3 is: 


$$
\begin{aligned}
& \langle a, b, c, d, e, a\rangle \\
& \langle a, b, c, d, f, a\rangle \\
& \langle a, b, d, c, e, a\rangle \\
& \langle a, b, d, c, f, a\rangle \\
& \langle a, c, b, d, e, a\rangle \\
\phi(M)= & \langle a, c, b, d, f, a\rangle \\
& \langle a, c, d, b, e, a\rangle \\
& \langle a, c, d, b, f, a\rangle \\
& \langle a, d, b, c, e, a\rangle \\
& \langle a, d, b, c, f, a\rangle \\
& \langle a, d, c, b, e, a\rangle \\
& \langle a, d, c, b, f, a\rangle
\end{aligned}
$$

\subsection{Process Modeling Formalisms}

There are a wide variety of process modeling formalism that match the generic process model definition of previous section. In this section we present some of these formalisms. In particular we focus on Petri nets and its extensions, the formal notation used to illustrate the process models examples of this thesis.

\subsubsection{Petri Nets}

Petri nets [68] are one of most frequently used process modeling notations in process mining. Its formal semantics, its mathematical foundation, and its inherent capacity to model concurrency in a succinct way, make Petri nets perfect to model the control-flow perspective of processes. In addition, Petri nets are supported by an intuitive graphical notation, and there exists a wide range of tools and libraries to operate with them.

Definition 2.14 (Petri Net). A Petri net is a tuple $P N=(P, T, F)$ with $P$ the set of places, $T$ the set of transitions, where $P \cap T=\emptyset$, and $F \subseteq(P \times T) \cup(T \times P)$ the flow relation. For a node $n$ (place or transition) of a Petri net, $\bullet n(n \bullet)$ is the predecessor (successor) set of $n$ in $A$, i.e., $\bullet n=\left\{n^{\prime} \mid\left(n^{\prime}, n\right) \in F\right\}$ and $n \bullet=\left\{n^{\prime} \mid\left(n, n^{\prime}\right) \in F\right\}$.

The set of transitions $T$ represent the set of tasks of generic process modeling definition. An example of Petri net is shown in Figure 2.4. The transitions are represented as square nodes, while the places are represented as circles.

The states of a Petri net are called markings. The formal semantics of Petri nets are defined by the firing rule, that states the effects of firing an enabled transition.

Definition 2.15 (Petri Net Semantics). Let $P N=(P, T, F)$ be a Petri net. A marking $M$ is a multiset of places, i.e., $M \in \mathcal{B}(P)$. A transition $t \in T$ is enabled in a marking $M$, denoted 

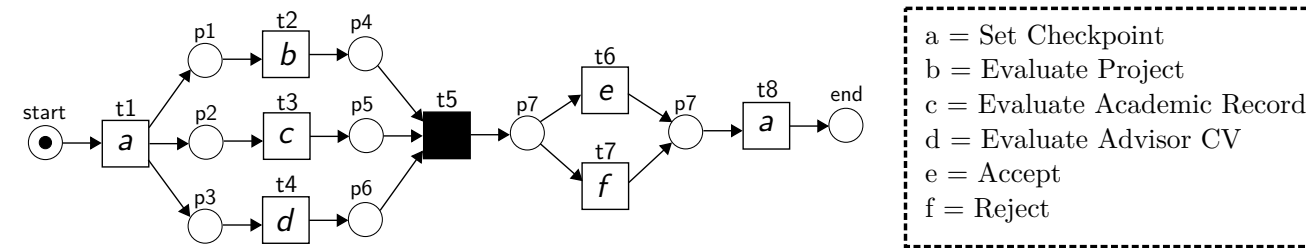

Figure 2.4: Petri net for scholarship variant process of Figure 2.3.

as $(P N, M)[t\rangle$, iff $\bullet t \leq$. Firing transition $t$ in $M$, denoted as $(P N, M)[t\rangle\left(P N, M^{\prime}\right)$, results in a new marking $M^{\prime}=M-\bullet t+t \bullet$, i.e., tokens are removed from $\bullet t$ and added to $t \bullet$.

A marking is graphically represented as black dots (called tokens) in places. For example, the marking represented in Figure 2.4 is $M=$ [start]. In that marking, only $t_{1}$ is enabled, and firing $t_{1},(P N, M)\left[t_{1}\right\rangle\left(P N, M^{\prime}\right)$, will result in the marking $M^{\prime}=\left[p_{1}, p_{2}, p_{3}\right]$. In $M^{\prime}$, $t_{2}, t_{3}, t_{4}$ are enabled simultaneously, and can be fired in any order.

Similar to tasks sequences, a transition sequence from a Petri net can also be defined. Notice that, for the sake of clarity, the same notation is preserved between generic process models and Petri nets, referring to tasks or transitions in each particular case.

Definition 2.16 (Transition Sequence). A transition sequence $\sigma=\left\langle t_{1}, t_{2}, \ldots, t_{n}\right\rangle \in T^{*}$ of Petri net $P N$, represented as $(P N, M)[\sigma\rangle\left(P N, M^{\prime}\right)$, denotes that there is a set of markings $M_{0}, M_{1}, \ldots, M_{n}$ such that $M_{0}=M, M_{n}=M^{\prime}$ and $\left(N, M_{i}\right)\left[t_{i+1}\right\rangle\left(N, M_{i+1}\right)$ for $0 \leq i<n$. A marking $M^{\prime}$ is reachable from $M$ if there exists a $\sigma$ such that $(P N, M)[\sigma\rangle\left(P N, M^{\prime}\right)$.

Similar to generic labeled process models, a Petri net can also be labeled, associating observable activities to the transitions of the model.

Definition 2.17 (Labeled Petri Net). A labeled Petri net $P N=(P, T, F, l)$ is a Petri net $(P, T, F)$ with labeling function $l \in T \nrightarrow \mathcal{U}_{A}$, where $\mathcal{U}_{A}$ is some universe of activity labels.

Figure 2.4 shows a labeled Petri net for scholarship variant process of Figure 2.3. Similar to the generic labeled process model, we can define the visible transitions $T_{v}(P N)$, observable activities $A_{v}(P N)$, unique visible transitions $T_{v}^{u}(P N)$, and unique observable activities $A_{v}^{u}(P N)$ of a Petri net $P N$. Typically, invisible transitions are represented as filled squares, e.g., $t_{5}$.

Definition 2.18 (Activity Sequence). A sequence $\sigma_{v}=\left\langle a_{1}, a_{2}, \ldots, a_{n}\right\rangle \in \mathcal{U}_{A} *$ is a activity sequence of the Petri net $P N$, denoted as $(P N, M)\left[\sigma_{v} \triangleright\left(P N, M^{\prime}\right)\right.$ if and only if there is a transition sequence $\sigma \in T^{*}$ in $P N$ such that $(P N, M)[\sigma\rangle\left(P N, M^{\prime}\right)$ and $l(\sigma)=\sigma_{v}$.

In the context of process mining and business processes, processes are usually considered to start in an initial state and to end in a well-defined end state. Petri nets considering a initial and a final marking are called system nets. 
Definition 2.19 (System Net). A system net is a triplet $S N=\left(P N, M_{I}, M_{F}\right)$ where $P N=$ $(P, T, F, l)$ is a labeled Petri net, $M_{I} \in \mathcal{B}(P)$ is the initial marking, and $M_{F} \in \mathcal{B}(P)$ is the final marking.

A system net $S N=(P N,[$ start $],[e n d])$ is a possible system net for the Petri net $P N$ in Figure 2.4. We define the set of complete transition sequences and the set of complete activity sequences as the sequence of transitions and activities from the initial marking to the final marking.

Definition 2.20 (Complete Transition Sequence, Complete Activity Sequence). Let $S N=$ $\left(P N, M_{I}, M_{F}\right)$ be a system net with $P N=(P, T, F, l)$. The set of complete transition sequences $\phi_{t}$ of $S N$ is the set of all possible sequences of transitions executed from the initial marking and reaching the final marking, i.e., $\phi_{t}(S N)=\left[\sigma \mid\left(P N, M_{I}\right)[\sigma\rangle\left(P N, M_{F}\right)\right\}$. The set of complete activity sequences of system net $S N$ is the set of all possible sequences of observable activities from the initial marking and reaching the final marking, i.e., $\phi(S N)=$ $\left[\sigma \mid\left(P N, M_{I}\right)\left[\sigma \triangleright\left(P N, M_{F}\right)\right\}\right.$.

\subsubsection{Workflow Nets}

Workflow nets [5, 7], commonly used for business and workflow processes, are a subclass of Petri nets with a well defined starting and ending place.

Definition 2.21 (Workflow Net). A workflow net $W N=(P, T, F, l$, start, end) is a particular type of Petri net where:

- start is a special source place with no incoming arcs, i.e., $\bullet$ start $=\emptyset$

- end is a special sink place, with no outgoing arcs, i.e., end $\bullet=\emptyset$

- every node of the net must be on some path from start to end

The Petri net in Figure 2.4 shown in the previous section is actually a workflow net. Workflow nets present a direct way to define system nets, with a single token in start as initial marking, and a single token in end as a final marking.

Definition 2.22 (System Net from Workflow net). A system net $S N=(W N,[$ start $]$, $[$ end $])$ from the workflow net $W N$ is the net where the initial marking is [start], and the final marking is $[e n d]$.

\subsubsection{Other Formalisms}

There is a wide range of other model formalisms to describe processes. Each formalism has its own advantages and disadvantages, and its own tools to support it. Some examples of such formalisms are $B P M N$ [69], YAWL [9], EPC [3], or $U M L$ [70], to enumerate some examples. 
Business Process Model and Notation (BPMN) [69] is a standard for business process modeling that provides a graphical notation for specifying business processes based on a flowcharting technique very similar to activity diagrams from Unified Modeling Language (UML) [70]. The objective of BPMN is to support business process management, for both technical users and business users, by providing a notation that is intuitive to business users, yet able to represent complex process semantics. BPMN is one of the most used notations in the industry. BPMN is composed of events (denoted as circles), activities (denoted as rounded squares) and gateways (denoted as diamonds), among other elements, and the connections between them. Figure 2.5 illustrates a model for the scholarship variant process using BPMN notation ${ }^{1}$.

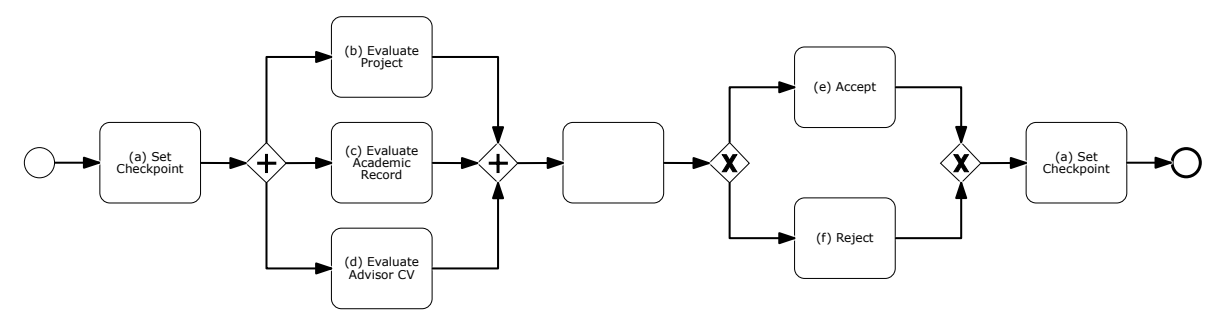

Figure 2.5: BPMN for scholarship variant process of Figure 2.3.

Another notation is YAWL [9]. The original drivers behind YAWL were to define a workflow language that would support all (or most) of the typical workflow patterns [10] and that would have a formal semantics. The language is supported by a software system that includes an execution engine, a graphical editor and a worklist handler. The system is available as Open source software under the LGPL license. Figure 2.6 illustrates a model for the scholarship variant process using YAWL notation, where the atomic tasks (denoted as squares) can be possibly complemented with control flow elements such as AND-split, AND-join, XOR-split, XOR-join, OR-split or OR-join.

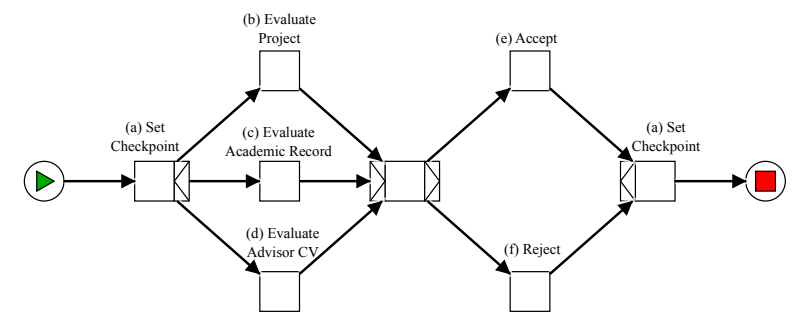

Figure 2.6: YAWL for scholarship variant process of Figure 2.3.

\footnotetext{
${ }^{1}$ For the sake of clarity, BPMN notation is abused representing an activity without label.
} 



\section{Part II}

\section{Precision in Conformance Checking}





\section{Chapter 3}

\section{Precision based on the Log}

\subsection{Introduction}

In the last years, the use within organizations of Process-Aware Information Systems (PAIS) and Business Process Management technologies (BPM) has experimented an exponential growth. Increasingly, organizations are investing vast amounts of time and resources on modeling their business processes [41, 102]. Process models are used to provide insights, analyze, simulate and improve the processes, and all conclusions obtained rely on how faithfully these models describe the reality [4]. Conformance checking techniques compare recorded process executions in terms of event logs with process models to quantify how good these models are. Checking conformance is a complex multi-dimensional task that involves the dimensions of fitness, precision, generalization and simplicity [4]. While fitness evaluates whether the behavior in the log is captured in the model, precision evaluates how much behavior is allowed by the model which is unlikely given the observed behavior in the log. Precise models are desirable: when the model becomes too general allowing for more behavior than necessary, it becomes less informative as it no longer describes the actual process. The extreme case is the flower model, shown in Figure 3.1, a model that allows for the execution of activities $a-i$ in any order. The fitness in this case is perfect because it captures any possible log over the activities $a-i$, but the precision is extremely poor and the model provides no insights on the process. Many approaches in the literature relate to the fitness dimension, e.g., [20, 16, 21, $33,60,46,47,71,80,85,4,8,100,1,54]$. In contrast, few are the ones proposed to measure precision, e.g. $[19,80,97,59,47]$.

In this chapter we present an approach to estimate precision between an event log and a process model based on detecting and quantifying escaping arcs, i.e., the points where the

- The materials reported in this chapter are partially published in [62] - 


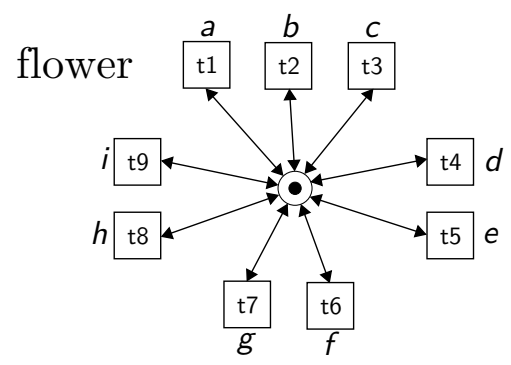

$$
\begin{aligned}
& \mathrm{a}=\text { Start Checkpoint } \\
& \mathrm{b}=\text { Register Low-value Claim } \\
& \mathrm{c}=\text { Register High-value Claim } \\
& \mathrm{d}=\text { Check Policy } \\
& \mathrm{e}=\text { Consult Expert } \\
& \mathrm{f}=\text { Check Liability } \\
& \mathrm{g}=\text { Complete Low-value Claim } \\
& \mathrm{h}=\text { Complete High-value Claim } \\
& \mathrm{i}=\text { Close Checkpoint }
\end{aligned}
$$

Figure 3.1: Flower model for the activities $a-i$.

model allows more behavior than the one observed in the log. The remainder of this chapter is organized as follows. Section 3.2 outlines the importance of precision checking, specially in real-life scenarios. Related work and other strategies for precision checking are presented in Section 3.3. Section 3.4 introduces the concept of precision checking based on escaping arcs, a procedure decomposed in two steps: constructing the observed behavior from the log - presented in Section 3.5 - and incorporating in it information about the modeled behavior - presented in Section 3.6. Section 3.7 proposes a metric to estimate the precision, and Section 3.8 presents a mechanism to collect the points of imprecision for future use. In Section 3.9 we provide some experimental results, and in Section 3.10 we discuss about the limitations and possible extensions of the proposed approach. Finally, Section 3.11 concludes the chapter.

\subsection{The Importance of Precision}

From a theoretical point of view, the need for a multi-dimensional conformance, and specially the necessity of measuring the precision, has been plenty justified and illustrated in the literature [80, 33]. The flower model represents the theoretical worst scenario concerning precision.

But besides the theory, the importance of precision is also a key element of real-life scenarios. The lack of precision on the models used in real-life systems may reduce the effectiveness of those systems. Some examples of those scenarios are:

- Workflow Management Systems: Workflow Management Systems and other processaware information systems [41] make use of process models to set-up and monitoring the defined sequence of tasks in a process. A precise model would guide the system, limiting and suggesting the next task to be performed, improving the efficiency. On the other hand, an imprecise model would be a poor guide, allowing too much tasks at the same time, and not giving real useful information.

- Regulations and Certifications: Regulations, such as the Sarbanes-Oxley (SOX) Act [86], 
enforce the documentation of processes, while quality certification, such as the ISO 9000 standards, requires the documentation and monitoring of all key processes to ensure their effectiveness. The use of overgeneralized models as part of the audition and certification procedure may provide an inexact vision of the processes, failing the auditing process [14].

- Communication: Models are used to communicate and gain insight into the analyzed processes, for example, models illustrating the reworks done in the process. Models allowing for more behavior than the one seen in reality would difficult the understanding of the processes, indicating a possible rework path that never happened.

- Simulation: Process models are used for simulating possible future scenarios, and to take decisions according to results obtained. The use of imprecise models would result in overgeneralized simulations, with a lot of non realistic scenarios, limiting the effectiveness of the conclusions.

- Abstraction: Some systems require a high level of flexibility. Health-care systems are good examples of flexible systems, where the path followed by two patients is never the same. In those cases, the need for precision is less crucial. However, extremely overgeneralized models would mask possible path restrictions, compromising the usefulness of the system.

In conclusion, the need for achieving precise models is becoming more crucial in nowadays systems, for bothcarahuevo conformance checking and process discovery techniques.

\subsection{Related Work}

In contrast with the fitness dimension, few are the approaches proposed in the literature that address, totally or partially, the precision checking. In [47], Greco et al. propose a metric - soundness - to estimate the precision by calculating the percentage of traces in the log that can be generated by the given model. Medeiros et al. [59] defines a metric -behavioral precision- to measure the precision between two models and a log, evaluating how much of the first model behavior is covered by the second. This measure is used within the Genetic Miner $[60,58]$-a discovery approach based on evolutionary algorithms- to evaluate the quality of the population obtained. Goedertier et al. [46] introduces the use of artificial negative examples to measure the precision between an event log and a process model. The way of generating those negative examples was later improved by De Weerdt et al. [97] and Vanden Broucke et al. [27]. Finally, Van Dongen et al. [40, 39] addresses the precision dimension between two models without a $\log$, based on the similarity of their structures.

However, given this chapter goal of checking precision between a process model and an event log, Rozinat et al. [80] can be seen as the seminal work, later extended in [79]. In [80], Rozinat et al. present several metrics to estimate the four dimensions of conformance 


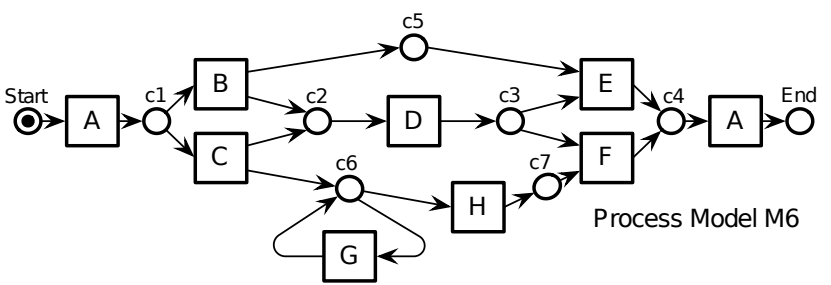

\begin{tabular}{|c|c|}
\hline No. of Instances & Log Traces \\
\hline 1207 & ABDEA \\
145 & ACDGHFA \\
56 & ACGDHFA \\
23 & ACHDFA \\
28 & ACDHFA \\
\hline
\end{tabular}

Analyze whether activities in the model
Always $(A)$, Never $(N)$, or Sometimes (S) Always (A), Never
follow each other

\begin{tabular}{|c|c|c|c|c|c|c|c|c|}
\cline { 2 - 8 } \multicolumn{1}{c}{ F } & A & B & C & D & E & F & G & H \\
\hline A & A & S & S & A & S & S & S & S \\
\cline { 2 - 8 } B & A & N & N & A & A & N & N & N \\
\cline { 2 - 8 } C & A & N & N & A & N & A & S & A \\
\cline { 2 - 8 } D & A & N & N & N & S & S & S & S \\
\hline E & A & N & N & N & N & N & N & N \\
F & A & N & N & N & N & N & N & N \\
G & A & N & N & S & N & A & S & A \\
H & A & N & N & S & N & A & N & N \\
\hline
\end{tabular}

(a) "Follows" relations from model perspective
Analyze whether events in the log actually Always (A), Never (N), or Sometimes (S) followed each other

Figure 3.2: 'Follows' relations used to compute the behavioral appropriateness $\left(a_{B}^{\prime}\right)$. Image taken from Process Mining: Conformance and Extension by Anne Rozinat [79].

checking. In particular, they present the advanced behavioral appropriateness $\left(a_{B}^{\prime}\right)$, a metric designed to measure the precision between a Petri net and an event log. The metric is based on deriving global 'Follows' and 'Precedes' activity relations from both a model and a log perspective, i.e., given all pairs of activities $x$ and $y$, determine whether they either always (i.e., in all traces), never (i.e., in none of the traces), or sometimes (i.e., in some but not all traces) follow/precede each other. Figure 3.2 shows an example of 'Follows' relation, taken from [79]. Because precision relates to those situations where the model has more variability than the event log, the idea of the metric is compare the behavior allowed by the model and the behavior observed in the log based on how many elements are contained in the sometimes follows and sometimes precedes relations once we superpose two the matrices.

The aforementioned technique has some limitations. First, precision is based on activity 
relations with a high level of abstraction but not precise points of deviation, i.e., only three categories are considered: always, never and sometimes. Although this can be useful to get insights on the precision from a high level, it becomes a limitation when it comes to detect exactly those precision problems. Moreover, the correct categorization of follows/precedes relations is not guaranteed when the traces contain loops [79]. Finally, building the relations from a model point of view requires the analysis of the model task sequences based on a state space analysis or an exhaustive model simulation. This limits the applicability of the approach to examples of low complexity, or forces the use of approximations to alleviate the complexity.

\subsection{Precision based on Escaping Arcs}

This chapter presents an approach to check the precision between a process model and an event $\log$ that enable a analyst to both measure the precision dimension and diagnose the causes of the precision problems and where are located. In particular, we aim for:

- A precision based on potential points of improvement. The precision dimension can be viewed from different angles. One can estimate the precision of a system as the difference between the behavior allowed by the model and the behavior observed in the log. Instead, we propose a different way of estimating precision based on identifying all precision problems and quantifying the effort need to correct them achieving a perfectly precise system.

- A technique that does not require an exhaustive model state-space exploration. Because the state space of a model can grow exponentially, state-based analysis techniques may be problematic with respect to computational complexity [77]. Therefore, any approach involving an exhaustive exploration of the model state-space, such as [80], sees its applicability compromised for complex cases, often found in reality.

- An approach to identify precision problems with a fine granularity. In order to help in the process diagnosis, the approach should be able to detect the exact precision points of improvement in the model.

- A mechanism to use the precision results for analysis and process improvement. Besides a metric, any approach proposed should consider the possibility of collecting all precision anomalies detected in a format suitable for analysis using process mining techniques, or to be used within the continuous cycle of improvement and correction of the process.

The approach proposed in this chapter addresses these goals and presents a precision checking technique based on the detection and collection of escaping arcs between a log and a model. The escaping arcs define those crucial points where the model starts to deviate 


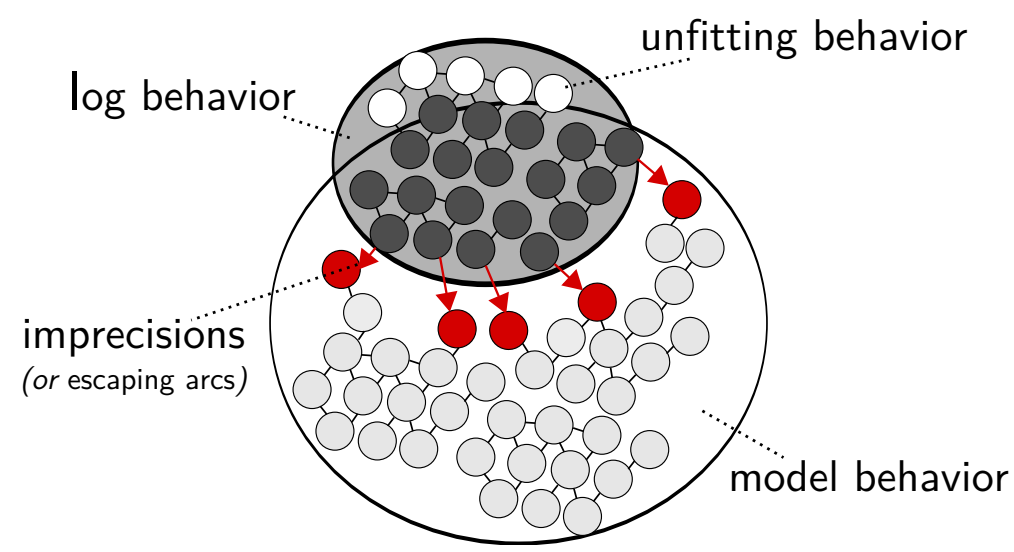

Figure 3.3: Overview of observed behavior, modeled behavior, and escaping arcs.

from the behavior observed in the log. Figure 3.3 shows an overview of the escaping arcs. On the one hand, the behavior observed in the log is determined by a set of states. On the other hand, a possibly different set of states determine the behavior represented by the model. When compared, both sets usually overlap. In an ideal situation, both set of states will be equal, representing a complete precise scenario. However, this is not usually the case. In most of the cases, some of the observed states are not modeled, representing some unfitting behavior. Symmetrically, there is also the situation where that the model includes behavior not observed, denoting an imprecise model. The 'border' between the observed behavior and the modeled behavior determines a set of escaping arcs, points reaching a state modeled but not observed in the $\log$.

The escaping arcs depend entirely on how the observed and modeled states are determined. The technique proposed in this chapter is decomposed into the following steps (cf. Figure 3.4):

1. Constructing the observed behavior: First, the behavior in the log is analyzed, extracting state information from it, constructing an automaton representing the observed behavior. This step is explained in detail in Section 3.5.

2. Incorporating modeled behavior: Second, the observed behavior automaton is enhanced incorporating information about the modeled states. This step does not require an exhaustive exploration of the modeled behavior, but the exploration is restricted to the 'border' between observed and modeled behavior. This step is explained in detail in Section 3.6.

3. Detecting escaping arcs and estimating precision: The escaping arcs of a state refer to those points where the model allows more behavior than the one actually recorded 


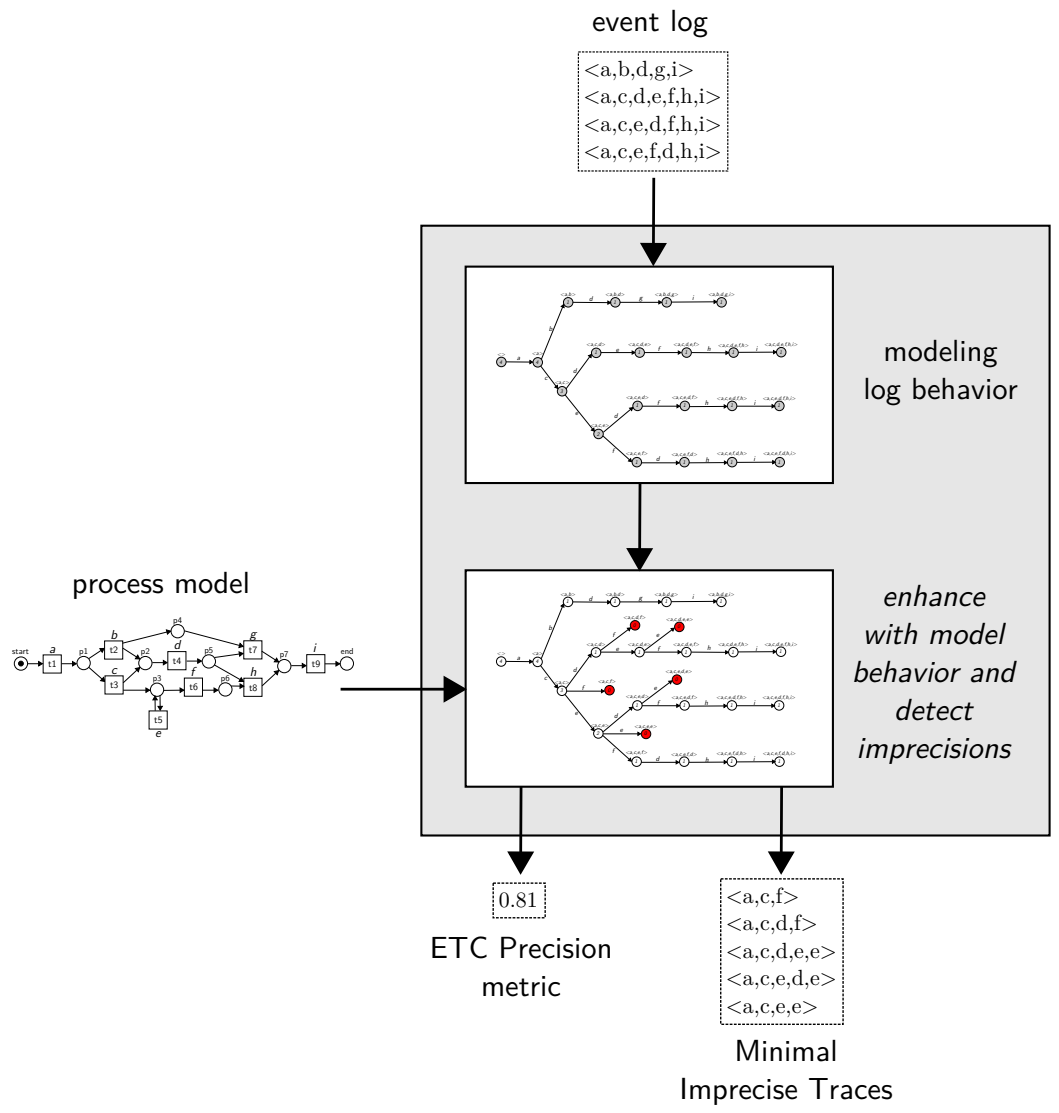

Figure 3.4: Route map of the precision based on escaping arcs.

in the log. The number of escaping arcs and their location are used to estimate the precision of the whole system in terms of a metric. This step is explained in detail in Section 3.7.

4. Collecting minimal imprecise traces: Finally, all the precision problems detected are collected in terms of an event log, describing the minimal traces leading to a precision problem. This step is explained in Section 3.8. 


\subsection{Constructing the Observed Behavior}

In this section we present the first necessary step for the precision checking proposed in this section: determining the states conforming the observed behavior by means of an automaton. The state definition proposed in this section is based on the prefixes of the traces, i.e. given a $\log$ trace $\sigma$ reflecting an instance of the process, the prefixes of $\sigma$ determine the states reached by the system during that execution.

Definition 3.1 (Prefixes of a $\log$ ). Let $L \in \mathcal{B}\left(A^{*}\right)$ be an event log, where $\sigma=\left\langle a_{1}, a_{2}, \ldots, a_{n}\right\rangle \in$ $L$ is a trace of the log. $\bullet(\sigma)$ is the set of prefixes of the trace $\sigma$, i.e., $\bullet(\sigma)=\left\{\left\langle a_{1}, a_{2}, \ldots, a_{m}\right\rangle \mid\right.$ $m \leq n\} . \bullet(L) \in \mathcal{B}\left(A^{*}\right)$ is the multiset of prefixes of the $\log L$, i.e., $\bullet(L)=\biguplus_{\sigma \in L} \bullet(\sigma)$.

Notice that, the empty sequence \langle\rangle and the complete sequence are prefixes of any trace. Let us consider for example the trace $\sigma=\langle a, b, d, g, i\rangle$ of $\log L_{1}$ in Figure 3.5. The prefixes $\bullet(\sigma)=\{\langle\rangle,\langle a\rangle,\langle a, b\rangle,\langle a, b, d\rangle,\langle a, b, d, g\rangle,\langle a, b, d, g, i\rangle\}$ represent the states reached by the system during the execution recorded in $\sigma$. The set of prefixes resulting of applying this definition to all the traces in $L_{1}$ represents the set of observed states of the whole log.
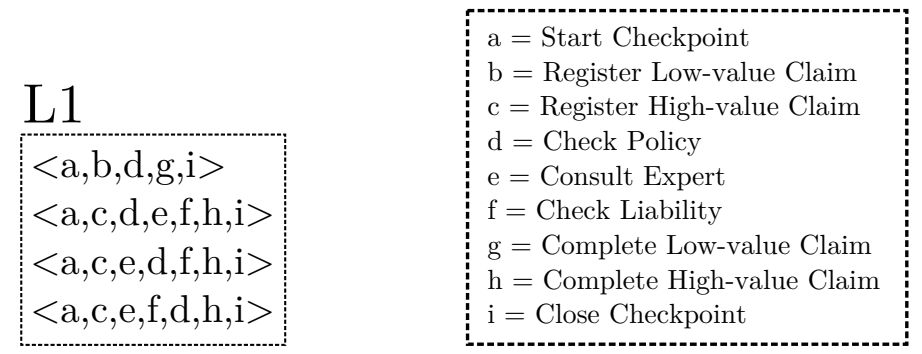

Figure 3.5: Event $\log L_{1}$ for a liability insurance claim process.

The state information extracted from a log is used to construct a compact representation in terms of an automaton. The prefix automaton contains the states, the transitions between the states, and the weight of a state within the process.

Definition 3.2 (Prefix Automaton of the $\log$ ). Let $L \in \mathcal{B}\left(A^{*}\right)$ be an event log, where $A$ is the set of activities. We define the prefix automaton of the $\log$ as an extension of the transition system definition $\mathcal{A}_{L}=(S, A, \nearrow, \omega,\langle\rangle)$ such that:

- the set of states correspond to the set of prefixes of the log, i.e., $S=\{\sigma \mid \sigma \in \bullet(L)\}$.

- the set of labels correspond to the set of activities of the log, i.e., $A$.

- the arcs $\nearrow \subseteq(S \times A \times S)$ define the concatenation between prefixes and activities, i.e., $\nearrow=\{(\sigma, a, \sigma \cdot\langle a\rangle) \mid \sigma \in S \wedge \sigma \cdot\langle a\rangle \in S\}$. 
- the function that determines the weight of a state is determined by the number of occurrences of the state in the multiset of prefixes of the $\log$, i.e., $\omega(\sigma)=\bullet(L)(\sigma)$.

- the initial state corresponds with the empty prefix \langle\rangle .

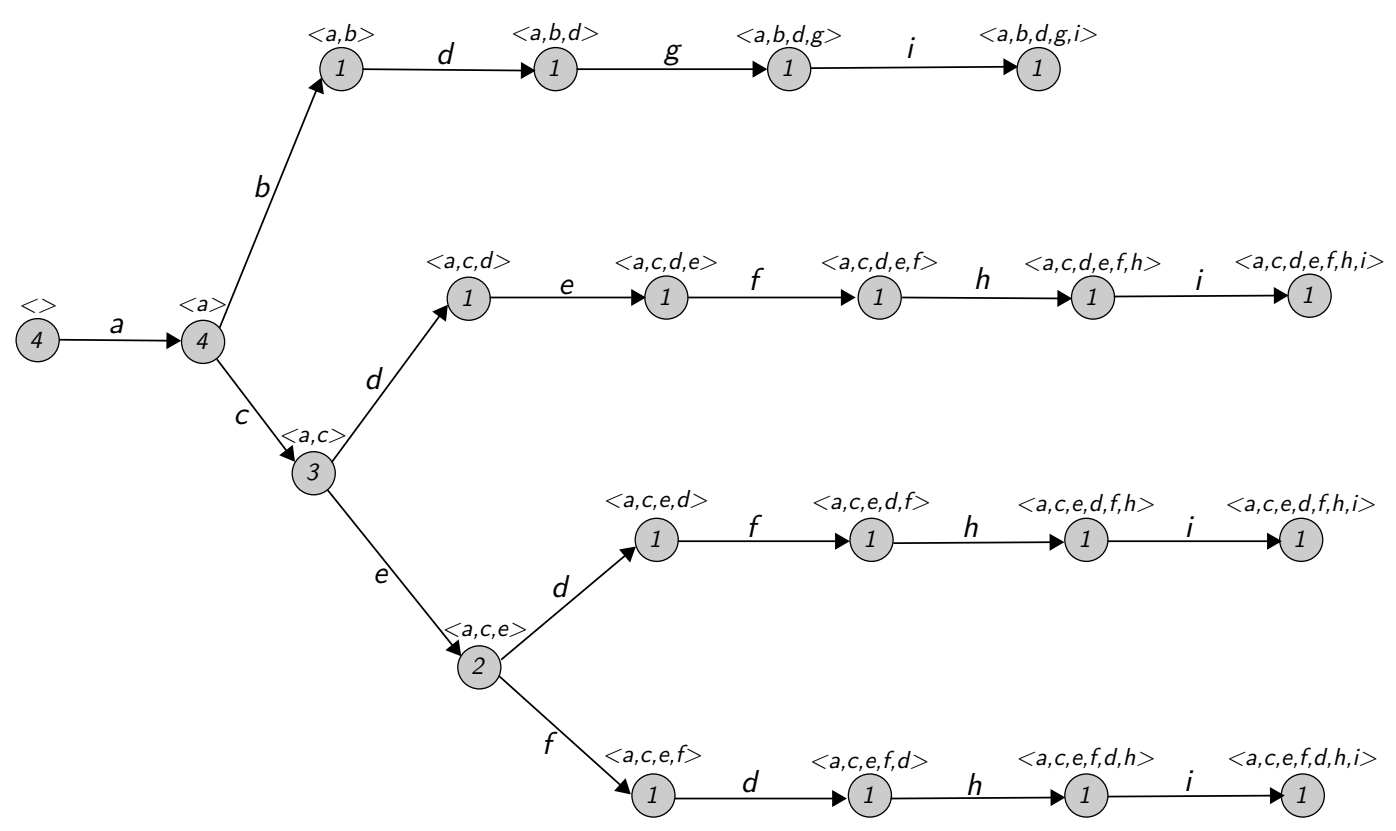

Figure 3.6: Prefix automaton $\mathcal{A}_{L_{1}}$ for the event $\log L_{1}$.

Figure 3.6 illustrates the construction of the prefix automaton for the $\log L_{1}$. Each prefix of $L_{1}$ identifies a state. The number in the states represent the weight function. For instance, the state $\langle a\rangle$ has a weight of $\omega(\langle a\rangle)=4$ because it appears four times in $\bullet(L)$. On the other hand, $\langle a, b\rangle$ appears only once, i.e., $\omega(\langle a, b\rangle)=1$. The initial state corresponds with the prefix \langle\rangle .

In [12], the authors proposed a configurable approach to construct a transition system from an event log. The definition of state and the events considered to build a state can be parametrized depending on the abstraction level desired. In particular, the parameters past, sequence, and no horizon will result in a transition system with the same characteristics as the prefix automaton proposed in this section. In Chapter 5 we consider the use of other values for the parameters and their effects on the precision checking. 


\subsection{Incorporating Modeled Behavior}

In this section we use the prefix automaton representing the observed behavior to restrict the exploration of the modeled behavior. Let us consider, for example, the model $S N_{1}$ in Figure 3.7, presented in terms of a system net, with [start] and [end] as initial and final markings respectively. ${ }^{1}$ The system net is a possible model for the insurance process observed in the $\log L_{1}$. Given a state of the prefix automaton, we analyze the set of activities possible when the model reaches that state. For example, let us consider the state $\langle a\rangle$ of the prefix automaton $\mathcal{A}_{L_{1}}$. Analyzing the set of complete activity sequences of $S N_{1}$ we realize that, once the model reaches the state $\langle a\rangle$, only two activities can follow: $b$ and $c$.

For the sake of clarity, in this section we assume that the log perfectly fits the model. That is to say that all traces of the $\log L$ are included in the set of complete activity sequences of the model $M$, i.e. $\forall \sigma \in L: \sigma \in \phi(M)$. Consequently, any prefix of the trace $\sigma$ represents a state reachable by the model. In Section 3.10 we study the relaxation of this assumption, and how the theory proposed is extended accordingly.
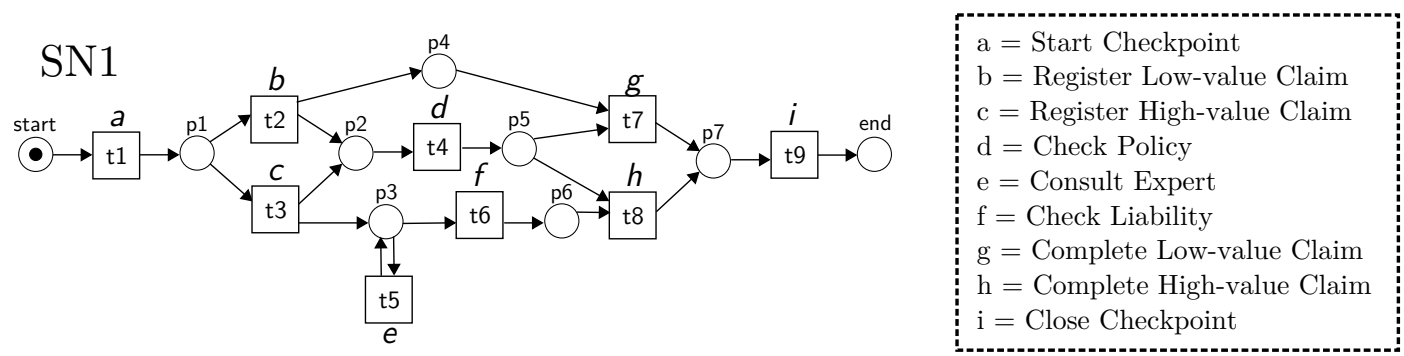

Figure 3.7: System net $S N_{1}$ modeling the liability insurance claim process.

Given a prefix automaton from the log, we analyze the activities allowed by the model in each log state, and we incorporate new states denoting those activities modeled but never observed in the log. Let us consider for example the automaton $\mathcal{A}_{L_{1} S N_{1}}$ in Figure 3.8, result of enhancing $\mathcal{A}_{L_{1}}$ with modeled behavior of $S N_{1}$. The states in white represent states both in the log and model behavior. The colored states represent the new states introduced, that belong only to modeled behavior but never observed in the log. The weight of these new states is 0 , denoting that they are not observed in the log. For example, the state $\langle a, c\rangle$ represents a state both in the $\log L_{1}$ and the model $S N_{1}$, but $\langle a, c, f\rangle$ is a state only of the model (i.e., $\langle a, c, f\rangle$ is not a prefix of $L_{1}$ ).

Formally, the enhancement of the observed prefix automaton with modeled behavior is defined as follows:

\footnotetext{
${ }^{1}$ Notice that we use system nets in this section for illustrative purposes only, while the theory refers to any process model.
} 


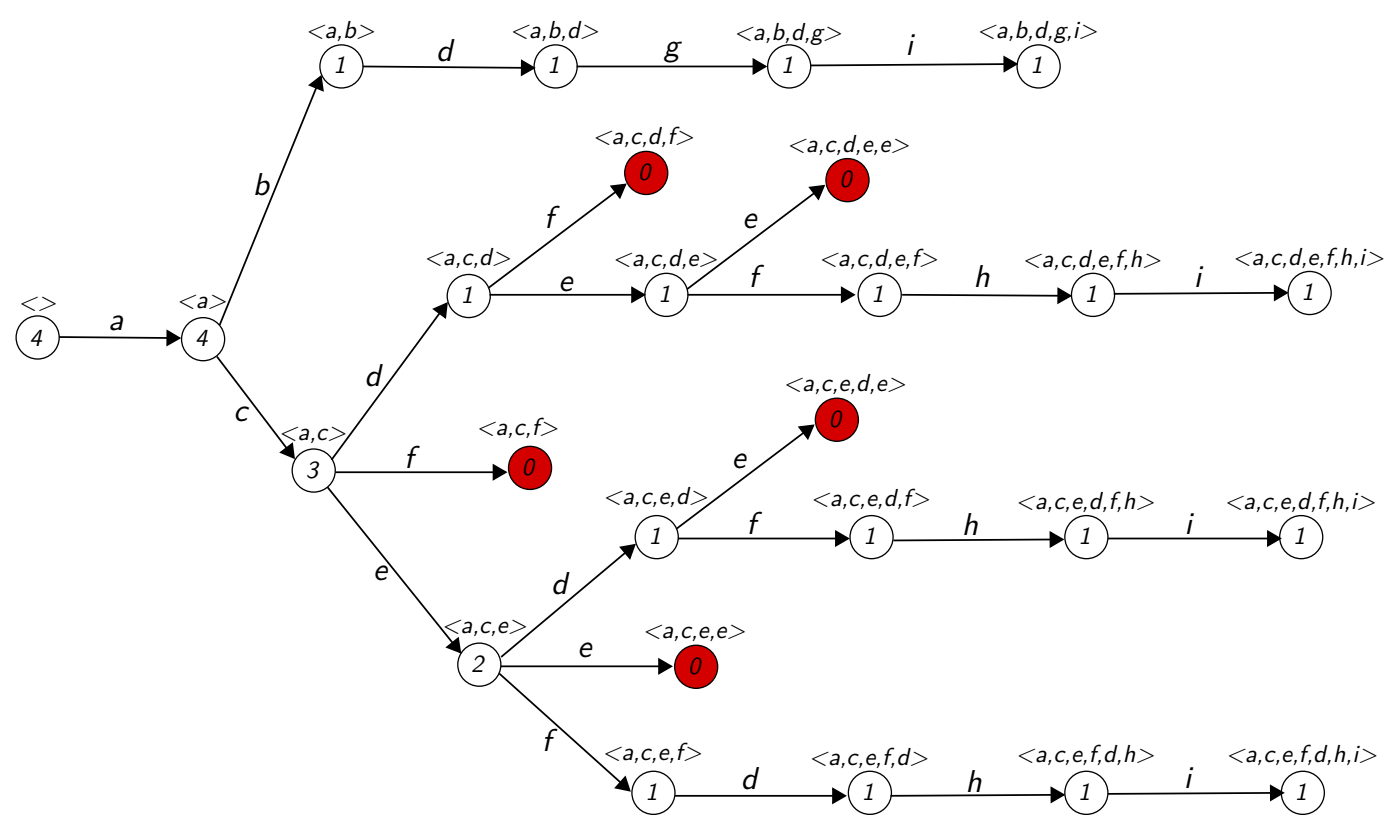

Figure 3.8: Prefix automaton $\mathcal{A}_{L_{1} S N_{1}}$ of the event $\log L_{1}$ enhanced with the modeled behavior of the system net $S N_{1}$.

Definition 3.3 (Prefix Automaton of the Observed Behavior Enhanced with the Modeled Behavior). Let $L \in \mathcal{B}\left(A^{*}\right)$ be an event log, where $A$ is a set of activities. Let $M$ be a process model, where $\phi(M)$ is the set of complete activity sequences of $M$. Similar to $\bullet(L), \bullet(\phi(M))$ represents the prefixes of the complete activity sequences of $M$, i.e., $\bullet(\phi(M))=\biguplus_{\sigma \in \phi(M)} \bullet(\sigma)$. We define the prefix automaton of the observed behavior enhanced with modeled behavior $\mathcal{A}_{L M}=(S, A, \nearrow, \omega,\langle\rangle)$ such that:

- Let $S_{L M}=\{\sigma \mid \sigma \in \bullet(L) \cap \bullet(\phi(M))\}$ be the states that are in both observed and modeled behavior. Let $S_{M}=\{\sigma \mid \sigma \notin \bullet(L) \wedge \sigma \in \bullet(\phi(M))\}$ be states that are in the modeled behavior but not in the observed behavior ${ }^{2}$. The set of states $S$ of the enhanced automaton is the union $S=S_{L M} \cup S_{E}$, where $S_{E} \subseteq S_{M}$ are the states only in the modeled behavior that come preceded by a state in both behaviors, i.e., $S_{E}=\left\{\sigma \cdot\langle a\rangle \mid \sigma \in S_{L M} \wedge \sigma \cdot\langle a\rangle \in S_{M}\right\}$.

- The set of labels correspond to the set of activities of the process, i.e., $A$.

- The arcs $\nearrow \subseteq(S \times A \times S)$ define the concatenation between states and activities, i.e., $\nearrow=\{(\sigma, a, \sigma \cdot\langle a\rangle) \mid \sigma \in S \wedge \sigma \cdot\langle a\rangle \in S\}$.

\footnotetext{
${ }^{2} S_{L}=\{\sigma \mid \sigma \in \bullet(L) \wedge \sigma \notin \bullet(\phi(M))\}$ is not possible because we assume perfect fitness (cf. Section 3.10.)
} 
- The function that determines the weight of a state is determined by the number of occurrences of the state in the multiset of prefixes of the $\log P$ with $P=\bullet(L)$, or 0 if the state does not appear on the log:

$$
\omega(\sigma)= \begin{cases}P(\sigma) & \text { if } \sigma \in S_{L M} \\ 0 & \text { if } \sigma \in S_{E}\end{cases}
$$

- The initial state corresponds with the empty prefix \langle\rangle .

Notice that, although the definition of the enhanced prefix automaton considers the set of complete activity sequences $\phi(M)$ of a model $M$, in practice the approach proposed does not require computing all the sequences in advanced. In the previous example, for the system net $S N_{1}$ the set of complete activity sequences is infinite due the transition $t_{5}$. Instead, in executable process models such as Petri nets or BPMN, the sequences can be constructed progressively on demand. For example, given $S N_{1}$ and the state $\langle a\rangle$, we analyze the marking reached after firing $t_{1}$ (i.e., $\left[p_{1}\right]$ ) and the activities allowed (i.e., $b$ and $c$ ), being $\langle a, b\rangle$ and $\langle a, c\rangle$ both sequences possible in the model. Notice that, the presence of invisible and duplicate transitions may arise some potential indeterminism about the marking reached after a given prefix, i.e., the same sequence of activities may match several tasks sequences, reaching different markings, and therefore, allowing different set of activities. For the sake of clarity in this section we assume determinism. In Section 3.10 we explore the relaxation of this assumption and the consequences that this may produce.

Moreover, the proposed approach does not require the complete exploration of model behavior. Unlike other approaches in the literature that require an exhaustive exploration of the model state space (e.g., [80]), the proposed approach restricts the exploration to the boundaries of the log state space. Only the border between the behavior observed in the $\log$ and the model behavior is explored. For example, given $L_{1}$ and $S N_{1}$, the trace $\langle a, c, d, e, e, e, e, e, f, h, i\rangle$ included in the model behavior is never explored.

\subsection{Detecting Escaping Arcs and Evaluating Precision}

Given an event $\log L$ and process model $M$, the prefix automaton $\mathcal{A}_{L M}$ contains the juxtaposition of both observed and modeled behaviors, and the border defined between them. Each state of the automaton represents a state reached by the system during the execution recorded in the log. The precision checking approach proposed in this chapter bases its estimation on comparing, for each one of these states, the activities allowed by the model and the activities that where recorded in the log.

Definition 3.4 (Observed and Modeled Arcs). Let $\mathcal{A}_{L M}=(S, A, \nearrow, \omega,\langle\rangle)$ be the prefix automaton of the $\log L$ enhanced with the behavior of the model $M$. Let $S_{L M}$ be the states that are in both observed and modeled behavior, and let $S_{M}$ be the states only in the modeled behavior. Let $\sigma \in S_{L M}$ be a state of the automaton. obs $(\sigma)=\left\{\left(\sigma, a, \sigma^{\prime}\right) \in \nearrow \mid \sigma \in S_{L M} \wedge \sigma^{\prime} \in\right.$ 
$\left.S_{L M}\right\}$ represent those arcs whose activities were executed and consequently recorded on the $\log L$ when the system was on the state $\sigma \cdot \bmod (\sigma)=\left\{\left(\sigma, a, \sigma^{\prime}\right) \in \nearrow \mid \sigma \in S_{L M} \wedge \sigma^{\prime} \in\right.$ $\left.S_{L M} \cup S_{M}\right\}$ represent those arcs whose activities are modeled and consequently allowed by the model $M$ when the system was on the state $\sigma$. Similarly, we refer as observed $/$ modeled states of $\sigma$ those states reached through an observed/modeled arc from $\sigma$, and observed $/$ modeled activities of $\sigma$ those activities used in the observed/modeled arcs from $\sigma$.

For example, let us consider the state $\langle a, c\rangle$ of the automaton $\mathcal{A}_{L_{1} S N_{1}}$. The activities modeled by the model $S N_{1}$ in that state are $d, e$ and $f$. On the other hand, the activities observed in the same state are only $d$ and $e$. Notice that in this section we are under the assumption that the $\log$ perfectly fits the model. Therefore, observed $(\sigma) \subseteq$ modeled $(\sigma)$ for all states $\sigma \in S_{L M}$.

An escaping arc denotes a point where the behavior of the process model allows more than what has been actually observed in the log, i.e., an arc that escapes from the log behavior.

Definition 3.5 (Escaping Arcs). Let $\mathcal{A}_{L M}=(S, A, \nearrow, \omega,\langle\rangle)$ be the prefix automaton of the $\log L$ enhanced with the behavior of the model $M$, where $\sigma \in S_{L M}$. The set of escaping arcs of the state $\sigma$ is determined by the difference between the arcs modeled and the arcs allowed on the state, i.e., $\operatorname{esc}(\sigma)=\bmod (\sigma) \backslash \operatorname{obs}(\sigma)$. Similarly, we refer to the activities used to escape and the states reached, as escaping activities and escaping states, respectively. The set of all escaping arcs of the automaton is denoted as $\operatorname{esc}\left(\mathcal{A}_{L M}\right)$.

Following with the example state $\langle a, c\rangle$ of the automaton in $\mathcal{A}_{L_{1} S N_{1}}$, there is only one escaping activity in that state: $f$. In other words, when the system was in the state $\langle a, c\rangle$, all activities allowed by the model in that point have been observed, except $f$. Notice that, by construction of the automaton, the escaping activities are considered globally, i.e., all the traces in the log are considered as a whole to compute the set of reflected activities, instead of analyzing trace by trace independently. For example, given the state $\langle a, c\rangle$ in $\mathcal{A}_{L_{1} S N_{1}}$, the activity $d$ is reflected in the second trace of $L_{1}$, and the activity $e$ is reflected in the third and forth traces of the $\log$.

In our view, a precise model is one that does not contain escaping arcs, i.e., for each state it only models the behavior observed on the log. A model where almost all the behavior allowed represents an escaping opportunity must be considered highly imprecise. In that sense, we define a metric -ETC Precision-designed to measure the precision between a model and a log, based on the escaping arcs. On the one hand, the metric quantifies the degree of escaping arcs of the automaton. This value is weighted according to the weight of the state where each escaping arc is located, i.e., escaping arcs in more frequent states have more impact in the metric that those who appear in infrequent and barely used parts of the process. On the other hand, the metric measures the modeled behavior on the automaton, weighted also according to the weights of the states. The metric defines the precision between a log and a model as the relation between the escaping behavior versus the modeled behavior.

Definition 3.6 (ETC Precision). Let $\mathcal{A}_{L M}=(S, A, \nearrow, \omega,\langle\rangle)$ be the prefix automaton of the $\log L$ enhanced with the behavior of the model $M$. The metric ETC Precision estimates 
the precision of the system comparing, for each state in $S_{L M}$, the number of escaping arcs with the number of modeled arcs. The numbers are weighted according to the importance of the state. Formally:

$$
\operatorname{etc}_{p}\left(\mathcal{A}_{L M}\right)=1-\frac{\sum_{\sigma \in S_{L M}} \omega(\sigma) \cdot|\operatorname{esc}(\sigma)|}{\sum_{\sigma \in S_{L M}} \omega(\sigma) \cdot|\bmod (\sigma)|}
$$

Let us consider, for example, the automaton $\mathcal{A}_{L_{1} S N_{1}}$. The automaton contains 21 states in $S_{L M}$, denoted in white. For each one of those states we compute the number of escaping arcs and the number of modeled arcs, and we weight them according to the weight of each state.

$$
\begin{aligned}
\operatorname{etc}_{p}\left(\mathcal{A}_{L_{1} S N_{1}}\right)=1- & \\
& \frac{4 \cdot 0+4 \cdot 0+1 \cdot 0+1 \cdot 0+1 \cdot 0+1 \cdot 0+\mathbf{3} \cdot \mathbf{1}+\mathbf{1} \cdot \mathbf{1}+\mathbf{1} \cdot \mathbf{1}+\cdots}{4 \cdot 1+4 \cdot 2+1 \cdot 1+1 \cdot 1+1 \cdot 1+1 \cdot 0+3 \cdot 3+1 \cdot 2+1 \cdot 2+\cdots} \\
& \frac{\cdots 1 \cdot 0+1 \cdot 0+1 \cdot 0+\mathbf{2} \cdot \mathbf{1}+\mathbf{1} \cdot \mathbf{1}+1 \cdot 0+1 \cdot 0+1 \cdot 0+1 \cdot 0+\cdots}{\cdots 1 \cdot 1+1 \cdot 1+1 \cdot 0+2 \cdot 3+1 \cdot 2+1 \cdot 1+1 \cdot 1+1 \cdot 0+1 \cdot 1+\cdots} \\
& \cdots 1 \cdot 0+1 \cdot 0+1 \cdot 0 \\
& \frac{\cdots 1 \cdot 1+1 \cdot 1+1 \cdot 0}{\cdots \cdot 1 \cdot 1+\frac{8}{43}}=1-0.19=0.81
\end{aligned}
$$

The etc $c_{p}$ value for the automaton $\mathcal{A}_{L_{1} S N_{1}}$ between $L_{1}$ and $S N_{1}$ is 0.81 , denoting a moderate precision degree of $81 \%$.

Taking a look at the literature one can see that the intuitive notion of precision is difficult to capture in a metric. Comparing a process model and an even log always allows for different interpretations and a wide range of metrics can defined. Facing so much uncertainty it is wise to impose some requirements to ensure the usefulness of any measure proposed. In [81] the authors present a list, based on [56], with five properties any conformance metric proposed should satisfy: validity, stability, analyzability, reproducibility and localizability. In the following part we analyze those properties and we provide a brief justification on how ETC Precision metric fulfills them:

- Validity Validity means that the measure and the property to measure must be sufficiently correlated with each other.

As it has been motivated, there is a direct relation between the precision of a system and the escaping arcs detected, justifying its validity. An increase in the precision degree is reflected in the number and importance of the escaping arcs, and thus, producing an increment on the metric value.

- Stability Stability means that the measure should be stable against manipulations of minor significance, i.e., be as little as possible affected by properties that are not measured. 
The approach proposed is defined at a activity level, i.e., the observed behavior is compared with the modeled behavior independently from the structural properties of the models. Two models with different structure but modeling the same behavior will result in the same metric value. This makes even possible to compare models defined using different notations, e.g., Petri nets and BPMN. The metric is defined such that the context of the precision problem is taken into account, but not the position, i.e., two states with the same weight will have the same importance in the metric no matter where they are located. Notice that, although the metric is defined to measure the precision dimension independently, possible problems and corrections in the fitness dimension may affect the stability of the precision results (cf. Section 3.10).

- Analyzability Analyzability, in general, relates to the properties of the measured values (e.g., whether they can be statistically evaluated). In the remainder, the emphasis is on the requirement that the measured values should be distributed between 0 and 1 , with 1 being the best and 0 being the worst value.

Notice that by definition $\operatorname{esc}(\sigma) \subseteq \bmod (\sigma)$, resulting in a metric that range from 0 to 1. Therefore, on the one hand an optimal value for precision is defined, i.e, 1 denotes that the observed behavior is precisely modeled. This is especially important as a stop condition in the context of an iterative approach looking for appropriate process models, such as genetic mining [60,32], but also for a human analyst as it indicates that there is no better solution available. Notice that, to achieve a value of 1 is not necessary to have all the modeled behavior observed in a single trace, i.e., the precision is considered globally, taking all the observed traces as a whole. Finally, the fact that the metric is normalized by the degree of allowed behavior in each state makes it possible to use it for comparing different pairs of model-log, even if they refer to different processes.

- Reproducibility Reproducibility means that the measure should be independent of subjective influence, i.e., it requires a precise definition of its formation.

The definition of the metric proposed is solid and formal, and there is no room for subjectivity. The same experiment can be reproduced several times and it will always output the same result. However, notice that the relation between activity sequences and tasks sequences of a process model may arise possible non-deterministic situations, requiring further assumptions in order to preserve the reproducibility of the approach (cf. Section 3.10).

- Localizability Localizability means that the system of measurement forming the metric should be able to locate those parts in the analyzed object that lack certain desirable (i.e., the measured) properties.

It is very important that a precision problem is not only reflected by the measured value but can also be located. In that sense, the escaping arcs captured in the automaton 
describe perfectly where the precision problems are, making possible for the business analyst to identify potential points of improvement. Additionally, in Section 3.8 we provide an additional mechanism to collect all those precision problems for a deeper analysis.

\subsection{Minimal Imprecise Traces}

The ETC Precision metric presented in the previous section provides a numeric measurement on the precision of the system. This value may be useful to measure the precision of several alternative models describing the same observed behavior, or to establish when a model becomes obsolete to represent an evolving process. However, in order to fully understand the causes of the precision problems, an analyst needs to be able to access the exact points of mismatch between the observed and modeled behaviors. The prefix automaton and its detected escaping arcs provide this information, and may be used to guide a deeper analysis into model and the log to understand their discrepancy. Some of the escaping arcs may represent meaningful abstractions that arise in the model and therefore no further action is required. Others, however, may suggest situations for which future actions over the process need to be carried out.

Additionally to the escaping arcs detected on the automaton, in this section we propose to collect all the precision anomalies in terms of logs and traces to be used later on for its analysis. Each escaping arc is represented by one minimal imprecise trace (mit), a sequence containing the minimal behavior observed until the escaping arc was available. All the minimal imprecise traces compose the minimal imprecise log.

Definition 3.7 (Minimal Imprecise Traces and $\log$ ). Let $\mathcal{A}_{L M}=(S, A, \nearrow, \omega,\langle\rangle)$ be the prefix automaton of the $\log L$ enhanced with the behavior of the model $M$. Let $\operatorname{esc}\left(\mathcal{A}_{L M}\right)$ define all the escaping arcs of the automaton. Given the escaping $\operatorname{arc}\left(\sigma, a, \sigma^{\prime}\right) \in \operatorname{esc}\left(\mathcal{A}_{L M}\right)$, its minimal imprecise trace is defined as $\operatorname{mit}\left(\left(\sigma, a, \sigma^{\prime}\right)\right)=\sigma^{\prime}$. The set of all minimal imprecise traces define the minimal imprecise log, i.e., $\operatorname{mil}\left(\mathcal{A}_{L M}\right)=\bigcup_{i \in \operatorname{escape}\left(\mathcal{A}_{L M}\right)} \operatorname{mit}(i)$.

In $\mathcal{A}_{L_{1} S N_{1}}$, there are five escaping arcs in the automaton, and thus, five are the minimal imprecise traces conforming the minimal imprecise log, shown in Figure 3.9. Notice that, by definition, all minimal imprecise traces fulfill a minimality criterion, i.e., their represent the minimal behavior before the deviation. In other words, all elements in the trace except the last one represent a behavior observed in the log, and the last one is the activity allowed by the model but not observed.

The representation of the imprecisions in terms of a minimal imprecise log opens the possibility to different analysis and uses. For example, all techniques and tools based in analyzing event logs can be used to gain insights into the precision problems. That includes most of the approaches in the field of process mining, e.g., discovery algorithms can be used to derive a model that represents the imprecise behavior. Some of the minimal imprecise traces can be used for process reparation, correcting the model to represent more precisely 

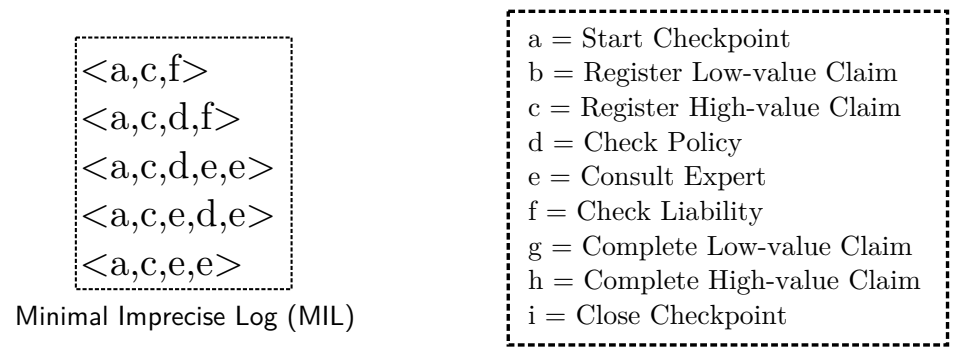

Figure 3.9: Minimal Imprecise $\log (\mathrm{MIL})$ for the automaton $\mathcal{A}_{L_{1} S N_{1}}$.

the observed behavior, e.g., transforming pairs of concurrent events in the model to ordered events [61]. Another option is to use the minimal imprecise $\log$ as input for the supervisory control theory [76], i.e., synthesizing a supervisor (i.e., another model synchronized with the original model) that restricts the behavior of original system such the imprecisions never occur. Finally, similar to [46, 97, 27], the minimal imprecise trace can be considered as negative examples, and can be used to enrich the original log in order to discover a more precise model.

\subsection{Experimental Results}

The technique presented in this paper, implemented as part of the ETConformance plug-in within ProM 6 [75], has been evaluated on existing public-domain benchmarks [74]. The purpose of this section is:

- Justify empirically the existence of a new metric to evaluate precision, i.e. demonstrate the novelty of the concept when compared to previous approaches.

- Show the capacity of the technique to handle large specifications.

Table 3.1a shows a comparison of the technique presented in this chapter with the technique presented in [80], implemented in ProM 5.2 as the Conformance Checker. The rows in the table represent benchmarks with small size (few traces). The names are shortened, e.g., GFA5 represents GroupedFollowsA5. We report the results of checking precision for both conformance checkers in columns under $a_{B}^{\prime}$ and $e t c_{p}$, respectively, for the small Petri nets obtained by the ILP miner [101] which derived Petri nets with fitness value one. For the case of our checker, we additionally provide the number of minimal imprecise traces $(|M I L|)$. We do not report CPU times since checking precision in both approaches took less than one second for each benchmark.

From Table (a) one can see that when the model describes precisely the log, both metrics provide the maximum value. Moreover, when the model is not a precise description of the 
log, only three benchmarks provide opposite results (GFBN2, GF121, GF121Skip). For instance, the GFI2ISkip benchmark $a_{B}^{\prime}$ is providing a significant lower value: this is because the model contains an optional loop that is always traversed in the log. This variability is highly penalized by simply observing the tasks relations. On the other hand, metric etc $c_{p}$ will only penalize the few situations where the escaping edges appear in the log.

\begin{tabular}{|l|r|rr|}
\hline Benchmark & $a_{B}^{\prime}$ & $e t c_{p}$ & $|M I L|$ \\
\hline GFA6NTC & 1.00 & 1.00 & 0 \\
GFA7 & 1.00 & 1.00 & 0 \\
GFA8 & 1.00 & 1.00 & 0 \\
GFA12 & 1.00 & 1.00 & 0 \\
GFChoice & 1.00 & 1.00 & 0 \\
GFBN1 & 1.00 & 1.00 & 0 \\
GFParallel5 & 1.00 & 0.99 & 11 \\
GFAL1 & 1.00 & 0.88 & 251 \\
\hline
\end{tabular}

\begin{tabular}{|l|r|rr|}
\hline Benchmark & $a_{B}^{\prime}$ & $e t c_{p}$ & $|M I L|$ \\
\hline GFl2lOpt & 1.00 & 0.85 & 7 \\
GFAL2 & 0.86 & 0.90 & 391 \\
GFDrivers & 0.78 & 0.89 & 2 \\
GFBN3 & 0.71 & 0.88 & 181 \\
GFBN2 & 0.59 & 0.96 & 19 \\
GFA5 & 0.50 & 0.57 & 35 \\
GFl21 & 0.47 & 0.75 & 11 \\
GFl21Skip & 0.30 & 0.74 & 10 \\
\hline
\end{tabular}

(a)

\begin{tabular}{|cc|c|rr|rrr|rr|rrr|}
\cline { 3 - 13 } \multicolumn{1}{c|}{} & $M_{T}$ & \multicolumn{4}{c|}{ Parikh } & \multicolumn{5}{c|}{ RBMiner } \\
\hline Benchmark & $|T S|$ & etc $c_{p}$ & $|P|$ & $|T|$ & etc $c_{p}$ & $|M I L|$ & CPU & $|P|$ & $|T|$ & etc $c_{p}$ & $|M I L|$ & CPU \\
\hline a22f0n00_1 & 1309 & 0.06 & 19 & 22 & 0.63 & 1490 & $0(0)$ & 19 & 22 & 0.63 & 1490 & $0(0)$ \\
a22f0n00_5 & 9867 & 0.07 & 19 & 22 & 0.73 & 9654 & $0(3)$ & 19 & 22 & 0.73 & 9654 & $0(4)$ \\
\hline a32f0n00_1 & 2011 & 0.04 & 31 & 32 & 0.52 & 2945 & $0(0)$ & 32 & 32 & 0.52 & 2944 & $0(1)$ \\
a32f0n00_5 & 16921 & 0.05 & 31 & 32 & 0.59 & 22750 & $2(10)$ & 31 & 32 & 0.59 & 22750 & $2(11)$ \\
\hline a42f0n00_1 & 2865 & 0.03 & 44 & 42 & 0.35 & 7761 & $0(2)$ & 52 & 42 & 0.37 & 7228 & $0(2)$ \\
a42f0n00_5 & 24366 & 0.04 & 44 & 42 & 0.42 & 60042 & $5(28)$ & 46 & 42 & 0.42 & 60040 & $6(29)$ \\
\hline t32f0n00_1 & 7717 & 0.03 & 30 & 33 & 0.37 & 15064 & $1(15)$ & 31 & 33 & 0.37 & 15062 & $1(12)$ \\
t32f0n00_5 & 64829 & 0.04 & 30 & 33 & 0.39 & 125429 & $9(154)$ & 30 & 33 & 0.39 & 125429 & $8(160)$ \\
\hline
\end{tabular}

(b)

Table 3.1: (a) Comparison of the precision results between the proposed approach and approach in [80] for small examples. (b) Comparison of precision results between models obtained by three discovery algorithms, for large examples where $a_{b}^{\prime}$ was not able to finish.

Larger benchmarks for which Conformance Checker cannot handle are provided in Table 3.1b. For these benchmarks, we report the results (precision value, number of $|M I L|$ and CPU time in seconds) for the models obtained by the ILP miner and the RBMiner [90]. These are two miner that guarantee fitness value one. For each one of the $a N$ benchmarks, $N$ represents the number of tasks in the log, while the $\_1$ and $\_5$ suffixes denote its size: 100 and 900 traces, respectively. The $t 32$ has $200\left(\_1\right)$ and 1800 (_5) traces. The pair of CPU times reported denote the computation of $e t c_{p}$ without or with the collection of $|M I L|$ (in parenthesis). Also, we provide the results of the most permissive models, i.e., models with only the transitions but without arcs or places $\left(M_{T}\right)$. These models allow any behavior and thus, they have a low etc $c_{p}$ value, as expected. 
A first conclusion on Table 3.1 (b) is the capability of handling large benchmarks in reasonable CPU time, even for the prototype implementation carried out. A second conclusion is the loss of precision of the metric with respect to the increase of abstraction in the mined models: as soon as the number of tasks increases, the miners tend to derive models less precise to account for the complex relations between different tasks. Often, these miners derive models with a high degree of concurrency, thus accepting a potentially exponential number of sequences which might not correspond to the real number of traces in the log.

Finally, three charts are provided: the relation between the log size with respect to the CPU time, the $e t c_{p}$ value and the size of $M I L$ are shown in Fig. 3.10. For these charts, we selected different log sizes for different types of benchmarks (a22f0, a22f5, a32f0,a32f5 for the two bottom charts, a42f0, t32f5 and t32f9 for the top chart). For the two bottom charts, we used the Petri nets derived by the ILP miner to perform the conformance analysis on each log, whereas we use a single Petri net for the top chart to evaluate the CPU time (without collecting $M I L$ ) on different logs, illustrating the linear dependence of our technique on the log size. The chart on top clearly shows the linear relation between log size and CPU time for these experiments, which is expected by the technique presented in this chapter. The two charts on bottom of the figure show: (left) since for the a22/a32 benchmarks the models derived are very similar independently of the $\log$, the more traces are included the less escaping arcs are found. On the other hand, the inclusion of more traces contributes to the incorporation of more traces in the $M I L$, as it is shown in the right chart at the bottom. 


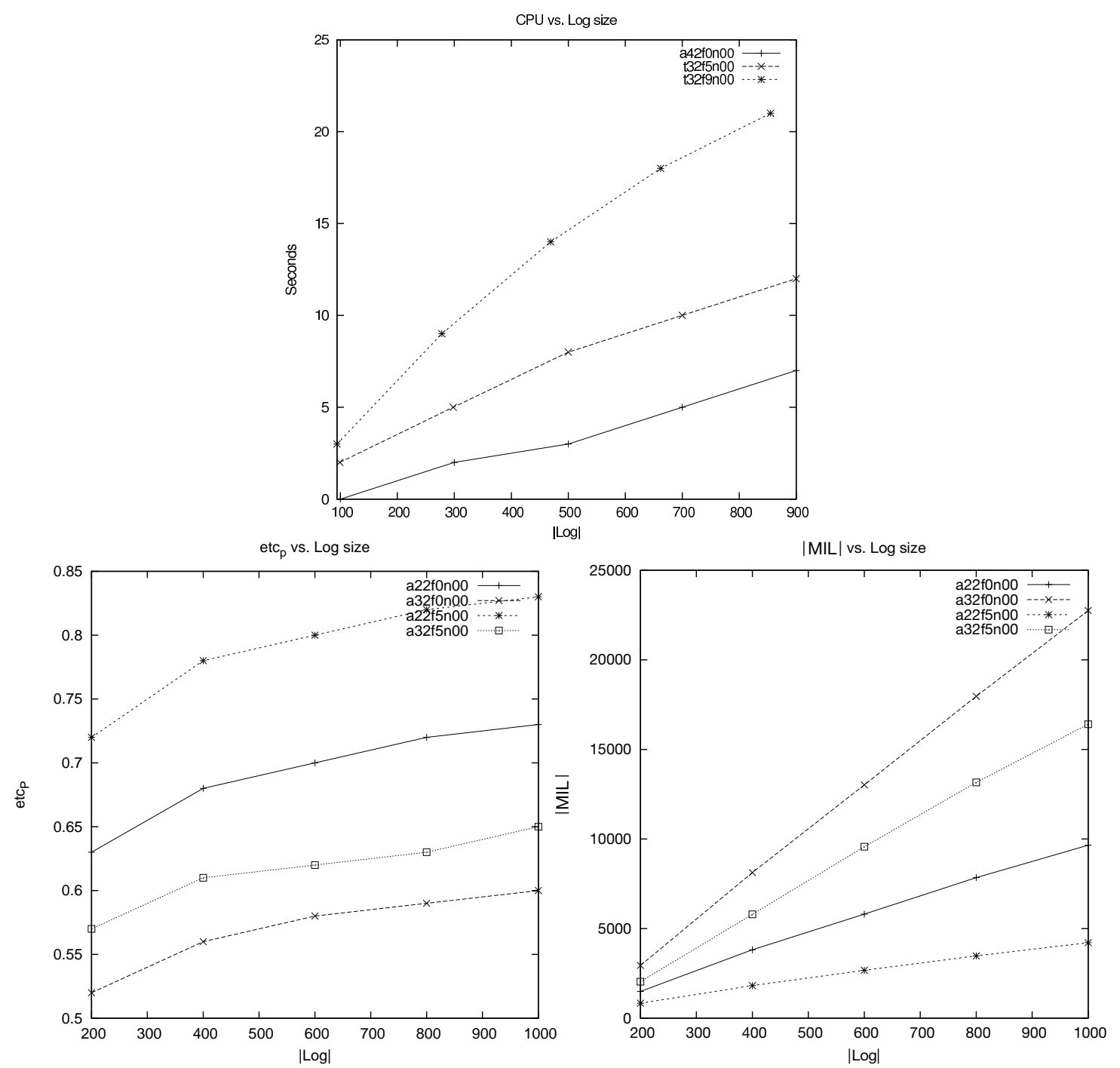

Figure 3.10: Relation between time, et $c_{p}$, minimal imprecise log, with respect to the log size for some large benchmarks. 


\subsection{Limitations and Extensions}

For the sake of clarity, the approach presented in this chapter made some assumptions. In this section we discuss the effects of relaxing those assumptions. In particular, we focus on the consequences of unfitting traces and the indeterminism between activity sequences and tasks sequences.

\subsubsection{Unfitting Scenario}

This chapter has presented the precision checking based on escaping arcs assuming a perfectly fitting $\log$. In other words, each trace on a $\log L$ is included in the set of complete activity sequences of the model $M$. Consequently, any prefix in the log traces is a prefix of the sequences of the model, and therefore, $\operatorname{observed}(\sigma) \subseteq \operatorname{modeled}(\sigma)$ for all states $\sigma \in S_{L M}$.

However, this is a strong assumption, specially in real-life scenarios, where misrecorded events and exceptional executions of the process exists. For such cases, we define $S_{L}=\{\sigma \mid \sigma \in$ - $(L) \wedge \sigma \notin \bullet(\phi(M))\}$ as the set of states in the observed behavior but not modeled. Those states represent situations difficult to interpret, where the observed behavior is not aligned within the domain of the model [15]. In other words, the model is not able to determine the state of the system given the observed elements.

Given the rare nature of these situations, a possible strategy is to consider the fitting part of the traces for the computation of the precision. In other words, given an unfitting trace $\sigma=\sigma^{\prime} \cdot\langle a\rangle \cdot \sigma^{\prime \prime}$ where $\sigma^{\prime} \in S_{L M}$ and $\sigma^{\prime} \cdot\langle a\rangle \in S_{L}$, only $\sigma^{\prime}$ is used to compute precision. For example, let us consider the model $M$ of Figure 3.11 and the log $L=\left[\langle a, b, d, f\rangle^{100},\langle a, c, e, f\rangle^{100},\langle a, b, c, d, f\rangle\right]$. The observed behavior used to compute the precision automaton is composed by the two first traces (100 instances of each) and the fragment $\langle a, b\rangle$ of the third trace.

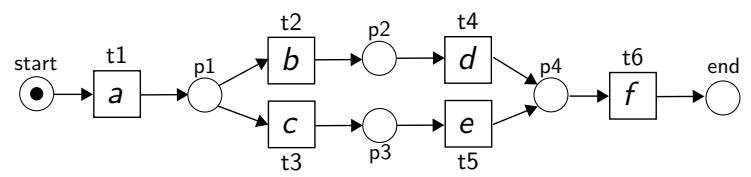

Figure 3.11: Model to illustrate the problems of unfitting scenarios.

Notice that the current definition of observed, modeled and escaping arcs presented in Definition 3.4 and 3.5 already satisfies that assumption, considering observed arcs those arcs in the behavior of both the log and the model. Moreover, the ETC Precision proposed in Definition 3.6, based on observed and escaping arcs, also satisfies the assumption. If the escaping arcs suggest situations with precision problems, we define the equivalent fitness escaping arcs as the exact points where the observed behavior deviates from the model. In these cases, the fitness escaping arcs do not provide information about the precision dimension, but simple information about the fitness of the system. Moreover, similar to the minimal imprecise trace 
(cf. Definition 3.7), we define the minimal unfitting trace as the trace fragment reaching that fitness escaping arcs. We define the minimal unfitting $\log$ as the collection of those unfitting traces in terms of a log. For example, $L=\left[\langle a, b, d, f\rangle^{100},\langle a, c, e, f\rangle^{100},\langle a, b, c, d, f\rangle\right]$ has only one fitness escaping arc, and therefore the minimal unfitting log is composed only by the trace $\langle a, b, c\rangle$.

The strategy of considering the fitting part of the traces to compute precision is only appropriate for those cases where the unfitting behavior represent a minor part of the whole observed behavior and its effect on the metric is negligible. However, it has several disadvantages and limitations. First, the precision metric is affected by the position of the fitness problem, creating a dependency between both dimensions not desired [19, 18]. In the worst case scenario, when the fitness mismatch is at the beginning of the trace, the whole trace is discarded. For example, $L_{1}=[\langle a, a, b, d, f\rangle,\langle a, a, c, e, f\rangle]$ and $L_{2}=[\langle a, b, d, f, f\rangle,\langle a, c, e, f, f\rangle]$ are two logs for the model $M$ where $a$ or $f$ is recorded twice by mistake. In $L_{1}$, only $\langle a\rangle$ is considered to measure precision (the rest of both traces is discarded). This results in a low precision value of 0.3 . On the other hand, in $L_{2},\langle a, b, d, f\rangle$ and $\langle a, c, e, f\rangle$ are considered (only the last $f$ is discarded), and this result in a perfect precision of 1 .

The second limitation is that noisy unfitting behavior distort the precision analysis, "covering" arcs that otherwise would be considered escaping. For example, let us consider the $\log L=\left[\langle a, b, d, f\rangle^{1000},\langle a, c, b, d, f\rangle\right]$. In this $\log$, the second trace, that could be considered noisy, covers the escaping arc $\langle a, c\rangle$, denoting that the lower path of the model is never used. Notice that the effect on the metric from a escaping arc (i.e., $c$ ) in a state $\langle a\rangle$ with a weight of 1000 is much more than the effect of the escaping $\operatorname{arc} e$ in a state $\langle a, c\rangle$ with a weight of 1 .

This issue is related with the third limitation: the fitness anomalies are not addressed globally, but locally. For example, given the trace $\langle a, c, b, d, f\rangle$ in the aforementioned example, we assume that deviation is produced after $\langle a, c\rangle$. However, a global vision would consider a more logical interpretation where $c$ is already a deviation, denoting a misrecorded event on a perfectly fitting trace $\langle a, b, d, f\rangle$. The application of global alignment of observed and modeled behavior for precision checking will address these issues, and is presented in detail in Chapter 5.

\subsubsection{Indeterministic Scenario}

The second assumption made for the sake of clarity during this chapter is the deterministic relation between activity sequences and tasks sequences of a model. In other words, given a sequence of activities, there is only one sequence of tasks associated with it. However, this is not always the case. Let us consider the model in Figure 3.12. Given the activity sequence $\langle a, b\rangle$, there are two transition sequences resulting in that sequence: $\left\langle t_{1}, t_{2}\right\rangle$ and $\left\langle t_{1}, t_{3}\right\rangle$. A similar situation may occur when the model contains invisible tasks.

There are different strategies to deal with indeterminism. One option is to consider and explore all possible scenarios. However, this solution could lead to a state-space explosion, making it only suitable for small and simple cases [80]. Other options include the use of 


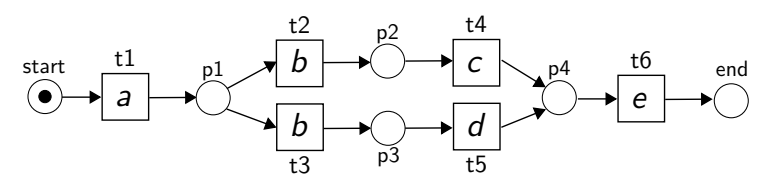

Figure 3.12: Model to illustrate the problems of indeterministic scenarios.

heuristics, e.g., a random selection, or more elaborate look-ahead heuristics to determine the most plausible option. The heuristic solution contrasts with the possibility to use alignment algorithms to determine the global optimal sequence of tasks for a given activity sequence. This possibility is explored in detail in the Chapter 5.

\subsection{Conclusions}

Given an event log and a process model, the notion of escaping arcs offers an efficient alternative to analyze the precision dimension. This chapter described the definition of the observed behavior in terms of an automaton, and how that automaton is enhanced to incorporate information about the modeled behavior. It showed how the enhanced automaton is used to detect the escaping arcs. The escaping arcs, and their position within the process, are the bases for the proposed precision metric. Furthermore, the chapter proposed the collection and analysis of the escaping arcs to get insight into the imprecision causes. Finally, the chapter includes a discussion on generalizing the approach for unfitting and indeterministic scenarios. 



\section{Chapter 4}

\section{Qualitative Analysis of Precision Checking}

\subsection{Introduction}

In the previous chapter we introduced the precision checking based on escaping arcs. Given a log and a model, we use the behavior observed in the log to traverse the modeled behavior, detecting the escaping arcs and estimating the precision of a system. In other words, it allows to know how precise is our model describing the behavior observed in the log. In this chapter, we go a step further, and we extend the escaping arcs analysis to address the following points:

- Robustness on the escaping arcs. Experience has proved that most real-life logs contain noise, e.g, incorrectly logged events (for instance, due to temporary system misconfiguration), or sequence of events representing abnormal behavior [60]. Even though noise tends to represent a minor part of the observed behavior, its effect on the escaping arcs detection may become significant, i.e., causing exceptional escaping arcs to appear, or covering legitimate ones. In this chapter we revisit the escaping arcs approach in order to make it more robust to such situations.

- Confidence of the precision metric. A checking based on escaping arcs reports the degree of precision of the system at a certain moment. However, the metric by itself provides no insight on the precision stability, i.e., how likely is the metric to drastically change when we consider more observed behavior. In this chapter we introduce a

\footnotetext{
- The materials reported in this chapter are partially published in [64] and [63]-
} 
confidence interval over the precision metric in order to indicate the possible variability of the metric in the future.

- Severity of the imprecisions. The precision based on escaping arcs aims to estimate the effort needed to achieve a perfectly precise system, i.e., resolving all the escaping arcs result in a model that precisely describes the observed behavior. However, analyzing the cause of an escaping arc and eventually fixing it requires time and it is a resourceconsuming task. One only wants to invest time and effort in resolving those deviations that represent a clear and sever precision problem. On the other hand, it may be considered a waste of resources to fix escaping arcs likely to disappear in a near future. In this chapter we propose a multi-factor severity assessment of the escaping arcs, making it possible to compare them and prioritize those imprecisions that need to be resolved first.

The remainder of this chapter is organized as follows. Section 4.2 revisits the theory of precision checking based on escaping arcs presented in Chapter 3 to introduce the notion of robustness on it. In Section 4.3 we present an upper bound and a lower bound of the precision metric to estimate its degree of confidence. Section 4.4 proposes a multi-factor analysis of the escaping arcs detected in order to estimate its severity. In Section 4.5 we provide some experimental results. Finally, Section 4.6 concludes the chapter.

\subsection{Robustness on the Precision}

The precision checking presented in Chapter 3 is based on detecting escaping arcs, i.e., the $\log$ is traversed to detect those points where the modeled behavior is broader than the one observed on the log. Each trace considered produces new escaping arcs, or covers some existing one. However, although the weight of the states is taken into account to estimate the precision metric (cf. Definition 3.6), it is not used to determine what represents or not an escaping arc. In other words, infrequent or noise behavior in the log may impact on the escaping arcs and the precision metric, covering possible escaping arcs and creating new ones.

Let us consider the insurance process in Chapter 3 and use it as a running example to illustrate the concepts introduced in this chapter. The system net of Figure 4.1 represents a model $M$ for the insurance process, and the $\log L$ in Table 4.1 is a reflect of the process execution. Each row of the log represents a trace and the number of occurrences of the trace in the log. Therefore, $L$ captures 3200 executions of the process, following 5 different paths.

Figure 4.2 shows the prefix automaton of $L$ and $M$ as it is presented in Chapter 3 . Looking at the prefix automaton one can see the effect of the infrequent trace $\sigma_{5}=\langle a, c, d, e, e, f, h, i\rangle$. The detected escaping arc $\langle a, c, d, e, e, e\rangle$ is a direct result of the trace $\sigma_{5}$, i.e., an escaping arc that would not exist if we consider the $\log L^{\prime}=\left[\sigma_{1}^{1435}, \sigma_{2}^{946}, \sigma_{3}^{764}, \sigma_{4}^{54}\right]$. On the other hand, $\sigma_{5}$ contains the prefix $\langle a, c, d, e, e\rangle$ that otherwise, considering $L^{\prime}$, would be a escaping arc on 


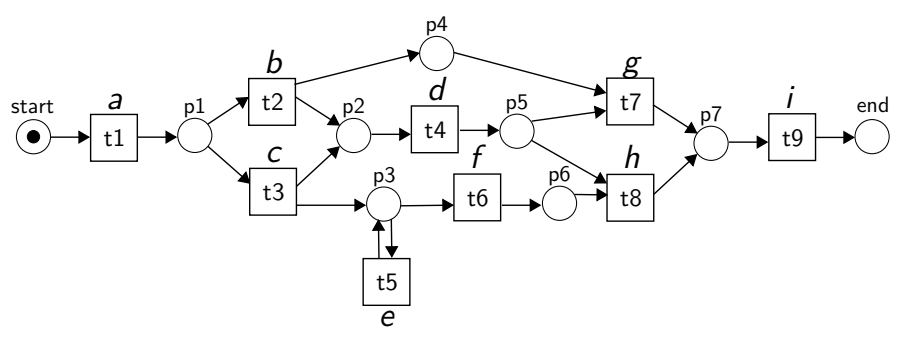

$\mathrm{a}=$ Start Checkpoint
$\mathrm{b}=$ Register Low-value Claim
$\mathrm{c}=$ Register High-value Claim
$\mathrm{d}=$ Check Policy
$\mathrm{e}=$ Consult Expert
$\mathrm{f}=$ Check Liability
$\mathrm{g}=$ Complete Low-value Claim
$\mathrm{h}=$ Complete High-value Claim
$\mathrm{i}=$ Close Checkpoint

Figure 4.1: Model $M$ for the insurance process, used as running example to illustrate the concepts of this chapter.

\begin{tabular}{cl}
\hline Frequency & Trace \\
\hline 1435 & $\sigma_{1}=\langle a, b, d, g, i\rangle$ \\
\hline 946 & $\sigma_{2}=\langle a, c, d, e, f, h, i\rangle$ \\
\hline 764 & $\sigma_{3}=\langle a, c, e, d, f, h, i\rangle$ \\
\hline 54 & $\sigma_{4}=\langle a, c, e, f, d, h, i\rangle$ \\
\hline 1 & $\sigma_{5}=\langle a, c, d, e, e, f, h, i\rangle$ \\
\hline
\end{tabular}

Table 4.1: Event $\log L$ for the insurance process, used as running example to illustrate the concepts of this chapter.

a highly weighted state $\langle a, c, d, e\rangle$ (with its consequent effect on the metric value). A robust precision checking approach should be affected as less as possible for this kind of situations.

In the literature, several approaches have been proposed to detect noisy and anomalous traces within event logs, e.g., [22, 23]. This is not the goal of the approach presented in this section, but to extend the escaping arc detection theory in order to incorporate the weight component, and to do that in an arc-level way. Therefore, the filtering of anomalous traces from the event log can be considered a pre-processing step previous to the precision checking.

In order to increase the robustness of the approach presented in Chapter 3, in this section we propose the use of a cut threshold to remove states of the prefix automaton based on their weight, as a preliminary step to the precision computation. In other words, states in the log having a weight under that threshold are considered out of the observed behavior for the precision checking purposes. For example, if we consider the state $\langle a, c, d, e\rangle$ in Figure 4.2, we see that $99.9 \%$ of its behavior follows the $f$ activity, being $e$ a promising candidate to be cut. The cut threshold proposed in this section is defined to be parametric at arc-level:

- Parametric: The cut threshold is based on a cut factor $r \in[0,1]$. The cut factor is established according to the level of robustness desired. For instance, a high cut factor 


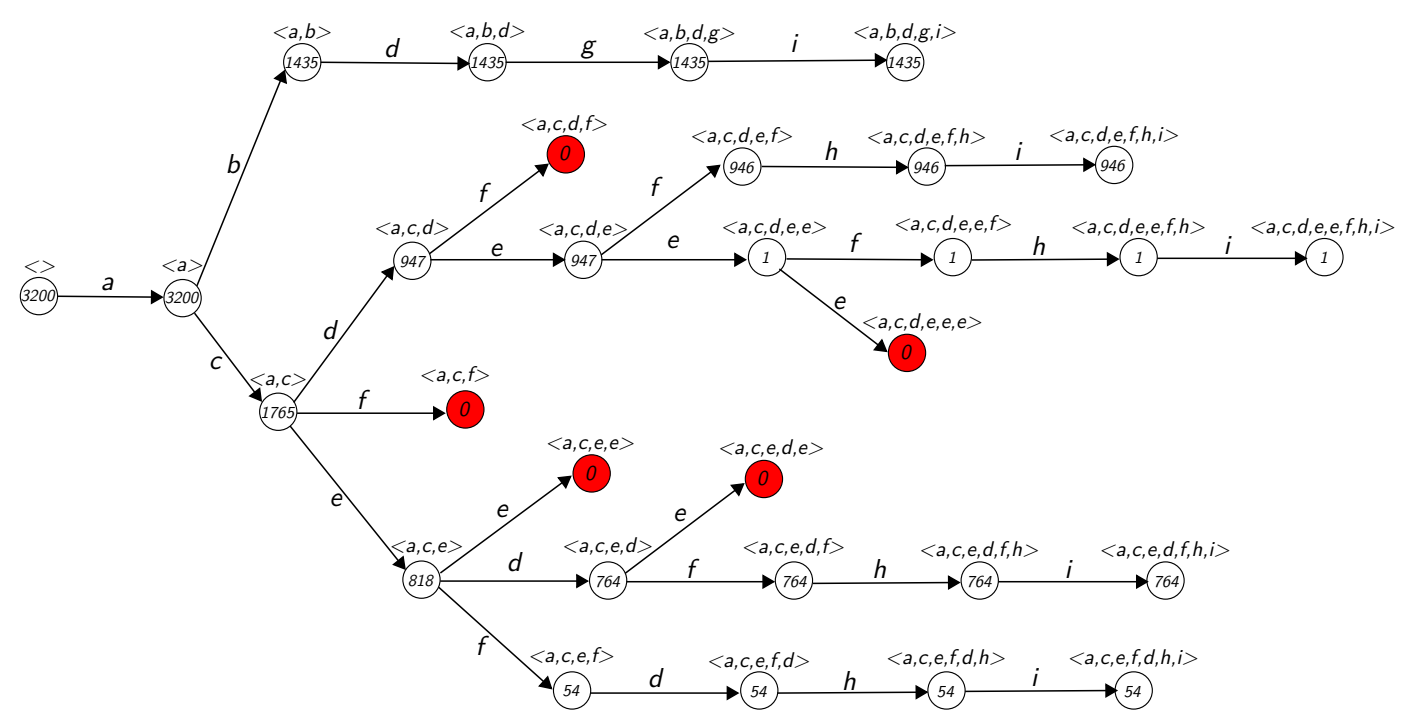

Figure 4.2: Prefix automaton for the event $\log L$ and model $M$ as it is presented in Chapter 3 .

is used to compute the precision using only the main behavior of the system, reaching a higher level of abstraction. On the other hand, a low cut factor cuts only the most extreme cases. When the cut factor $r$ is 0 there is no cut states, and all the behavior is considered for the precision checking.

- Arc-Level: The cut threshold is applied at arc-level, and not at trace level. In other words, to decide if at the state $\sigma_{t}$ with the incoming arc $q=\left(\sigma_{s}, a, \sigma_{t}\right)$ must be cut we consider only the context of that arc, i.e., the weight of target state $\sigma_{t}$ and the weight of its predecessor $\sigma_{s}$. The cut threshold is defined as the weight of the source state multiplied by the cut factor, i.e., $\omega\left(\sigma_{s}\right) \cdot r$. A state is cut if its weight is less or equal than the cut threshold, i.e., $\omega\left(\sigma_{t}\right) \leq \omega\left(\sigma_{s}\right) \cdot r$. Notice that, the cut threshold is defined locally to the arc, and not globally. Therefore, the cut does not depends on the weight of the overall automaton but only on the weights of the part of the process the arc refers to.

When a state $\sigma \in S_{L M}$ is defined as cut state, all the states in $S_{L M}$ descendants of $\sigma$ are also considered cut.

Figure 4.3 shows the prefix automaton of $L$ and $M$ when we apply a cut factor of $r=0.03$. The automaton shows some differences with the original automaton in Figure 4.2, where the cut states are represented in a different color than the escaping states. For example, the state $\langle a, c, d, e, e\rangle$ is a cut state because its weight do not overpass the threshold $\omega(\langle a, c, d, e, e\rangle) \leq$ $\omega(\langle a, c, d, e\rangle) \cdot r$, i.e., $1 \leq 947 \cdot 0.03=28.41$. Consequently, all the states after $\langle a, c, d, e, e\rangle$ 


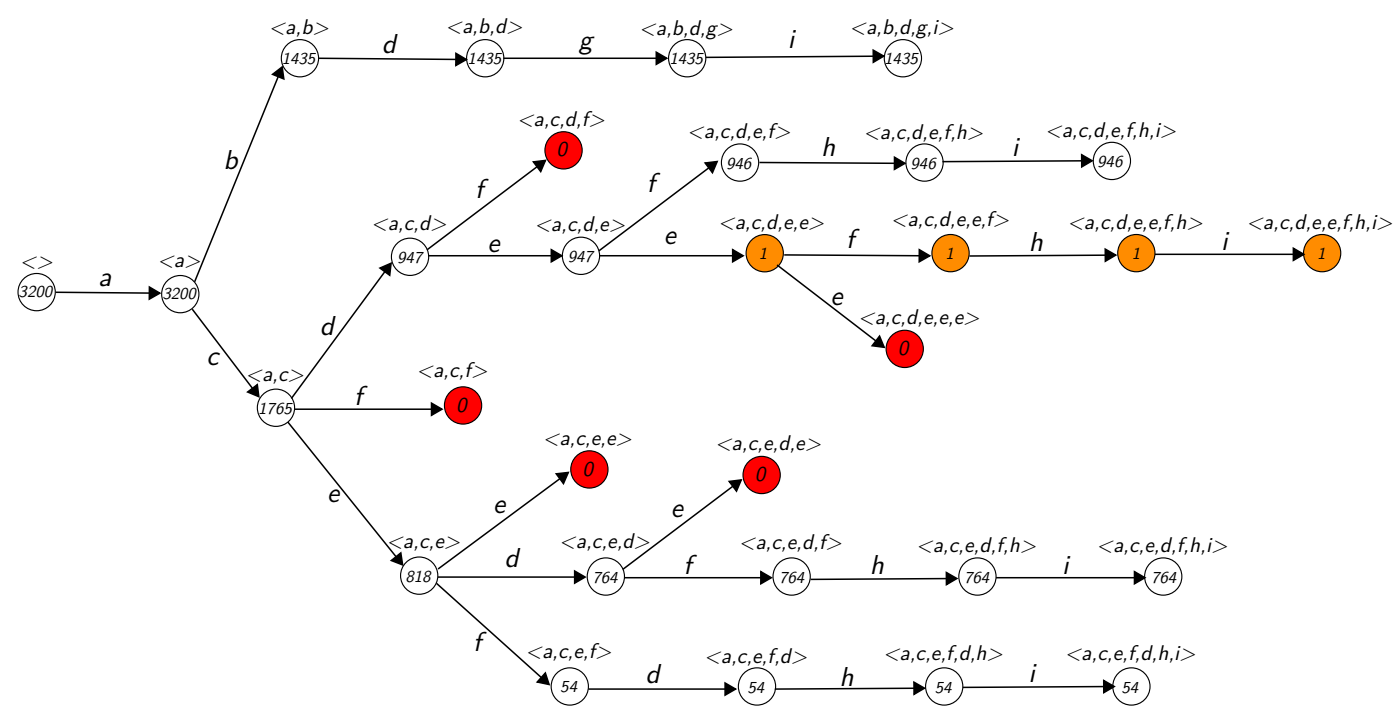

Figure 4.3: Prefix automaton for the event $\log M$ and model $M$ considering a cut factor $r=0.03$.

are also cut states, i.e., $\langle a, c, d, e, e, f\rangle,\langle a, c, d, e, e, f, h\rangle$ and $\langle a, c, d, e, e, f, h, i\rangle$.

Formally, cut states are defined as follows:

Definition 4.1 (Cut States). Let $\mathcal{A}_{L M}=(S, A, \nearrow, \omega,\langle\rangle)$ be the prefix automaton of the $\log$ $L$ enhanced with the behavior of the model $M$. Let $\left(\sigma_{s}, a, \sigma_{t}\right) \in \nearrow$ be an arc where $\sigma_{t} \in S_{L M}$ is a state of both log and model. Given a cut factor $r$, the prefix $\sigma_{t}$ is a cut state if :

- its weight is less or equal than the cut threshold for that state, i.e., $\omega\left(\sigma_{t}\right) \leq \omega\left(\sigma_{s}\right) \cdot r$.

- any of the prefixes of $\sigma_{t}$ is already a cut state, i.e., $\exists \sigma_{a} \cdot \sigma_{b}=\sigma_{t}: \sigma_{a}$ is a cut state.

The set of cut states is represented as $S_{r}$. The prefix automaton of the $\log L$ enhanced with the behavior of the model $M$ and cut with a threshold $r$ is denoted as $\mathcal{A}_{L M}^{r}$.

Notice that, by definition the empty sequence \langle\rangle is never a cut state because it has no incoming arc. Notice also that, when the threshold factor $r$ is 0 , no state in the log is cut and the approach behaves as the one presented in Chapter 3.

As it has been mentioned before, the cut states are considered to be out of the log behavior. Therefore, although they appear on the log, they are counted as escaping states for the precision computation. The definitions of modeled, observed and escaping in Definition 3.4 and Definition 3.5 are adapted to reflect this new scenario. 
Definition 4.2 (Escaping Arcs with Cut States). Let $\mathcal{A}_{L M}^{r}=(S, A, \nearrow, \omega,\langle\rangle)$ be the prefix automaton of the $\log L$ enhanced with the behavior of the model $M$ and cut with a factor $r$. Let the prefix $\sigma_{s} \in\left(S_{L M} \backslash S_{r}\right)$ represent a state of both log and model and not cut by $r$. The set of arcs observed in $\sigma_{s}$ represent those activities executed and consequently recorded on the $\log L$ but not cut by $r$, i.e., obs $\left(\sigma_{s}\right)=\left\{\left(\sigma_{s}, a, \sigma_{t}\right) \in \nearrow \mid \sigma_{t} \in\left(S_{L M} \backslash S_{r}\right)\right\}$. The set of activities modeled by the model $M$ in the state $\sigma_{s}$ is defined as $\bmod \left(\sigma_{s}\right)=\left\{\left(\sigma_{s}, a, \sigma_{t}\right) \in \nearrow \mid \sigma_{t} \in S_{M}\right\}$. The set of escaping arcs of the state $\sigma_{s}$ is determined by the difference between the arcs modeled and the arcs observed on the state, i.e., $\operatorname{esc}\left(\sigma_{s}\right)=\bmod \left(\sigma_{s}\right) \backslash \operatorname{obs}\left(\sigma_{s}\right)$.

For example, the modeled activities of the state $\langle a, c, d, e\rangle$ are $f$ and $e$, but only $f$ is considered observed, being $e$ escaping. The precision metric in Definition 3.6 is redefined to consider the new definitions of modeled and escaping arcs. The metric only explores states both in the modeled and observed behavior which are not cut.

Definition 4.3 (ETC Precision with Cut States). Let $\mathcal{A}_{L M}^{r}=(S, A, \nearrow, \omega,\langle\rangle)$ be the prefix automaton of the $\log L$ enhanced with the behavior of the model $M$ and cut with a factor $r$. The metric ETC Precision when the automaton contains cut states is defined as follows:

$$
\operatorname{etc}_{p}\left(\mathcal{A}_{L M}^{r}\right)=1-\frac{\sum_{\sigma \in\left(S_{L M} \backslash S_{r}\right)} \omega(\sigma) \cdot|\operatorname{esc}(\sigma)|}{\sum_{\sigma \in\left(S_{L M} \backslash S_{r}\right)} \omega(\sigma) \cdot|\bmod (\sigma)|}
$$

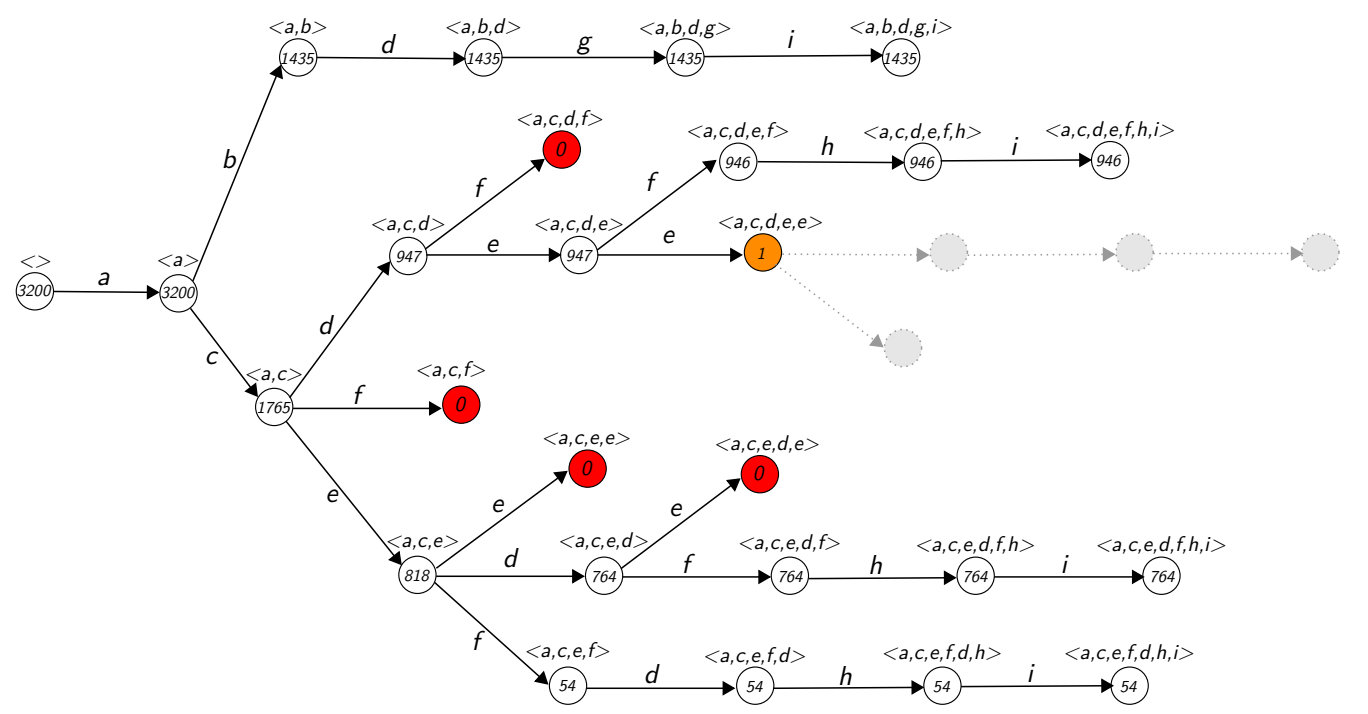

Figure 4.4: Escaping arcs of the prefix automaton for the event $\log L$ and model $M$, cut with a factor $r=0.03$. 
Figure 4.4 illustrates the states and escaping arcs considered in the metric for the running example $L$ and $M$. The five escaping states considered are represented in color: the four states in the same color indicate states in the model but not in the log, and the state in different color represents a cut state. The blurry states indicate the states not explored by the precision metric due to being cut states. is:

The metric calculation for automaton $\mathcal{A}_{L M}^{0.03}$ between $L$ and $M$ with a cut factor of 0.03

$$
\begin{aligned}
\text { etc }\left(\mathcal{A}_{L M}^{0.03}\right)=1- & \\
& \frac{3200 \cdot 0+3200 \cdot 0+1435 \cdot 0+1435 \cdot 0+1435 \cdot 0+1435 \cdot 0+\mathbf{1 7 6 5} \cdot \mathbf{1}+\ldots}{3200 \cdot 1+3200 \cdot 2+1435 \cdot 1+1435 \cdot 1+1435 \cdot 1+1435 \cdot 0+1765 \cdot 3+\ldots} \\
& \frac{\cdots+\mathbf{9 4 7} \cdot \mathbf{1}+\mathbf{9 4 7} \cdot \mathbf{1}+946 \cdot 0+946 \cdot 0+946 \cdot 0+\mathbf{8 1 8} \cdot \mathbf{1}+\mathbf{7 6 4} \cdot \mathbf{1}+\ldots}{\cdots+947 \cdot 2+947 \cdot 2+946 \cdot 1+946 \cdot 1+946 \cdot 1+818 \cdot 3+764 \cdot 2+\ldots} \\
& \frac{\cdots+764 \cdot 0+764 \cdot 0+764 \cdot 0+54 \cdot 0+54 \cdot 0+54 \cdot 0+54 \cdot 0}{} \\
& \cdots+764 \cdot 1+764 \cdot 1+764 \cdot 0+54 \cdot 1+54 \cdot 1+54 \cdot 1+54 \cdot 0 \\
= & 1-\frac{5241}{31498}=1-0.17=0.83
\end{aligned}
$$

The metric result for the automaton $\mathcal{A}_{L M}^{0.03}$ is 0.83 , and as it was expected differs from the results of the automaton $\mathcal{A}_{L M}$ with no cut where etc $_{p}\left(\mathcal{A}_{L M}\right)$ is 0.86 . The difference is explained because $\mathcal{A}_{L M}^{0.03}$ contains a escaping state $(\langle a, c, d, e, e\rangle)$ is a state with high weight $(\langle a, c, d, e\rangle$ with weight 947$)$, a situation that does not occur in $\mathcal{A}_{L M}$, causing the metric to decrease.

\subsection{Confidence on Precision}

Given an event log and a model, the metric ETC Precision $\left(e t c_{p}\right)$ estimates the degree of precision of the system. However, together with a metric, sometimes it is convenient to provide also a confidence value, indicating the possible variability of the metric in the future. In this section we provide this confidence value in terms of a confidence interval, i.e., an upper confidence value (etc $c_{p}^{\top}$ ) and a lower confidence value (etc $c_{p}^{\perp}$ ) estimating variability over the computed metric. A narrow interval indicates that the metric should not vary significantly. On the other hand, a wide interval reflects the opposite: a low confidence in the metric provided, whose value could change drastically in the future.

Both confidence values presented in this section are defined in terms of $k$, a parameter representing the future behavior to consider. A low $k$ is used to compute the confidence in a near future, whereas with a large $k$, a larger incoming behavior is considered, and thus a longer term future is contemplated. We will implement $k$ as number of traces to consider.

Notice that, both confidence values presented in this section are approximated, i.e., they do not provide real bounds over the metric, but instead aim at estimating them with simple and intuitive heuristics which can be computed in a systematic manner. 


\subsubsection{Upper Confidence Value}

Given an automaton $\mathcal{A}_{L M}^{r}$ and the parameter $k$ representing the future behavior to consider, the upper value of the confidence interval is computed considering a best possible scenario. That is, all the future behavior aims only to cover existing escaping arcs. In other words, each $j$ where $1 \leq j \leq k$ represents a new occurrence in an escaping arc, increasing the weight of the both states of the arc. In that sense, each $j$ can be seen as a future trace reaching an escaping arc $q=\left(\sigma_{s}, a, \sigma_{t}\right)$. Both if the target state $\sigma_{t}$ is not in the log or it has been cut, a trace $j$ represents a new occurrence of it in the log. If the number of occurrences is enough to overpass the cut threshold (cf. Section 4.2), the arc is no longer considered escaping.

The cost in number of occurrences needed to change a escaping arc $q=\left(\sigma_{s}, a, \sigma_{t}\right)$ from escaping to non escaping depends on the cut factor $r$ considered, i.e., $\omega\left(\sigma_{t}\right) \leq \omega\left(\sigma_{s}\right) \cdot r$. Notice that, states in the model but not in the log (i.e., with weight 0 ), are under that threshold, no matter what $r$ is considered. The technique presented in this section estimates the gain (i.e., the precision increase) of covering each escaping arc, and maximizes the total gain considering $k$ as the maximum number of occurrences used to cover escaping arcs. For that, we first define the cost and the gain of covering an escaping arc.

Definition 4.4 (Cost and Gain of Covering and imprecision). Let $q=\left(\sigma_{s}, a, \sigma_{t}\right)$ be an escaping arc of the automaton $\mathcal{A}_{L M}^{r}$. The cost of covering $q$, denoted as $\operatorname{cost}(q)=c$ with $c \in \mathbb{N}$, is the minimum $c$ that satisfies $\omega\left(\sigma_{t}\right)+c>\left(\omega\left(\sigma_{s}\right)+c\right) \cdot r$, i.e., it overpasses the cut threshold. The gain of covering the escaping arc $q$ is defined as $\operatorname{gain}(q)=\omega\left(\sigma_{s}\right)$, i.e., the gain of reducing in one the number of escaping arcs of the source state $\sigma_{s}$.

By inspecting the formula of the ETC Precision metric (cf. Definition 4.3), one can see why the gain of covering the escaping $q$ is defined as $\omega\left(\sigma_{s}\right)$ : if the state $\sigma_{s}$ has one less escaping arc, the number of escaping arcs become $\left|\operatorname{esc}\left(\sigma_{s}\right)\right|-1$. Since this number is multiplied by $\omega\left(\sigma_{s}\right)$ in the numerator part of the equation, the numerator will be reduced exactly in $\omega\left(\sigma_{s}\right)$.

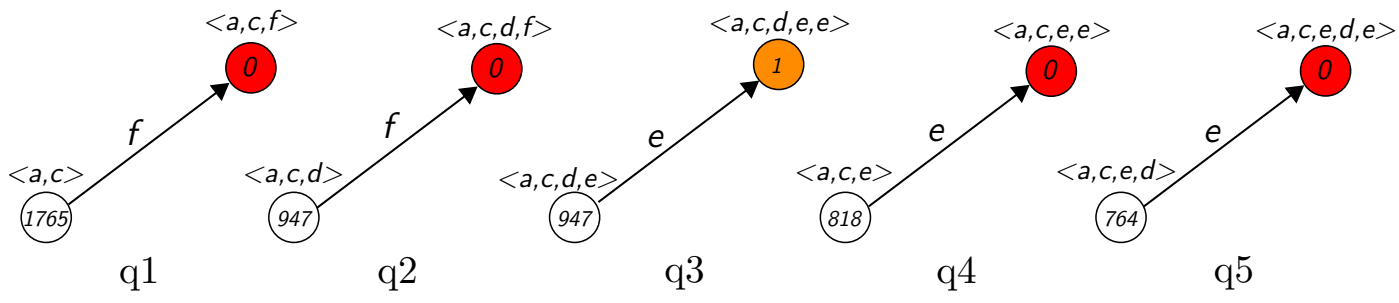

Figure 4.5: Close up of the escaping arcs of the automaton $\mathcal{A}_{L M}^{0.03}$ for the running example $M$ and $L$.

Let us consider the escaping arcs of the running example $\mathcal{A}_{L M}^{0.03}$, enumerated in Figure 4.5. The cost of the escaping arc $q_{2}$ is 30 , i.e., 30 traces need to reach that arc to overpass the 
threshold. Instead, 29 are not enough because $\omega(\langle a, c, d, f\rangle)+29 \ngtr(\omega(\langle a, c, d\rangle)+29) \cdot 0.03$. On the other hand, the escaping arc $q_{3}$ only needs 29 . That contrasts with the the cost of the escaping arc $q_{1}$ - that needs 55 - because of the weight of $\langle a, c\rangle$ - that is 1765 . The gain of covering $q_{2}$ and $q_{3}$ is the same, i.e., 947. On the other hand, the gain of covering $q_{1}$ is 1765 .

Once the gain and cost of covering an escaping arc are defined, the maximum gain obtained with $k$ occurrences can be formulated. This problem is analogous to the well known Knapsack problem [38], which can be solved using binary integer programming (BIP) [87]. The following BIP model encodes the maximum gain obtained covering imprecisions with at most $k$ occurrences:

1. Variables: The variable $X_{i}$ denotes if the imprecision $i$ is covered or not.

$$
X_{i} \in\{0,1\}
$$

2. Constraints: The total cost cannot exceed the number of occurrences.

$$
\sum_{i \in \operatorname{esc}\left(\mathcal{A}_{L M}^{r}\right)} \operatorname{cost}(i) \cdot X_{i} \leq k
$$

3. Cost function: Maximize the gain.

$$
\max \sum_{i \in \operatorname{esc}\left(\mathcal{A}_{L M}^{r}\right)} \operatorname{gain}(i) \cdot X_{i}
$$

Once the optimization problem is formulated, the upper confidence value can be defined as follows:

Definition 4.5 (Upper Confidence Value). Let $\mathcal{A}_{L M}^{r}=(S, A, \nearrow, \omega,\langle\rangle)$ be the prefix automaton of the $\log L$ enhanced with the behavior of the model $M$ and cut with a factor $r$. Let $k$ be the future parameter to consider. Let numerator and denominator be the numerator and denominator of the metric ETC Precision in Definition 4.3, i.e., etc ${ }_{p}\left(\mathcal{A}_{L M}^{r}\right)=1-\frac{\text { numerator }}{\text { denominator }}$. Let gain $_{\max }$ be the result obtained using the optimization problem modeled above. The upper confidence value is defined as:

$$
\operatorname{etc}_{p}^{\top}\left(\mathcal{A}_{L M}^{r}, k\right)=1-\frac{\text { numererator }- \text { gain }_{\max }}{\text { denominator }}
$$

Following with the running example of $\mathcal{A}_{L M}^{0.03}$ and considering a future parameter $k=24$, the only escaping arc with cost lower enough to be covered with this $k$ is $q_{5}$. The gain of covering this escaping arc is 764 . This value is subtracted from numerator, providing an upper interval value of 0.85 for this scenario. 


\subsubsection{Lower Confidence Value}

The idea for computing the lower confidence value is similar to the upper value. However, in this case the $k$ representing the future does not cover escaping arcs, but produce the rising of new escaping arcs instead. In other words, we consider $k$ new observed traces. Each one of those $k$ traces introduces $m$ new observed states. And each one of those $m$ states causes $n$ new escaping arcs to appear. The lower confidence value is the decrease on the precision metric caused by the escaping arcs introduced by these new $k$ traces.

The number of escaping arcs introduced is directly related with the number of observed states introduced by the new traces, i.e., $m$. Longer traces cause more escaping arcs, and as a result a lower confidence value. The are several alternatives of the value of $m$, e.g., the length of the longest trace in the log, or just an arbitrary number. However, for statistical consistency, the $m$ considered in this approach corresponds with the average length of the traces in the log. Moreover, the number of new escaping arcs introduced for each state (i.e., $n$ ) is also directly related with the confidence value obtained. Given that we are considering a worst case scenario, we consider $n$ to be $|A-1|$, i.e., all activities are escaping arcs except the one followed by the trace. Given all these considerations, the lower confidence value is defined as follows:

Definition 4.6 (Lower Confidence Value). Let $\mathcal{A}_{L M}^{r}=(S, A, \nearrow, \omega,\langle\rangle)$ be the prefix automaton of the $\log L$ enhanced with the behavior of the model $M$ and cut with a factor $r$. Let numerator and denominator be the numerator and denominator of the metric ETC Precision in Definition 4.3, i.e., et $c_{p}\left(\mathcal{A}_{L M}^{r}\right)=1-\frac{\text { numerator }}{\text { denominator }}$. Let $k$ be the future to consider, and let avg be the average length of the traces in the $\log L$. The lower confidence value is defined as:

$$
\operatorname{etc}_{p}^{\perp}\left(\mathcal{A}_{L M}^{r}, k\right)=1-\frac{\text { numerator }+(k \cdot \operatorname{avg} \cdot|A-1|)}{\text { denominator }+(k \cdot \operatorname{avg} \cdot|A|)}
$$

For instance, following with the running example, being avg $=6$ the average length, $A=8$ the number of activities, and considering $r=0.03$ and $k=24$, the lower bound in this case is:

$$
\operatorname{etc}_{p}^{\perp}\left(\mathcal{A}_{L M}^{r}, 24\right)=1-\frac{5241+(24 \cdot 6 \cdot 7)}{31498+(24 \cdot 6 \cdot 8)}=0.81
$$

\subsection{Severity of an Escaping Arc}

The computation of the escaping arcs is an accurate mechanism to determine the exact location of imprecisions of a system. By means of defining the border between log and model behaviors one can indicate where the efforts must be done in order to achieve a precise representation of the reality. However, not all escaping arcs have the same importance. Some escaping arcs may refer to exceptional and infrequent parts of the process or may be produced by the incompleteness of the log considered. On the other hand, other escaping 
arcs may be clear and may affect important and frequent parts of the process. Assigning to each escaping arc a severity degree it becomes possible to compare and sort the escaping arcs, opening the door to prioritizing those imprecision points that must be fixed first.

The severity of an escaping arc is a complex multi-factored concept, with a strong degree of subjectivity that changes according to the importance that each process analyst gives to each factor.

Definition 4.7 (Severity of an Escaping Arc). Let $q \in \operatorname{esc}\left(\mathcal{A}_{L M}^{r}\right)$ be an escaping arc detected in the system. The severity of $q$ is defined as:

$$
\operatorname{sev}(q)=f\left(f a c t_{1}^{q}, \ldots, f a c t_{n}^{q}\right)
$$

where $f a c t_{1}^{q}, \ldots$, fact $_{n}^{q}$ correspond to the factor values for the escaping arc $q$, and $f$ is a user-defined function that weights the $n$ factors.

In this section, we define a standard weighting function as the arithmetic mean of all the factor values considered, giving the same importance to all the factors. Alternative weighting functions can be defined assigning higher weights to some factors, and lower weights (or even 0) to others.

The definition and selection of the different factors considered in the severity function are also open to subjectivity, and may vary from one context to another. In this thesis we propose a four-factored severity, being weight, alternation, stability and criticality the four factors considered.

\subsubsection{Weight of an Escaping Arc}

The first factor proposed to assess the severity of an escaping arc is the weight. Escaping arcs occurring on highly weighted states denote problems in important parts of the process. These escaping arcs occur frequently during the process executions, and should be in the top of the list of problems to fix. On the other hand, escaping arcs that appear on states with low weight indicate problems in infrequent parts of the process, with low representation in the process executions.

The weight factor considers the weight of the state where the escaping arc occurs, normalizing it with the maximum weight of all the states in the automaton. Therefore, the factor value ranges from 0 to 1 .

Definition 4.8 (Weight Factor). Let $q=\left(\sigma_{s}, a, \sigma_{t}\right) \in \operatorname{esc}\left(\mathcal{A}_{L M}^{r}\right)$ be an escaping arc detected in the automaton $\mathcal{A}_{L M}^{r}=\left(S, A, \nearrow, \omega, \sigma_{0}\right)$. Let $\max$ be the maximum weight of all the states in $S$, i.e., $\forall \sigma \in S, \max \geq \omega(\sigma)$. The weight factor of the escaping arc $q$ is defined as:

$$
\operatorname{fact}_{w}^{q}=\frac{w\left(\sigma_{s}\right)}{\max }
$$


For example, let us consider the escaping arc $q_{1}$ of the running example $\mathcal{A}_{L M}^{0.03}$. The maximum weight of all the states of $\mathcal{A}_{L M}^{0.03}$ corresponds with the state \langle\rangle , i.e., $\max =3200$. Therefore, the weight factor for $q_{1}$ is $\operatorname{fact}_{w}^{q_{1}}=\frac{w(\langle a, c\rangle)}{\max }=\frac{1765}{3200}=0.55$. The mid-value weight factor for $q_{1}$ contrast with the $\frac{764}{3200}=0.24$ value for the imprecision $q_{5}$.

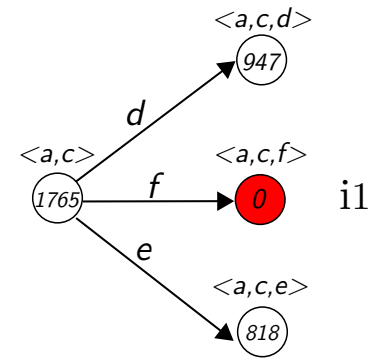

Figure 4.6: Close-up of the escaping arc $q_{1}$ of $\mathcal{A}_{L M}^{0.03}$ and it context.

\subsubsection{Alternation of an Escaping Arc}

The alternation factor addresses those situations where the system enables a set of alternatives, but only a few part of them can be considered valid alternatives. In this case, the set of alternatives are the set of arcs in a state, while a wrong alternative is to chose to continue through an escaping arc. Those situations where it is more likely to chose wrongly must have more priority, than others situations where choosing an escaping arc is not so probable.

Definition 4.9 (Alternation Factor). Let $q=\left(\sigma_{s}, a, \sigma_{t}\right) \in \operatorname{esc}\left(\mathcal{A}_{L M}^{r}\right)$ be an escaping arc detected in the automaton $\mathcal{A}_{L M}^{r}=\left(S, A, \nearrow, \omega, \sigma_{0}\right)$. Given a state $\sigma \in S$, let $P_{E}(\sigma)$ be the probability of selecting a escaping arc being in the state $\sigma$. The alternation factor of the imprecision $q$ is defined as:

$$
\text { fact }_{a}^{q}=P_{E}\left(\sigma_{s}\right)
$$

The distribution of $P_{E}\left(\sigma_{s}\right)$ depends on the assumptions taken. In the general case where no particular assumption are made, a uniform distribution is considered, i.e., given $\sigma_{s}$, all the outgoing arcs of $\sigma_{s}$ have the same probability of being reached. Considering a uniform distribution the alternation factor of the escaping $\operatorname{arc} q$ is reformulated as:

$$
\operatorname{fact}_{a}^{q}=\frac{|\operatorname{esc}(\sigma)|}{|\bmod (\sigma)|}
$$

Notice that the alternation is a factor based on the source state $\sigma_{s}$ of the escaping arc, not the target escaping state $\sigma_{t}$. Therefore, all escaping arcs with the same source state have the same alternation value. The alternation factor measures the amount of alternation in each escaping arc. For instance, the alternation value for the escaping arc $q_{1}$ is fact $_{a}^{q_{1}}=\frac{1}{3}=0.33$, 
denoting a mid-low probability of selecting a escaping state - only $\langle a, c, f\rangle$ in this case- from the state $\langle a, c\rangle$.

\subsubsection{Stability of an Escaping Arc}

The third factor proposed - the stability factor - addresses the stability or equilibrium of an escaping arc, i.e., the probability of an arc to stop being an escaping arc after applying to it a little perturbation. The idea of introducing perturbations in order to estimate some property has been used successfully in other fields, such as the measurement of community robustness [51]. In our setting, a perturbation over an escaping arc is represented by a small set of traces going across the escaping arc, modifying the weight of both source and target state, and possibly changing its status from escaping to normal arc. The number of traces used as perturbation - and represented by $z$ - are defined proportionally to the weight of the source state by means of the perturbation intensity $\tau$.

Definition 4.10 (Perturbation of an Escaping Arc). Let $q=\left(\sigma_{s}, a, \sigma_{t}\right) \in \operatorname{esc}\left(\mathcal{A}_{L M}^{r}\right)$ be an escaping arc detected in the automaton $\mathcal{A}_{L M}^{r}=\left(S, A, \nearrow, \omega, \sigma_{0}\right)$. Let $\tau \in[0,1]$ be the perturbation intensity considered. The number of traces considered as perturbation is defined as $z=\left\lceil\omega\left(\sigma_{s}\right) \cdot \tau\right\rceil$. Let $l \in \mathbb{N}$ be the smallest number such that the equation $\omega\left(\sigma_{t}\right)+l>$ $(\omega(\sigma)+z) \cdot r$ is satisfied, i.e., $l$ defines the minimum number of traces the arc $q$ must receive in order to overpass the cut threshold $r$ after considering $z$ traces, and change from escaping to normal arc.

For example, let us consider the escaping arc $q_{1}$ of $\mathcal{A}_{L M}^{0.03}$ where $r=0.03$. Considering a perturbation intensity of $\tau=0.06$, the perturbation traces are $\lceil 1765 \cdot 0.06\rceil=106$, and consequently, $l$ is $\lceil((1765+106) \cdot 0.03)-0\rceil=57$.

The stability factor is defined as the probability of an escaping arc to remain escaping after perturbing it.

Definition 4.11 (Stability Factor). Let $q=\left(\sigma_{s}, a, \sigma_{t}\right) \in \operatorname{esc}\left(\mathcal{A}_{L M}^{r}\right)$ be an escaping arc of $\mathcal{A}_{L M}^{r}=\left(S, A, \nearrow, \omega, \sigma_{0}\right)$. Let $\tau$ be the perturbation intensity, and let $z$ and $l$ be the corresponding perturbation traces and minimum, respectively. The stability factor of the escaping $\operatorname{arc} q$ is the probability of $q$ to remain escaping after considering $z$ new traces, i.e.,

$$
f a c t_{s \tau}^{q}=P_{q}^{z}(<l)=\sum_{i=0}^{l-1} P_{q}^{z}(=j)
$$

where $P_{q}^{z}(<x)$ and $P_{q}^{z}(=x)$ represent the probability that the arc $q$ receives less than $x$ (or exactly $x$ ) of the new $z$ traces considered in this point.

Let $p_{q}$ define the probability that a new trace crosses the escaping arc $q$. Let $1-p_{q}$ be the probability that the trace follows one of the other successor states of $\sigma_{s}$. According to the binomial distribution [25], the stability factor can be expressed as: 


$$
\text { fact }_{s \tau}^{q}=\sum_{i=0}^{l-1}\left(\begin{array}{c}
z \\
i
\end{array}\right)\left(p_{q}\right)^{i}\left(1-p_{q}\right)^{z-i}
$$

The formula can be understood as follows: in order to $q$ to remain escaping $i$ successes $\left(p_{q}\right)^{i}$ and $z-i$ failures $\left(1-p_{q}\right)^{z-i}$ are needed. However, the $i$ successes can occur anywhere among the $z$ traces, and there are $\left(\begin{array}{c}z \\ i\end{array}\right)$ different ways of distributing $i$ successes in a sequence of $z$ traces.

The probability $p_{q}$ may depend on the assumptions taken. Again, if no knowledge regarding the distribution of the $\log$ is assumed, a uniform distribution is considered. Therefore, if $c$ is the number of successors states of $\sigma_{s}$, the probability of each successor state is $1 / c$, and the formula can be rewritten as:

$$
f a c t_{s \tau}^{q}=\sum_{i=0}^{l-1}\left(\begin{array}{c}
z \\
i
\end{array}\right)\left(\frac{1}{c}\right)^{i}\left(1-\frac{1}{c}\right)^{z-i}
$$

In the running example $\mathcal{A}_{L M}^{0.03}$, given the escaping $q_{5}$ and considering $r=0.03$ and $\tau=$ 0.06 , the stability factor results in 0.67 , being $z=46$ and $l=25$. This factor reflects that this escaping arc has a mid-probability of disappearing in the close future. This contrasts with the stability 1 obtained from the escaping arc $q_{1}$, with the same $r$ and $\tau$ parameters, reflecting a really stable imprecision.

\subsubsection{Criticality of an Escaping Arc}

Finally, the criticality factor introduces domain knowledge into the severity assessment. Different activities constitute different levels of criticality within the process. For example, the possible consequences of CheckDateFormat action are not the same as the TransferMoney action, so neither their criticality. Escaping arcs in the model allowing to execute TransferMoney - an action never observed in the log on that state - must have a higher severity and should be analyzed before than escaping arcs allowing CheckDateFormat.

The criticality factor relies on a function crit defined externally by a domain expert, assessing the criticality of the activities of the process. If this function is not defined, we consider a function where all activities have the same criticality. The criticality function is inspired by cost function used by the alignment conformance algorithms to weight the different types of misalignments $[16,15]$.

Definition 4.12 (Criticality Factor). Let $q=\left(\sigma_{s}, a, \sigma_{t}\right) \in \operatorname{esc}\left(\mathcal{A}_{L M}^{r}\right)$ be an escaping arc detected in the automaton $\mathcal{A}_{L M}^{r}=\left(S, A, \nearrow, \omega, \sigma_{0}\right)$. Let $\mathrm{cri}: A \rightarrow[0,1]$ be the function that assess the criticality of the process activities. The criticality factor of the escaping arc $q$ is defined as:

$$
f a c t_{c}^{q}=\operatorname{crit}(a)
$$


In our running example, we consider that Check Liability $(f)$ is a relative cheap operation where the database is queried automatically, whereas Consult Expert (e) is a much costly operation that involve a human consultation. Consequently, considering $\operatorname{crit}(e)=0.9$ and $\operatorname{crit}(f)=0.2$, the escaping $\operatorname{arcs} q_{3}, q_{4}, q_{5}$ are considerably more critical than $q_{1}, q_{2}$.

\subsubsection{Visualizing the Severity}

The severity of an escaping arc is the result of combining the different factors through the function $f$, that assign weights to each factor (cf. Definition 4.7). For example, the standard severity proposed in this chapter for an escaping arc $q$ is defined as $\operatorname{sev}(q)=$ mean $\left(f a c t_{w}^{q}\right.$, fact $_{a}^{q}$, fact $\left._{s \tau}^{q}, f a c t_{c}^{q}\right)$. This value can be used to visualize the severity escaping arcs of a system in more advanced ways than simply displaying the value next to the arc. For example, the set of escaping arcs can be partitioned according to their severity $([0.0,0.3)=$ low, $[0.3,0.4)=$ mid, and $[0.4,1.0)=$ critical $)$, and using an intuitive traffic light color scheme (i.e., green, yellow, red), an analyst can easily identify visually the different severity degrees.
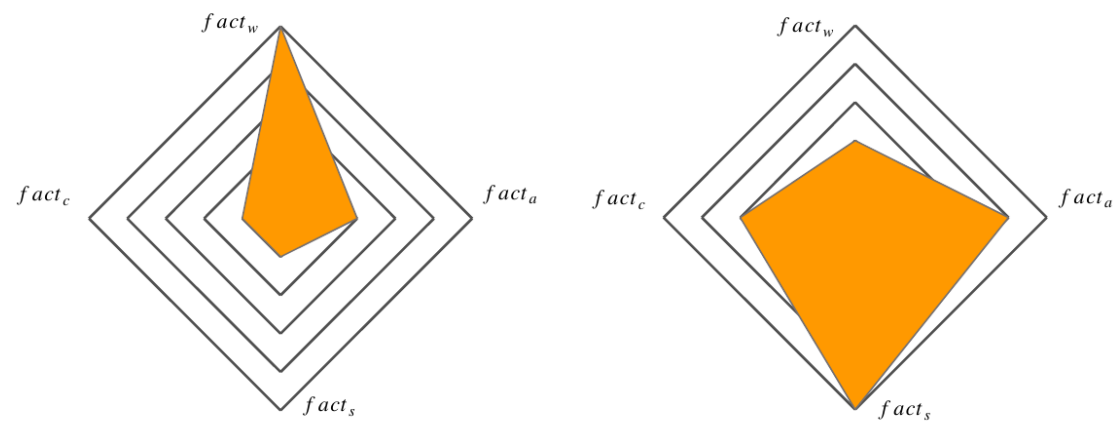

Figure 4.7: Two escaping arcs with different factor value distribution.

However, there are situations where a factor-grained analysis of the severity may be more adequate, i.e., analyzing the factors independently, instead of aggregating them on a unique value. For those cases, displaying the factor results in a radar chart provides a better understanding of the different factor distribution composing the severity of an escaping arc. For example, Figure 4.7 two possible escaping arcs with different factor distribution. The left chart corresponds to a really frequent escaping arc. However, the situation of that escaping arc is really unstable and the possibilities of choosing badly in that situation are really few. The second escaping arc, shown in the chart on the right, is much more sever in general terms than the first one. It corresponds to a more stable and dangerous situation, but it is less frequent than the first one. 


\subsection{Experimental Results}

This section illustrate experimentally some of the concepts presented on this chapter. Table 4.2 contains the results of applying the proposed approach to a set of large datasets. For comparative reasons, the datasets are some of the ones used in Chapter 3, obtained from the same public repository. As it is mentioned in Chapter 3, these datasets cannot be handled by precision approaches such as $a^{\prime} b$ [80]. The experimental setting is based on variations of the $a 32 f 0 n 00 \_5$ and $t 32 f 0 n 00 \_5$ datasets, and the experiments focus on illustrating how the growth of a log influences the metric and its confidence, given a particular selection of the stability and confidence parameters presented in this chapter. The column with $p X$ reports the percentage of the log considered in each case, i.e. p100 represents the original a32f0n00_5 $\log$, while $\operatorname{logs} p X$ with $X<100$ correspond to slices of the original log, e.g., $p 20$ contains the first $20 \%$ of the original $\log$ traces. $\operatorname{Logs} p X$ with $X>100$ are obtained by choosing with uniform distribution among the existing traces in the log the extra traces needed to achieve the desired size.

\begin{tabular}{|c|c|c|c|c|c|c|c|c|}
\hline \multicolumn{2}{|c|}{ Bench } & $|\overline{\mathbf{L o g}}|$ & $r$ & $\mathrm{k}$ & $e t c_{p}$ & \multicolumn{2}{|c|}{ Confidence } & time(s) \\
\hline \multirow{9}{*}{ a32 } & $\overline{\mathrm{p} 20}$ & 180 & \multirow{9}{*}{.05} & \multirow{9}{*}{20} & 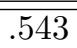 & $7.246-.553$ & $\overline{(.307)}$ & $1 / 3 / 5$ \\
\hline & $\mathrm{p} 40$ & 360 & & & .564 & $.345-.570$ & $(.225)$ & $1 / 5 / 6$ \\
\hline & p60 & 540 & & & .576 & $.403-.582$ & $(.179)$ & $1 / 7 / 11$ \\
\hline & p80 & 720 & & & .583 & $.441-.587$ & $(.146)$ & $1 / 12 / 17$ \\
\hline & p100 & 900 & & & .592 & $.470-.595$ & $(.125)$ & $1 / 15 / 24$ \\
\hline & $\mathrm{p} 150$ & 1350 & & & .591 & $.504-.595$ & $(.091)$ & $2 / 16 / 23$ \\
\hline & p200 & 1800 & & & .591 & $.523-.595$ & $(.072)$ & $2 / 17 / 23$ \\
\hline & p250 & 2250 & & & .590 & $.534-.594$ & $(.060)$ & $2 / 16 / 24$ \\
\hline & p300 & 2700 & & & .591 & $.544-.594$ & $(.050)$ & $2 / 16 / 24$ \\
\hline \multirow{9}{*}{$\mathrm{t} 32$} & p20 & 360 & \multirow{9}{*}{.05} & \multirow{9}{*}{20} & .385 & $.250-.387$ & $(.137)$ & $2 / 67$ / 121 \\
\hline & $\mathrm{p} 40$ & 720 & & & .391 & $.305-.392$ & $(.087)$ & $4 / 180 / 229$ \\
\hline & p60 & 1080 & & & .392 & $.330-.393$ & $(.063)$ & $5 / 295 / 339$ \\
\hline & p80 & 1440 & & & .393 & $.345-.394$ & $(.049)$ & $6 / 336 / 496$ \\
\hline & p100 & 1800 & & & .393 & $.353-.394$ & $(.041)$ & $6 / 390 / 550$ \\
\hline & p150 & 2700 & & & .393 & $.365-.393$ & $(.028)$ & $6 / 411 / 562$ \\
\hline & p200 & 3600 & & & .393 & $.371-.393$ & $(.022)$ & $7 / 429 / 572$ \\
\hline & p250 & 4500 & & & .393 & $.376-.393$ & $(.017)$ & $9 / 440 / 579$ \\
\hline & p300 & 5400 & & & .393 & $.379-.393$ & $(.014)$ & $9 / 443 / 581$ \\
\hline
\end{tabular}

Table 4.2: Precision metric value, confidence and computation time for incremental benchmarks.

As in the previous experiment in Chapter 3, the models used are the ones obtained from discovering a Petri net through the ILPMiner [101]. The wide spectrum of the set of 
benchmarks presented makes it possible to illustrate the evolution of the approach presented in this chapter and can be considered as a real situation in an information system where trace sets are evaluated on a regular basis, e.g., monthly.

A first conclusion on the table is the stability of the approach with respect to the size of the log. Notice that the $e t c_{p}$ value tends to increase as new behavior is considered, e.g., between p20 and p100 there is a difference of 0.05 . However, this difference is extremely small considering that between $p 20$ and $p 100$ there is a $500 \%$ increment in the observed behavior. In addition, the more traces are included in the previously observed behavior, the closer the metric value is to stabilizing. The second conclusion to extract from this table is the dependency between the traces considered and the confidence in the metric, i.e., increasing the size of the trace set considered results in a narrower confidence interval.

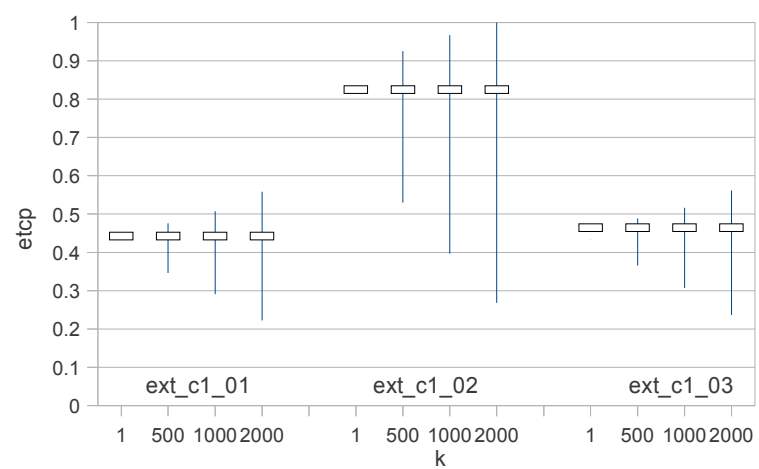

(a)

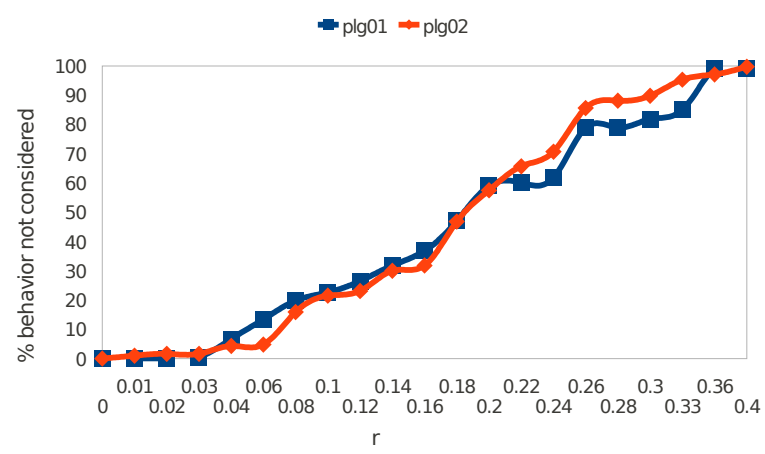

(b)

Figure 4.8: Charts showing the effects of different parameters for the confidence value. 
The next set of experiments are designed to illustrate the influence of confidence parameter $k$ and the cutting parameter $r$ in the proposed approach.

In chart $4.8 \mathrm{a}$, three process models are considered: ext_c1_01, ext_c1_02 and ext_c1_03. These benchmarks have been created using the PLG tool [36]. This tool allows to create configurable and generic benchmarks, containing all the common patters appearing in any workflow model, e.g., choice, parallelism, sequence, etc. For the experiment, each one of the logs considered contains 15000 traces. Benchmarks 01 and 03 denote standard processes, with great difference between model and log behavior (and thus exhibiting low precision). On the other hand, process model ext_c1_02 is a simpler model which describes accurately the behavior reflected in the log, i.e., the precision value is high. The chart illustrates the influence in the approach when considering diverse future windows, i.e., four different $k$ values: 1, 500, 1000 or 2000 new traces to consider. As it is reflected in the experiments, the future considered has no influence on the metric value, but it is relevant on the confidence value over the metric. The possibility of variation for the metric considering a really near future (i.e. $k=1$ ) is practically zero. However, when considering farther futures, this possibility increases, e.g., considering a $k$ value of 2000 (approx. 15\% of the log) the confidence in the metric is substantially low. Notice that, as expected, the confidence interval is not symmetric.

Chart 4.8b illustrates the relation between the cut factor $r$ and the percentage of the process behavior considered to compute the precision. Two generic and representative process, plg_01 and plg_02, have been created using PLG tool, and different values of $r$ have been tested. The conclusion we obtain is that for these processes, lower values of $r$ (i.e., less than 0.04) can be used to polish the effects produced by noisy traces, while greater values of $r$ (not considering more than $10 \%$ of the process behavior for computing the precision) should be used if the emphasis is in computing precision on the most frequent parts of the process. Values greater than 0.4 does not make any sense, due to the $100 \%$ of process is discarded.

In addition, charts $4.9 \mathrm{a}$ and $4.9 \mathrm{~b}$ are used to illustrate the part of the approach concerning the severity. Four generic models (sev01, sev02, sev03 and sev04) have been created using the PLG tool, which contain the most common structures in workflow models. For each model, a severity analysis has been performed, where each factor has received the same weight.

The same values of $r$ and $\tau$ have also been assigned for all the models $(0.01$ and 0.06 , respectively). The criticality value assigned to each of the tasks is different depending on the task and the model. In chart $4.9 \mathrm{a}$, we selected the most sever escaping arc of each process, and show the distribution of each one of the four factors. This chart illustrates the distribution of the weight of each factor in the final severity value, in this particular setting. In addition, it also illustrates that, given the normalization introduced in the definition of each one of the factors, it is possible to compare the severity between imprecision of different processes, e.g., given a system containing the four process models, and given the current setting, the escaping arc shown in the second bar (which corresponds to model sev02) should be tackled first. In chart $4.9 \mathrm{~b}$, the same four processes are considered. In this case, escaping arcs of each process are classified in three categories: low (less than 0.3), mid (between 0.3 


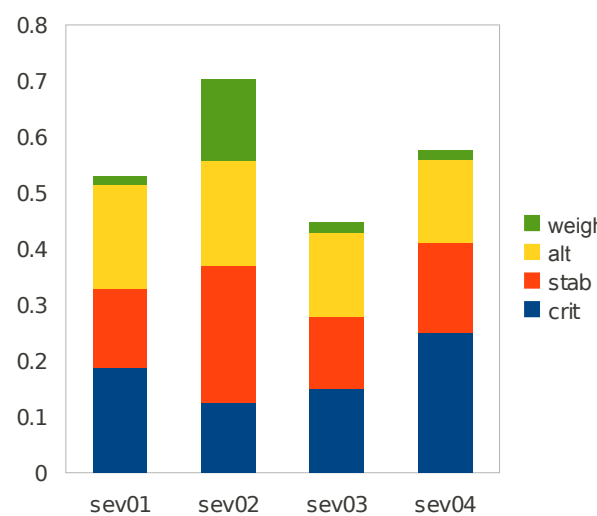

(a)

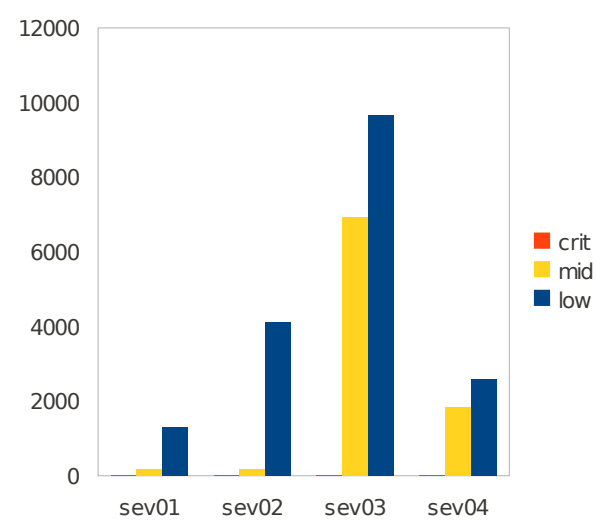

(b)

Figure 4.9: Severity analysis of four escaping arcs, and partitioning of the detected escaping arcs in three severity categories: low, mid, and critical.

and 0.4) and critical (greater than 0.4). Notice that, in this particular scenario, the number of critical escaping arcs (the ones that should be tackled urgently) is small (approx. 10 imprecisions for each process) compared to the total number of escaping arcs.

Finally, the approach presented in this chapter has been tested in a real world scenario. The scenario is taken from a Dutch Academic Hospital, and the log contains about 150.000 events in 1100 cases, where each case corresponds with a patient of the Gynecology department. ${ }^{1}$ The goal of the experiment is to measure the quality of the models obtained using different discovery algorithms. The process miners used in the experiment are RBMiner [90], Genet [37], ILPMiner [101] and $\alpha$-miner [13]. The results illustrate that the precision of the models obtained using such miners focusing on the whole process is very low. The models generated allow almost all the tasks most of the time, decreasing drastically the precision and consequently the quality of the models. For instance, the etc $c_{p}$ value of the models generated by $\alpha$-miner and RBMiner are 0.03 and 0.01 respectively. However, that precision increases when we apply partition and clustering techniques over the log, to focus the mining on specific parts of the process. For instance, mining a model projecting the process over the set of the 10 most frequent events will result in a precision of $0.397,0.386$, and 0.386 for Genet, ILPMiner and $\alpha$-miner respectively. In the case of RBMiner, the precision is slightly greater, i.e., 0.423 .

\footnotetext{
${ }^{1} \log$ file DOI is doi:10.4121/uuid:d9769f3d-0ab0-4fb8-803b-0d1120ffcf54 and can be found in the 3TU Datacenter (http://data.3tu.nl).
} 


\subsection{Conclusions}

Given an event log and a process model, the approach to measure precision based on escaping arcs presented in Chapter 3 is sensitive to the presence of infrequent behavior. This chapter presented an extension of the escaping arc theory to increase its robustness, by discarding exceptional behavior from the precision computation. Furthermore, the chapter presented a confidence interval over the precision metric, indicating how likely is the metric to drastically change when more observed behavior is considered. Finally, the chapter proposed a multifactor measure to quantify the severity of the detected escaping arcs, making it possible to compare them and to prioritize those imprecisions that need to be resolved first. 


\section{Chapter 5}

\section{Precision based on Alignments}

\section{$5.1 \quad$ Introduction}

As it has been illustrated in previous chapters, replaying observed behavior over the modeled behavior is an effective and efficient way to detect escaping arcs, and with them, detect potential imprecise points to be fixed. However, there are situations where the observed behavior cannot be replayed on the modeled behavior, i.e., the model cannot mimic the "moves" observed in the log. These problems are produced by the presence of unfitting traces, or undeterministic situations on the model. For example, let us consider the model $M_{1}$ at the top of Figure 5.1, and the state $\sigma_{1}=\langle a, b, c\rangle$ observed in the log. Given the choice between $b$ and $c$ in $M_{1}$, the state $\sigma_{1}$ cannot be reached by the model. A similar situation occurs for the models $M_{2}$ and $M_{3}$, in the middle and bottom of Figure 5.1, respectively. However, in this case the problems are not produced due the unreachability of the observed state, but because there is a non-bijective relation between activity sequences and tasks sequences on the model - due invisible and duplicate activities. Let us consider the model $M_{2}$ and the observed state $\sigma_{2}=\langle a, b\rangle$. What are the set of modeled activities for the state $\sigma_{2}$ ? $c$, if we consider the upper $b$ ? Or $d$, if we consider the lower $b$ ?. The use of duplicate activities may introduce - not always - undeterminitic situations such as the one illustrated. A similar situation happens with the presence of invisible activities. For example, let us consider the model $M_{3}$ and the observed state $\sigma_{3}=\langle a\rangle$. Because invisible activities are not reflected in the log, there is no way to determine if the set of modeled activities in $\sigma_{3}$ are $b$, $c$, or the empty set.

The effect of problems derived from unfitting logs can be alleviated making some assumptions over the observed behavior. For example, in Section 3.10.1 the unfitting part of the

\footnotetext{
- The materials reported in this chapter are partially published in [18] and [19] -
} 


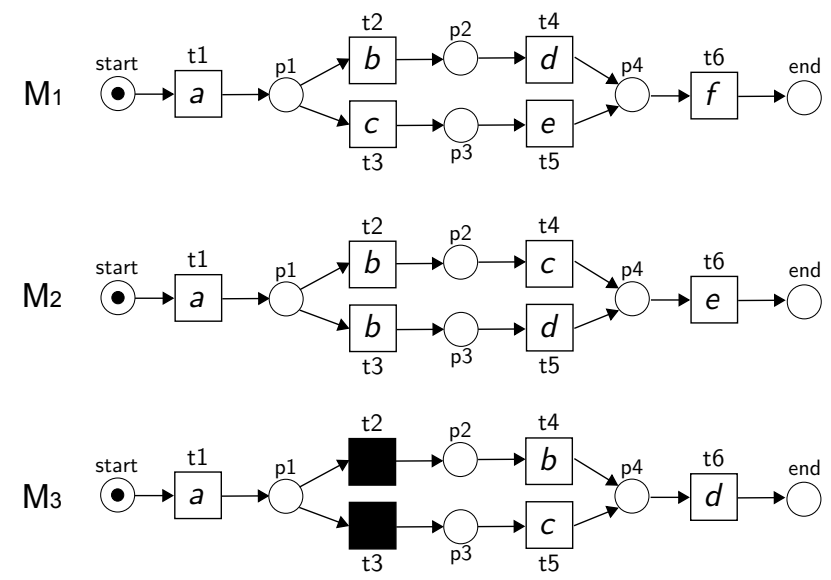

Figure 5.1: Models to illustrate the problems with the replay produced by unfitting traces (top), the indeterminism of duplicate activities (middle), and the indeterminism of invisible activities (bottom).

trace is considered noisy or infrequent and it is discarded for the precision metric. This drastic approach is useful when the percentage of log discarded is small, and we are not interested on providing all possible points of imprecision. However, the effect in a worst case scenario is not negligible. For example, in a log where only the first event in all traces is wrongly recorded, the whole log is discarded. Similar, heuristic assumptions could be considered to solve indeterministic situations with no formal guarantees, but still practical applicability.

Undeterminism is produced because the escaping arcs are detected at a log level. At a model level, each task is unique, e.g., although $t_{2}$ and $t_{3}$ have the same label $b$, they are distinguishable in the model $M_{2}$. Therefore, a precision checking at a task level will solve indeterministic situations, transferring the responsibility to mapping correctly log traces and tasks sequences of the model. Thus, each trace in the log is translated to a complete tasks sequence in the model. For example, $\log$ traces $\langle a, b, c, e\rangle$ and $\langle a, b, d, e\rangle$ may be associated with $\left\langle t_{1}, t_{2}, t_{4}, t_{6}\right\rangle$ and $\left\langle t_{1}, t_{3}, t_{5}, t_{6}\right\rangle$ of model $M_{2}$, respectively.

The mapping between log traces and model traces is far from trivial. An approach may consider the use of heuristics, such as look-ahead $[98,30]$. Informally, when the approach reaches a decision point, the algorithm looks ahead to choose the most suitable option. For example, in the trace $\langle a, b, c, e\rangle$ for the $M_{2}, t_{2}$ is selected because there is a $c$ next. Similar happens when the indeterminism is introduced by invisibles, e.g., trace $\langle a, b, d\rangle$ is associated with complete tasks sequence $\left\langle t_{1}, t_{2}, t_{4}, t_{6}\right\rangle$ in $M_{3}$. Look-ahead heuristics can be also used to associate unfitting observed stated to reachable states of the model. For example, given the observed trace $\langle a, b, c, d, f\rangle$ and the model $M_{1}$, the algorithm could consider $c$ an event wrongly recorded in the log. 
Look-ahead and other heuristics are heuristics after all, and therefore, they lack of formal guarantees. The decision taken heuristically may not be the optimal. Even, when the number of events considered in the look-ahead is increased, the decision may still not be optimal, e.g., the optimal path may require reconsidered previous decision [15].

In this chapter we introduce a precision checking approach based on aligning observed and modeled behavior. The alignment is done at a model level, for each one of the traces in the log. The alignment techniques provide global optimal results and therefore, there are guarantees on the escaping arcs detected. Notice that, the computation cost of aligning observed and modeled behavior in a global optimal may be considerable in some cases. Therefore, there are situations where other alternatives need to be considered, for example, a decomposed aligning for conformance diagnosis (cf. Chapter 6), or a heuristic replay-based approach in real-time scenarios where the time is a crucial element (cf. Chapter 9).

The remainder of this chapter is organized as follows. Section 5.2 formalizes the alignment of observed and modeled behavior. Section 5.3 proposes an approach to measure precision from the alignments. In Section 5.4, we propose two extensions for abstracting the precision checking from the order of the tasks (Section 5.4.1) and the direction of the automaton construction (Section 5.4.2). Finally, Section 5.5 provides experimental results, and Section 5.6 concludes the chapter.

\subsection{Cost-Optimal Alignment}

An alignment between an event log and a process model relates occurrences of activities in the $\log$ to tasks of the model. As the execution of a case is often performed independently of the execution of another case, the alignment is performed per traces. This is a common assumption take in process mining techniques, and reduces the complexity of the analysis.

For each trace in an event log that fits a process model, each move in the trace (i.e., an activity observed in the $\log$ ) can be mimicked by a move in the model (i.e., a task executed in the model). However, this is not the case if the trace does not fit the model perfectly. We use the symbol $\gg$ to denote "no move" in either log or model.

Definition 5.1 (Moves [15]). Let $L \in \mathcal{B}\left(A^{*}\right)$ be an event $\log$ over the activities $A$, and let $M$ be a model where $T$ is the set of tasks of the model, $A_{v}(M)$ is the set of observable activities of $M$, and $l$ is the labeling function between tasks and observable activities in $M$. For the sake of clarity, we abuse the notation writing $l(t)=\tau$ if $t \notin d o m(l)$, i.e., if $t$ is an invisible task.

- $\left(a_{L},\left(a_{M}, t\right)\right)$ is a move, where $a_{L} \in A \cup \gg,\left(a_{M}, t\right) \in\left(A_{v}(M) \cup \tau \times T\right) \cup \gg$, and $l(t)=a_{M}$.

- $(a,(a, t))$ is a synchronous move (also called move in both), where $a \in A, t \in T$, and $l(t)=a$. 


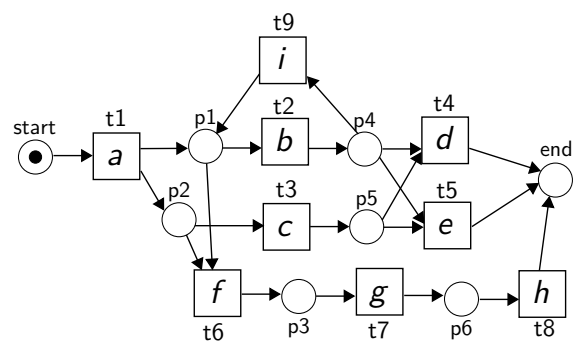

$$
\begin{aligned}
& \mathrm{a}=\text { Examination } \\
& \mathrm{b}=\text { Radiology } \\
& \mathrm{c}=\text { Update Record } \\
& \mathrm{d}=\text { Therapy } \\
& \mathrm{e}=\text { Home Care } \\
& \mathrm{f}=\text { Allergy Test } \\
& \mathrm{g}=\text { Chemotherapy } \\
& \mathrm{h}=\text { Post-Chemo } \\
& \mathrm{i}=\text { Operation }
\end{aligned}
$$

Figure 5.2: Model for a medical process, used as running example on this chapter.

- $(a, \gg)$ is a move on log, where $a \in A$.

- $(\gg,(a, t))$ is a move on model, where $(a, t) \in\left(A_{v}(M) \cup \tau \times T\right)$, and $l(t)=a$.

- A legal move is a move on log, a move on model, or a synchronous move. Otherwise, it is an illegal move. $A_{L M}$ denotes the set of possible legal moves between the model $M$ and $\log L$.

Given a sequence of moves $\gamma, \operatorname{row}_{L}^{\gg}(\gamma)$ denotes the sequence of $\log$ activities in $\gamma$, i.e., the first element. Similar, $\operatorname{row}_{M}^{\gg}(\gamma)$ and $\operatorname{row}_{T}^{\gg}(\gamma)$ denote the sequence of model activities and

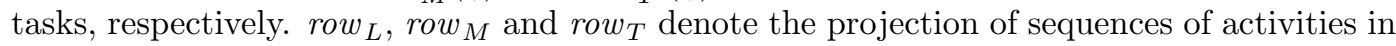
the log, model and tasks, filtering $\gg$.

Definition 5.2 (Alignment [15]). Let $\sigma_{L} \in L$ be a $\log$ trace and $\sigma_{M} \in \phi_{t}(M)$ a complete task sequence of model $M$. An alignment of $\sigma_{L}$ and $\sigma_{M}$ is a sequence of moves $\gamma \in A_{L M}{ }^{*}$ such that the sequence of $\log$ activities (ignoring $\gg$ ) yields $\sigma_{L}$, and the sequence of model tasks (ignoring $\gg$ ) yields $\sigma_{M}$, i.e., $\operatorname{row}_{L}(\gamma)=\sigma_{L}$ and $\operatorname{row}_{T}(\gamma)=\sigma_{M}$.

Let us consider a medical process for an oncological treatment in a hospital - this process will be used as running example during this chapter. Model $M$ in Figure 5.2 represents a possible model for this medical process. Assuming that the end state of the model is reached when place end in the model contains exactly one token, the model represents an infinite set of complete activity sequences, e.g., $\langle a, b, c, d\rangle,\langle a, c, b, d\rangle,\langle a, b, c, e\rangle,\langle a, c, b, e\rangle,\langle a, f, g, h\rangle$, $\langle a, b, i, c, b, e\rangle$. Given an unfitting trace $\sigma_{L}=\langle a, b, d, e\rangle$, Figure 5.3 shows some possible alignments between $\sigma_{L}$ and $M$.

The moves are represented vertically, e.g., the second move of $\gamma_{1}$ is $\left(\gg,\left(c, t_{3}\right)\right)$, indicating that the model performs $t_{3}$ while the log does not make any move. Note that after removing $\gg$, the projections of all moves in the model are by definition complete task sequences allowed by the model. This property is not always ensured by other conformance checking approaches. For example, given a trace and a process model, when using the approach in [80], the so-called missing tokens are added to allow activities that occur in the trace but are not 


\begin{tabular}{|c|c|c|c|c|c|c|c|c|c|c|c|c|c|c|c|c|c|c|c|}
\hline \multirow[b]{2}{*}{$\gamma_{1}=$} & a & & $\gg$ & $b$ & $d$ & \multirow{2}{*}{\multicolumn{2}{|c|}{$\gamma_{2}=$}} & $a$ & $b$ & $\gg$ & $d$ & $e$ & \multirow[b]{2}{*}{$\gamma_{3}=$} & $a$ & $\gg$ & $b$ & $d$ & $e$ & \\
\hline & $\begin{array}{l}a \\
t\end{array}$ & & $\begin{array}{c}c \\
t_{3}\end{array}$ & $\begin{array}{c}b \\
t_{2}\end{array}$ & $\gg$ & & & $\begin{array}{c}a \\
t_{1}\end{array}$ & $\begin{array}{c}b \\
t_{2}\end{array}$ & $\begin{array}{c}c \\
t_{3}\end{array}$ & $\gg$ & $\begin{array}{c}e \\
t_{5}\end{array}$ & & $\begin{array}{c}a \\
t_{1}\end{array}$ & $\begin{array}{c}c \\
t_{3}\end{array}$ & $\begin{array}{c}b \\
t_{2}\end{array}$ & $\begin{array}{c}d \\
t_{4}\end{array}$ & $\gg$ & \\
\hline & $a$ & $b$ & $\gg$ & $d$ & $e$ & & $a$ & $b$ & $d$ & $\gg$ & $e$ & & $a$ & $\gg$ & $\gg$ & $\gg$ & $b$ & $d$ & $e$ \\
\hline$=$ & $\begin{array}{c}a \\
t_{1}\end{array}$ & $\begin{array}{c}b \\
t_{2}\end{array}$ & $\begin{array}{c}c c \\
t_{3}\end{array}$ & $\begin{array}{c}d \\
t_{4}\end{array}$ & $\gg$ & $\gamma_{5}=$ & $\begin{array}{c}a \\
t_{1}\end{array}$ & $\begin{array}{c}b \\
t_{2}\end{array}$ & $\gg$ & $\begin{array}{c}c \\
t_{3}\end{array}$ & $\begin{array}{c}e \\
t_{5}\end{array}$ & $\gamma_{6}=$ & $\begin{array}{c}a \\
t_{1}\end{array}$ & $\begin{array}{c}f \\
t_{6}\end{array}$ & $\begin{array}{c}g \\
t_{7}\end{array}$ & $\begin{array}{c}h \\
t_{8}\end{array}$ & $\gg$ & $\gg$ & $\gg$ \\
\hline
\end{tabular}

Figure 5.3: Some alignments between trace $\sigma_{L}=\langle a, b, d, e\rangle$ and the model $M$ in Figure 5.2.

supposed to occur according to the model. The addition of such missing tokens introduces extra behavior that is not allowed in the original process model, thus overestimating its behavior.

In order to compare alignments and select the most appropriate one, costs are associated to undesirable moves and select an alignment with the lowest total costs. To quantify the costs of an alignment, a cost function $\delta$ is defined.

Definition 5.3 (Cost of alignment [15]). The cost function $\delta: A_{L M} \rightarrow \mathbb{N}$ assigns costs to legal moves. The cost of an alignment $\gamma \in A_{L M}{ }^{*}$ is the sum of all costs, i.e., $\delta(\gamma)=$ $\sum_{(x, y) \in \gamma} \delta(x, y)$.

Different scenarios may require different cost functions. The costs may depend on the nature of the activity, e.g., skipping a payment may be more severe than sending an email. Moreover, the severity assumed for a move on log and a move on model may be different, e.g., a system with constant recording problems should be more tolerant with activities skipped on the log. Abstracting from particular cases, we can define a standard cost function that assigns unit costs to moves in log or moves on model only.

Definition 5.4 (Standard Cost Function [15]). A standard cost function $\delta_{S}$ is defined such that:

- Synchronous move has cost 0 , i.e., $\delta_{S}(x,(x, t))=0$ for all $x \in A$.

- Move on $\log$ has cost 1 , i.e., $\delta_{S}(x, \gg)=1$.

- Move on model from a visible task has cost 1 , i.e., $\delta_{S}(\gg,(x, t))=1$.

- Move on model from an invisible task has cost 0 , i.e., $\delta_{S}(\gg,(\tau, t))=0$.

Using the standard cost function, the cost of alignment $\gamma_{1}$ is $\delta_{S}\left(\gamma_{1}\right)=\delta_{S}\left(a,\left(a, t_{1}\right)\right)+\delta_{S}(\gg$ ,$\left.\left(c, t_{3}\right)\right)+\delta_{S}\left(b,\left(b, t_{2}\right)\right)+\delta_{S}(d, \gg)+\delta_{S}\left(e,\left(e, t_{5}\right)\right)=0+1+0+1+0=2$. Note that the function returns the number of mismatches in the alignment. On the other hand, $\delta_{S}\left(\gamma_{6}\right)=6$. Hence, we conclude that $\gamma_{1}$ is close to the $\log$ trace $\sigma_{L}=\langle a, b, d, e\rangle$ than $\gamma_{6}$. 
Given a trace from an event log and a process model, we are interested in an activity sequence from the model that is most similar to the trace, i.e., the optimal alignment.

Definition 5.5 (Optimal Alignments [15]). We define the set of alignments $\Gamma_{\sigma_{L}, M}=\{\gamma \in$ $A_{L M}{ }^{*} \mid \gamma$ is an alignment between $\sigma_{L}$ and $\left.M\right\}$ to be all possible alignments between $\sigma_{L}$ and $M$. Accordingly, we define the set of optimal alignments as the set of all alignments with minimum cost, i.e., $\Gamma_{\sigma_{L}, M}^{o}=\left\{\gamma \in \Gamma_{\sigma_{L}, M} \mid \forall_{\gamma^{\prime} \in \Gamma_{\sigma_{L}, M}} \delta(\gamma) \leq \delta\left(\gamma^{\prime}\right)\right\}$.

It is easy to see that there can be more than one optimal alignment between a trace and a model. For example, $\left\{\gamma_{1}, \gamma_{2}, \gamma_{3}, \gamma_{4}, \gamma_{5}\right\}$ is the set of optimal alignments between the trace $\sigma_{L}=\langle a, b, d, e\rangle$ and the model $M$.

By definition, the task component of all alignments yields a complete task sequence of the model. Thus, given an optimal alignment $\gamma$ between $\sigma_{L}$ and $M, \operatorname{row}_{T}(\gamma)$ provides a complete tasks sequence that both perfectly fits $M$ and is closest to $\sigma_{L}$. In the running example, $\operatorname{row}_{T}\left(\gamma_{1}\right)=\left\langle t_{1}, t_{3}, t_{2}, t_{5}\right\rangle$ is one of the complete task sequences of $M$ that is most similar to trace $\langle a, b, d, e\rangle$.

Given a log and a model, constructing all optimal alignments between all traces in the log and the model is computationally expensive $[16,17]$. Thus, there are cases where computing all optimal alignments between traces and process models may not always be feasible in practice. Hence, instead of computing all optimal alignments between traces in the log and the model to obtain insights into deviations, one may also compute just some representative optimal alignments for each trace. In this chapter, we consider three approaches: one optimal alignment per trace, all optimal approaches, and a set of representative optimal alignments. We define three functions that provide optimal alignments between traces in the log and the model:

- $\Lambda_{M}^{*}: A_{L}^{*} \rightarrow \mathcal{P}\left(A_{L M}{ }^{*}\right)$ returns all optimal alignments between traces of $L$ and $M$, such that for all $\sigma_{L} \in L, \Lambda_{M}^{*}\left(\sigma_{L}\right)=\Gamma_{\sigma_{L}, M}^{o}$,

- $\Lambda_{M}^{1}: A_{L}^{*} \rightarrow A_{L M}{ }^{*}$ returns one optimal alignment between traces of $L$ and $M$, such that for all $\sigma_{L} \in L, \Lambda_{M}^{1}\left(\sigma_{L}\right) \in \Gamma_{\sigma_{L}, M}^{o}$, and

- $\Lambda_{M}^{R}: A_{L}^{*} \rightarrow \mathcal{P}\left(A_{L M}{ }^{*}\right)$ returns representatives of optimal alignments between traces of $L$ and $M$, such that for all $\sigma_{L} \in L, \Lambda_{M}^{R}\left(\sigma_{L}\right) \subseteq \Gamma_{\sigma_{L}, M}^{o}$.

In $[20,16,17]$ various approaches to obtain an optimal alignment between a trace and a model with respect to different cost functions are investigated. Given a trace $\sigma_{L}$ of $L$ and a model $M$, if there are multiple optimal alignments, $\Lambda_{M}^{1}$ chooses one of them according to other external criteria. With our previous example, suppose that $\Lambda_{M}^{1}$ selects an alignment that has the longest consecutive occurrence of synchronous moves in the beginning, $\Lambda_{M}^{1}\left(\sigma_{L}\right)=\gamma_{4}$.

In $[16,17]$, an $A^{\star}$-based algorithm is proposed to compute one optimal alignment between a trace and a model. The same algorithm can be extended to provide more than one optimal 


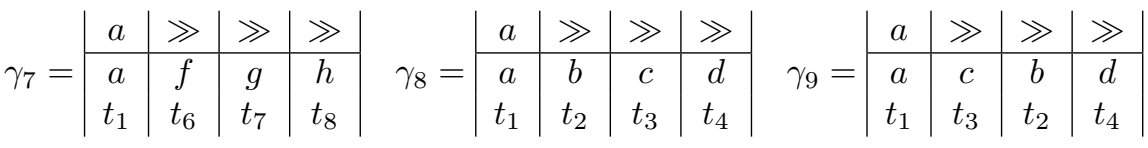

$$
\begin{aligned}
& \gamma_{10}=\left|\begin{array}{c|c|c|c|}
a & \gg & \gg & \gg \\
\hline a & c & b & e \\
t_{1} & t_{3} & t_{2} & t_{5}
\end{array}\right| \quad \gamma_{11}=\left|\begin{array}{c|c|c|c|}
a & \gg & \gg & \gg \\
\hline a & b & c & e \\
t_{1} & t_{2} & t_{3} & t_{5}
\end{array}\right|
\end{aligned}
$$

Figure 5.4: All optimal alignments between trace $\sigma_{L}=\langle a\rangle$ and the model $M$ in Figure 5.2.

alignment between them. Given a trace $\sigma_{L}$ of $L$ and a model $M$, the algorithm constructs one optimal alignment by computing a shortest path from the initial to the final state of the state space of the synchronous product between $\sigma_{L}$ and $M$. It is shown in [17] that all shortest paths from the initial to the final state of the state space yields an optimal alignment. For each state in the state space, the algorithm records a shortest path from the initial state to reach this state and thus, becomes the representative of all other shortest paths from the initial state to the state. An optimal alignment is constructed from a shortest path from the initial state to the final state that is also representing all other shortest paths that connect the same pair of states. By recording all represented shortest paths during state space exploration for each state, we can obtain all shortest paths from the initial to the final state of the state space (i.e., obtain all optimal alignments). Different representatives may represent different number of optimal alignments. Given a representative $\gamma \in \Lambda_{M}^{R}\left(\sigma_{L}\right), \operatorname{rep}_{M}(\gamma)$ denotes the number of optimal alignments represented by $\gamma$. Furthermore, due to possible pruning of state space, the total number of represented optimal alignments by the representatives is a lower bound of the total number of all optimal alignments, i.e., $\sum_{\gamma \in \Lambda_{M}^{R}\left(\sigma_{L}\right)} \operatorname{rep}_{M}(\gamma) \leq$ $\left|\Gamma_{\sigma_{L}, M}^{o}\right|$. The interested reader is referred to $[16,17,15]$ for details on the constructed state space with the $A^{\star}$-based algorithm approach.

Take for example a trace $\sigma_{L}=\langle a\rangle$. All optimal alignments between the trace and the medical model $M$ are shown in Figure 5.4. Given a possible function $\Lambda^{R}, \Lambda^{R}\left(\sigma_{L}\right)=$ $\left\{\gamma_{7}, \gamma_{9}, \gamma_{10}\right\}$ where $\operatorname{rep}_{M}\left(\gamma_{7}\right)=1\left(\gamma_{7}\right.$ represents $\left.\left\{\gamma_{7}\right\}\right), \operatorname{rep}\left(\gamma_{9}\right)=2\left(\gamma_{9}\right.$ represents $\left.\left\{\gamma_{8}, \gamma_{9}\right\}\right)$, and $\operatorname{rep}\left(\gamma_{10}\right)=2\left(\gamma_{10}\right.$ represents $\left.\left\{\gamma_{10}, \gamma_{11}\right\}\right)$.

For simplicity, in the remainder we omit the model notation $M$ in functions $\Lambda_{M}^{*}, \Lambda_{M}^{1}$, $\Lambda_{M}^{R}$, and $\operatorname{rep}_{M}$ whenever the context is clear. Note that in cases where a process model has duplicate tasks (more than one task to represent an activity) or invisible tasks (tasks whose execution are not logged), approaches to construct alignments (e.g., [20, 16]) keep the mapping from all model moves to the tasks they correspond to. Hence, given an alignment of a trace and such models, we know exactly which task is executed for each model move. We refer to $[20,16]$ for further details on how such mapping is constructed. 


\subsection{Precision based on Alignments}

The technique described in the previous section provides optimal alignments for each trace in the log. This section presents a technique to compute precision based on the use of these optimal alignments. Like the approach on Chapter 3, the behavior observed in the log is used to traverse the modeled behavior, detecting escaping arcs for possible points of imprecision. However, whereas in Chapter 3 is based on model replay directly from the log, the approach presented here uses the alignments as a more faithful representation of the observed behavior. The advantages are manifold. First of all, traces in the log do not need to be completely fitting. In Chapter 3, the non-fitting parts are simply ignored. For most real-life situations, this implies that only a fraction of the event log can be used for computing precision. Second, the existence of indeterminism in the model poses no problems when using the alignments. Finally, the use of alignments instead of log-based model replay improves the robustness of conformance checking. The remainder of this section is devoted to explain how precision can be calculated from the alignments. In particular, we consider the precision checked from one alignment, all alignments, and representative alignments. To illustrate the three approaches, in the remainder of the section we use the following running example: the model $M$ shown in Figure 5.2 and the $\log L=\left[\sigma_{1}, \sigma_{2}, \sigma_{3}, \sigma_{4}, \sigma_{5}\right]$, containing the 5 traces that appear in in Table 5.1. The table also provides the optimal alignments for the traces in $L$.

\subsubsection{Precision from 1-Alignment}

Like Chapter 3, precision is estimated by confronting model and log behavior: escaping arcs between the model and the log (i.e., situations where the model allows more behavior than the one reflected in the log) are detected by juxtaposing behavior observed in the log and the one allowed by the model. This juxtaposition is done in terms of an automaton: first, an automaton is built from the alignments. Then, the automaton is enhanced with behavioral information of the model. Finally, the enhanced automaton is used to compute the precision. In order to build the automaton, observed behavior must be determined in terms of model perspective, i.e., we consider the optimal alignments of each trace in the $\log$ for this purpose. For example, given the running example $L$ and $M$, the trace $\sigma_{1}$ has 5 optimal alignments, $\left.\gamma_{1 a}, \gamma_{1 b}, \gamma_{1 c}, \gamma_{1 d}, \gamma_{1 e}\right\}$, shown in Table 5.1. However, in 1-alignment only one alignment is considered. For this example, we assume that the alignment assigned to $\sigma_{1}$ by $\Lambda^{1}$ based on an external criterion corresponds to $\gamma_{1 a}$, i.e., $\Lambda^{1}\left(\sigma_{1}\right)=\gamma_{1 a}$. On the other hand, traces $\sigma_{2} \ldots \sigma_{5}$ are perfectly fitting and have only one optimal alignment containing only synchronous moves. Given an alignment $\gamma$, in order to build the automaton, we only consider the projection of tasks moves, i.e., row $w_{T}(\gamma)$. In this example, the sequences used as observed behavior are $\left\langle t_{1}, t_{6}, t_{7}, t_{8}\right\rangle,\left\langle t_{1}, t_{2}, t_{3}, t_{4}\right\rangle,\left\langle t_{1}, t_{3}, t_{2}, t_{5}\right\rangle,\left\langle t_{1}, t_{6}, t_{7}, t_{8}\right\rangle$ and $\left\langle t_{1}, t_{2}, t_{9}, t_{2}, t_{3}, t_{4}\right\rangle$. We use $\operatorname{row}_{T}\left(\Lambda^{1}\right)_{L}$ to denote the application of function row $_{T}$ on all the alignments provided by the functions $\Lambda^{1}$ for the traces in $\log L$. We can omit the subindex $L$ whenever the context is clear. Note that, by definition, any alignment projection $\operatorname{row}_{T}(\gamma)$ is a valid complete firing sequence of the model. 


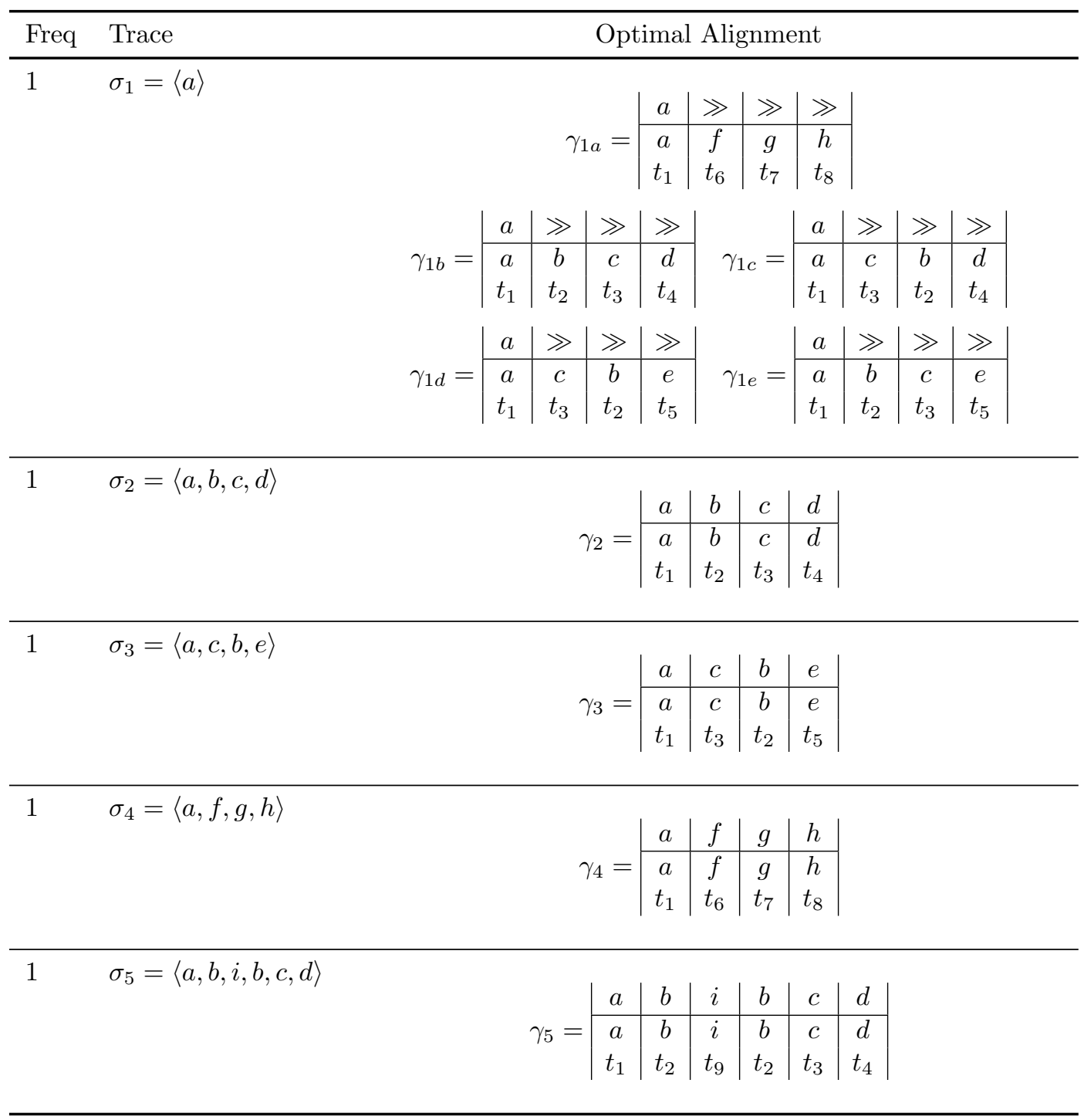

Table 5.1: Optimal alignments of $\log \left[\sigma_{1}, \sigma_{2}, \sigma_{3}, \sigma_{4}, \sigma_{5}\right]$ and the medical model $M$ of Figure 5.2 
Similar to Chapter 3, the automaton is built considering all the prefixes for the sequences in $\operatorname{row}_{T}\left(\Lambda^{1}\right)$ as the states. For instance, given a sequence $\left\langle t_{1}, t_{2}, t_{3}, t_{4}\right\rangle$ resulting of $\operatorname{row}_{T}\left(\Lambda^{1}\right)\left(\sigma_{2}\right)$, the states considered are \langle\rangle$,\left\langle t_{1}\right\rangle,\left\langle t_{1}, t_{2}\right\rangle,\left\langle t_{1}, t_{2}, t_{3}\right\rangle$ and $\left\langle t_{1}, t_{2}, t_{3}, t_{4}\right\rangle$. We denote as $\bullet\left(\operatorname{row}_{T}(\gamma)\right)$ the set of prefixes of the tasks sequence of the alignment $\gamma$ and as - $\left(\right.$ row $\left._{T}\left(\Lambda^{1}\right)\right)$ the multiset of prefixes of the the tasks sequences of all alignments in $\Lambda^{1}$.

Definition 5.6 (Prefix Automaton of the 1-Alignment). Let $L \in \mathcal{B}\left(A^{*}\right)$ be an event $\log$, let $M$ be a model with tasks $T$, and let $\operatorname{row}_{T}\left(\Lambda^{1}\right)$ be the alignments between them projected on the model tasks. We define the prefix automaton of the 1 -Alignment $\mathcal{A}_{\Lambda^{1} M}=\left(S, T, \nearrow, \omega, s_{0}\right)$ such that:

- the set of states correspond to the set of prefixes of the alignments projected on the model tasks, i.e., $S=\left\{\sigma \mid \sigma \in \bullet\left(\operatorname{row}_{T}\left(\Lambda^{1}\right)\right)\right\}$.

- the set of labels correspond to the set of tasks $T$.

- the arcs $\nearrow \subseteq(S \times T \times S)$ define the concatenation between states and tasks, i.e., $\nearrow=\{(\sigma, t, \sigma \cdot\langle t\rangle) \mid \sigma \in S \wedge \sigma \cdot\langle t\rangle \in S\}$.

- the function that determines the weight of a state is determined by the number of occurrences of the state in the multiset of prefixes of the tasks sequences, i.e., $\omega(\sigma)=$ • $\left(\operatorname{row}_{T}\left(\Lambda^{1}\right)\right)(\sigma)$ for all $\sigma \in S$.

- the initial state $s_{0}$ corresponds with the empty prefix \langle\rangle .

Figure 5.5 shows the resulting automata for the running exemple $L$ using the function $\Lambda^{1}$ (only the white states). For example, the weight of the state $\left\langle t_{1}\right\rangle$ is greater than the weight of $\left\langle t_{1}, t_{3}\right\rangle$ because there are more tasks sequences with the prefix $\left\langle t_{1}\right\rangle$ (all 5 sequences), than the ones with prefix $\left\langle t_{1}, t_{3}\right\rangle$ (only the sequence $\left\langle t_{1}, t_{3}, t_{2}, t_{5}\right\rangle$ contains that prefix).

Once the observed behavior has been determined in terms of an automaton, the confrontation with the actual modeled behavior is required in order to determine the precision. For each state of the automaton, we compute its set of modeled tasks, i.e., possible direct successor tasks according to the model $(\bmod )$, and then compare it with the set of observed tasks, i.e., tasks really executed in the log (obs)(cf. Definition 3.4). Let us consider, for example, state $\left\langle t_{1}, t_{2}, t_{3}\right\rangle$ of automaton in Figure 5.5. The set of observed tasks of the state is $\operatorname{obs}\left(\left\langle t_{1}, t_{2}, t_{3}\right\rangle\right)=\left\{t_{4}\right\}$, i.e., for all traces with prefix $\left\langle t_{1}, t_{2}, t_{3}\right\rangle$, their direct successor is only $t_{4}$. The set of modeled tasks for the state is $\bmod \left(\left\langle t_{1}, t_{2}, t_{3}\right\rangle\right)=\left\{t_{4}, t_{5}, t_{9}\right\}$ because after performing the sequence of tasks $\left\langle t_{1}, t_{2}, t_{3}\right\rangle$, the model allows to do $t_{4}, t_{5}$ or $t_{9}$. Note that, by definition of alignment, $\operatorname{obs}(s) \subseteq \bmod (s)$, i.e., the set of executed tasks of a given state is always a subset of all available tasks according to the model.

The arcs that are modeled according to the model, but do not occur in the event log according to the alignments, are used to collect the escaping arcs of the system, i.e., an arcs that escapes from the observed behavior. The tasks on the escaping arcs and the states reached are called escaping tasks and escaping states respectively. In Figure 5.5 


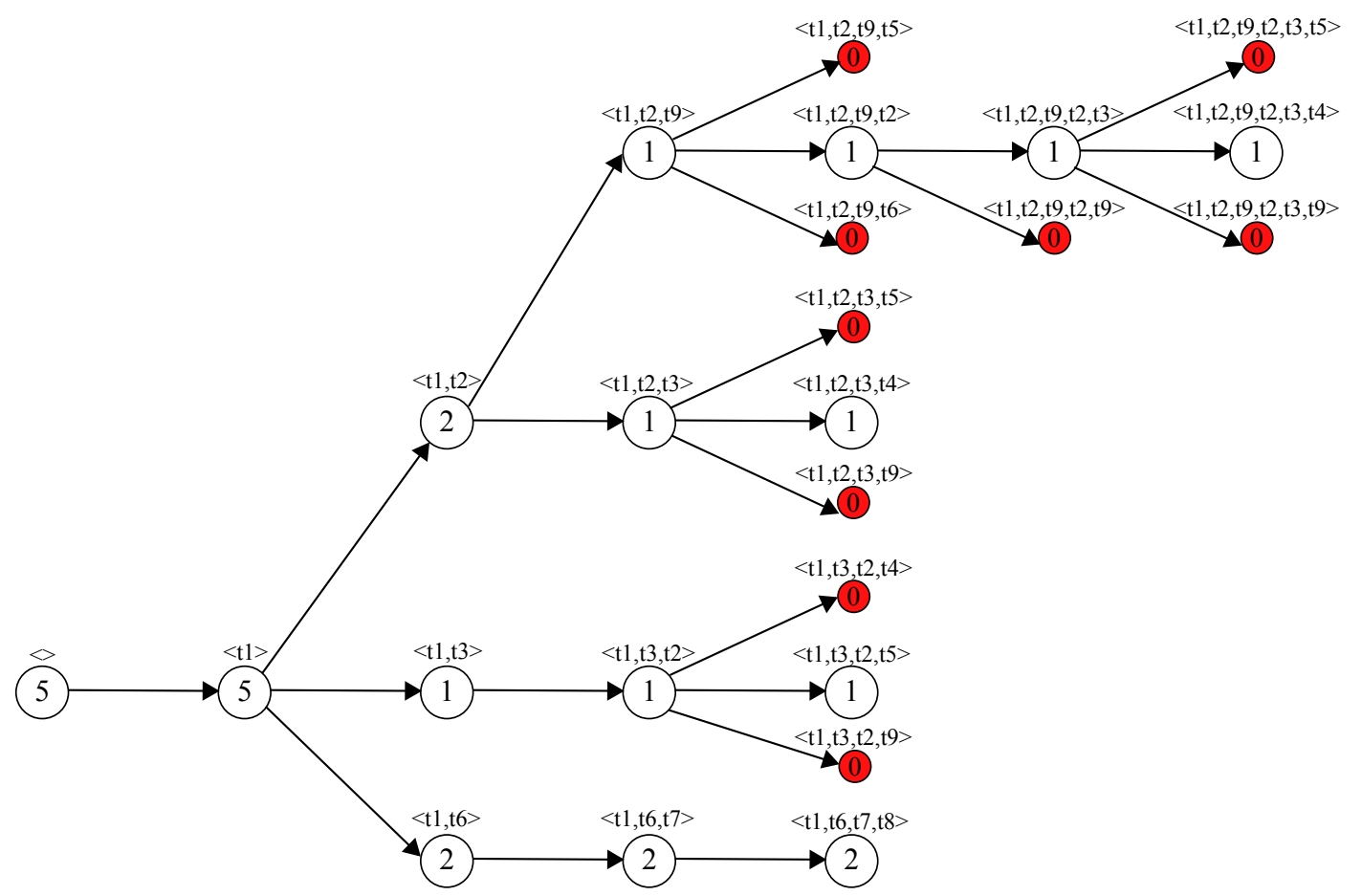

Figure 5.5: Automaton from 1-alignments between model $M$ and $\log L$.

the escaping states are in color. For example, the escaping tasks of the state $\left\langle t_{1}, t_{2}, t_{3}\right\rangle$ are $\left\{t_{4}, t_{5}, t_{9}\right\} \backslash\left\{t_{4}\right\}=\left\{t_{5}, t_{9}\right\}$. The computation and analysis of these escaping arcs are the cornerstone of the precision checking technique presented in this thesis. All identified escaping arcs can be analyzed and further used to correct the model and make it more precise. Furthermore, in order to globally estimate precision, these escaping arcs in turn are weighted and normalized defining a metric to measure precision called 1-align precision metric.

Definition 5.7 (1-Align Precision metric). Let $\mathcal{A}_{\Lambda^{1} M}=\left(S, T, \nearrow, \omega, s_{0}\right)$ be the prefix automaton of the alignments in $\Lambda^{1}$ enhanced with the behavior of the model $M$. The metric 1-Align Precision estimates the precision of the system comparing, for each state in $S$, the number of escaping arcs with the number of allowed arcs. The numbers are weighted according to the importance of the state. Formally:

$$
a_{p}^{1}\left(\mathcal{A}_{\Lambda^{1} M}\right)=1-\frac{\sum_{s \in S} \omega(s) \cdot|\operatorname{esc}(s)|}{\sum_{s \in S} \omega(s) \cdot|\bmod (s)|}
$$

For example, the precision for the automaton derived from $\Lambda^{1}$ shown in Figure 5.5 is 
0.79 .

\subsubsection{Precision from All-Alignment}

As experiments show, the use of one alignment is an effective and efficient alternative for precision checking. However, the approach relies on selecting one of optimal alignments, and therefore, it may detect more escaping arcs. Let us consider the model in Figure 5.6, and a $\log$ with only one trace $\langle a, d\rangle$.

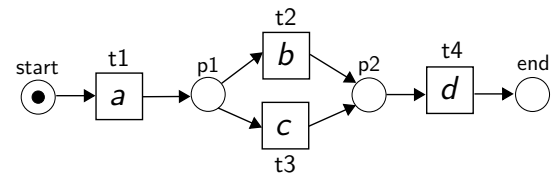

Figure 5.6: Model with two optimal alignments for the trace $\langle a, d\rangle$, one with move on the model $\left(b, t_{2}\right)$, and the other with one move on model $\left(c, t_{3}\right)$.

There are two possible optimal alignments: one includes a move on the model $\left(b, t_{2}\right)$, and the other one move on model $\left(c, t_{3}\right)$, while $\left(a, t_{1}\right)$ and $\left(d, t_{4}\right)$ are synchronous moves. If we select the first alignment for computing the precision, $\left\langle t_{1}, t_{3}\right\rangle$ is then considered an escaping tasks. On the other hand, if we select the second alignment, $\left\langle t_{1}, t_{2}\right\rangle$ becomes an escaping tasks. In other words, decisions in the fitness dimension are affecting the precision dimension. As $[80,79]$ states, the analysis of one conformance dimension should be independent from the others. Therefore, given that both alignments have the same cost, both alignments must be considered for precision dimension, while the mismatches produced by the moves on model must be evaluated in the fitness phase. Following with the running example of Table 5.1, we consider the following 9 complete transition sequences to represent the observed behavior: $\operatorname{row}_{T}\left(\gamma_{1 a}\right)=\left\langle t_{1}, t_{6}, t_{7}, t_{8}\right\rangle, \operatorname{row}_{T}\left(\gamma_{1 b}\right)=\left\langle t_{1}, t_{2}, t_{3}, t_{4}\right\rangle, \operatorname{row}_{T}\left(\gamma_{1 c}\right)=\left\langle t_{1}, t_{3}, t_{2}, t_{4}\right\rangle$, $\operatorname{row}_{T}\left(\gamma_{1 d}\right)=\left\langle t_{1}, t_{3}, t_{2}, t_{5}\right\rangle, \operatorname{row}_{T}\left(\gamma_{1 e}\right)=\left\langle t_{1}, t_{2}, t_{3}, t_{5}\right\rangle, \operatorname{row}_{T}\left(\gamma_{2}\right)=\left\langle t_{1}, t_{2}, t_{3}, t_{4}\right\rangle, \operatorname{row}_{T}\left(\gamma_{3}\right)=$ $\left\langle t_{1}, t_{3}, t_{2}, t_{5}\right\rangle, \operatorname{row}_{T}\left(\gamma_{4}\right)=\left\langle t_{1}, t_{6}, t_{7}, t_{8}\right\rangle$, and $\operatorname{row}_{T}\left(\gamma_{5}\right)=\left\langle t_{1}, t_{2}, t_{9}, t_{2}, t_{3}, t_{4}\right\rangle$,

Notice that, constructing an automaton from all the alignments using the approach on the previous section introduces a bias on the weighting functions: $\log$ traces associated with more optimal alignments would have more weight. For example, $\log$ trace $\sigma_{1}$ would have five times more influence in the measure $-\operatorname{row}_{T}\left(\gamma_{1 a}\right), \operatorname{row}_{T}\left(\gamma_{1 b}\right), \operatorname{row}_{T}\left(\gamma_{1 c}\right), \operatorname{row}_{T}\left(\gamma_{1 d}\right)$ and $\operatorname{row}_{T}\left(\gamma_{1 e}\right)$ - than $\sigma_{2}-\operatorname{row}_{T}\left(\gamma_{2}\right)$. In other words, in order to have an unbiased precision measure, the weight of each optimal alignment associated with a log trace must be normalized, such that each trace of the log has the same importance within the observed behavior. For that, we define the weight of an alignment, and we redefine the weight of the states in the prefix automaton.

Definition 5.8 (Weight of Alignment). Let $M$ be a model, let $L$ be an event log, let $\sigma_{L}$ be a trace in $L$, let $L\left(\sigma_{L}\right)$ be the frequency of $\sigma_{L}$, and let $\gamma \in \Lambda_{M}^{*}\left(\sigma_{L}\right)$ be one of the optimal 
alignments between $\sigma_{L}$ and $M$. The weight of $\gamma$ is defined as $\omega(\gamma)=L\left(\sigma_{L}\right) \cdot 1 /\left|\Lambda_{M}^{*}\left(\sigma_{L}\right)\right|$, i.e., the weight is split equally among all the alignments of the log trace, taking into account the frequency of the trace withing the log. Given the prefix automaton $\mathcal{A}_{\Lambda^{*} M}=\left(S, T, \nearrow, \omega, s_{0}\right)$, the weight of a state $s \in S$ is defined as:

$$
\omega(s)=\sum_{\forall \gamma \in \Lambda^{*}} \omega(\gamma) \text { if } s \text { is a prefix of } \operatorname{row}_{T}(\gamma) \text { (or } 0 \text { otherwise) }
$$

For example, the weight of the alignment $\gamma_{1 a}$ of trace $\sigma_{1}$ is $1 \cdot 1 / 5=0.2$, while the weight of $\gamma_{2}$ is $1 \cdot 1 / 1=1$. Figure 5.7 shows the resulting automata for the running exemple $L$ and $M$ using the function $\Lambda^{*}$. For example, the weight of the state $\left\langle t_{1}, t_{6}\right\rangle$ is 1 from $\gamma_{4}$ plus 0.2 from $\gamma_{1 a}$, i.e., 1.2 .

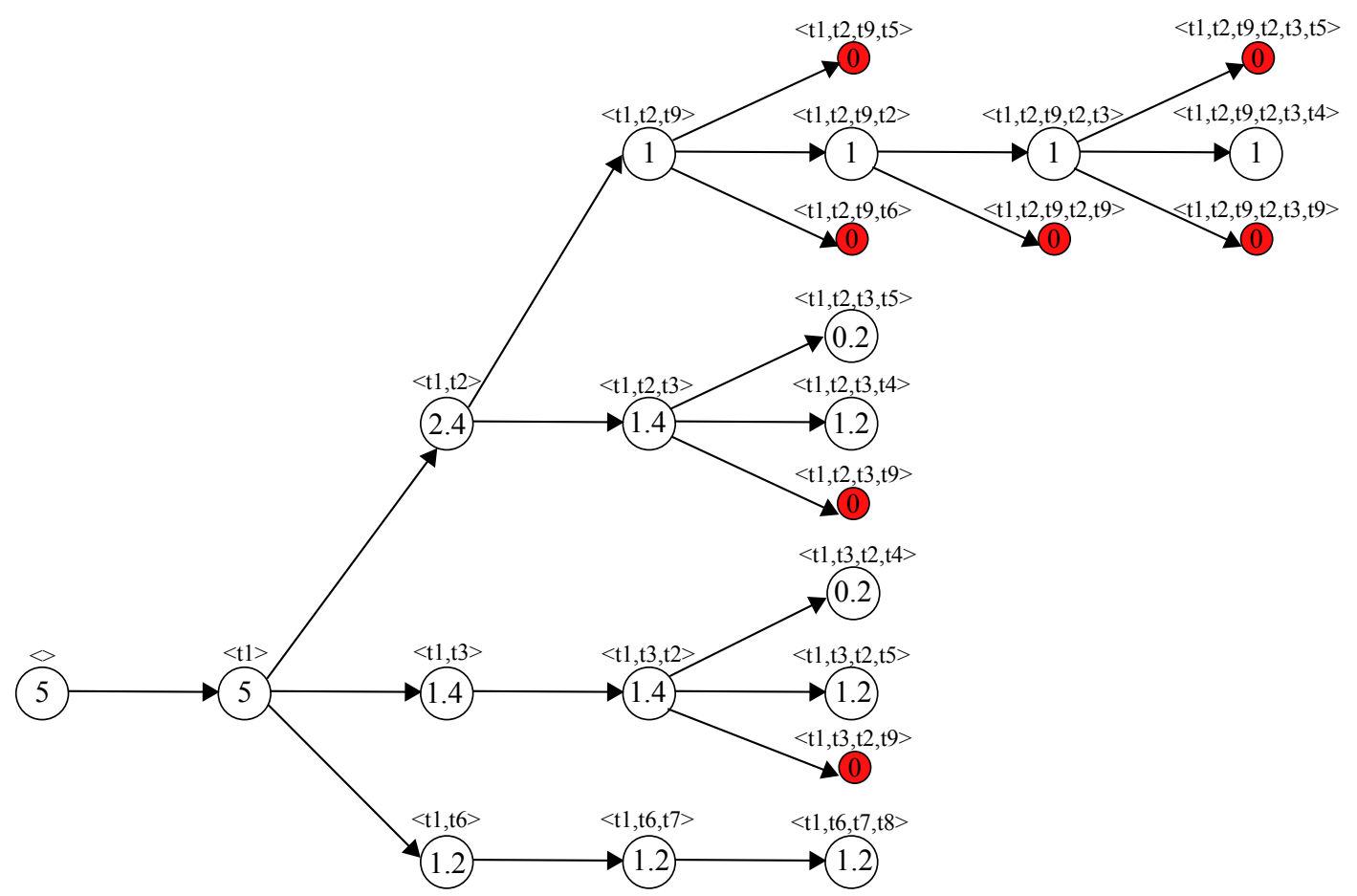

Figure 5.7: Automaton from all-alignments between model $M$ and $\log L$.

As it is done with 1-align precision metric, the all-align metric is formalized as:

Definition 5.9 (All-Align Precision metric). Let $\mathcal{A}_{\Lambda^{*} M}=\left(S, T, \nearrow, \omega, s_{0}\right)$ be the prefix automaton of the alignments in $\Lambda^{*}$ enhanced with the behavior of the model $M$. The metric All-Align Precision estimates the precision of the system comparing, for each state in $S$, 
the number of escaping arcs with the number of allowed arcs. The numbers are weighted according to the importance of the state. Formally:

$$
a_{p}^{*}\left(\mathcal{A}_{\Lambda^{*} M}\right)=1-\frac{\sum_{s \in S} \omega(s) \cdot|\operatorname{esc}(s)|}{\sum_{s \in S} \omega(s) \cdot|\bmod (s)|}
$$

\subsubsection{Precision from Representative-Alignment}

Given a $\log$ trace and a process model, $\Lambda^{*}$ provides all optimal alignments. However, as shown in [18], it is an expensive option in terms of computation time. The use of only one alignment per trace (i.e., $\Lambda^{1}$ ) solves this issue in cases where time is a priority, but it may sacrifice accuracy. As a trade-off between time and accuracy, in this section we propose precision measurement based on representatives of all optimal alignments $\Lambda^{R}$ (cf. Section 5.2). In this section, we revisit the precision measurement to include this notion.

\begin{tabular}{llcl}
\hline Freq & Trace & $\Lambda^{R}$ & rep \\
\hline 1 & $\sigma_{1}=\langle a\rangle$ & $\gamma_{1 a}$ & 1 \\
& & $\gamma_{1 c}$ & 2 \\
& & $\gamma_{1 d}$ & 2 \\
\hline 1 & $\sigma_{2}=\langle a, b, c, d\rangle$ & $\gamma_{2}$ & 1 \\
\hline 1 & $\sigma_{3}=\langle a, c, b, e\rangle$ & $\gamma_{3}$ & 1 \\
\hline 1 & $\sigma_{4}=\langle a, f, g, h\rangle$ & $\gamma_{4}$ & 1 \\
\hline 1 & $\sigma_{5}=\langle a, b, i, b, c, d\rangle$ & $\gamma_{5}$ & 1 \\
\hline
\end{tabular}

Table 5.2: Optimal representative alignments of $\log L=\left[\sigma_{1}, \sigma_{2}, \sigma_{3}, \sigma_{4}, \sigma_{5}\right]$ and the medical model $M$ of Figure 5.2

Table 5.2 show the optimal representative alignments assigned to each trace of the running example, and the number of optimal alignments they represent.

The construction of the prefix automaton and the definition of escaping arcs for the representative alignments are the same as the or for all the alignments shown in the previous section, except for the weighting function. The weight of an alignment $\gamma$ needs to be proportional to the number of alignments represented by $\gamma$, i.e., $\operatorname{rep}(\gamma)$.

Definition 5.10 (Weight of Alignment). Let $M$ be a model, let $L$ be an event log, let $\sigma_{L}$ be a trace in $L$, let $L\left(\sigma_{L}\right)$ be the frequency of $\sigma_{L}$, and let $\gamma \in \Lambda_{M}^{R}\left(\sigma_{L}\right)$ be one of the optimal representative alignments between $\sigma_{L}$ and $M$, where $\operatorname{rep}(\gamma)$ are the alignments represented by $\gamma$. The weight of $\gamma$ is defined as:

$$
\omega(\gamma)=L\left(\sigma_{L}\right) \cdot \operatorname{rep}(\gamma) / \sum_{\gamma^{\prime} \in \Lambda^{R}\left(\sigma_{L}\right)} \operatorname{rep}\left(\gamma^{\prime}\right)
$$


For instance, let us consider the optimal representative alignment $\gamma_{1 c}$ for the $\log$ trace $\sigma_{1}$. The number of alignments represented by $\gamma_{1 c}$ is $\operatorname{rep}\left(\gamma_{1 c}\right)=2$. The total number of optimal alignments represented by the representative alignments associated with $\sigma_{1}$ is $\sum_{\gamma^{\prime} \in \Lambda^{R}\left(\sigma_{1}\right)} \operatorname{rep}\left(\gamma^{\prime}\right)=5$. Hence, the weight $\omega\left(\gamma_{1 c}\right)=1 \cdot 2 / 5=0.4$. On the other hand, let us consider $\gamma_{5}$, the only optimal alignment associated with $\sigma_{5}$. The representative alignment $\gamma_{5}$ represents 1 optimal alignment. Since the number of all optimal alignments represented is $\sum_{\gamma^{\prime} \in \Lambda^{R}\left(\sigma_{5}\right)} \operatorname{rep}\left(\gamma^{\prime}\right)=1$, the weight of $\gamma_{5}$ is $\omega\left(\gamma_{5}\right)=1 \cdot 1 / 1=1$.

Figure 5.8 shows the resulting automata for the running exemple $L$ and $M$ using the function $\Lambda^{R}$. For example, the weight of the state $\left\langle t_{1}, t_{3}\right\rangle$ is 1 from $\gamma_{3}$, plus 0.4 from $\gamma_{1 c}$ (represents 2 alignments of 5 ), plus 0.4 from $\gamma_{1 d}$ (represents 2 alignments of 5), i.e., 1.8.

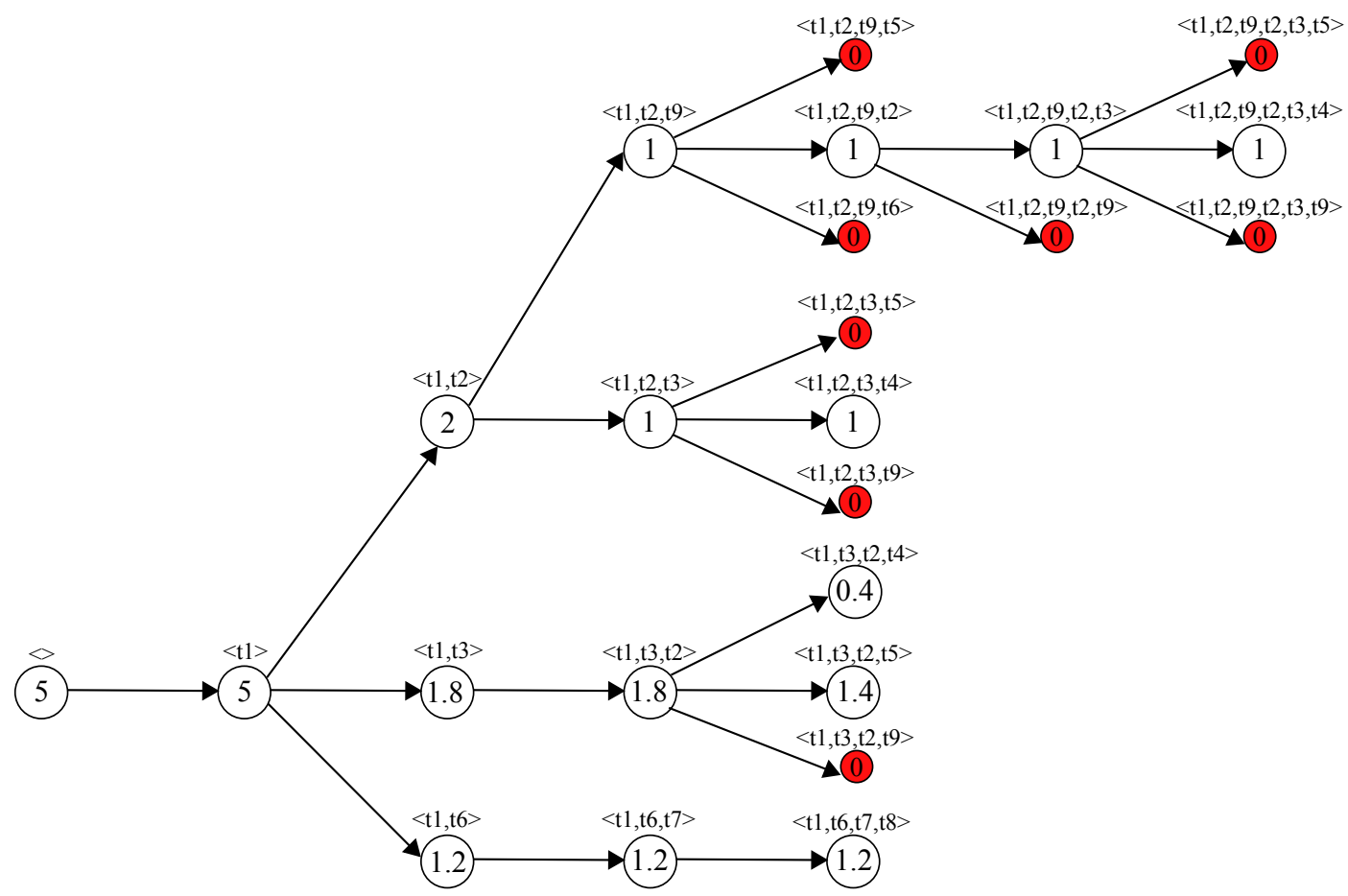

Figure 5.8: Automaton from representative-alignments between model $M$ and $\log L$.

As it is done with 1-align and all-align precision metric, the rep-align metric is formalized as:

Definition 5.11 (Rep-Align Precision metric). Let $\mathcal{A}_{\Lambda^{R} M}=\left(S, T, \nearrow, \omega, s_{0}\right)$ be the prefix automaton of the alignments in $\Lambda^{R}$ enhanced with the behavior of the model $M$. The metric All-Align Precision estimates the precision of the system comparing, for each state in $S$, 
the number of escaping arcs with the number of allowed arcs. The numbers are weighted according to the importance of the state. Formally:

$$
a_{p}^{R}\left(\mathcal{A}_{\Lambda^{R} M}\right)=1-\frac{\sum_{s \in S} \omega(s) \cdot|\operatorname{esc}(s)|}{\sum_{s \in S} \omega(s) \cdot|\bmod (s)|}
$$

Note that there can be more than one ways to compute representative alignments from a given model and a trace. Given an event log and a model, the selection of representative alignments between each trace in the log and the model obviously influences the automata that can be constructed between the log and the model.

\subsection{Abstractions for the Precision based on Alignments}

The approach presented in Section 5.3 uses the prefixes of complete tasks sequences to represent states of the automaton. This implies that given a complete tasks sequence $\sigma$, other sequences with slightly different permutation of tasks are placed in different branches of constructed automaton than $\sigma$. Given a process model that allows many possible interleaving of tasks, the approach can only provide a perfect precision value if all permutations of the interleaving activities are been observed. This requirement may be too restrictive in some cases.

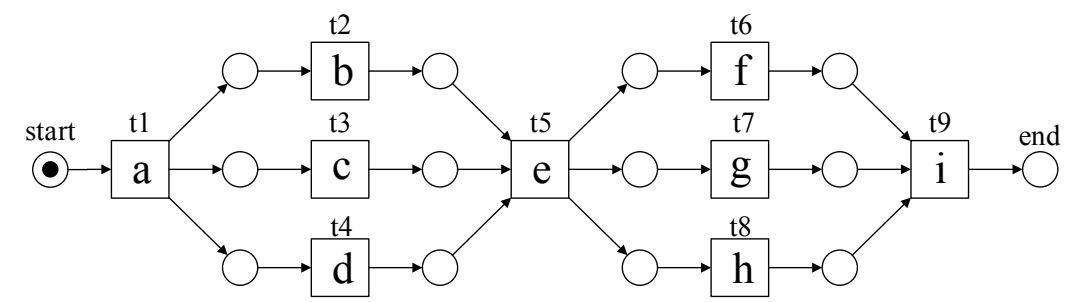

Figure 5.9: Process model that allows the interleaving of two blocks of tasks: $t_{2}, t_{3}, t_{4}$ and $t_{6}, t_{7}, t_{8}$.

For example, let us consider the process model in Figure 5.9 and the log in Table 5.3. The model allows for the interleaved execution of $t_{2}, t_{3}$ and $t_{4}$. This behavior is also observed in the $\log$, containing all possible permutations of $t_{2}, t_{3}$ and $t_{4}$. The model also allows the interleaving of $t_{6}, t_{7}$ and $t_{8}$, and all possible permutations of $t_{6}, t_{7}$ and $t_{8}$ are also observed in the log. One may expect a perfect precision of 1 for such model and log. However, given the presented approach, the precision is 0.8 . The automaton of Figure 5.10 shows the escaping arcs detected. Notice that prefix $\left\langle t_{1}, t_{2}, t_{3}\right\rangle$ of $\left\langle t_{1}, t_{2}, t_{3}, t_{4}, t_{5}, t_{6}, t_{7}, t_{8}, t_{9}\right\rangle$ and prefix $\left\langle t_{1}, t_{3}, t_{2}\right\rangle$ of $\left\langle t_{1}, t_{3}, t_{2}, t_{4}, t_{5}, t_{7}, t_{6}, t_{8}, t_{9}\right\rangle$ represent two different states even when the 


\begin{tabular}{lll}
\hline Freq & Trace & $\operatorname{row}_{T}(\gamma)$ \\
\hline 1 & $\langle a, b, c, d, e, f, g, h, i\rangle$ & $\left\langle t_{1}, t_{2}, t_{3}, t_{4}, t_{5}, t_{6}, t_{7}, t_{8}, t_{9}\right\rangle$ \\
\hline 1 & $\langle a, b, d, c, e, f, h, g, i\rangle$ & $\left\langle t_{1}, t_{2}, t_{4}, t_{3}, t_{5}, t_{6}, t_{8}, t_{7}, t_{9}\right\rangle$ \\
\hline 1 & $\langle a, c, b, d, e, g, f, h, i\rangle$ & $\left\langle t_{1}, t_{3}, t_{2}, t_{4}, t_{5}, t_{7}, t_{6}, t_{8}, t_{9}\right\rangle$ \\
\hline 1 & $\langle a, c, d, b, e, g, h, f, i\rangle$ & $\left\langle t_{1}, t_{3}, t_{4}, t_{2}, t_{5}, t_{7}, t_{8}, t_{6}, t_{9}\right\rangle$ \\
\hline 1 & $\langle a, d, b, c, e, h, f, g, i\rangle$ & $\left\langle t_{1}, t_{4}, t_{2}, t_{3}, t_{5}, t_{8}, t_{6}, t_{7}, t_{9}\right\rangle$ \\
\hline 1 & $\langle a, d, c, b, e, h, g, f, i\rangle$ & $\left\langle t_{1}, t_{4}, t_{3}, t_{2}, t_{5}, t_{8}, t_{7}, t_{6}, t_{9}\right\rangle$ \\
\hline
\end{tabular}

Table 5.3: Event log for the model in Figure 5.9

executed tasks and their frequency in both prefixes are the same. For the given example, the minimum number of traces necessary to reach a precision of 1 is 36 . This number increases exponentially with the increasing degree of concurrency of the considered model. In such cases, some level of abstraction in the way states are represented is desirable. In Section 5.4.1 we propose an approach to abstract from the order of the tasks to compute the precision, dealing with the possible incompleteness of the log.

Moreover, notice that the automaton is constructed considering the prefixes of the complete tasks sequences. However, this may introduce a bias on the direction, i.e., the tasks executed in the past are the ones that determine the current state. A reasonable alternative is to consider the future tasks to determine the current state. In Section 5.4.2 we propose to use the future to construct the automaton, in order to deal with the possible bias produced by the direction used to compute precision.

\subsubsection{Abstraction on the Order}

In [12], the authors describe an approach to extract a transition system from the log. The proposed approach consider a set of possible abstractions and filters on the trace in order to determine the states of the transition system. In particular, they propose the use of sets, multisets, and sequences of activities as state representations. In a similar way, in this section we propose two possible state representations for precision checking that can be chosen depending on the desired level of abstractions:

- Ordered: A state is a sequence of tasks. This is the same representation as the one used in Section 5.3. For example, the states for prefix $\left\langle t_{1}, t_{2}, t_{3}\right\rangle$ and $\left\langle t_{1}, t_{3}, t_{2}\right\rangle$ are different.

- Unordered: A state is a multiset of tasks, i.e., the order among tasks does not matter, but the number of executions of each task does. For example, the states for $\left\langle t_{1}, t_{2}, t_{3}\right\rangle$ and $\left\langle t_{1}, t_{3}, t_{2}\right\rangle$ are the same, i.e., $\left[t_{1}, t_{2}, t_{3}\right]$. However, the states for $\left\langle t_{1}, t_{2}, t_{9}\right\rangle$ and 
$\left\langle t_{1}, t_{2}, t_{9}, t_{2}\right\rangle$ are not the same, i.e., $\left[t_{1}, t_{2}, t_{9}\right]$ and $\left[t_{1}, t_{2}{ }^{2}, t_{9}\right]$ respectively, because the number of occurrences of each task matters.

Figure 5.11 show the 1-alignment automaton for the medical process, considering the multiset state representation. This automaton contains differences with respect to its ordered homologous (cf. Figure 5.5). For example, instead of having two states $\left\langle t_{1}, t_{2}, t_{3}\right\rangle$ and $\left\langle t_{1}, t_{3}, t_{2}\right\rangle$ for prefixes $\left\langle t_{1}, t_{2}, t_{3}\right\rangle$ and $\left\langle t_{1}, t_{3}, t_{2}\right\rangle$, both prefixes are now represented as a single state $\left[t_{1}, t_{2}, t_{3}\right]$. This representation reduces the number of escaping arcs and hence increases precision values. Using multiset state representation and precision calculation as explained in Section 5.3, the model in Figure 5.9 and $\log$ in Table 5.3 used to motivate this section has a precision value of 1 (perfect). It is worth mentioning that in [12], the authors also propose the use of set as state representation. However, this is not applicable to our case: unlike sequence or multiset, a set does not preserve the number of occurrences of each task executed, and therefore, it may represent a (possible infinite) number of different model states. For example, given the model in Figure 5.2, the set $\left\{t_{1}, t_{2}, t_{9}\right\}$ represents $\left\langle t_{1}, t_{2}, t_{9}\right\rangle,\left\langle t_{1}, t_{2}, t_{9}, t_{2}\right\rangle,\left\langle t_{1}, t_{2}, t_{9}, t_{2}, t_{9}\right\rangle, \ldots$

\subsubsection{Abstraction on the Direction}

In the approach presented in Section 5.3, the prefixes of the complete tasks sequences are used to build the automaton. For example, given a complete task sequence $\left\langle t_{1}, t_{2}, t_{3}, t_{4}\right\rangle$, the states constructed from the sequence are the empty sequence \langle\rangle (corresponding with $\left\langle\bullet t_{1}, t_{2}, t_{3}, t_{4}\right\rangle$, where $\bullet$ indicates a point of interest in the sequence), $\left\langle t_{1}\right\rangle$ (for $\left.\left\langle t_{1} \bullet, t_{2}, t_{3}, t_{4}\right\rangle\right)$, $\left\langle t_{1}, t_{2}\right\rangle$ (for $\left.\left\langle t_{1}, t_{2}, t_{3}, t_{4} \bullet t_{3}, t_{4}\right\rangle\right),\left\langle t_{1}, t_{2}, t_{3}\right\rangle$ (for $\left\langle t_{1}, t_{2}, t_{3} \bullet t_{4}\right\rangle$ ) and finally $\left\langle t_{1}, t_{2}, t_{3}, t_{4}\right\rangle$ (for $\left.\left\langle t_{1}, t_{2}, t_{3}, t_{4} \bullet\right\rangle\right)$. In other words, only the activities in the past are used and we move forward on the complete task sequences. This approach is used by all existing precision checking techniques $[62,64,63]$.

In [12], the authors show that any point in the sequence (represented as $\bullet$ ) may represent two complementary visions: the past tasks seen until that point (as it has been shown above), but also the future tasks to come until the ending of the case. For instance, given $\left\langle t_{1}, t_{2} \bullet t_{3}, t_{4}\right\rangle,\left\langle t_{1}, t_{2}\right\rangle$ are the tasks occurred, while $\left\langle t_{3}, t_{4}\right\rangle$ are the tasks to happen. Both $\left\langle t_{1}, t_{2}\right\rangle$ and $\left\langle t_{3}, t_{4}\right\rangle$ are used in [12] as two different states that can be derived from the same point in the sequence. In this section, we use the same idea to present a backward precision measurement, that complements the forward approach presented before. The combination of metric results for both approaches will lead to a measurement unbiased by the direction of the precision checking. For the sake of clarity we will use ordered state representation to illustrate the remainder of the section, although the analogous procedure is applicable for unordered representation.

Let $\Lambda$ be the option chosen to compute precision, i.e., $\Lambda^{1}, \Lambda^{*}$ or $\Lambda^{R}$. In order to build the automaton for the backward precision measurement, we consider the prefixes of the reversed complete tasks sequences in $\operatorname{row}_{T}(\Lambda)$. In other words, given $\operatorname{row}_{T}(\gamma)=\left\langle t_{1}, t_{2}, t_{3}, t_{4}\right\rangle$ of the alignment $\gamma \in \Lambda$, we use $\operatorname{row}_{T}^{\prime}(\gamma)=\left\langle t_{4}, t_{3}, t_{2}, t_{1}\right\rangle$ to determine the states, resulting in the 
following 5 states: \langle\rangle (corresponding with $\left.\left\langle\bullet t_{4}, t_{3}, t_{2}, t_{1}\right\rangle\right),\left\langle t_{4}\right\rangle\left(\right.$ for $\left.\left\langle t_{4} \bullet t_{3}, t_{2}, t_{1}\right\rangle\right),\left\langle t_{4}, t_{3}\right\rangle$ $\left(\right.$ for $\left.\left\langle t_{4}, t_{3} \bullet t_{2}, t_{1}\right\rangle\right),\left\langle t_{4}, t_{3}, t_{2}\right\rangle$ (for $\left.\left\langle t_{4}, t_{3}, t_{2} \bullet t_{1}\right\rangle\right)$ and finally $\left\langle t_{4}, t_{3}, t_{2}, t_{1}\right\rangle$ (for $\left\langle t_{4}, t_{3}, t_{2}, t_{1} \bullet\right\rangle$ ). Analogously, the set of complete tasks sequences of $M$ is also reversed. ${ }^{1}$ The rest of the precision checking is performed as it is described in Section 5.3.

Figure 5.12 shows an example of two automata for the trace $\langle a, b, c, d\rangle$, constructed by moving in forward direction (left) and by moving backward (right). Notice the difference of identified escaping arcs shown by the two automata. Finally, precision values obtained using forward and backward-constructed automaton can be combined (e.g., the average), resulting in a balanced precision metric unbiased by the direction of the automaton constructed. Note that more sophisticated and flexible combinations of both metrics are also possible. In Section 5.5, we investigate the differences in precision values produced by the various approaches using a variety of even logs and models.

\footnotetext{
${ }^{1}$ Notice that, for the case of Petri nets with one unique initial and final markings, the set of all reversed complete transition sequences can be generated by the behavior of a net obtained from the original net by reversing its arcs and swapping their initial with final marking.
} 


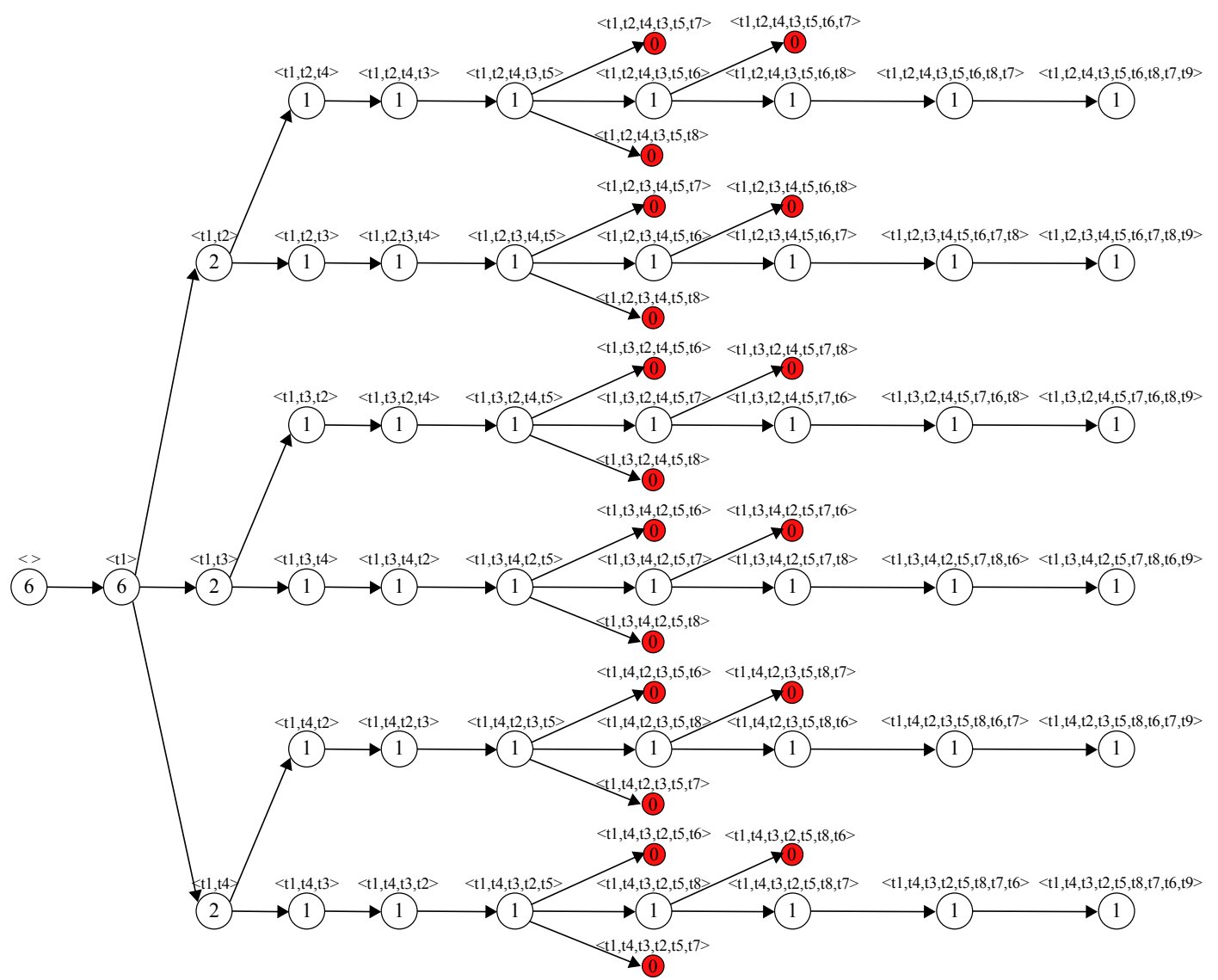

Figure 5.10: Precision automaton and escaping arcs between the model in Figure 5.9 and the log in Table 5.3. 


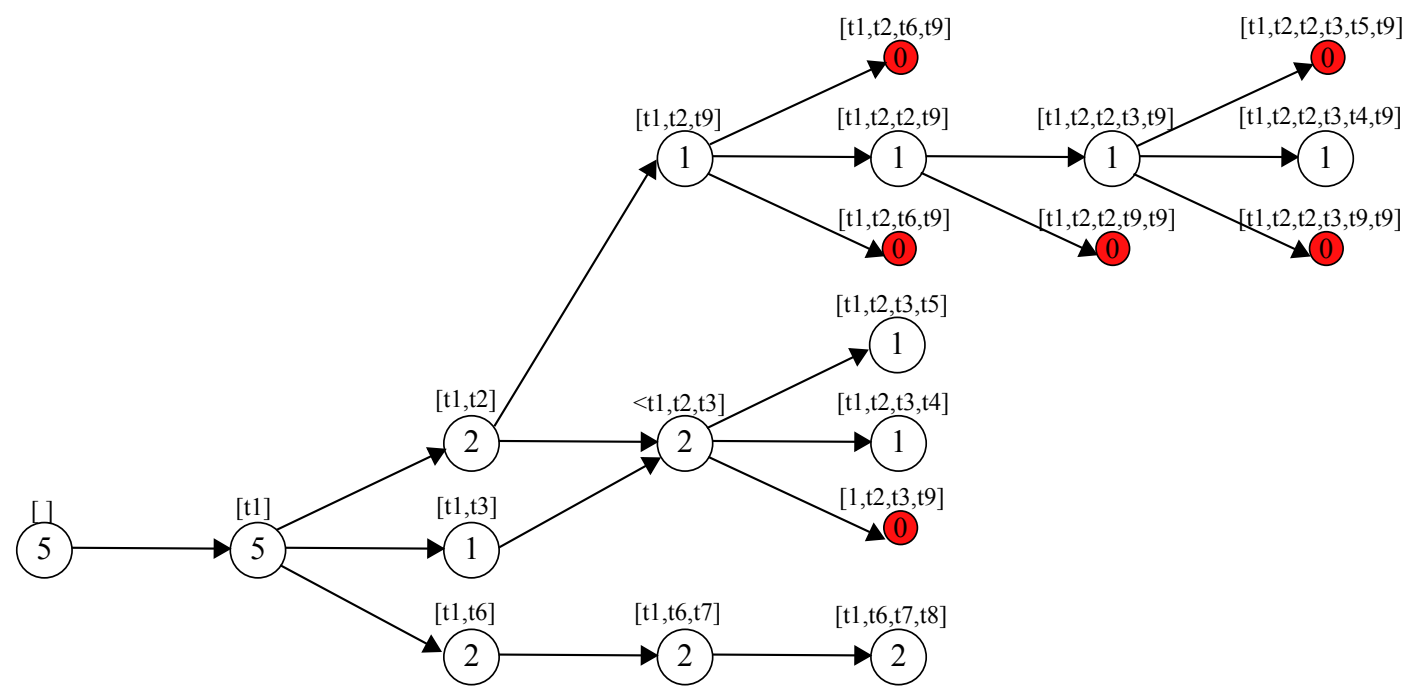

Figure 5.11: Automaton from $\Lambda^{1}$ with multiset state representation for the running example medical process.
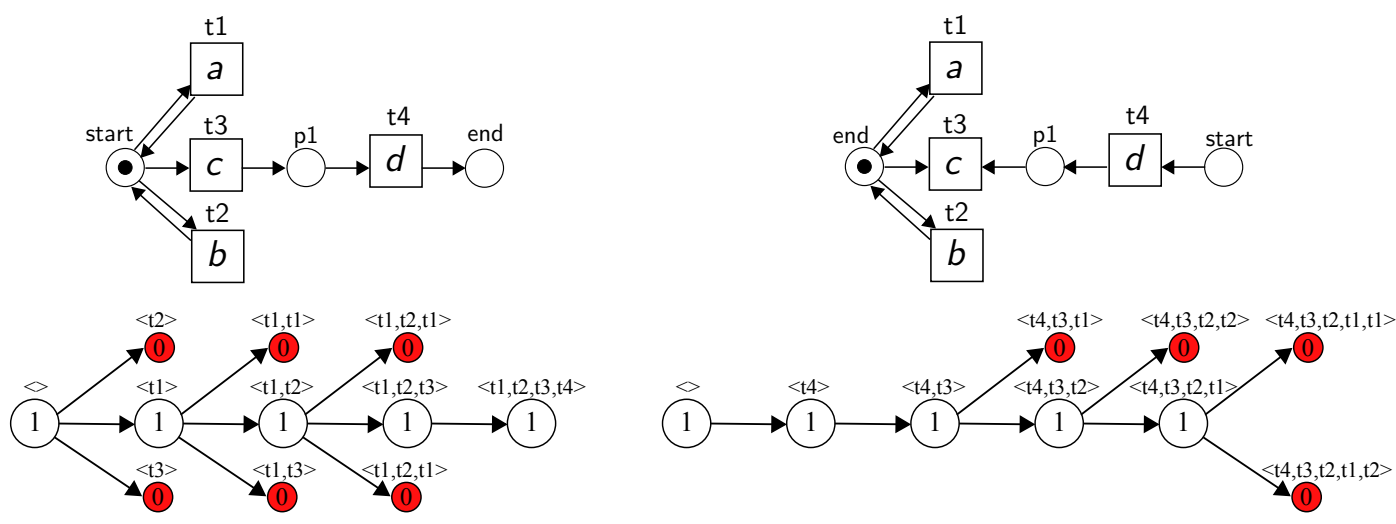

Figure 5.12: Example of model and resulting automaton for the trace $\langle a, b, c, d\rangle$, with both forward and backwards approaches. 


\subsection{Experimental Results}

In this section we illustrate experimentally some of the concepts presented in the chapter.

The first set of experiments was performed to evaluate the precision measurements provided by the proposed metrics. In particular, we measured whether the proposed precision metrics are unidimensional [95], i.e., not sensitive to non-fittingness of event logs. We measured precision between various logs and models whose expected values are known. Furthermore, we compared the values obtained against existing state-of-the-art metrics for precision: etc $c_{P}$ [62] (presented in Chapter 3), behavioral precision [97], and weighted behavioral precision $[27]$.

By combining completely precise and flower models and log in various ways, we created new models whose expected precision values are between the two extremes. Two models were combined by merging the end place of one with the initially marked place of another. The merged models were named according to the name of their original models, e.g., PF model is the result of merging the end place of completely precise $\mathbf{P}$ model with the initially marked place of the flower model $\mathbf{F}$. Precision values were measured 30 times using 30 event logs, each consists of 5,000 traces, generated by simulating the precise model (i.e., PP). For sake of completeness, we also measured the precision of the overfitting model $(\mathbf{P})$ and the flower model (F) using 30 logs of 5,000 traces generated by simulating the $\mathbf{P}$ model. This way, each log contains all the possible behavior of the model that generates it (i.e., all directly follow relations between two activities that are allowed according to the model are recorded in the $\log$ ).

The top part of Figure 5.13 shows the alignment-based precision values, measured using all optimal alignments per trace of the logs. The experiment with one and representative alignments per trace yields identical results. This result shows that by observing sufficiently enough behavior in the event logs, all alignment-based metrics provide similar intuition about precision of models, i.e., overfitting models have high precision values and "flower" models have low precision values. Note that there are slight differences between various configurations of metrics, i.e., states (ordered/unordered) and forward/backward constructed automata.

To evaluate the robustness of the metrics against non-fitting logs, we took the models and logs from the previous experiments and created unfitting logs by removing $n$ random events per trace from the fitting logs. Furthermore, the measurements are compared against existing metrics. We use the CoBeFra tool [28] to measure behavioral precision [97] and weighted behavioral precision [27]) and use ProM 6 to measure $e t c_{P}$. The bottom part of Figure 5.13, Figures 5.14 and 5.15 show some of the results.

The bottom part of Figure 5.13 shows that the metrics proposed in this chapter are robust to fitness problems. Even in cases where almost half of the events in all traces are removed, all alignment-based metrics provide similar value as the ones provided for perfectly fitting traces. Figure 5.14 shows a comparison between the precision values provided by alignmentbased metrics and other existing metrics. For readability, we only show one alignment-based 


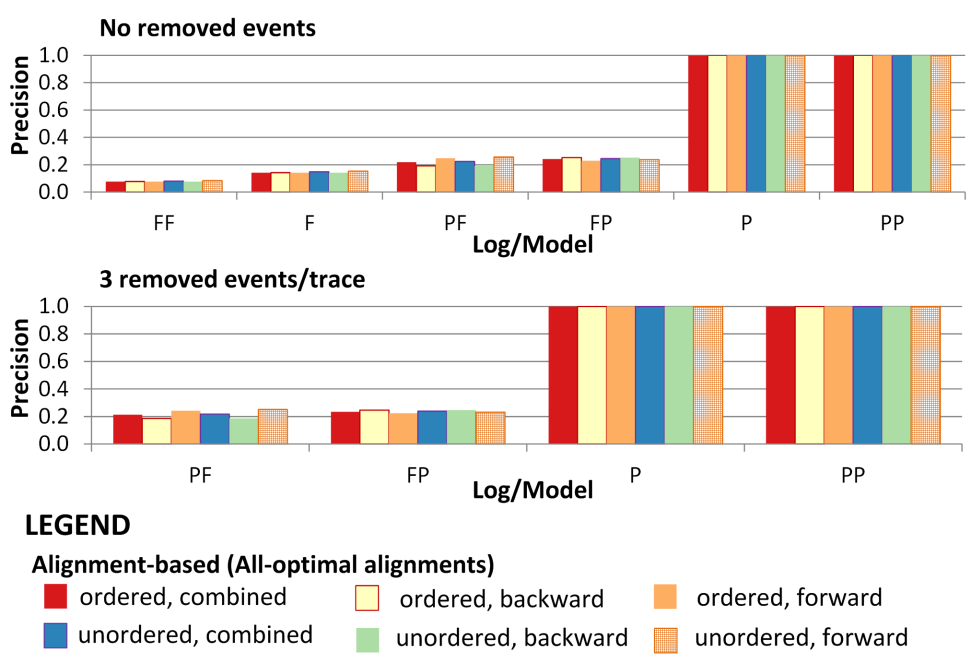

Figure 5.13: Precision values of flower and over-precise logs/models and their combinations provided by alignment-based approach (i.e., computed using all optimal alignments, ordered, and forward-constructed automata). If all behavior are observed in the original logs, all measurements are insensitive to non-fitting traces.

metric: the one computed using all-optimal alignments and forward-constructed automata whose states are constructed by taking into account activity ordering. Note that in cases where logs are perfectly fitting the models, all metrics provide similar precision intuition. In fact, the alignment-based precision values shown in Figure 5.14 are the same as the etc $_{P}$ values. However, in cases where logs are non-fitting, other metrics may show misleading precision insights. The $e t c_{P}$ metric provides low precision for model $\mathbf{P F}$ with respect to perfectly fitting $\log$ (i.e., 0.25 ). However, the value rises to 0.82 when 3 events are removed from the logs, because for all non-fitting traces it ignores the rest of the traces after the first non-fitting event occur. Similarly, both weighted and unweighted behavioral precision metrics provide lower precision values for non-fitting logs than the ones provided for perfectly fitting logs. Even for overly fitting models $\mathbf{P}$ and $\mathbf{P P}$, both metrics provide precision values below half (i.e., indicating the models are imprecise). This occurs because both metrics mixed both perfectly-fitting and non-fitting traces in construction of artificial negative events, which leads to misleading construction of artificial negative events.

Figure 5.15 shows the influence of noise by removing some events in the logs. As shown in the figure, other than the alignment-based precision metric, precision values of all metrics may change significantly even with only one event removed from all traces. Due to the randomness of the location of removed events, the $e t c_{P}$ metric may both increases or decreases with the presence of non-fitting traces. Both weighted and unweighted behavioral precision 


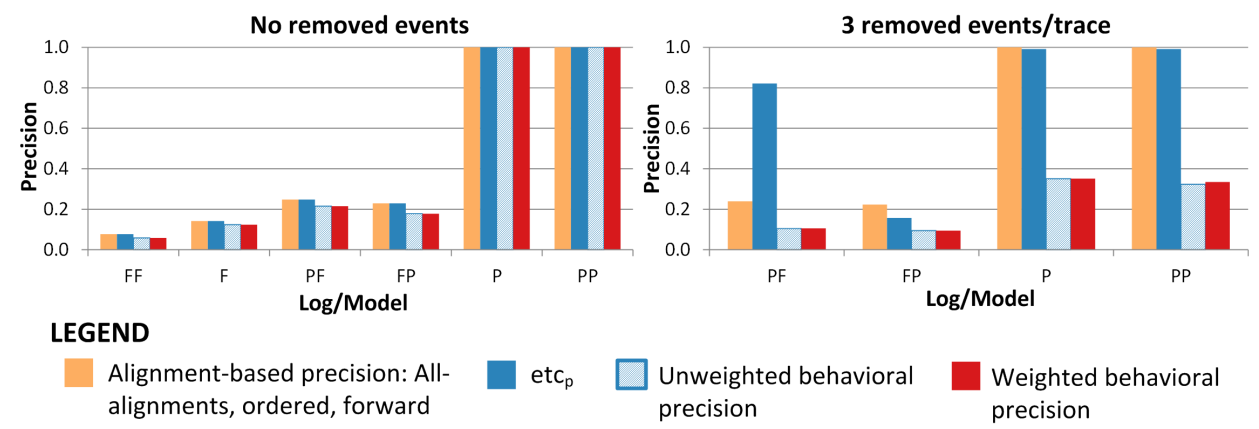

Figure 5.14: Comparison between precision values obtained using alignment-based approach (i.e., computed using all optimal alignments, ordered, and forward-constructed automata) and other metrics. Only the alignment-based approach is not sensitive to non-fitting $\operatorname{logs} /$ models.

metrics decreases when more events are removed because incorrect artificial negative events are introduced. Note that the number of negative events tends to decrease when traces in the log gets more vary because of the removal of events.
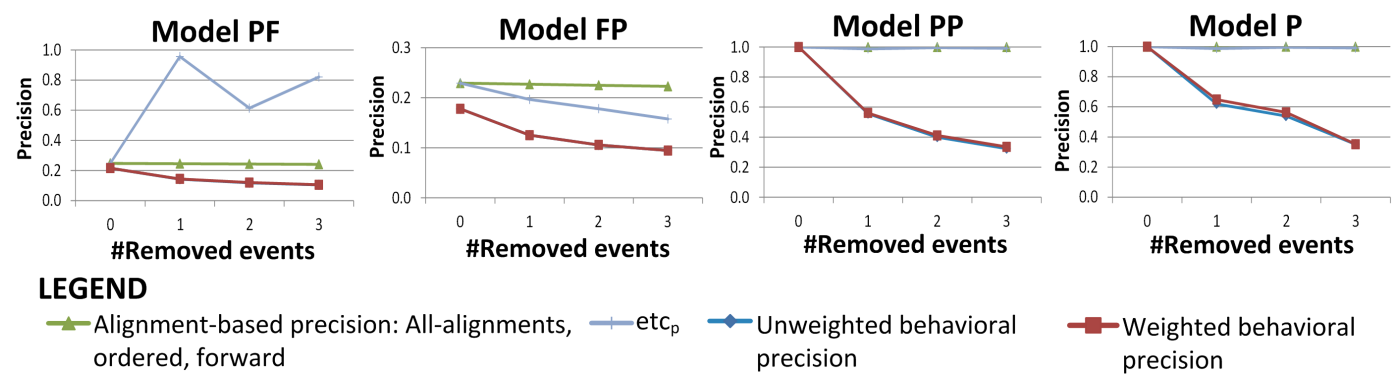

LEGEND

-Alignment-based precision: All-alignments, ordered, forward

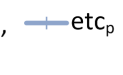
$\neg$ Unweighted behavioral precision precision

Figure 5.15: Precision values of different metrics for perfectly fitting logs and non-fitting logs created by removing some events in the logs. Only the alignment-based approach metric (i.e., computed using all optimal alignments, ordered, and forward-constructed automata) is insensitive to non-fitting logs.

The set of experiments also shows some interesting insights into differences between alignment-based metrics. Figure 5.16 shows a comparison between precision values provided by the two metrics for models PF and FP. As shown in the figure, precision values of alignment-based metrics provided by forward-constructed automata for model $\mathbf{P F}$ is higher than the values provided by backward-constructed automata for the same model, regardless of the noise level and the state representation (ordered/unordered). In contrast, the values 
provided by the latter is higher than the former for the FP model. This shows that the position of the precise part of the models influences precision values. Precision values are higher when the direction of constructed automata starts with precise part of process models. In this case, we clearly see the influence of forward/backward direction of constructed automata to precision values. To balance the influence, one of the simplest way is to take the average between the values provided by both directions. Figure 5.16 shows that the precision values obtained by combining both values are almost similar between model $\mathbf{P F}$ and $\mathbf{F P}$.
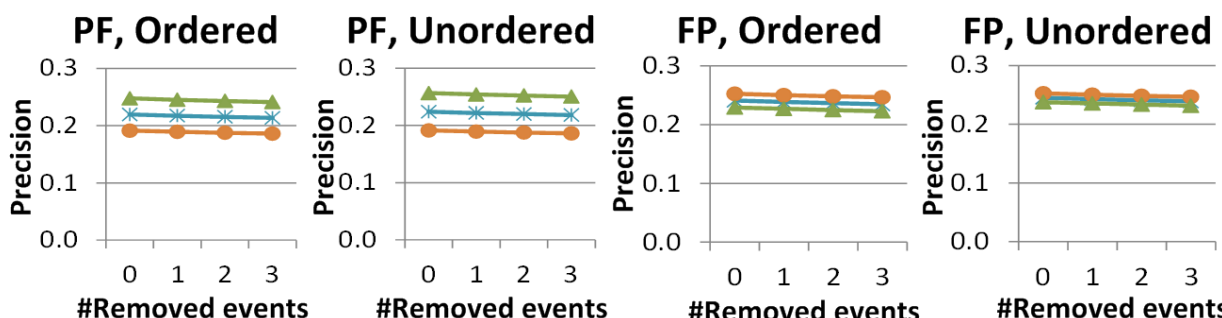

LEGEND

$*$ Combined

$\rightarrow$ Forward

Backward

Figure 5.16: Precision values of the PF and FP using all-alignments per trace, with different state representations (ordered/non-ordered) and direction (forward/backward). Higher precision is obtained when the direction of automata construction starts with precise part of the models.

To evaluate the applicability of the approach to handle real life logs, we used 8 pairs of process models and logs from two different domains (see Table 5.4), where 7 logs and models were obtained from municipalities in the Netherlands. In particular, we took the collections of logs and models from the CoSeLoG project [34]. The remaining pair of log and model is obtained from a hospital in the Netherlands ${ }^{2}$. The logs and models from municipalities are related to different types of building permission applications, while the hospital log is related to patient handling procedure. All processes have unlogged tasks, and some of the models allow loops. Table 5.4 shows an overview of the logs and models used in the experiments. \#Deviations/trace column indicates the number of asynchronous moves after aligning all traces in the logs with their corresponding models. As shown in Table 5.4, all logs are not perfectly fitting to the corresponding models. We measure the precision values for all $\operatorname{logs}$ and the computation time required. The results are shown in Figure 5.17 and Figure 5.18.

Figure 5.17 reports precision values obtained for real-life logs and models. Only the approach based on 1-alignment provides precision values for all real-life logs and models in the experiments. The approach based on all-optimal alignments per trace had out-of-memory problems when dealing with relatively complex process models and logs such as "Bouw-1" (33 places, 34 transitions), "Bouw-4" (31 places, 31 transitions), and "MLog-3" (24 places,

\footnotetext{
${ }^{2}$ see http://www.healthcare-analytics-process-mining.org/
} 


\begin{tabular}{|r|r|r|r|r|r|}
\hline Log & \multirow{2}{*}{ \#Cases } & \#Events & \multicolumn{2}{|c|}{ Process Model } & \#Deviation/trace \\
\cline { 4 - 5 } & & & \#Place & \#Trans & \\
\hline Bouw-1 & 139 & 3,364 & 33 & 34 & 9.75 \\
\hline Bouw-4 & 109 & 2,331 & 31 & 31 & 7.27 \\
\hline MLog1 & 3,181 & 20,491 & 15 & 12 & 5.33 \\
\hline MLog2 & 1,861 & 15,708 & 16 & 19 & 1.45 \\
\hline MLog3 & 10,271 & 85,548 & 24 & 21 & 14.50 \\
\hline MLog4 & 4,852 & 29,737 & 16 & 27 & 2.09 \\
\hline MLog5 & 25,846 & 141,755 & 14 & 24 & 1.21 \\
\hline IsalaLog & 77 & 459 & 26 & 39 & 0.68 \\
\hline
\end{tabular}

Table 5.4: Real-life logs and models used for experiments
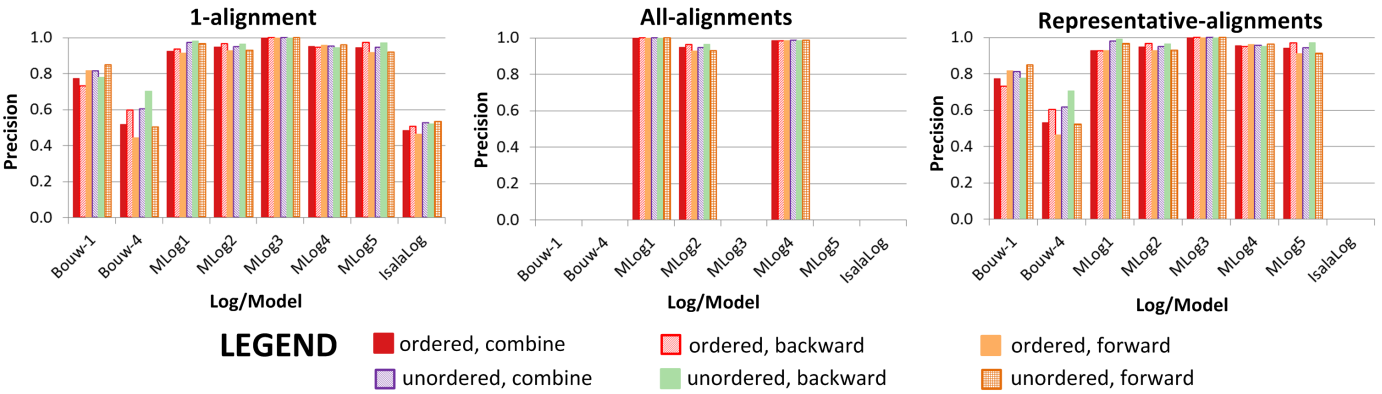

Figure 5.17: Precision values of real-life logs and models. Only the 1-alignment approach manages to provide precision results for all logs/models.

21 transitions). Precision measurements based on representative of optimal alignments also had the same problems dealing with the hospital log (i.e., "IsalaLog"). Although the model of the log is relatively small, it contains many unlogged tasks (tasks whose execution are not logged), allows loops, and allow many interleaving activities such that the size of state space required to compute even representative of all optimal alignments is large and does not fit memory.

Nevertheless, notice the similarity of the computed precision values using all three alignments (1-align, all-align, and representatives). From all pairs of logs and models, only 2 of them have precision value below 0.7 . This shows that in reality, process models are made to be relatively precise such that meaningful insights into the process can be obtained. Interestingly, different precision values are provided by different metrics in the experiment with log and model "Bouw-4" when both one and representative alignments are used. The precision value provided by ordered-forward metric for the model is around 0.44 (showing imprecision) while the unordered-backward precision metric provides a value of 0.7 (i.e., precise). This indicates that more observations are required to measure the particular log and model accurately.

Figure 5.18 reports the computation time required to measure precision of real-life logs 

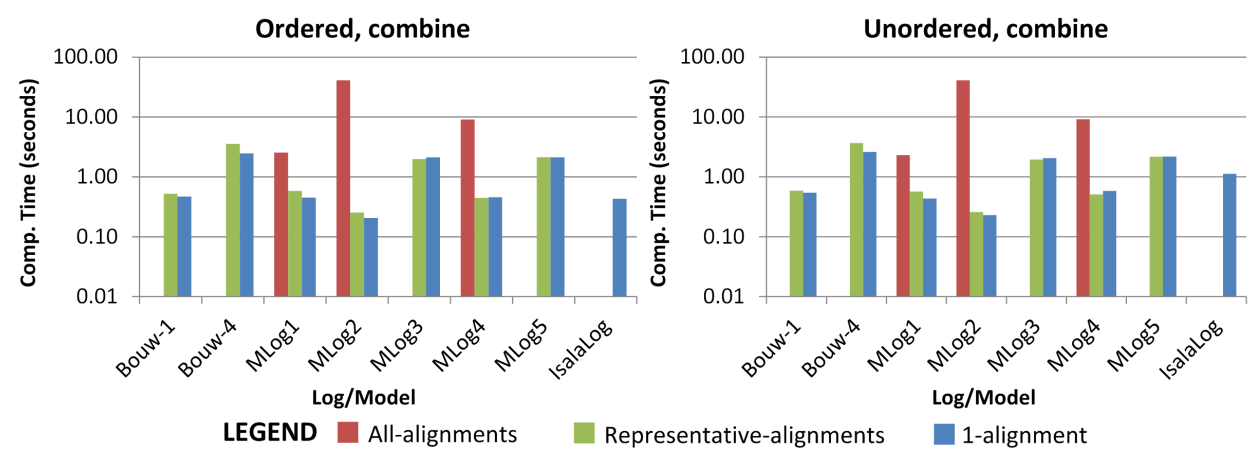

Figure 5.18: Computation time comparison of alignment-based precision measurement using combined values (from backward and forward automata construction). Y-axis values are shown in a logarithmic scale.

and models using alignment-based approach with combined precision values between forward and backward-constructed automata. The y-axis of the charts are shown in logarithmic scale. As shown in the figure, the computation time of precision measurement with all-alignments takes much longer than the ones required by one or representative alignments. All measurements using 1-alignment/representative alignments were computed in less than 10 seconds. Notice the similarity between the left and right graph on the figure (except the IsalaLog that has out-of-memory problem in the approach with representative alignments). In fact, we obtained identical results for all other combination of state representations (ordered/unordered) and directions where automata is constructed (forward/backward). This shows that the different directions of the automata construction and state representations are not significantly influencing computation time. Instead, most computation time of precision measurement is spent in the alignment of logs and process models. Another interesting observation is that the time spent to compute representative alignments are similar to the time spent to compute 1-alignment. Thus, we recorded the number of generated representatives for the experiments and other statistics to investigate this.

\subsection{Conclusions}

The direct use of event logs to determine the observed behavior is susceptible to get misaligned when it is compared with the modeled behavior, i.e., situations where the model is unable to determine the system state given the observed behavior. This chapter proposes the use of aligning approaches as a previous step before the precision computation. In particular, the chapter extends the escaping arcs theory to use the 1-alignment, all-alignment and representative-alignment to determine the observed behavior, instead of using directly 
the log. Furthermore, the chapter proposes an abstraction of the escaping arcs approaches, where different state representations and different automaton construction are considered. 


\section{Part III}

\section{Decomposition in Conformance Checking}





\section{Chapter 6}

\section{Decomposed Conformance Checking}

\subsection{Introduction}

While in the previous chapters we analyzed the precision dimension, in these second part we focus on the fitness dimension of conformance checking. In real-life situations, event logs often do not fit its corresponding models, i.e., some log traces cannot be fully reproduced in the model. These non-fitting situations should be communicated to the stakeholders, in order to take decisions on the process object of study. However, in reality process models can be non-deterministic, which complicates the analysis. Non-determinism may arise when the model contains silent or duplicate activities, which is often the case in practice. Moreover, the presence of noise in the log - e.g., incorrectly logged events or abnormal behavior complicates even more the algorithmic detection of non-fitting situations. Due to this, the initial fitness approaches based on replaying log traces in a model in order to assess whether a trace can fit a model [80] have been recently reconsidered, giving rise to approaches based on alignment [16]. Alignment techniques relate complete firing sequences of the model and traces in the event log. The techniques can cope with deviations and models with duplicate/invisible activities. However, alignment techniques are extremely challenging from a computational point of view. Traces in the event log need to be mapped on paths in the model. A model may have infinitely many paths and the traces may have an arbitrary amount of deviating events. Hence, although the algorithms have demonstrated to be of great value for undertaking small or medium-sized problem instances [4, 49], they are often unable to handle problems

\footnotetext{
- The materials reported in this chapter are partially published in [67] and [65]-
} 
of industrial size. Decomposition techniques are an important means to tackle much large and more complex process mining problems. Therefore, this chapter addresses this problem through decomposition and distribution, using Petri nets as the modeling class (although the conclusions can be extrapolated to other process model notations).

There is a trivial way to decompose the fitness checking problem. One can simply split the event log into sublogs such that every trace appears in precisely one of these sublogs. Note that the conformance is still checked on the whole model. Linear speed-ups are possible using such a simple decomposition. However, the real complexity is in the size of the model and the number of different activities in the event $\log [15]$. Therefore, we propose a different approach. Instead of trying to assess the fitness of the whole event log and the complete Petri net, fitness checking is only performed for selected subprocesses (subnets of the initial Petri net and corresponding sublogs). Subprocesses are identified as subnets of the Petri net that have a single-entry and a single-exit node $(S E S E)$, thus representing an isolated part of the model with a well-defined interface to the rest of the net. SESEs can be efficiently computed and hierarchically represented in a tree-like manner into the Refined Process Structured Tree (RPST) [73].

The remainder of this chapter is organized as follows. Related work and other approaches for decomposing conformance checking are presented in Section 6.2. Section 6.3 presents the definitions of Single-Entry Single-Exit and Refined Process Structured Tree. The decomposition of a Petri net in terms of SESEs is presented in Section 6.4, and Section 6.5 defines a valid decomposition where the conformance properties are preserved. In Section 6.6 we provide some experimental results. Finally, Section 6.7 concludes the chapter.

\subsection{Related Work}

Decomposing large graphs into smaller fragments is a topic widely studied in the literature. There exist plenty of techniques with different goals for that task, for example, minimizing the connectivity among fragments [52]. In particular, this chapter aims to obtain fragments with a single entry node and a single exit node [72]. SESEs, presented in Section 6.3, capture the idea of subprocess with a clear entry and exit point within the main process. In [93, 94] the authors proposed a Refined Process Structure Tree, a tree structure of SESEs. The proposed RPST has some desirable properties: it is unique, it is modular, and can be computer in linear time. The linear time computation is based on the idea of triconnected components in [91]. The original RPST algorithm [93] contained a post-processing step fairly complex. In [72, 73 the computation of this post-processing step is considerably simplified by introducing a pre-processing step that splits every node with more than one incoming andmore than one outgoing edge into two nodes. Both the theory and the implementation used in this chapter is based on $[72,73]$

Decomposition techniques have been applied recently in process mining. In [2], the author proposes a new decomposition concept: passages. A passage is a pair of two non-empty sets of activities $(X, Y)$ such that the set of direct successors of $X$ is $Y$ and the set of direct 
predecessors of $Y$ is $X[2]$. Process discovery and conformance checking can be done at a passage level and the results can be aggregated. This has advantages in terms of efficiency and diagnostics. Moreover, passages can be used to distribute process mining problems over a network of computers.

In [1] the author formalizes the concept of valid decomposition to decompose in a generic way process discovery and conformance problems. A decomposition is valid, presented in detail in Section 6.5, if the resulting subnets "agree" on the original labeling function, each place resides in just one subnet, and also each invisible transition resides in just one subnet. Moreover, if there are multiple transitions with the same label, they should reside in the same subnet. Only unique visible transitions can be shared among different subnets.

Finally, conformance checking and decomposition are also considered in the work on proclets [45]. Proclets can be used to define the so-called artifact centric processes, i.e., processes that are not monolithic but that are composed of smaller interacting processes (called proclets). In [45] it is shown that conformance checking can be done per proclet by projecting the event log onto a single proclet while considering interface transitions in the surrounding proclets.

\subsection{Single-Entry Single-Exit and Refined Process Struc- ture Tree}

The intuitive idea behind the decomposition technique in this chapter is to find subgraphs that have a simple interface with respect to the rest of the net. The following set of definitions formalize the idea of Single-Entry Single-Exit subnet and the corresponding decomposition. The underlying theory dates back to the seminal work of Hopcroft and Tarjan in the seventies [48], but recent studies have made considerable progress into making the algorithms practical when applied to process models [73, 72]. We start defining the graph structure used for decomposing a process model: the workflow graph. Given a workflow net ${ }^{1}$, the workflow graph represents the arcs between the nodes of the net.

Definition 6.1 (Workflow Graph). Let $W N=(P, T, F$, start, end) be a workflow net, where $P$ are the places, $T$ are the transitions, and $F$ are the arcs. We define the workflow graph of $W N$ simply as the directed graph $G(W N)=(N, F)$ where no distinctions are made between places and transitions, i.e., $N=P \cup T$ represent the nodes of the graph, and $F$ the arcs. We can omit the parameter $W N$ whenever the context is clear.

An example of workflow graph Figure $6.1 \mathrm{~b}$ (only the graph but not the squares) is shown for the workflow net in Figure 6.1a.

\footnotetext{
${ }^{1}$ Although the approach presented in this chapter can be generalized to general Petri nets with several sources and sinks, for the sake of clarity in this paper we restrict to the workflow case with only one source and only one sink [73].
} 
(a)

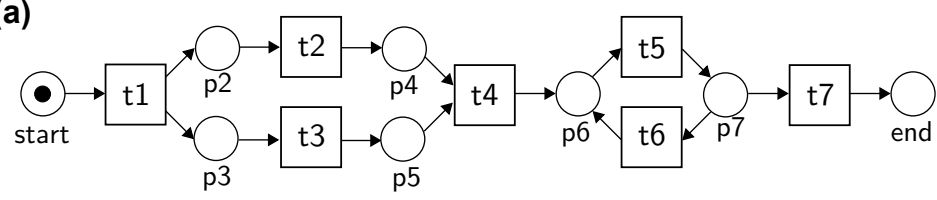

(b)

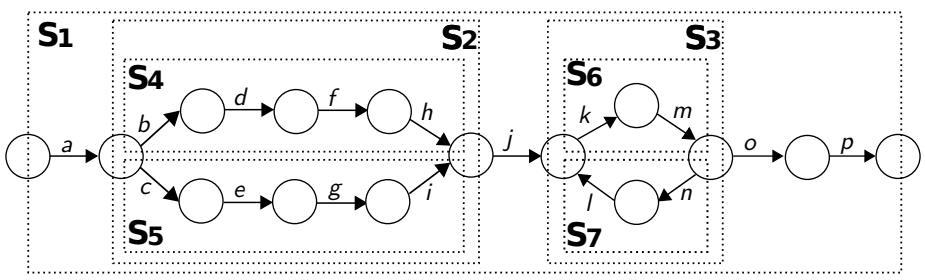

(c)

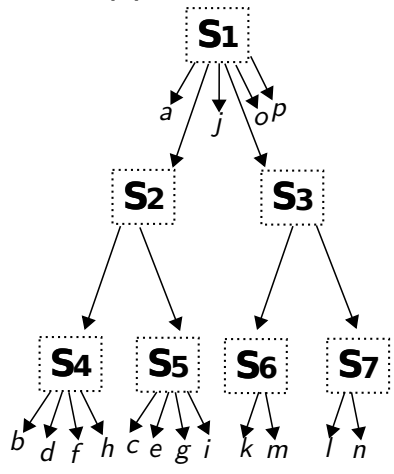

Figure 6.1: An example of (a) workflow net, (b) its workflow graph, and the (c) RPST with its SESE decomposition.

In the remainder, the following context is assumed: Let $G$ be the workflow graph of a given $W N$, and let $G_{S}=\left(V_{S}, S\right)$ be a connected subgraph of $G$ formed by a set of edges $S$ and the vertices $V_{S}=\Pi(S)$ induced by $S .^{2}$

Definition 6.2 (Subnet nodes [73]). A node $x \in V_{S}$ is interior with respect to $G_{S}$ iff it is connected only to nodes in $V_{S}$; otherwise $x$ is a boundary node of $G_{S}$. A boundary node $y$ of $G_{S}$ is an entry of $G_{S}$ iff no incoming edge of $y$ belongs to $S$ or if all outgoing edges of $y$ belong to $S$. A boundary node $y$ of $G_{S}$ is an exit of $G_{S}$ iff no outgoing edge of $y$ belongs to $S$ or if all incoming edges of $y$ belong to $S$.

For example, let us consider the $\operatorname{arcs} S_{4}=\{b, d, f, h\}$ of Figure 6.1, and the set of vertices induced by them $V_{S_{4}}=\left\{t_{1}, p_{2}, t_{2}, p_{4}, t_{4}\right\} . t_{1}$ and $t_{4}$ are boundary nodes, while $p_{2}, t_{2}, p_{4}$ are interior. Moreover, $t_{1}$ is an entry, while $t_{4}$ is an exit.

As next definition formalizes, a SESE is a special type of subgraph with a very restricted interface with respect to the rest of the graph:

Definition 6.3 (Single-Exit-Single-Entry [73]). A set of edges $S \subseteq F$ is a SESE (SingleExit-Single-Entry) of graph $G=(N, F)$ iff $G_{S}$ has exactly two boundary nodes: one entry and one exit. A SESE is trivial if it is composed of a single edge. $S$ is a canonical SESE of $G$ if it does not partially overlap with any other SESE of $G$, i.e., given any other SESE $S^{\prime}$ of $G$, they are nested $\left(S \subseteq S^{\prime}\right.$ or $\left.S^{\prime} \subseteq S\right)$ or they are disjoint $\left(S \cap S^{\prime}=\emptyset\right)$. By definition, the source start of a workflow net is an entry to every fragment it belongs to and the sink end of the net is an exit from every fragment it belongs to.

\footnotetext{
${ }^{2} \Pi(R)=\bigcup_{(a, b) \in R}\{a, b\}$ is the set of elements referred to by relation $X \subseteq A \times B$.
} 
The decomposition based on canonical SESEs is a well studied problem in the literature, and can be computed in linear time. In [94], the authors proposed the algorithm for constructing the RPST, i.e., a hierarchical structure containing all the canonical SESEs of a model. In [73], the computation of the RPST is considerably simplified and generalized by introducing a pre-processing step that reduces the implementation effort considerably.

Definition 6.4 (Refined Process Structured Tree [73]). Let $G$ be the workflow graph of a given workflow net. The Refined Process Structured Tree (RPST) of $G$ is the tree composed by the set of all its canonical SESEs, such that, the parent of a canonical SESE $S$ is the smallest canonical SESE that contains $S$. The root of the tree is the entire graph, and the leaves are the trivial SESEs. The set of all the nodes of the tree is denoted as $\mathbb{S}$.

Figure 6.1 show the RPST and the canonical SESEs in the example of the same figure. In the remainder of the chapter, we will refer to canonical SESEs resulting from the RPST decomposition simply as SESEs. Also note that the SESEs are defined as a set of edges (i.e., $S$ ) over the workflow graph (not as subgraphs, i.e., $G_{S}$ ). However, for simplicity and when the context is clear, we will use the term SESE to refer also to the subgraph induced by those edges. We will denote as $P N^{S}=\left(P^{S}, T^{S}, F^{S}\right)$ the Petri net determined by the SESE $S$, i.e., $P N^{S}=(P \cap \Pi(S), T \cap \Pi(S), F \cap S)$. The nodes (either transitions or places) determined by $S$ are denoted as $N^{S}$, i.e., $(P \cup T) \cap \Pi(S)$.

\subsection{Decomposing Conformance Checking using SESEs}

It is well known that checking conformance of large logs and models is a challenging problem. The size of $\log$ and model and the complexity of the underlying process strongly influence the time needed to compute fitness and to create optimal alignments. Divide-and-conquer strategies are a way to address this problem $[66,65,67,1]$. As indicated before, we do not just want to partition the traces in the event log (providing a trivial way to distribute conformance checking). The potential gains are much higher if also the model is decomposed and traces are split into smaller ones. To decompose conformance checking problems, the overall system net $S N$ is broken down into a collection of subnets $\left\{S N^{1}, S N^{2}, \ldots, S N^{n}\right\}$ such that the union of these subnets yields the original system net.

Definition 6.5 (Decomposition). Let $S N=\left(S N M_{I}, M_{F}\right)$ be a system net where $W N=$ $(P, T, F$, start, end $) . D=\left\{S N^{1}, S N^{2}, \ldots S N^{n}\right\}$ is a decomposition of $S N$ if and only if:

- $P=\bigcup_{1 \leq i \leq n} P^{i}$,

- $T=\bigcup_{1 \leq i \leq n} T^{i}$,

- $F=\bigcup_{1 \leq i \leq n} F^{i}$ where $F^{i} \cap F^{j}=\emptyset$ for $1 \leq i<j \leq n$. 
Note that each place or transition can be shared among different subnets, while each arc resides in just one subnet.

Any decomposition that satisfies Definition 6.5 may be considered for decomposing a conformance problem, e.g., subnets containing only one arc, or subnets randomly grouping distant nodes on the net. However, given that the ultimate goal of a decomposition is to be able to diagnose, comprehend and understand conformance problems, the use of meaningful decompositions is preferred, i.e., SESEs. Given the structure of a SESE where a unique single entry and a unique single exit exist, a SESE becomes an appropriate unit of decomposition. Intuitively, each SESE may represent a subprocess within the main process (i.e., the interior nodes are not connected with the rest of the net), and the analysis of every SESE can be performed independently. The RPST of a net can then be used to select a possible set of SESEs forming a decomposition. As it shown in Proposition. 6.6, any transverse cut over the RPST defines a decomposition.

Proposition 6.6 (SESE decomposition). Let $S N=\left(S N M_{I}, M_{F}\right)$ be the system net of the workflow net $W N=(P, T, F$, start, end $)$. Consider the RPST decomposition of $W N$, where $\mathbb{S}$ represents all the SESEs in the RPST. We define a transverse-cut over the RPST as a set of $S E S E s \mathbb{D} \subseteq \mathbb{S}$ such that any path from the root to a leaf of RPST contains one and only one $S E S E$ in $\mathbb{D}$. Given a transverse-cut $\mathbb{D}=\left\{S_{1}, S_{2}, \ldots S_{n}\right\}$, let the decomposition $D_{\mathbb{D}}$ be defined as $D_{\mathbb{D}}=\left\{S N^{S_{1}}, S N^{S_{2}}, \ldots S N^{S_{n}}\right\}$, where $S N^{S_{i}}=\left(P N^{S_{i}}, M_{I} \uparrow_{P} S_{i}, M_{F} \uparrow_{P} S_{i}\right)$, i.e., the Petri net determined by the SESE $S_{i}$, and the projection of the initial and final markings on the places of the subnet. The decomposition $D_{\mathbb{D}}$ derived from the SESEs satisfies the definition of decomposition given in Definition 6.5

Proof. By definition of RPST, the arcs of each SESE in the RPST are contained in one, and only one, of its children (unless it is a trivial SESE). Therefore, any transverse-cut set of SESEs contains all the arcs, where each arc only appears in only one SESE.

Proposition 6.7 (A SESE decomposition from RPST exists). Given any RPST, a decomposition always exists.

Proof. Given any RPST, the root (i.e., the whole net) defines a decomposition. In addition, the set of all the leaves (i.e., the trivial SESEs with only one arc) also defines a decomposition.

As it is claimed in Proposition 6.7, the overall net is, by definition, a decomposition by itself. But it is obvious to see that this trivial way of decomposition does not alleviate the initial conformance problem. On the other hand, a decomposition formed only by trivial SESEs will produce meaningless components, and at the same time, the posterior analysis will have to deal with the analysis overhead produced by the creation of the numerous components. A decomposition which lays in between the aforementioned extremes seems more interesting from the practical point of view, i.e., to generate components large enough to become meaningful subprocesses, but whose size can be handled in practice. Hence, 
the algorithm proposed in Algorithm 6.1 can generate a decomposition which limits the maximum size of each component to $k$ in order to control the size and complexity of individual components.

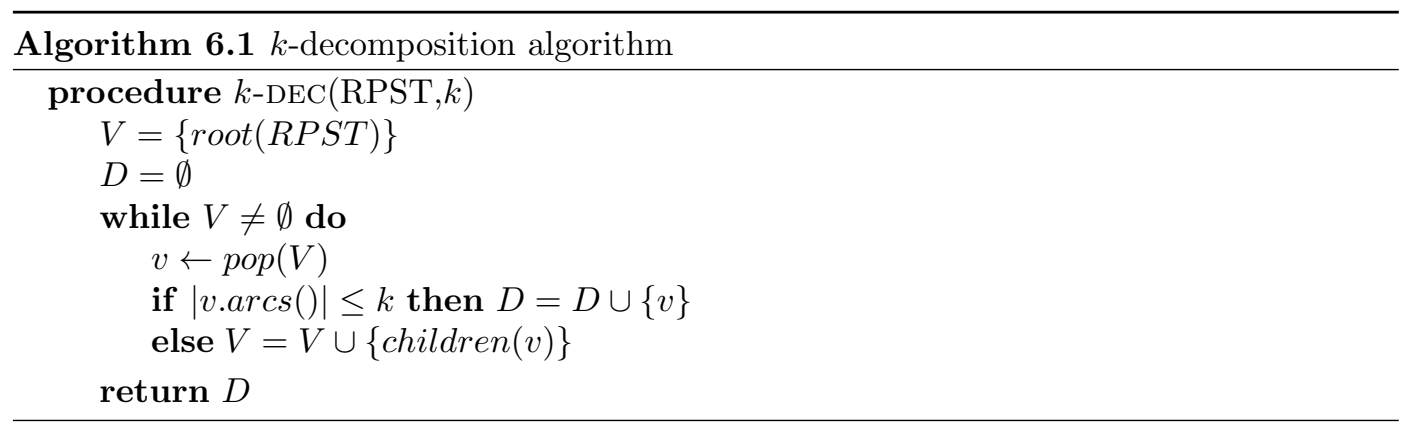

Algorithm 6.1 shows how to compute a $k$-decomposition, for any $k$ such that $1 \leq k \leq|F|$ (where $|F|$ stands for the number of arcs of the overall net). The algorithm keeps a set of nodes that conform the decomposition $(D)$ and a set of nodes to consider $(V)$. Initially $V$ contains the root of the RPST, i.e., the overall net. Then, the algorithm checks, for each node $v$ to consider, if $v$ satisfies the $k$ property, i.e., the number of arcs of SESE $v$ is less or equal than $k$. If this is the case, $v$ is included in the decomposition. If not, it discards $v$ and includes the RPST children of $v$ into the nodes to consider. Note that, given any RPST, a $k$-decomposition always exists, i.e., in worst case, the decomposition formed by all the leaves of the RPST will satisfy the definition. The algorithm proposed has linear complexity with respect to the size of the RPST, and termination is guaranteed by the fact that the size of the component is reduced in every iteration.

\subsection{Bridging a Valid Decomposition}

A SESE is a component that only interfaces with the rest of the net through the single entry and single exit boundary nodes, which may be shared among different components. The rest of nodes of a SESE (i.e., the interior nodes) have no connection with other components. Given that the SESE computation is performed over the workflow graph (i.e., where there is no distinction between places and transitions), we distinguish two possible cases for the boundary nodes: transition boundary and place boundary.

The transition boundary case occurs when the node determined to be the entry or the exit of a SESE is a transition. Figure 6.2 shows an example of a transition boundary. In the example, the overall net is decomposed into two subnets that correspond to the SESEs $S_{1}$ and $S_{2}$, being $d$ the boundary transition shared between them.

As it is proven in [1], a transition boundary decomposition represents no problem from a conformance point of view, i.e., given a decomposition with only transition boundaries, a 


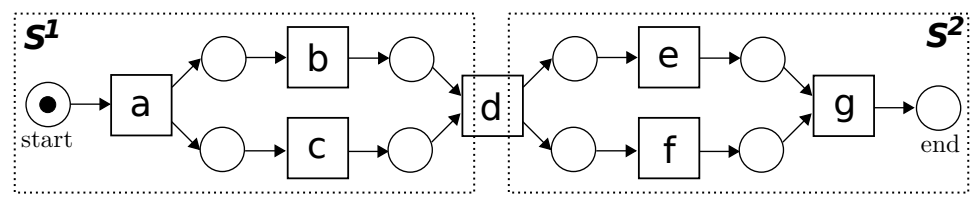

(a) original model

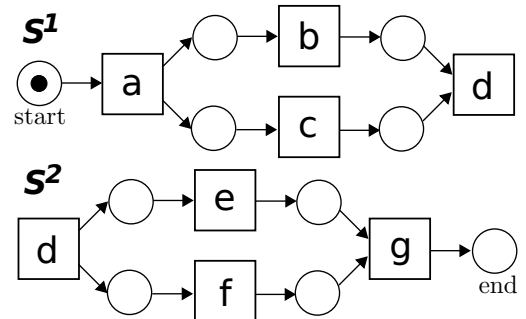

(b) decomposition

$$
\begin{aligned}
& \sigma_{1}=a b c d d e f g \\
& \sigma_{1} \mid \mathbf{s}^{1}=a b c d d \\
& \sigma_{1} \mid \mathbf{s}^{2}=d d e f g \\
& \sigma_{2}=a b c e f g \\
& \sigma_{2} \mid \boldsymbol{s}^{1}=a b c \\
& \sigma_{2} \mid \mathbf{s}^{2}=e f g
\end{aligned}
$$

(c) $\log$ traces

Figure 6.2: Example of decomposition with transition boundary.

$\log$ trace fits the overall net if and only if it fits all the subnets. The reason for that is that when a transition is shared among subnets, the label of the transition is used to synchronize the subnets that contain that transition on their boundaries, ensuring that the decisions on model ability to reproduce that label are done jointly. Consider the decomposition $D_{\mathbb{D}}=$ $\left\{S N^{S_{1}}, S N^{S_{2}}\right\}$ from the example of Figure 6.2, where $S N^{S_{1}}=\left(P N^{S_{1}}\right.$, [start], []) and $S N^{S_{2}}=$ $\left(P N^{S_{2}}\right.$, [], [end] $)$ are the systems nets derived from the SESEs $S_{1}$ and $S_{2}$. Consider the trace $\sigma_{1}=a b c d d e f g$ shown in Figure 6.2c. Such trace does not fit the overall net due to the double $d$. The projection of that trace on $S N^{S_{1}}$ and $S N^{S_{2}}$ results in $\sigma_{1} \uparrow_{T^{S_{1}}}=a b c d d$ and $\sigma_{1} \uparrow_{T S_{2}}=$ ddefg respectively (cf. Figure 6.2c). Note that, although $\sigma_{1} \uparrow_{T^{S_{2}}}$ fits $S N^{S_{2}}$ (on $S N^{S_{2}}$, the preset of $d$ is empty hence it can fire more than once), $\left.\sigma_{1}\right|_{T^{S_{1}}}$ does not fit $S N^{S_{1}}$. Hence, the trace $\sigma_{1}$ that does not fit the overall net, does not fit all the subnets (at least there is one that is not fitting). A similar situation happens with the trace $\sigma_{2}=$ abcefg (where no $d$ appears), i.e., trace $\sigma_{2}$ does not fit the overall net, hence $\sigma_{2} \uparrow_{T^{S_{1}}}$ does not fit $S N^{S_{1}}$ or $\sigma_{2} \uparrow_{T^{S_{2}}}$ does not fit $S N^{S_{2}}$. In the latter example, actually both do not fit.

On the other hand, the case of place boundary is different. When the boundary (entry or exit) is a place, it is shared among two or more subnets. However, the arcs connected to the place (the ones in charge of producing and consuming tokens) are split among the subnets. This makes the place unable to synchronize, and therefore, it is impossible to analyze the different subnets in isolation. The example in Fig. 6.3 reflects this situation. The original net is decomposed into two subnets, $D_{\mathbb{D}}=\left\{S N^{S_{1}}, S N^{S_{2}}\right\}$, corresponding with the SESEs $S_{1}$ and $S_{2}$, and being $p$ the boundary place shared by both subnets. It can be seen that the arcs that produce tokens in $p$ and the ones that consume tokens from $p$ are distributed into different 


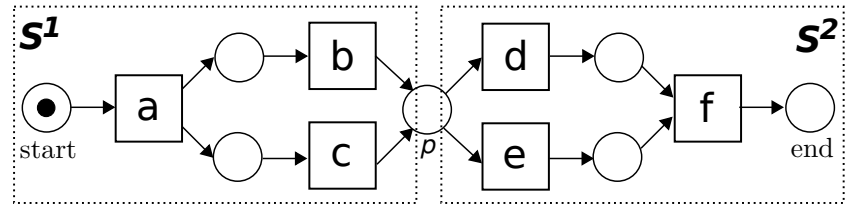

(a) original model

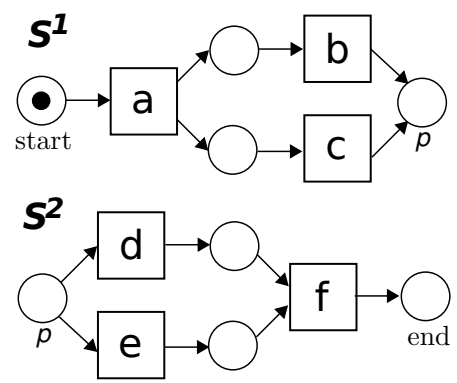

(b) decomposition

$$
\begin{aligned}
& \sigma_{1}=a b c d e f \\
& \sigma_{1} \mid s^{1}=a b c \\
& \sigma_{1} \mid s^{2}=d e f \\
& \sigma_{2}=a b d e c f \\
& \sigma_{2} \mid s^{1}=a b c \\
& \sigma_{2} \mid s^{2}=d e f
\end{aligned}
$$

(c) $\log$ traces

Figure 6.3: Example of decomposition with place boundary.

subnets. Consider now the $\log$ traces $\sigma_{1}=$ abcdef and $\sigma_{2}=$ abdecf of Fig. 6.3. While $\sigma_{1}$ fits the overall net, $\sigma_{2}$ does not. However, the projections of both traces on $T^{S_{1}}$ and $T^{S_{2}}$ are the same (cf. Fig. 6.3). This problem materializes when we analyze the subnets. Firstly, given that any arc that produces tokens in $p$ is contained in $P N^{S_{1}}$, we need to consider an initial marking for $S N^{S_{2}}$ different than [] (otherwise, the subnet would be deadlocked initially). If we consider the initial marking $[p], \sigma_{1} \Gamma_{T^{S_{2}}}$ does not fits $S N^{S_{2}}$. Therefore the fitness correctness is not preserved, i.e., a trace that fits the overall net like $\sigma_{1}$ must fit all the subnets. On the other hand, if we consider the initial marking with two (or more) tokens on $p$ (i.e., $\left[p^{2}\right]$ ), $\sigma_{2} \uparrow_{T^{S_{2}}}$ fits $S N^{S_{2}}$ (similarly, $\left.\sigma_{2}\right|_{T^{S_{1}}}$ fits $S N^{S_{1}}$ ). However $\sigma_{2}$ is a non-fitting trace of the overall net, and consequently, it must not fit all the subnets. Therefore, when the decomposition contains place boundaries, the preservation of the fitness correctness is not guaranteed.

In [1] the definition of decomposition is revisited to propose the so called valid decomposition, i.e., a decomposition that only shares transitions (but not places nor arcs).

Definition 6.8 (Valid Decomposition [1]). Let $S N=\left(W N, M_{I}, M_{F}\right)$ be a system net where $W N=(P, T, F$, start, end $) . D=\left\{S N^{1}, S N^{2}, \ldots S N^{n}\right\}$ is a valid decomposition of $S N$ if and only if:

- $T=\bigcup_{1 \leq i \leq n} T^{i}$,

- $P=\bigcup_{1 \leq i \leq n} P^{i}$ where $P^{i} \cap P^{j}=\emptyset$ for $1 \leq i<j \leq n$, 
- $F=\bigcup_{1 \leq i \leq n} F^{i}$ where $F^{i} \cap F^{j}=\emptyset$ for $1 \leq i<j \leq n$.

In [1, Theorem 2] it is proven that all valid decomposition preserves the fitting correctness, i.e., a log is fitting a system net if and only if fits all the subnets.

As has been illustrated in the previous examples, a decomposition based directly on SESEs is not necessarily a valid decomposition, i.e., boundary places may be shared among subnets. However, in the remainder of this section an approach to transform a SESE decomposition into a valid decomposition is presented, which tries to preserve the underlying semantics behind the SESE decomposition. This technique is called bridging, and consists of: (1) transforming each place boundary found into a transition boundary (i.e., boundary place is removed) and (2) creating explicit subnets (called bridges) for each boundary place. The bridges contain all the transitions connected with the boundary place, and they are in charge of keeping the place synchronized among subnets. In addition, the boundary places together with the arcs connected to them are removed from the original subnets. Formally:

Definition 6.9 (Bridging a SESE decomposition). Let $\mathbb{D}=\left\{S_{1}, \ldots S_{n}\right\}$ be SESE decomposition of the workflow net $W N=(P, T, F$, start, end $)$. Let $I_{\mathbb{D}}=\left\{i_{1}, \ldots, i_{n}\right\}$ and $O_{\mathbb{D}}=$ $\left\{o_{1}, \ldots, o_{n}\right\}$ be the set of all entry and exit nodes of the SESEs in $\mathbb{D}$. $B=\left\{p_{1}, \ldots, p_{k}\right\}=$ $\left(\left(I_{\mathbb{P}} \cup O_{\mathbb{P}}\right) \cap P\right) \backslash\{$ start, end $\}=\left(I_{\mathbb{P}} \cap O_{\mathbb{P}}\right) \cap P$ is the set of boundary places, i.e., entry and exit nodes of the SESEs that are places but not the source or sink place of the workflow net $W N$. The decomposition after applying bridging $\mathbb{D}^{\prime}=\left\{S_{1}^{\prime}, \ldots S_{n}^{\prime}, B_{1} \ldots B_{k}\right\}$ of $\mathbb{D}$ is constructed as follows:

- For all $1 \leq i \leq n: S_{i}^{\prime}=\left\{(x, y) \in S_{i} \mid\{x, y\} \cap B=\emptyset\right\}$ (boundary places are removed from the SESEs).

- For $1 \leq j \leq k: B_{j}=\left\{(x, y) \in A \mid p_{j} \in\{x, y\}\right\}$ (bridges are added). $\mathbb{D}^{\prime}$.

$D_{\mathbb{D}^{\prime}}=\left\{S N^{S_{1}^{\prime}}, \ldots S N^{S_{n}^{\prime}}, S N^{B_{1}} \ldots S N^{B_{k}}\right\}$ represents the decomposition constructed from

Figure 6.4 illustrates the effects of the bridging on the example previously shown in Fig. 6.3. In this case, the boundary place $p$ (and its arcs) are removed from $S_{1}$ and $S_{2}$, and a bridge $B_{1}$ is created. Note that now, the transitions connected to $p$ (i.e., $b, c, d$ and $e$ ) are shared (instead of $p$ ), keeping the synchronization among components, and making $D_{\mathbb{D}^{\prime}}$ a valid decomposition.

Proposition 6.10 shows that the decomposition derived from applying SESE decomposition and then bridging results in a valid decomposition, according to Def. 6.8.

Proposition 6.10 (Bridging results in valid decomposition). Let $\mathbb{D}^{\prime}=\left\{S_{1}^{\prime}, \ldots S_{n}^{\prime}, B_{1} \ldots B_{k}\right\}$ be obtained from a SESE decomposition after applying bridging. The decomposition $D_{\mathbb{D}^{\prime}}=$ $\left\{S N^{S_{1}^{\prime}}, \ldots S N^{S_{n}^{\prime}}, S N^{B_{1}} \ldots S N^{B_{k}}\right\}$ is a valid decomposition according to Def. 6.8. 


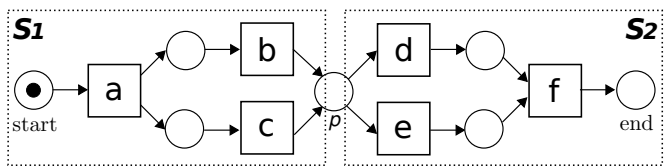

(a) original model

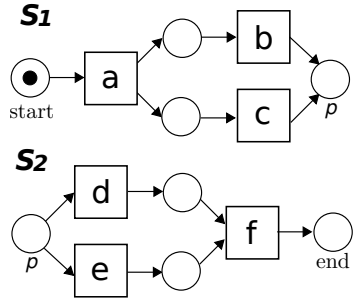

(b) decomposition

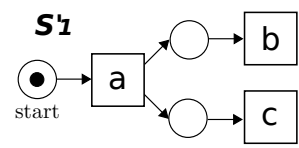

B1

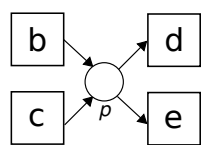

S

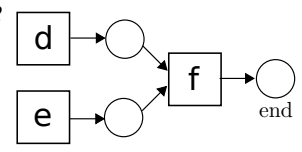

(c) decomposition and bridging

Figure 6.4: Example of decomposition with bridging.

Proof. By construction, a SESE decomposition only shares transitions and places. After applying the bridging, all the shared places are removed, creating explicit components with only one instance of these places.

Moreover, given that the bridging produces a valid decomposition, it also preserves the property that a trace in the log fits the overall process model if and only if each subtrace fits the corresponding process fragment. Hence, fitness checking can be decomposed using SESEs and bridges.

Proposition 6.11 (SESE-based Fitness Checking can be decomposed). Let L be a log and $S N=\left(W N, M_{I}, M_{F}\right)$ be a system net where $W N$ is a workflow net. Let $D_{\mathbb{D}^{\prime}}=$ $\left\{S N^{1}, S N^{2}, \ldots S N^{n}\right\}$ be a valid decomposition resulting of the application of the SESE decomposition and bridging over $W N$. Let $S N^{i}=\left(P N^{i}, M_{I}^{i}, M_{F}^{i}\right)$, where $P N^{i}=\left(P^{i}, T^{i}, A^{i}\right)$.

A trace $\sigma \in L$ fits $S N$ (i.e., $\left(W N, M_{I}\right)[\sigma\rangle\left(W N, M_{F}\right)$ ) if and only if it fits all the parts, i.e., for all $S N^{i} \in D_{\mathbb{D}^{\prime}},\left(P N^{i}, M_{I}^{i}\right)\left[\sigma \uparrow_{T^{i}}\right\rangle\left(P N^{i}, M_{F}^{i}\right)$.

Proof. Special case of the more general Theorem 2 in [1]. If the overall trace $\sigma$ fits $S N$, then each of the projected traces $\sigma \uparrow_{T^{i}}$ fits the corresponding subnet. If this is not the case, then at least there exist one projected trace $\sigma \Gamma_{T^{i}}$ that does not fit. But this is impossible because, by construction, each subnet is a relaxation of the behavior of the overall net. If the projected 
traces $\sigma\left\lceil_{T^{i}}\right.$ fit the corresponding subnets, then these traces can be stitched back into a trace $\sigma$ that fits $S N$.

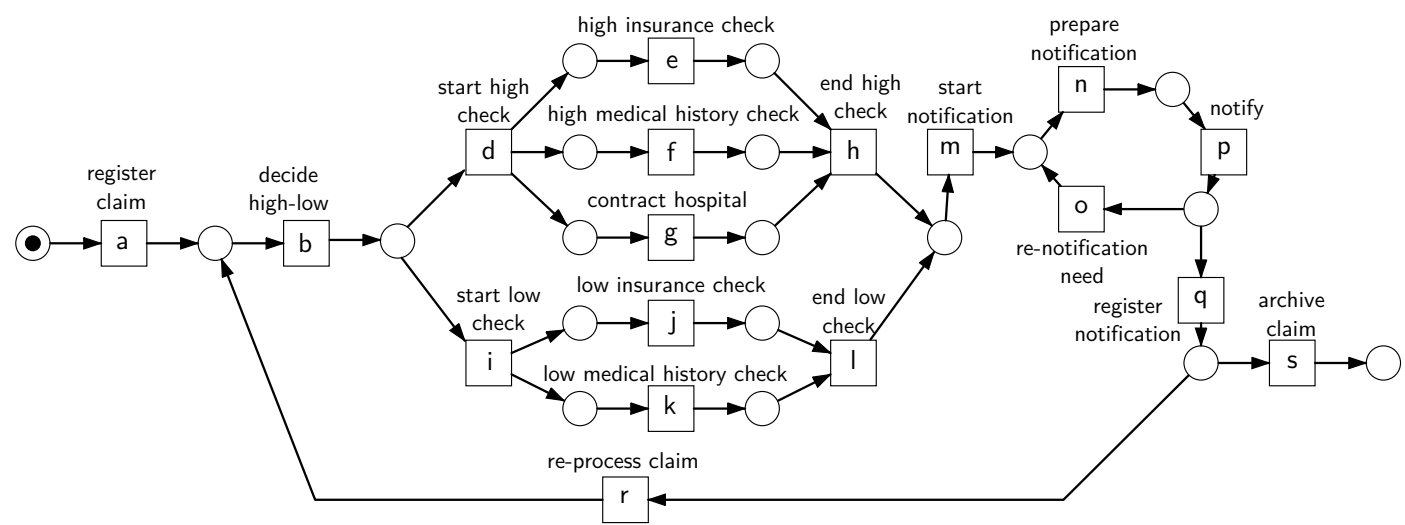

Figure 6.5: Running example: claims in a insurance company.

Let us consider the example of Figure 6.6 to illustrate the decomposition of the conformance checking proposed in this Chapter. The model in figure was inspired by a similar model presented in [24] and represents the possible situations to handle claims in a insurance company.

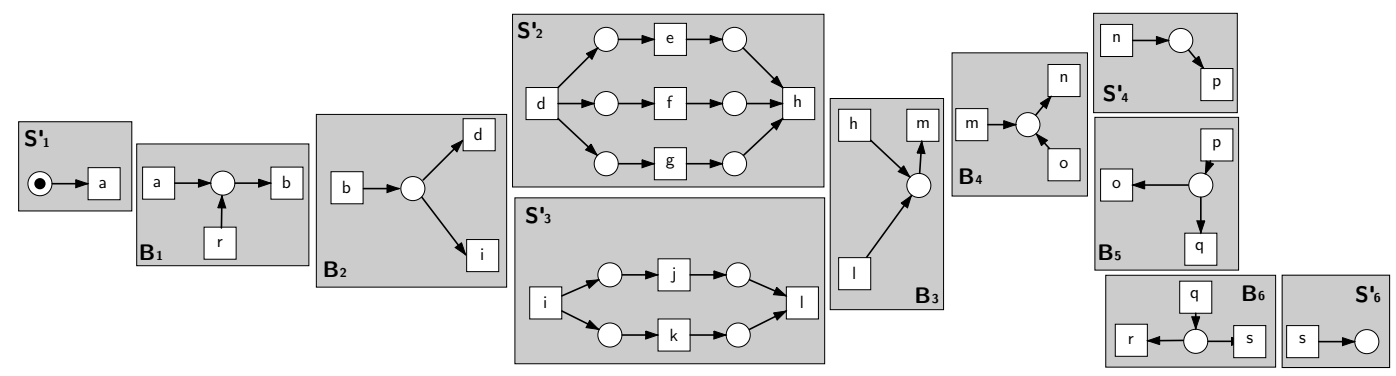

Figure 6.6: Components resulting from 15-decomposition and bridging for the running example of Figure 6.5.

Figure 6.6 shows the SESE decomposition using a size $k$ of 15 . Let us show how the fitness problems are now identified in a decomposed manner. For that, we will use the trace $\sigma=$ abijlmnpqnpqs. Given $\sigma$ and each one of the SESEs provided in Figure 6.6, the only ones that reveal fitness anomalies are $S_{3}^{\prime}, B_{4}$ and $B_{6}$ (for the other components we can find perfect alignments when projecting $\sigma$ to the activities of the component). The alignment for $S_{3}^{\prime}$ is: 


\begin{tabular}{|l|c|c|c|}
$i$ & $j$ & $\gg$ & $l$ \\
\hline$i$ & $j$ & $k$ & $l$
\end{tabular}

which reveals that the mandatory check of the medical history is missing in the log. Analogously, the alignment for $B_{4}$ is:

$$
\begin{array}{|c|c|c|c|}
m & n & \gg & n \\
\hline m & n & o & n
\end{array}
$$

that identifies the explicit need for notifying again the client, an action missing in the log but required by the model. Finally, the alignment for $B_{6}$ :

$$
\begin{array}{|c|c|c|}
q & q & s \\
\hline \gg & q & s
\end{array}
$$

reveals another fitness problem for trace $\sigma$ : the system has stored in the log an early registration of the notification which was not meant at that point in time, since later notifications were sent and according to the model, the registration is only expected to be done at the end of the case.

Using Prop. 6.11, we can infer that the fact that some components in the decomposition identify fitness problems implies that the whole model does not fit the log.

\subsubsection{Decomposition with invisible/duplicates}

So far, the approach presented in this chapter was assuming that all the Petri net transitions were associated with a unique single activity, i.e., a transition could be unambiguously identified by its label. In this section we lift this assumption in order to consider invisible and duplicate transitions. An invisible transition is a transition without activity associated, e.g., transitions included for routing purposes. Duplicate transitions are transitions with the same activity associated. For example, consider the net of Figure 6.7, which is a slight variation of the example in Figure 6.5. This model contains an invisible transition (represented in black) used to skip the execution of contract hospital, i.e., now contract hospital is optional. Moreover, the new model does not distinguish between high insurance check and low high insurance check, but the same action insurance check is modeled in two different parts of the model, i.e., is a duplicate activity. The Petri net definition is extended considering now a labeling function $l \in T \nrightarrow \mathcal{U}_{A}$ where $\mathcal{U}_{A}$ is some universe of activity labels. Additionally, if a transition $t \notin \operatorname{dom}(l)$, it is called invisible. $T_{v}(P N)=\operatorname{dom}(l)$ is the set of visible transitions in $P N . T_{v}^{u}(P N)=\left\{t \in T_{v}(P N) \mid \forall_{t^{\prime} \in T_{v}(P N)} l(t)=l\left(t^{\prime}\right) \Rightarrow t=t^{\prime}\right\}$ is the set of unique visible transitions in $P N$ (i.e., there are no other transitions having the same visible label)

As it has been illustrated previously in this chapter, when a net is decomposed, the labels of the transitions are used to synchronize and preserve the fitness properties. However, sharing invisible and duplicate transitions among subnets generates ambiguity invalidating this synchronization. Thus, the definition of valid decomposition presented in Definition 6.8 

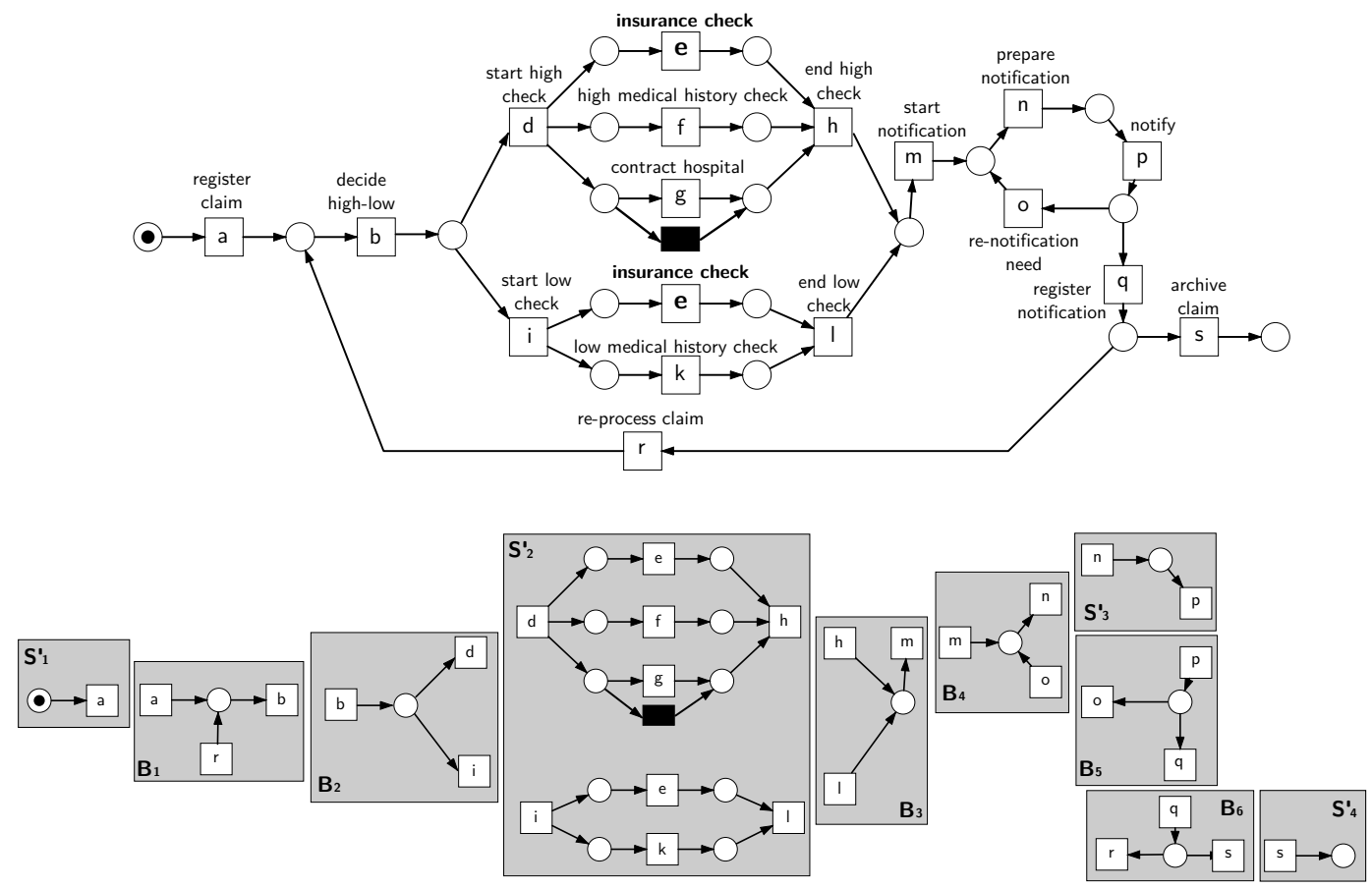

Figure 6.7: Variant of the running example of Figure 6.5 including invisible and duplicates (top), and its corresponding decomposition (bottom).

is refined to consider invisible and duplicates, i.e., only unique visible transitions can be shared among subnets.

Definition 6.12 (Valid Decomposition with Invisible and Duplicates[1]). Let $S N=\left(W N, M_{I}, M_{F}\right)$ be a system net where $W N=(P, T, F, l$, start, end $) . \quad D=\left\{S N^{1}, S N^{2}, \ldots S N^{n}\right\}$ is a valid decomposition of $S N$ if and only if:

- $S N^{i}=\left(P N^{i}, M_{I}^{i}, M_{F}^{i}\right)$ is a system net with $P N^{i}=\left(P^{i}, T^{i}, F^{i}, l^{i}\right)$ for all $1 \leq i \leq n$,

- $l^{i}=\left.l\right|_{T^{i}}$ for all $1 \leq i \leq n$,

- $P^{i} \cap P^{j}=\emptyset$ for $1 \leq i<j \leq n$,

- $T^{i} \cap T^{j} \subseteq T_{v}^{u}(S N)$ for $1 \leq i<j \leq n$, and

- $S N=\bigcup_{1 \leq i \leq n} S N^{i}$.

Let $S N=\left(W N, M_{I}, M_{F}\right)$ with $W N=(P, T, F, l$, start, end $)$ be a system net with valid decomposition $D=\left\{S N^{1}, S N^{2}, \ldots, S N^{n}\right\}$. We can observe the following properties: 
- each place appears in precisely one of the subnets, i.e., for any $p \in P: \mid\{1 \leq i \leq n \mid$ $\left.p \in P^{i}\right\} \mid=1$,

- each invisible transition appears in precisely one of the subnets, i.e., for any $t \in T$ $T_{v}(S N):\left|\left\{1 \leq i \leq n \mid t \in T^{i}\right\}\right|=1$,

- visible transitions that do not have a unique label (i.e., there are multiple transitions with the same label) appear in precisely one of the subnets, i.e., for any $t \in T_{v}(S N) \backslash$ $T_{v}^{u}(S N):\left|\left\{1 \leq i \leq n \mid t \in T^{i}\right\}\right|=1$,

- visible transitions having a unique label may appear in multiple subnets, i.e., for any $t \in T_{v}^{u}(S N):\left|\left\{1 \leq i \leq n \mid t \in T^{i}\right\}\right| \geq 1$, and

- each edge appears in precisely one of the subnets, i.e., for any $(x, y) \in F: \mid\{1 \leq i \leq n \mid$ $\left.(x, y) \in F^{i}\right\} \mid=1$.

In order to instantiate a decomposition complying with this new definition of valid decomposition, Algorithm 6.1 needs to be refined (cf. Algorithm 6.2).

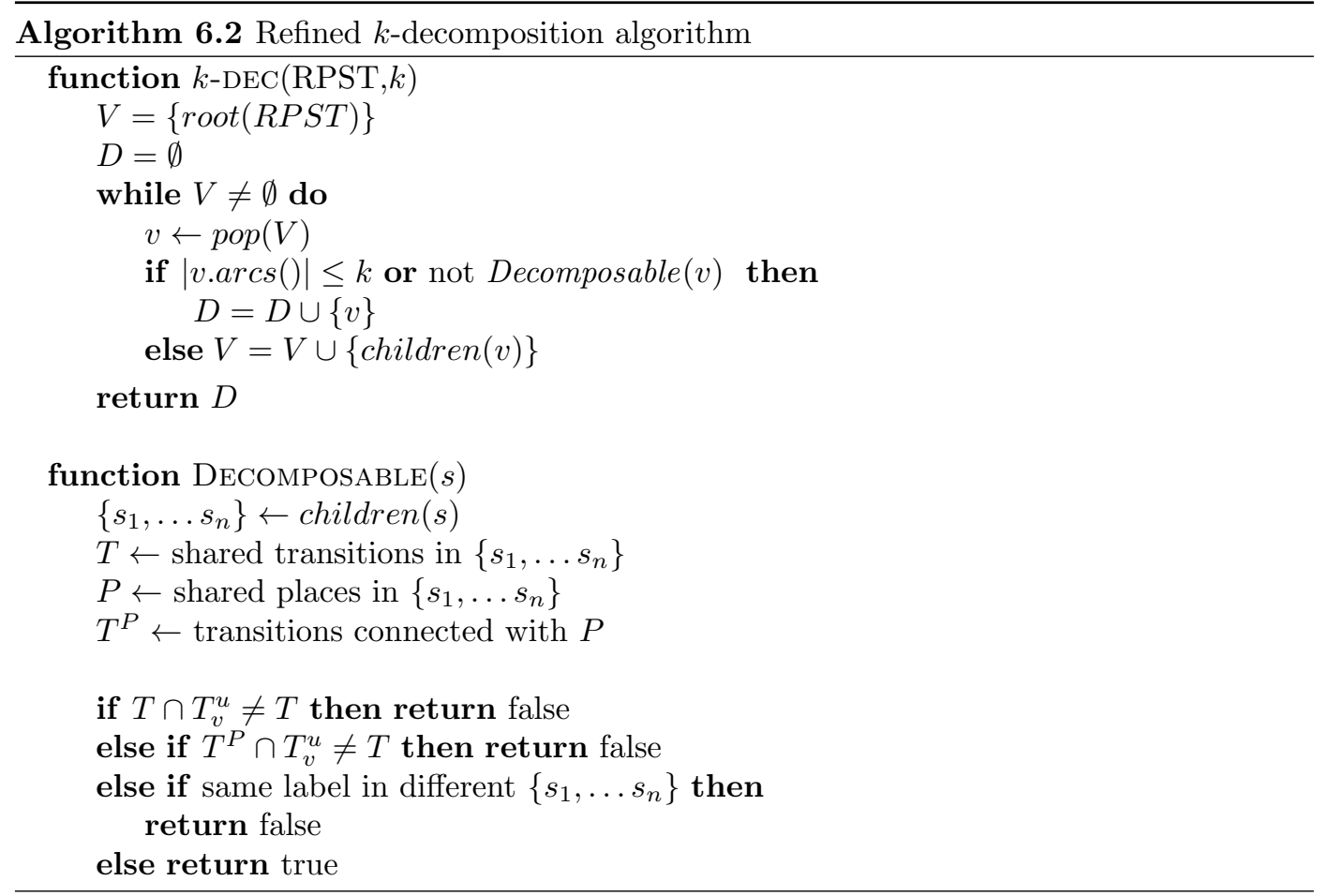


Algorithm 6.2 checks if considering the children of a SESE $s$ will violate the definition of valid decomposition in Definition 6.12. The three conditions need to be satisfied:

- transitions shared $(T)$ between any subset of SESEs $\left\{s_{1}, \ldots s_{n}\right\}$ must be unique visible transitions $\left(T_{v}^{u}\right)$.

- places shared $(P)$ between any subset of SESEs $\left\{s_{1}, \ldots s_{n}\right\}$ will be bridged according to Def. 6.9. Therefore, transitions connected with the places shared $(P)$ between any subset of $\left\{s_{1}, \ldots s_{n}\right\}$ must be unique visible transitions $\left(T_{v}^{u}\right)$, in order to avoid be duplicated boundary transitions after the bridging.

- Transitions with the same label must belong to the same $v_{i}$.

The main difference between the original $k$-decomposition algorithm presented previously and Algorithm 6.2 is that the latter checks if considering the children of SESE $v$ for the decomposition $D$ will violate the valid decomposition definition (Definition 6.12). Notice that by definition, if the children $\left\{s_{1}, \ldots s_{n}\right\}$ of $v$ violate the definition, considering further descendants of $v$ will also violate the definition. Therefore, when the algorithm checks that the SESE must not be decomposed, it includes it into the decomposition $D$. As a result, Algorithm 6.2 does not guarantee the $k$ property, i.e., some components may have more than $k$ arcs. For instance, consider the subnets resulting of a 15-decomposition and bridging shown in Figure 6.7. Unlike Figure 6.6, here when the algorithm tries to decompose the SESE $S_{2}$, it detects than this will result in splitting the duplicate $e$, and thus it must consider $S_{2}$, even if the number of $\operatorname{arcs}$ of $S_{2}$ is greater $15^{3}$. Notice that some worst case scenarios exist for Algorithm 6.2: consider the example of Figure 6.8. In this case, the presence of invisible transitions in the model boundaries makes it impossible for the algorithm decompose more that the root $S_{1}$, and therefore, the resulting decomposition will be the overall net. The effect of those cases can be alleviated by pre-processing the model and the log before applying the decomposed conformance.

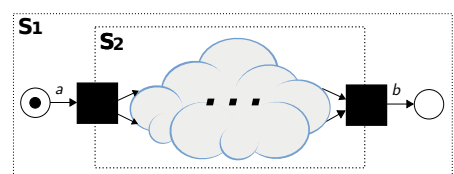

(a) workflow net

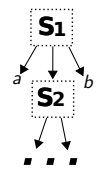

(b) RPST

Figure 6.8: Example of worst case scenario for the $k$-decomposition with invisible/duplicates.

\footnotetext{
${ }^{3}$ Notice that, the bridging may produce that a SESE loses its SESE structure, e.g., the entry and exit places of $S_{2}$ are removed when it becomes $S_{2}^{\prime}$ due to the bridges $B_{2}$ and $B_{3}$. In spite of this, the decomposition obtained still satisfies Definition 6.12.
} 


\subsection{Experimental Results}

In this section we provide experimental results demonstrating that decomposition approach proposed provides significant performance gains and improved diagnostics.

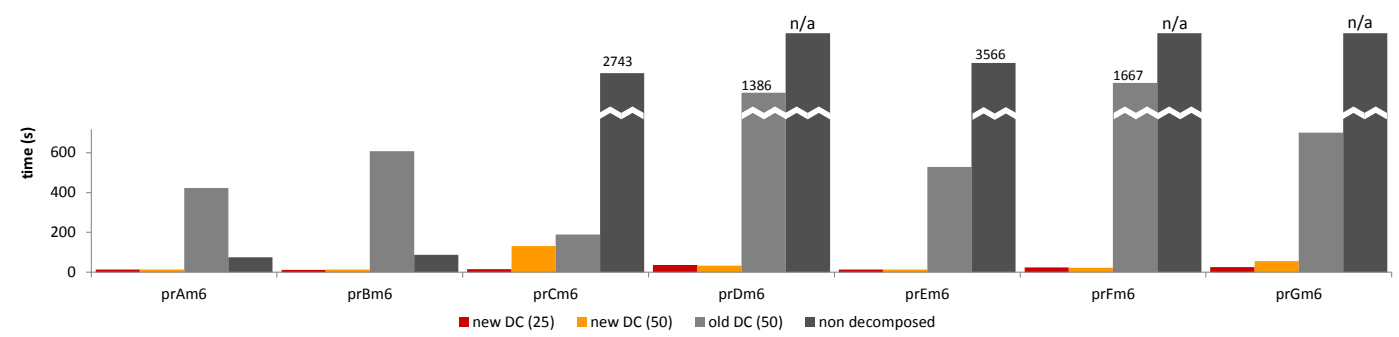

Figure 6.9: Comparison of computation time among different approaches: the new decomposed conformance checking technique (two variants: one which limits the maximum size of each component to $k=25$ and the other to $k=50$ ), the old decomposed conformance checking technique [65], and the approach without decomposition.

\section{Implementation}

The techniques presented in this chapter have been implemented within the ProM 6 tool $^{4}$, and have been included in the package Decomposed Conformance. The new implementation (unlike the one presented in $[66,65]$ ) is fully compliant with the Divide\&Conquer package a new initiative for a common framework among all decomposed process mining techniques within ProM, such as $[2,53,66,65]$. This modular framework is based on common objects and encourages and facilitates the reusability of plugins, both for discovery and conformance. Compared to the implementation in $[66,65]$ the architecture changed dramatically. For example, arrays of components are used, instead of the sequential creation and processing of the components. These changes in the implementation had a significant impact on the performance of the techniques. The Decomposed Conformance package in ProM supports all of the decomposition and filtering approaches and the advanced diagnostics described in this paper.

\section{Performance Improvements}

Figure 6.9 illustrates the performance improvement achieved by SESE-based decomposition and the new implementation in ProM. For this first analysis we use the bpm2013 benchmark $^{5}$. The benchmark contains large models with different levels of fitness (ranging from perfectly

\footnotetext{
${ }^{4}$ http: //www.promtools.org/prom6/nightly

${ }^{5}$ http://dx.doi.org/10.4121/uuid:44c32783-15d0-4dbd-af8a-78b97be3de49
} 
fitting as $\operatorname{pr} B m 6$, to models with fitness of 0.57 - like $\operatorname{pr} C m 6$ ), according to the fitness metric in [16]. Figure 6.9 compares four approaches using seven model-log combinations. The chart includes the results of the new Decomposed Conformance (DC), using a $k$ to decompose of 25 and 50 respectively (cf. Algorithm 6.1). In this experiment (and in the rest of section) we used a conformance based on alignments for checking the conformance of each component [16]. The comparison also includes the results of the previous implementation (with $k$ of 50) $[66,65]$, and the non-decomposed results of [16] (using the same algorithm and parameters as the decomposed approach).

The chart illustrates perfectly the vast difference, in computation time, between the approach presented and the non-decomposed alternative. The non-decomposed approach remains competitive for the less complex and highly fitting models (e.g., pr Am6 and $\operatorname{pr} B m 6$ ). Because of the component creation overhead the non-decomposed approach may even be faster for simple and well-fitting models as noted in [65]. For example, for $\operatorname{pr} A m 6$ and $\operatorname{pr} B m 6$ the non-decomposed approach is faster than the previous implementation presented in [65]. This is no longer the case for the new decomposed implementation which is outperforming the earlier approaches. In those cases where the complexity and fitness is an issue, the difference could reach two orders of magnitude (e.g., from 15 to 3566 seconds in prEm6). More importantly, the approach proposed is able to tackle and provide conformance information

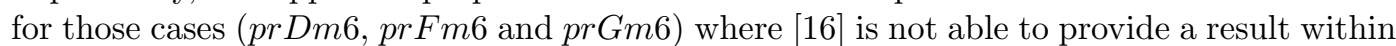
a period of 12 hours. Notice though, that the goal of both approaches is slightly different: while [16] aims for a global conformance, the decomposed approach aims for an optimal conformance of each component. However, a decomposed approach makes it possible to locate in a smaller vicinity where the conformance problems are, get a better understanding of the cause, and eventually be able to provide and bound conformance properties in a global manner [1]. The comparison also shows the significant speedup yield by the new implementation with respect to the one in [65], due to the new architecture based on arrays of components.

\section{Conformance Diagnosis}

One of the main contributions presented is a decomposed strategy to aid on the diagnosis of conformance problems in large systems, pinpointing which subprocesses are producing them. In order to illustrate this contribution we provide the fitness results per component for the running example and the benchmark bpm2013 (cf. Figure 6.10 and 6.11).

We use a circumference to graphically depict the fitness evaluation of a decomposition by means of a colored gradient for each component. All components of the decomposition are placed in different positions of the circumference. Let us use the running example of this chapter to illustrate the graphical visualization used.

For each component, a line from the center of the circumference indicates its fitness. If the line reaches the perimeter, the fitness is 1.0 (components $S_{1}^{\prime}, B_{1}, B_{2}, S_{2}^{\prime}, B_{3}, S_{4}^{\prime}, B_{5}, S_{6}^{\prime}$ ), while the line for components with fitness anomalies does not reach the perimeter. To show 


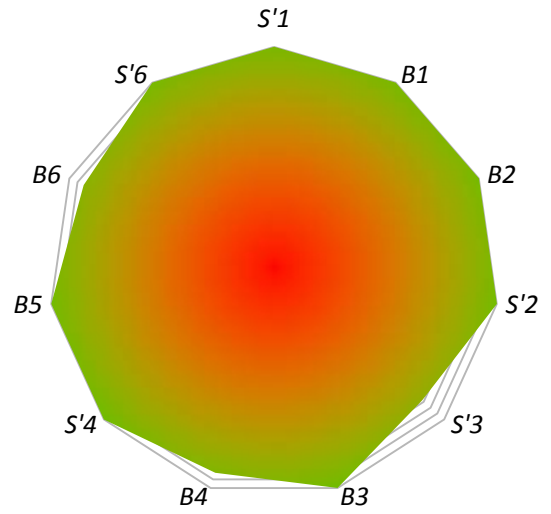

Figure 6.10: Fitness visualization for the running example.

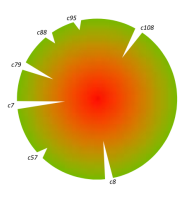

prAm6

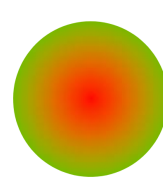

prBm6

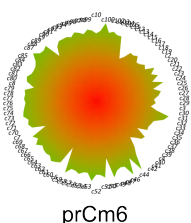

prCm6

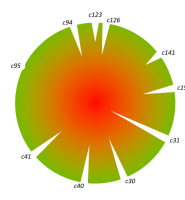

prDm6

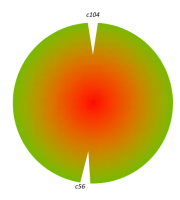

prEm6

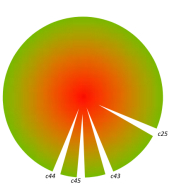

prFm6

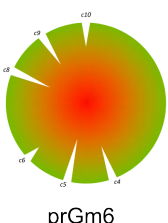

prGm6

Figure 6.11: Fitness results per components for benchmark bpm2013.

intuitively the fitness, a color gradient is included in the circumference: the fitness ranges from red/dark (fitness problems close to 0.0) down to green/light (perfect fitness of 1.0).

The fitness diagnosis of each one of the models of benchmark bpm2013 can be understood

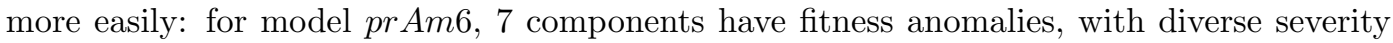
(7 dents on the circumference) . ${ }^{6}$ On the other hand, all components in $\operatorname{pr} B m 6$ are perfectly fitting. This contrasts with $\operatorname{prCm} 6$, where fitness problems are clearly spread over multiple components. The other of benchmark model-log combinations have fitness anomalies in just a few components. This supports the approach taken in this thesis. The diagnostics help to focus on the problematic parts while at the same time provide performance gains.

\section{Performance vs Comprehension}

The previous experiment included two different sizes for the decomposition: 25 and 50 . This second set of experiments is designed to determine the optimal decomposition size for both perspectives: performance impact and comprehension of the conformance anomalies

\footnotetext{
${ }^{6}$ When no fitness anomalies exist, we do not explicitly label components in the visualization.
} 
detected. The benchmark used, isbpm20137 , includes several models, and a set of logs with different properties for each model. In this experiment we have checked conformance for different values of $k$. Note that, when the $k$ is the total number of arcs of the model, the decomposed approach behaves as non-decomposed (i.e., there is only one component). Figure 6.12 reflects the computation time for two of the cases in the benchmark (pr1908-m34-l3noise and pr1151-m37-l3-noise), which summarizes the global tendency for all the models in the benchmark. The mark representing the minimum time is slightly differentiated.
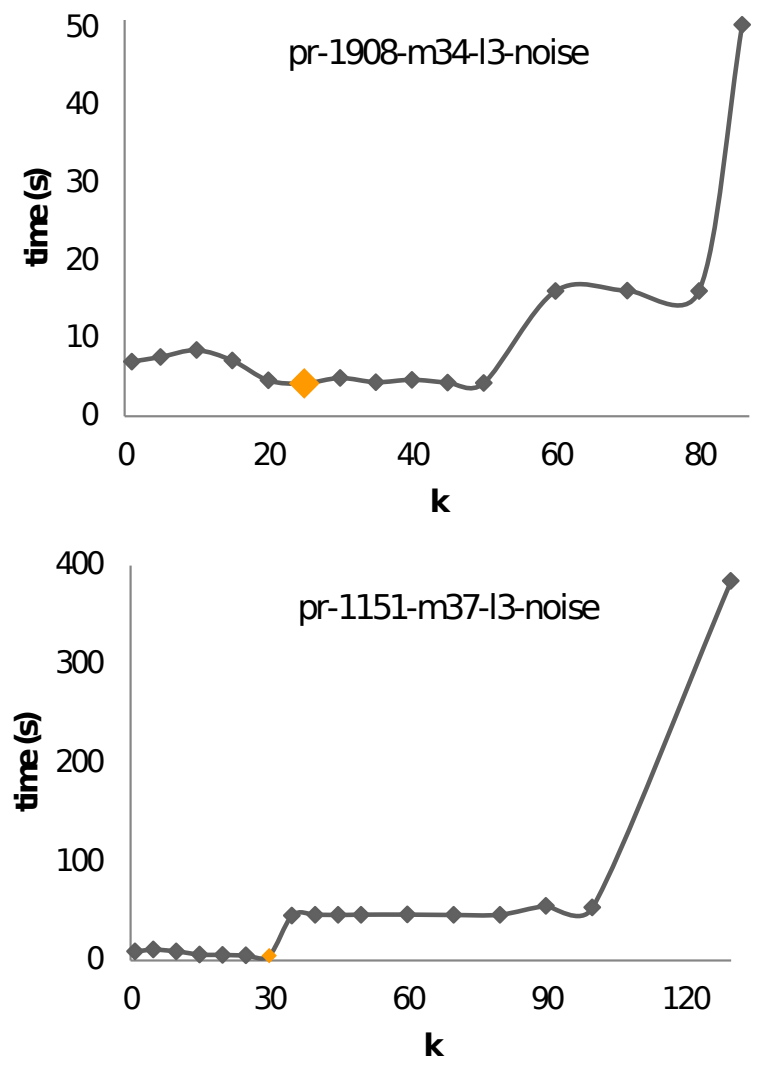

Figure 6.12: Comparison of computation time among different $k$ values.

The main conclusion one can reach from the experiments is that, from a computational time point of view, the smaller the better. This is perfectly reflected in Figure 6.12. For

\footnotetext{
${ }^{7}$ http://dx.doi.org/10.4121/uuid:b8c59ccb-6e14-4fab-976d-dd76707bcb8a
} 
small values of $k$ (e.g., $1 \ldots 20$ ), the results show a slight overhead because many components need to be created. However the effect of this overhead is negligible in most of the cases. On the other hand, when the $k$-decomposition starts to allow larger components, the computation time abruptly increases. This disruption on the computation time is produced by the hierarchical nature of the RPST, e.g., a decomposition $k$ instead of $k+1$ could lead the selection of $n$ subprocesses of low complexity instead of the one subprocess that includes all $n$. The results and insights based on these new experiments differ from [65], where-due to inefficiencies of the previous implementation- the overhead caused by the processing of components was significantly higher, making $k$ of 200 faster than 50 in some cases.

If components are excessively small (e.g., $1 \ldots 10$ ), the semantic information they provide is rather trivial or insufficient. From a diagnostic point of view, the subprocesses to be analyzed should be large enough to be able to make meaningful conclusions. Our empirical experiments show that, decomposition with $k$ between 20 and 40 could represent a good trade-off between computation time and diagnostic quality.

\section{Trace length and grouping}

A third set of experiments was conduced to study the effect of the trace lengths on the proposed approach. We aim to compare decomposed and non-decomposed conformance checking for different traces lengths. All logs and models of this experiments are included in the isbpm2013 benchmark. For each model used in this experiment, four logs were generated, each one with a different average length of the traces on it (e.g., pr1908-m18-l1 has an average trace length of 18, while pr1908-m41-l4 has average length of 41). Each one of these four logs has been generated from simulating the same model and using the same parameters (except the length of the traces), and all them are completely fitting. Additionally, we have created another four logs for each model, with the same characteristics, but containing noise (and hence being non-fitting). Figure 6.13 shows the results for two models: pr-1908 and pr-1151, being the results similar for the rest of models-logs in the benchmark. For each model, the chart contains the computation times of each alternative: decomposed (using $k$ of 25) with noisy logs and fitting logs, and the results for the same noisy and fitting logs using the original non-decomposed approach.

The first conclusion that arises from the experiments refers to the processes with noise - the most plausible assumption in a real world scenario. Figure 6.13 shows that, when the log has short traces, both decomposed and non-decomposed alignment checking perform good. However, once the length of the traces grows (or simply traces of large models or with lot of concurrency), it has a severe effect on the non-decomposed performance. This was to be expected, i.e., the more activities in a trace, the more difficult it is to compute the alignment. On the other hand, the decomposed approach performs both fast and with a near-to constant growth (and eventually constant at some point). This is justified by the effect of the decomposition on the computation time (as has been shown in Figure 6.9), but also due to the grouping (as explained below). 


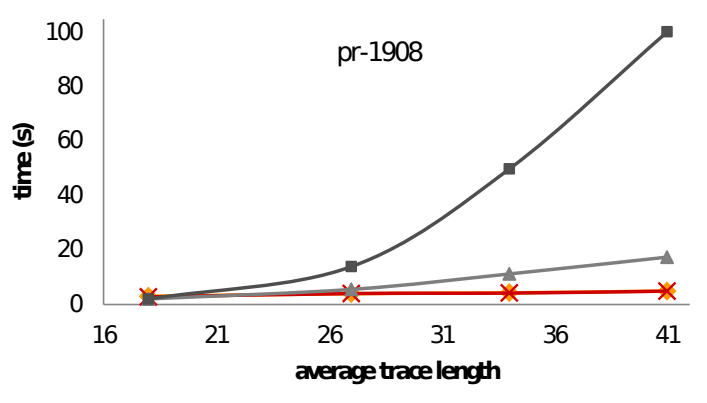

$\longrightarrow$ dec (25) fit $\rightarrow$ dec (25) noise $\rightarrow$ non_dec fit $\rightarrow-$ non_dec noise

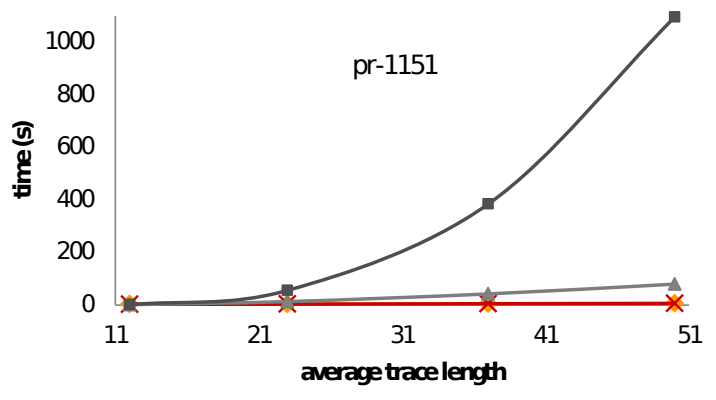

$\multimap$ dec (25) fit $\rightarrow$ dec (25) noise $\rightarrow$ non_dec fit $\rightarrow-$ non_dec noise

Figure 6.13: Comparison of computation time among different trace length.

The current implementation of the align-based conformance checking includes the grouping optimization: when the algorithm analyzes a trace, it first checks if it has already computed an alignment for an identical trace. In this is the case, it re-uses the previously computed alignment, thus reducing the time significantly. The effect of this optimization for the non-decomposed scenario depends on the case at hand, and it is strongly related with the size and the behavior of the model. However, in the decomposed scenario, the chances to encounter this situation increase: the smaller is the component (e.g., $k=25$ ), the fewer activities it contains, and therefore, the more likely it is to find a trace already seen before (once the original trace has been projected onto the component). The effects of the grouping are perfectly reflected by the fitting cases of Figure 6.13: the decomposed approach performs faster than the non-decomposed alternative even in a fitting scenario. This is remarkable because alignments can be created easily in this case. 


\subsection{Conclusions}

Checking conformance between an event log and a process model is a time demanding procedure, specially for those cases using conformance based on alignments. This chapter proposed the use of decomposed techniques to alleviate the time required to compute conformance, and to aid on the conformance diagnosis. The technique proposed is based on decomposing the model in single entry single exit components, denoting subprocesses within the main process with a simple interface with the rest of the model. The chapters provided formal proofs on the conformance analysis, and the experiments showed a significant reduction in computation time with respect to the monolithic approach. 



\section{Chapter 7}

\section{Topological and Multi-level Conformance Diagnosis}

\subsection{Introduction}

The main goal of decomposed conformance checking techniques is to alleviate the time required to analyze conformance, specially for complex alignment-based approaches. This is the case of the approach presented in Chapter 6 . However, in conformance analysis, computing the conformance metrics is as important as the diagnosis and understanding the cause of the conformance problems. Approaches that decompose processes into components provide a basic mechanism to diagnose conformance, assessing which components are problematic specially those techniques that decompose the process into meaningful components such as SESEs [67] or passages [2]. This chapters goes a step further in that direction, and provides additional mechanisms to diagnose conformance problems based on decomposed processes.

Comparing a process model with a map is a recurrent metaphor in process mining [6]. A map represents a city, like a models represents a process. There is not "a" map, but a set of maps for different purposes, for example street maps, traffic congestion maps, or real state maps. Maps can be decomposed, for example, into provinces, cities, or districts. Information in maps can be grouped, to indicate for example what areas concentrate most crimes, and they can allow to zoom-in and zoom-out to analyze this information at different levels. Figure 7.1 shows an example of real estate map, indicating the areas with more rent offers, being able to refine geographically this information with the zoom.

\footnotetext{
- The materials reported in this chapter are partially published in [67], [65] and [66]-
} 


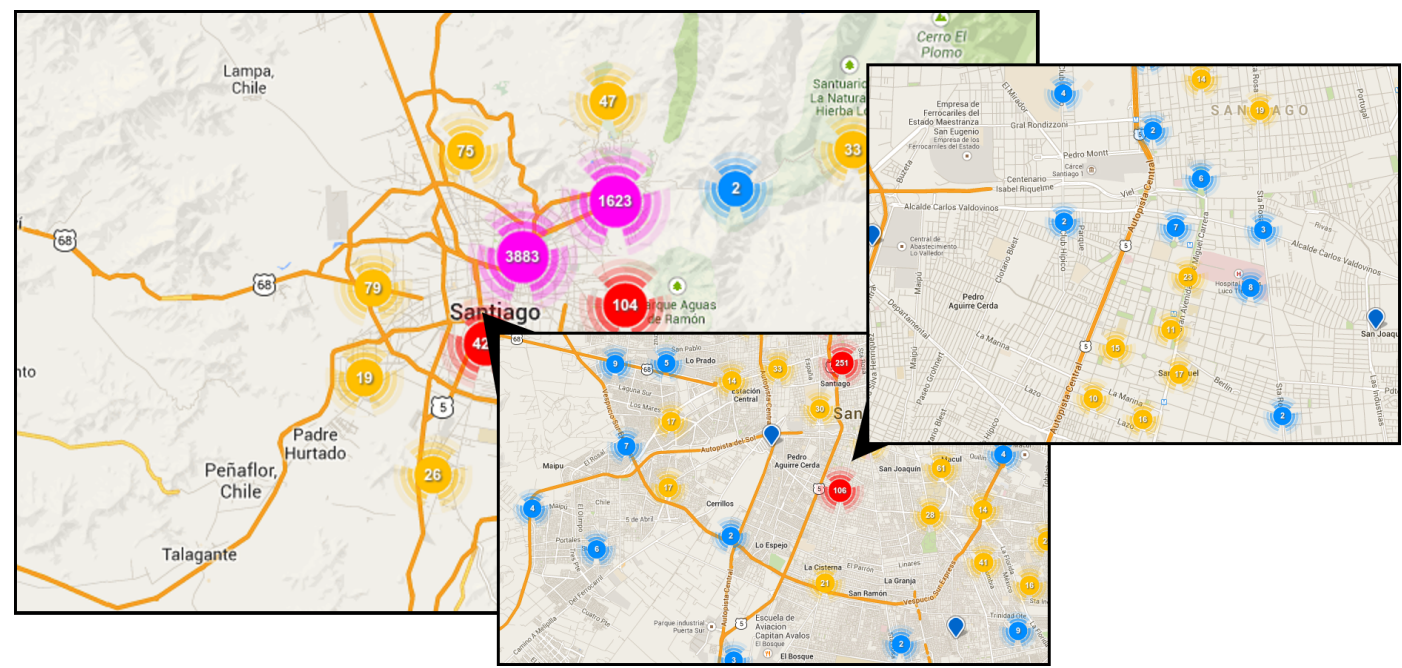

Figure 7.1: Interactive real estate map from www.portalinmobiliario.com, with grouping by proximity and zoom-in zoom-out functionality.

This chapter translates some of this map functionalities to decomposed conformance checking. In Section 7.2 we define a topological relationship between components, and use this relations to define close areas with conformance problems. Section 7.3 defines a hierarchy among components, opening the door to explore the conformance results at multiple levels. Moreover, the section proposes several refinements over the hierarchy to aid on the conformance diagnosis. Finally, Section 7.4 provides some experimental results, and Section 7.5 concludes the chapter.

\subsection{Topological Conformance Diagnosis}

A valid decomposition, presented in the previous chapter, is a collection of subnets that may be related to each other through the sharing of transitions, i.e., two subnets are related if they share a transition. The topology of a valid decomposition is an undirected graph where the vertices denote subnets and the edges denote the sharing of transitions.

Definition 7.1 (Topology of a Decomposition). Let $D=\left\{S N^{1}, S N^{2}, \ldots S N^{n}\right\}$ be a valid decomposition, where $S N^{i}=\left(P N^{i}, M_{I}^{i}, M_{F}^{i}\right)$ and $P N^{i}=\left(P^{i}, T^{i}, F^{i}\right)$. The topology of decomposition $D$ is defined as the undirected graph $T_{D}=(D, C)$ such that two components are connected if they share any transition, i.e., $C=\left\{\left\{S N^{i}, S N^{j}\right\} \mid 1 \leq i<j \leq n \wedge T^{i} \cap T^{j} \neq \emptyset\right\}$.

In the general definition of topology over a valid decomposition the relations remain undirected, i.e., two subnets sharing the same transition are connected by an undirected 
edge. However, in the specific case of a valid decomposition derived from SESEs, defined in Chapter 6, this definition can be extended to include the concept of direction: the transition being the exit of the SESE is considered the source of the edge, while the entry is the target. Bridges can have multiple entry and exit nodes, but again we can derive the direction connections among bridges and SESEs.

Definition 7.2 (Topology of a SESE Decomposition). Let $\mathbb{D}=\left\{S_{1}, \ldots S_{n}\right\}$ and $\mathbb{D}^{\prime}=$ $\left\{S_{1}^{\prime}, \ldots S_{n}^{\prime}, B_{1}, \ldots B_{k}\right\}$ be a SESE decomposition before and after applying bridging. Let $\left\{p_{1}, \ldots, p_{k}\right\}$ be the boundary places in $\mathbb{D}$. Let $D_{\mathbb{D}^{\prime}}=\left\{S N^{S_{1}^{\prime}}, \ldots S N^{S_{n}^{\prime}}, S N^{B_{1}} \ldots S N^{B_{k}}\right\}$ represent the decomposition constructed from $\mathbb{D}^{\prime}$. The topology of $D_{\mathbb{D}^{\prime}}$ is defined as the directed graph $T_{D_{\mathbb{D}^{\prime}}}=\left(D_{\mathbb{D}^{\prime}}, C\right)$ such that $C=\left\{\left(S N^{S_{i}^{\prime}}, S N^{S_{j}^{\prime}}\right) \mid 1 \leq i, j \leq n \wedge(y, x) \in S_{i} \wedge\right.$ $\left.(x, z) \in S_{j}\right\} \cup\left\{\left(S N^{S_{i}^{\prime}}, S N^{B_{j}}\right) \mid 1 \leq i \leq n \wedge 1 \leq j \leq k \wedge\left(y, p_{j}\right) \in S_{i}\right\} \cup\left\{\left(S N^{B_{j}}, S N^{S_{i}^{\prime}}\right) \mid 1 \leq\right.$ $\left.i \leq n \wedge 1 \leq j \leq k \wedge\left(p_{j}, y\right) \in S_{i}\right\}$.

Note that the topological graph has as vertices the nets in $\mathbb{D}^{\prime}$, but some arcs of this graph (those regarding connection to bridges) are defined over the original SESE decomposition $\mathbb{D}$, e.g., $\left(y, p_{j}\right) \in S_{i}$ refers to an arc in the original SESE and is used to infer a directed connection from $S N^{S_{i}^{\prime}}$ to $S N^{B_{j}}$.

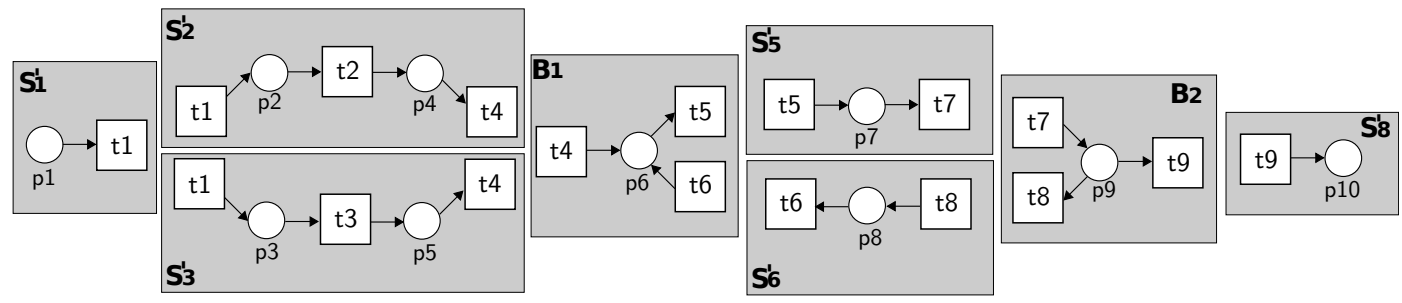

(a) decomposition and bridging

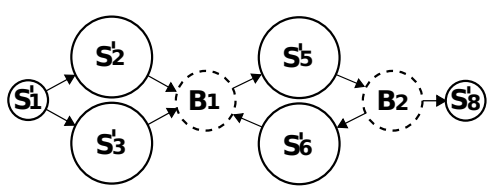

(b) topological graph

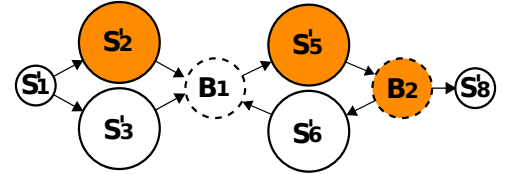

(c) topology enhanced with fitness for the trace t1 t3 t4 t5 t7 t7 t9

Figure 7.2: Example of valid decomposition and its topology

One of the features of the topology is to aid in the visualization of a valid decomposition. For example, let us consider the valid decomposition in Figure 7.2 (a slight modification of the model in Figure 6.1 in Chapter 6). The decomposition is the result of applying a 4-decomposition over the model (i.e., SESEs with at most 4 edges: $S_{1}^{\prime}, S_{2}^{\prime}, S_{3}^{\prime}, S_{5}^{\prime}, S_{6}^{\prime}, S_{8}^{\prime}$ ) and followed by the bridging (resulting in two bridges, $B_{1}$ and $B_{2}$, corresponding with the two 
boundary places $p_{6}$ and $\left.p_{9}\right)^{1}$. The corresponding topology is shown in same Figure 7.2b.

Besides simply showing the connections among subnets, the topology can be enhanced with other information about the components and their characteristics. For instance, bridges can be denoted by circles having dotted borders and SESEs can be denoted by circles having solid borders. Moreover, the size of the nodes in the graph is directly related with the size of the corresponding subnets, i.e., a subnet with many arcs is depicted using a larger circle compared to subnets with fewer arcs. Given the final goal of this thesis (i.e., conformance analysis), a particular interesting case is to enhance the topology with conformance information. For example, let us consider the trace $\sigma=t_{1} t_{3} t_{4} t_{5} t_{7} t_{7} t_{9}$. When we check fitness in the subnets of decomposition $D_{\mathbb{D}^{\prime}}=\left\{S N^{S_{1}^{\prime}}, \ldots S N^{S_{8}^{\prime}}, S N^{B_{1}}, S N^{B_{2}}\right\}$, we detect the following fitness anomalies: in $S N^{S_{2}^{\prime}}, t_{4}$ is fired without firing $t_{2}$; in $S N^{S_{5}^{\prime}}, t_{7}$ is executed twice, but this requires the firing of $t_{5}$ also twice; finally, in the bridge $S N^{B_{2}}, t_{7}$ is fired twice, but $t_{9}$ only once, leaving a token remaining in $p_{9}$. This information can be used to enhance the topology. As shown in Figure 7.2c the vertices have problems can be depicted in color (here $S_{2}^{\prime}, S_{5}^{\prime}$ and $B_{2}$ ).

Although the topology is an important aid for the process diagnosis by itself, it can also guide further analysis. For instance, the topological graph enhanced with conformance information can be used to identify maximal process fragments with fitness problems. This allows us to focus on the problematic parts of a model, discarding the parts without any fitness problems. Algorithm 7.1 describes a procedure that is based on detecting connected components $\left(C_{c}\right)$ on the graph induced by the non-fitting vertices. First, the topological graph $\left(T_{D}\right)$ is filtered, leaving only non-fitting vertices $(V)$. Then, the weakly connected components $\left(C_{c}\right)$ are detected: 1$)$ a random node $v_{1}$ is chosen, 2$)$ all nodes $\left\{v_{1}, \ldots v_{n}\right\}$ weakly connected (i.e., connected vertices without considering the direction of the edges) with $v_{1}$ are computed using a depth-fist search exploration and they constitute a new connected component, and finally 4) $\left\{v_{1}, \ldots v_{n}\right\}$ are removed from the graph and the exploration of connected components continues. For each connected component, we project the elements of the original net they refer to. Note that this algorithm prioritizes the connectivity among vertices resulting in weakly connected components. However, alternative versions of the algorithm yielding strongly connected components are possible. For instance, given the example of Figure 7.2c, two connected components are found as shown in Figure 7.3: one corresponding to $S N^{S_{2}^{\prime}}$ and the other to the union of $S N^{S_{5}^{\prime}}$ and $S N^{B_{2}}$.

The topological graph enhanced with conformance information can also be used to create one complete subnet that includes all non-fitting subnets of the decomposition, i.e., a connected set of vertices $V$ containing all the non-fitting vertices $V_{n f}$. Algorithm 7.2 illustrates the heuristic-based approach proposed, based on the greedy expansion of the largest non-fitting connected components, to compute the complete non-fitting subnet. Initially, $V$ contains the non-fitting vertices $V_{n f}$, and $G$ denotes the graph induced by $V$. The goal

\footnotetext{
${ }^{1}$ Note that the original trivial SESE $S_{4}$ that corresponds to the arc $(t 4, p 6)$ has disappeared once the bridging has been done, i.e., the arc is now in $B_{1}$. The same happens for the original trivial SESE $S_{7}$ corresponding to the arc $(p 9, t 9)$.
} 


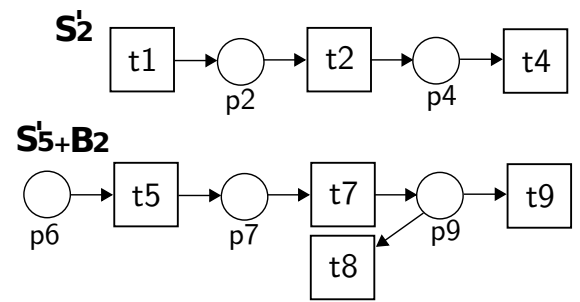

Figure 7.3: Examples of non-fitting weakly connected components.

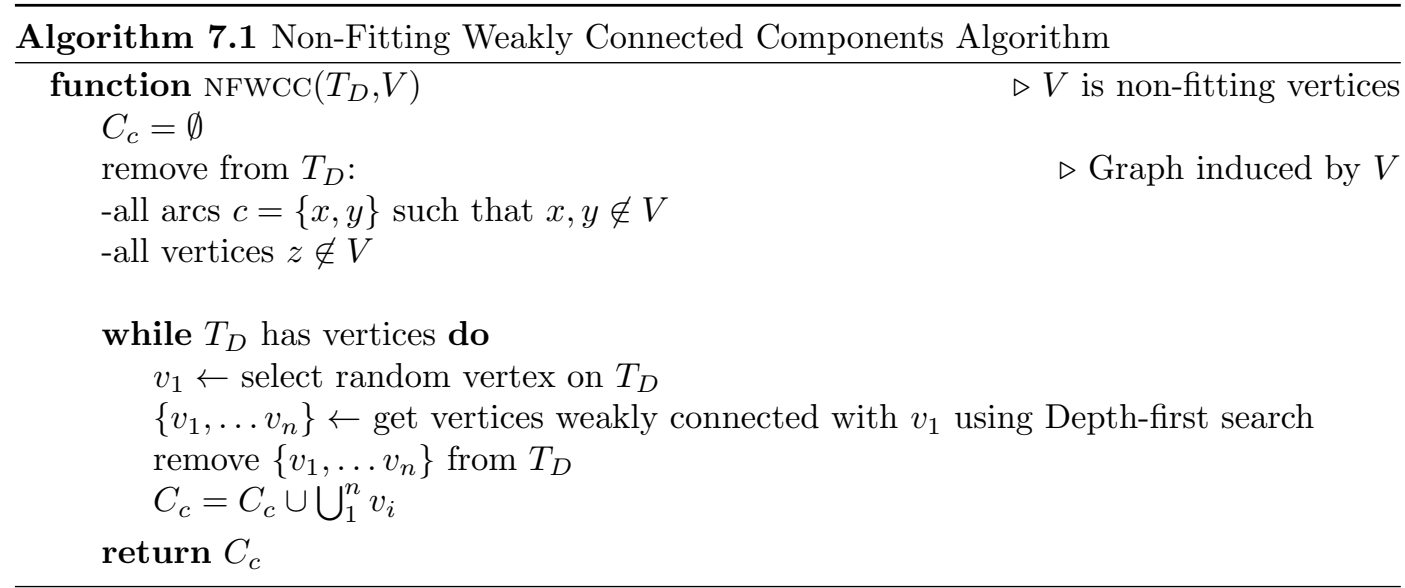

of the algorithm is to have all the vertices in $V$ connected, i.e. $G$ be connected. If this is not the case, the algorithm detects the two largest connected components $\left(c_{1}\right.$ and $\left.c_{2}\right)$ of $G$, and then computes the shortest path $\left(\left\{v_{1} \ldots v_{n}\right\}\right)$ between any vertex in $c_{1}$ and any vertex in $c_{2}$. Finally, $\left\{v_{1} \ldots v_{n}\right\}$ are included to $V$, and it is checked again if the induced graph $G$ is connected. Given the example of Figure 7.2c, the net resulting (shown in Figure 7.4) contains the union of the subnets $S N^{S_{2}^{\prime}}, S N^{S_{4}^{\prime}}, S N^{B_{1}}, S N^{S_{5}^{\prime}}$ and $S N^{B_{2}}$.

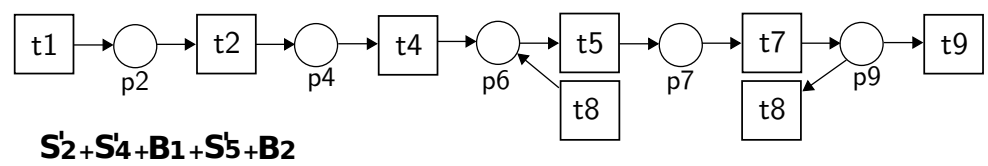

Figure 7.4: Example of a non-fitting subnet. 


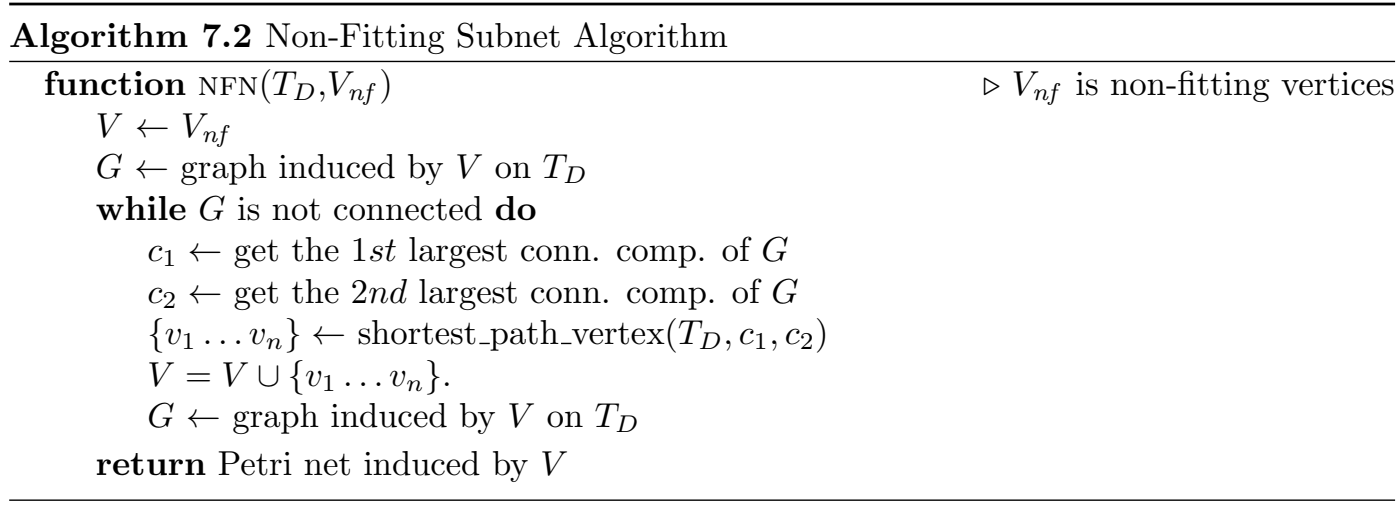

\subsection{Multi-level Conformance Diagnosis and its Applica- tions}

So far the analysis of the conformance was always performed using a complete decomposition of the model. However, for detailed process diagnosis it is important to also be able to do a more focused analysis. This section presents three approaches to achieve this: (1) stand-alone checking, (2) multi-level analysis, and (3) filtering.

\subsubsection{Stand-alone Checking}

First we consider the problem of analyzing a selected subprocess in isolation. Clearly, assumptions on the subprocess and its context must be defined in order to perform such an isolated conformance check. The conformance results obtained are strongly correlated with the assumptions considered, and hence the analysis of the model properties and domain knowledge becomes an essential part, e.g., whether a place has a bound on the number of tokens, or the number of activations of the subprocess within a trace.

Let us show an application of the stand-alone checking for the typical case of wellstructured process models, that can easily be modeled using the subclass of safe workflow nets [66]. Given a SESE $S$ obtained from a decomposition, one can apply the following steps to conduct a local diagnosis of $S$ :

1. Workflowing the SESE: In order to have a clear starting and ending point for the subprocess represented, re-define the net derived from $S$. In other words, given a SESE $S$, define the net derived from $S$ in terms of a workflow net, with an starting place (start) and a final place (end). By construction, a SESE has both an entry $(i)$ and an exit $(o)$ node. The start corresponds with $i$ if $i$ is a place. However, when $i$ is a transition, we define start to be an artificial place and we connect it with $i$. Similarly for end and $o$. 
2. Initial and Final Marking: Given the workflow-net from the previous step, determining a plausible initial marking becomes straightforward, i.e., due to the safeness assumption of safe workflow nets, we consider a single token in the start in order to enable the execution of the subprocess. Similarly for the final marking.

3. SESE activations: the number of potential activations of a SESE within a case must be determined. In case it is always one, the SESE is left as is. However, in case it can be executed more than once (e.g., the SESE is inside some loop in the model), the net in the previous step is short-circuited, using a silent transition between end and start. Finally, it can also happen that a SESE may be not executed in a trace. In this last case, a silent transition between start and end avoiding the SESE content will be used. Determining if a suprocess can be executed several times is a complex matter. In [66], it is proposed the use of Petri net structural theory (minimal T-invariants [88]) as a best effort strategy for estimating repetitive behavior.

\subsubsection{Multi-Level Analysis}

In this section we propose to combine the stand-alone checking presented above with the RPST to achieve a conformance analysis on a hierarchical manner. RPST nodes enriched with conformance information enable the analysis at different degrees of granularity and independence, similar to zooming in and out using online maps. Note that, by construction, the root of the RPST is the overall net. Therefore, any hierarchical analysis that involves the conformance checking of all the RPST nodes will require checking conformance on the original net (plus the checks of the rest of nodes), i.e., the computation time for a exhaustive hierarchical analysis will always be, by definition, greater than checking conformance on the overall net. For complex and time-consuming cases, this problem can be alleviated by limiting the size of the nodes to check or by using less expensive replay-based conformance techniques like $[80,30]$. The latter techniques use heuristics in order to deal with unfitting situations.

\subsubsection{Filtering}

The study presented in [66] suggest that there are three main differences between manual hierarchical decomposition and the one provided by the RPST-based decomposition: (1) analysts prefer to discard small components, (2) analysts prefer to not consider similar components, and (3) analysts prefer to have a hierarchy with a limited number of levels. Additionally, in this paper we point out a fourth difference: (4) analysts prefer to base hierarchies on other (non-control-flow) perspectives. In the remainder of this section we propose filtering techniques to allow for RPST-based decompositions closer to hierarchical decompositions preferred by analysts.

- Small components: Small components of the RPST can be removed by filtered using a minimal size threshold. 


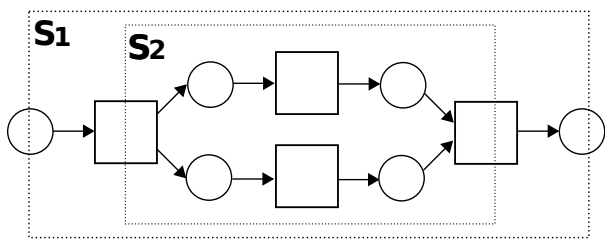

(a) similar size among SESEs

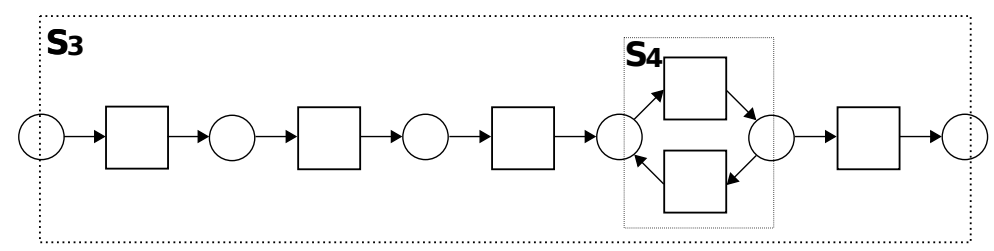

(b) high simplicity among SESEs

Figure 7.5: Example of cases with high similarity between nested SESEs.

- Similarity: In order to reduce the redundancy of components and the unnecessary growth of the hierarchy, a similarity metric between parent-child components is defined, together with a threshold that determines the similarity frontier that will determine when two components are considered essentially the same. The proposed metric for estimating the similarity between a node $S$ and its single child $S^{\prime}$ is based on two factors: size and simplicity. The size factor is related with the number of arcs of $S$ not included on $S^{\prime}$. The more arcs shared by both components, the more similar they are. For instance, considering the component $S_{1}$ of Figure 7.5a, all its arcs are included in $S_{2}$ except two, i.e., $S_{2}$ is in essence $S_{1}$. Therefore, a detailed conformance diagnosis over $S_{1}$ may be sufficient for understanding both subprocesses. The simplicity factor refers to the simplicity of part of the parent $S$ not included on the child $S^{\prime}$. When such part defines a simple behavior (e.g., the strictly sequential behavior of $S_{3}$ not included in $S_{4}$, in Figure 7.5b), the analysis and understanding of the parent may again be enough. On the other hand, when the behavior not included in $S^{\prime}$ contains complex controlflow constructs (e.g., mixtures of concurrency and choice) it may be more advisable to analyze both subprocesses. An example similarity metric is formalized as follows.

Definition 7.3 (Similarity Metric). Let $S_{P}=\left(V_{P}, F_{P}\right)$ be an RPST node, and let $S_{C}=\left(V_{C}, F_{C}\right)$ be its only child. Let size define the difference on size between them, i.e., size $=\left|F_{C}\right| /\left|F_{P}\right|$. Let $F_{O}=F_{P} \backslash F_{C}$ be the set of non-intersecting arcs. Let $F_{O}^{*}$ be the arcs in $F_{O}$ that have a source vertex with only one outgoing edge, and a target vertex with only one incoming edge, i.e., $F_{O}^{*}=\left\{(x, y) \in F_{O}\left|(x, v) \in F_{O}\right|=\right.$ $\left.1 \wedge\left|(w, y) \in F_{O}\right|=1\right\}$. Let simplicity define the simplicity of the non-intersecting arcs, i.e., simplicity $=\left|F_{O}^{*}\right| /\left|F_{O}\right|$. The similarity between $S_{P}$ and $S_{C}$ is the harmonic 
mean between size and simplicity:

$$
\text { similarity }=2 \cdot \frac{\text { size } \cdot \text { simplicity }}{\text { size }+ \text { simplicity }}
$$

Although the similarity evaluation is restricted to nodes with only one child, our experimental results show that the reduction achieved on the RPST may be significant (specially after applying a small nodes filtering).

- Multi-perspective filtering: The filtering presented until now is based on only structural net properties, not taking into account other perspectives (e.g., data, costs, roles, departments). However, they may be situations where we would like to focus the analysis only on those subprocesses satisfying certain domain conditions, e.g., an analyst may want to focus on the subprocesses involving tasks executed in a particular department. Therefore, we need to support filtering based on user-requirements and focus the analysis on the subprocesses involving activities relevant from the selected viewpoint. Such filtering is not limited to activities and may involve other perspectives (e.g., resources, actors, or costs), determining the activities they are connected with, and using them for filtering.

\subsection{Experimental Results}

The set of experiments of this section is designed to illustrate the effects of some of the techniques proposed for process diagnosis. In particular, the Non-fitting Subnet Algorithm (cf. Algorithm 7.2), and the techniques of filtering the RPST based on small components and similarity (cf. Section 7.3.3). Table 7.1 shows the application of the NFN algorithm over the benchmark bpm20132 , with components of size at most 50. For each model (containing $P$ places and $T$ transitions) the table provided the size of the minimal net containing all the non-fitting components, i.e., the number of places and transitions $(|P|$ and $|T|)$, and the number of vertices $|V|$ used to create the net. The table illustrates the benefits of the proposed algorithm to detect and isolate the fitness mismatches. In case the fitness problems are spread all over the whole model, the resulting net is almost the original net (e.g., $\operatorname{prCm6}$ ). However, when the fitness problems are local, the net that encloses all problem spots may be orders of magnitude smaller than the original net, thus easing the diagnosis.

The second experiment performed illustrates the effects of the simplification techniques over the RPST. Figure 7.6 reflect the results for one of the models $(\operatorname{prCm} 6)$. The charts show the number of nodes of the original RPST, after filtering small components $(<10)$ and then merging by similarity $(>0.8)$. The number of nodes are distributed by levels of depth in the RPST tree, i.e., the distance with the root represented as the level 1. The chart clearly reflects the difference between the number of components on the original RPST and

\footnotetext{
${ }^{2}$ http://dx.doi.org/10.4121/uuid:44c32783-15d0-4dbd-af8a-78b97be3de49
} 


\begin{tabular}{cccccccc}
\hline \multicolumn{3}{c}{ Dataset } & & \multicolumn{3}{c}{ NFN } \\
\cline { 1 - 3 } \cline { 5 - 7 } prAm6 & $P$ & $T$ & & $|V|$ & $|P|$ & $|T|$ \\
\cline { 2 - 3 } \cline { 5 - 7 } prCm6 & 317 & 317 & & 114 & 15 & 14 \\
prDm6 & 529 & 429 & & 31 & 55 & 517 \\
prEm6 & 277 & 275 & & 31 & 29 & 40 \\
prFm6 & 362 & 299 & & 7 & 27 & 25 \\
prGm6 & 357 & 335 & & 5 & 34 & 29 \\
\hline
\end{tabular}

Table 7.1: Results of NFN algorithm.

the one after removing the small components, i.e., most of the RPST nodes are small. After removing small nodes the depth of the RPST only decreases two levels (from 14 to 12). On the other hand, when merging on similarity is applied over the filtered RPST, the number of nodes is not reduced so drastically, but the number of levels of the tree is (from 13 to 6 ), providing a hierarchical decomposition with less redundancy and more aligned with the human perception [66].
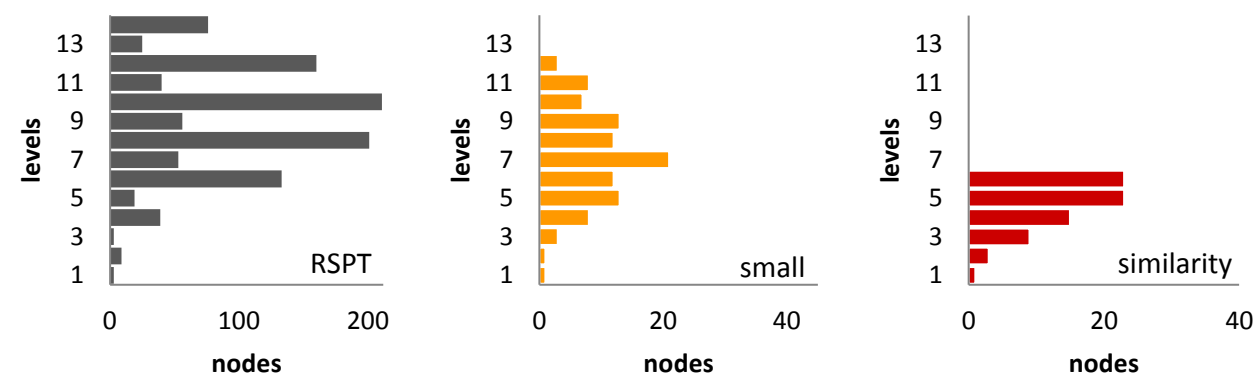

Figure 7.6: Results of filtering by small $(<10)$ and merging by similarity $(>0.8)$ over the

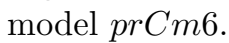

\subsection{Conclusions}

Decomposition techniques in conformance checking provide an efficient mechanism to identify and locate conformance anomalies. This chapter went a step further, using decomposition techniques to provide other mechanisms for the diagnosis of conformance problems. The chapter proposed a topological relation between components, used to identify closely connected components with conformance problems. Furthermore, the chapter defined a hierarchy of components, opening the door to a zoom-in zoom-out analysis of the conformance. 


\section{Chapter 8}

\section{Data-aware Decomposed Conformance Checking}

\subsection{Introduction}

Most of the work done in conformance checking in the literature, and the one presented in this thesis so far, focuses on the control-flow of the underlying process, i.e. the ordering of activities $[80,16]$. In a data-aware process model, each case, i.e. a process instance, is characterized by its case variables. Paths taken during the execution may be governed by guards and conditions defined over such variables. A process model specifies the set of variables and their possible values, guards, and write/read actions. Since existing conformance checking techniques typically completely abstract from data, resources, and time, many deviations remain undetected. Therefore, the event log may record executions of process instances that appear fully conforming, even when it is not the case. Rigorous analysis of the data perspective is needed to reveal such deviations.

Let us consider the process that is modeled as BPMN diagram in Figure 8.1. It models the handling of loans requests from customers. It is deliberately oversimplified to be able to explain the concepts more easily. The process starts with a credit request where the requestor provides some documents to demonstrate the capability of paying the loan back. These documents are verified and the interest amount is also computed. If the verification step is negative, a negative decision is made, the requestor is informed and, finally, the negative outcome of the request is stored in the system. If verification is positive, an assessment is made to take a final decision. Independently of the assessment's decision, the requestor is

- The materials reported in this chapter are partially published in [55] - 


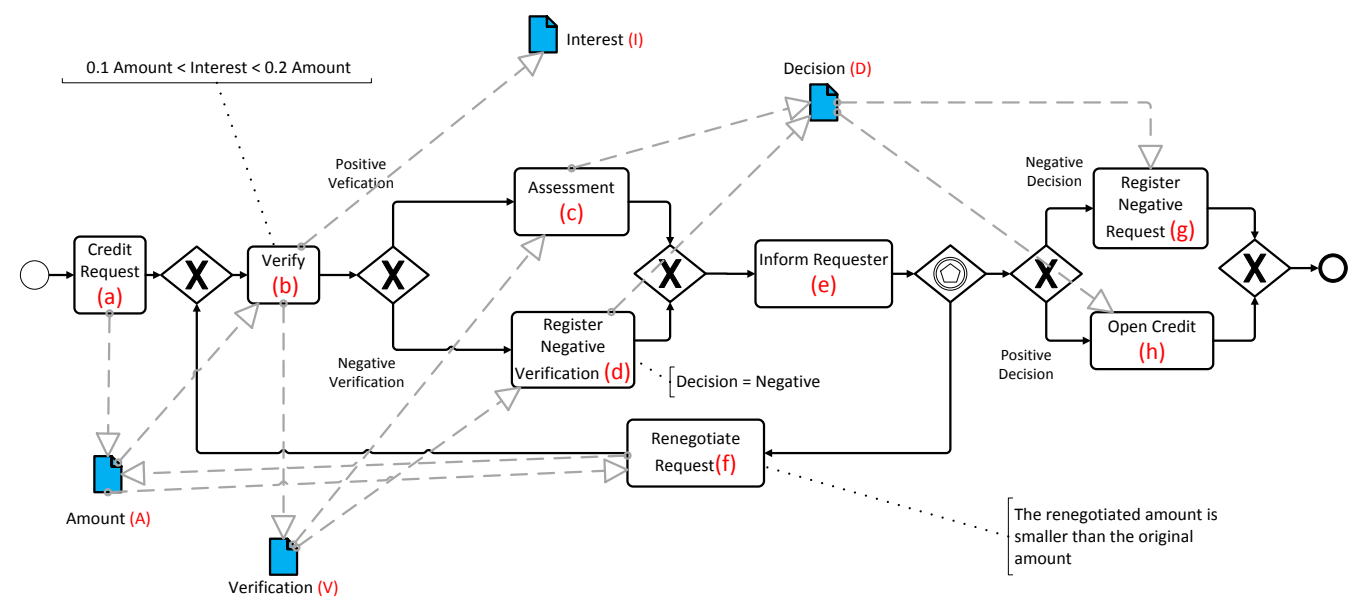

Figure 8.1: Example of a (simplified) process to request loans. The dotted arcs going from a transition to a variable denote write operations; the arcs towards a transition denote read operations, i.e. the transition requires accessing the current variables' value. Each transition is abbreviated into a lower-case letter (e.g. $a$ ) and each variable is represented as a upper-case letter (e.g. A). The abbreviations are shown in brackets after the name of the transitions or variable names.

informed. Moreover, even if the verification is negative, the requestor can renegotiate the loan (e.g. to have lower interests) by providing further documents or by asking for a smaller amount. In this case, the verification-assessment part is repeated. If both the decision and verification are positive and the requestor is not willing to renegotiate, the credit is opened. Let us consider the following trace: ${ }^{1}$

$$
\begin{gathered}
\sigma_{e x}=\langle(\mathbf{a}, \emptyset,\{(A, 4000)\}),(\mathbf{b},\{(A, 4000)\},\{(I, 450),(V, \text { false })\}),(\mathbf{c},\{(V, \text { false })\}, \\
\{(D, \text { true })\}),(\mathbf{e}, \emptyset, \emptyset),(\mathbf{f},\{(A, 4000)\},\{(A, 5000)\}),(\mathbf{b},\{(A, 5000)\},\{(I, 450), \\
(V, \text { false })\}),(\mathbf{d},\{(V, \text { false })\},\{(D, \text { false })\}),(\mathbf{e}, \emptyset, \emptyset),(\mathbf{h},\{(D, \text { true })\}, \emptyset)\rangle
\end{gathered}
$$

Seen from a control-flow perspective only (i.e. only considering the activities' ordering), the trace seems to be fully conforming. Nonetheless, a number of deviations can be noticed if the data perspective is considered. First of all, if activity $c$ is executed, previously activity $b$ could not have resulted in a negative verification, i.e. $V$ is set to false. Second, activity $f$

\footnotetext{
${ }^{1}$ Notation (act, $\left.r, w\right)$ is used to denote the occurrence of activity act that writes and reads variables according to functions $w$ and $r$, e.g., (b, $\{(A, 4000)\},\{(I, 450),(V$, false $)\})$ is an event corresponding to the occurrence of activity $\mathbf{b}$ while reading value 4000 for variable $A$ and writing values 450 and false to variables $I$ and $V$ respectively. $(\mathbf{e}, \emptyset, \emptyset)$ corresponds to the occurrence of activity $\mathbf{e}$ without reading/writing any variables.
} 
cannot write value 5000 to variable $A$, as this new value is larger than the previous value, i.e. 4000. Furthermore, if the decision and verification are both negative, i.e. both $V$ are $D$ are set to false, then $h$ cannot be executed at the end.

The identification of non-conforming traces clearly has value in itself. Nonetheless, organizations are often interested in explanations that can steer measures to improve the quality of the process. Alignments (cf. Chapter 5) aim to support more refined conformance checking. An alignment aligns a case in the event log with an execution path of the process model as good as possible. If the case deviates from the model, then it is not possible to perfectly align with the model and a best matching scenario is selected. Note that for the same deviation, multiple explanations can be given. For instance, the problem that $h$ was executed when it was not supposed to happen can be explained in two ways: (1) $h$ should not have occurred because $V$ and $D$ are both set to false ("control-flow is wrong") and (2) $V$ and $D$ should both have been set to true because $h$ occurs ("data-flow is wrong"). In order to decide for the most reasonable explanation, costs are assigned to deviations and we aim to find the explanation with the lowest cost. For instance, if assigning a wrong value to $V$ and $D$ is less severe than executing $h$ wrongly, the second explanation is preferred. The seminal work in [16] only considers alignments in the control-flow part, thus ignoring the data-perspective aspect of conformance.

As we detail in Section 8.2.3, finding an alignment of an event log and a data-aware process model is undecidable in the general case. However, to make the problem decidable, works $[54,57]$ put forward the limitation that guards need to be linear (in)equations. These works also show that, even with that limitation, the problem of finding an alignment of an event log can become intractable since the problem's complexity is exponential on the size of the model, i.e. the number of activities and data variables.

In this chapter, while keeping the limitations mentioned above, we aim to speed up the computation of alignments by using a divide-and-conquer approach. The data-aware process model is split into smaller partly overlapping model fragments. For each model fragment a sublog is created by projecting the initial event log onto the activities used in the fragment. Given the exponential nature of conformance checking, this may significantly reduce the computation time. If the decomposition is done properly, then any trace that fits into the overall model also fits all of the smaller model fragments and vice versa. The remainder of this chapter is organized as follows. Section 8.2 revisits the background definitions of conformance checking and process mining presented so far in this thesis to incorporate data information. In Section 8.3 we extend the definition of valid decomposition for data-aware models. Section 8.4 proposes a valid decomposition strategy based on SESEs. Experimental results, both artificial and real, are presented in Section 8.5. Finally, Section 8.6 concludes the chapter. 


\subsection{Data-aware Processes}

In the previous chapters, all the definitions and theory presented focus on the control-flow perspective of the processes. In this section we extend those concepts to incorporate the data perspective. In particular, this section presents Petri nets with data as data-aware process model notation, event logs with data, and the relation between models and logs.

\subsubsection{Petri nets with Data}

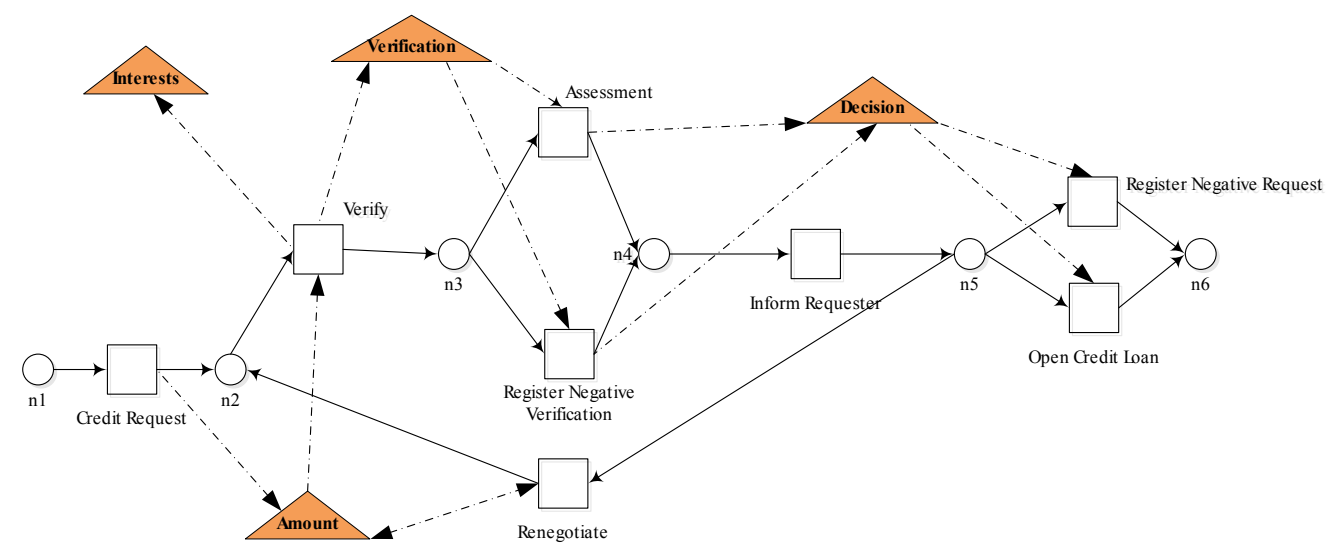

Figure 8.2: Pictorial representation of a Petri net with Data that models the process earlier described in terms of BPMN diagram (cf. Figure 8.1). Places, transitions and variables are represented as circles, rectangles and triangles, respectively. The dotted arcs going from a transition to a variable denote the writing operations; the reverse arcs denote the read operations, i.e. the transition requires accessing the current variables' value.

Petri nets presented in previous chapters are extended to incorporate data. A Petri net with Data is a Petri net with any number of variables (see Definitions 8.1 and 8.2 below). Petri nets with data can be seen as an abstracted version of high-level/colored Petri nets [50]. Colored Petri nets are extremely rich in expressiveness; however, many aspects are unimportant in our setting. Petri nets with data provide precisely the information needed for conformance checking of data-aware models and logs. In particular, the definitions presented is based on the work of de Leoni et al. [54].

Definition 8.1 (Variables and Values). $\mathcal{U}_{V N}$ is the universe of variable names. $\mathcal{U}_{V V}$ is the universe of values. $\mathcal{U}_{V M}=\mathcal{U}_{V N} \nrightarrow \mathcal{U}_{V V}$ is the universe of variable mappings. 
In this type of nets, transitions may read from and/or write to variables. Moreover, transitions are associated with guards over these variables, which define when these they can fire. A guard can be any formula over the process variables using relational operators $(<,>$ $,=)$ as well as logical operators such as conjunction $(\wedge)$, disjunction $(\vee)$, and negation $(\neg)$. A variable $v$ appear as $v_{r}$ or $v_{w}$, denoting the values read and written by the transition for $v$. We denote with Formulas $(V)$ the universe of such formulas defined over a set $V$ of variables. In the remainder, given a set $V \subset \mathcal{U}_{V N}$ of variable names, we denote $V_{R}=\left\{v_{r}: v \in V\right\}$ and $V_{W}=\left\{v_{w}: v \in V\right\}$.

Formally, a Petri net with Data (DPN) is defined as follows:

Definition 8.2 (Petri net with Data). A Petri net with Data $D P N=(S N, V$, val, init, read, write, guard) consists of

- a system net $S N=\left(P N, M_{\text {init }}, M_{\text {final }}\right)$ with $P N=(P, T, F, l)$,

- a set $V \subseteq \mathcal{U}_{V N}$ of data variables,

- a function val $\in V \rightarrow \mathcal{P}\left(\mathcal{U}_{V V}\right)$ that defines the values admissible for each variable, i.e., $\operatorname{val}(v)$ is the set of values that variable $v$ can have,

- a function init $\in V \rightarrow \mathcal{U}_{V V}$ that defines the initial value for each variable $v$ such that $\operatorname{init}(v) \in \operatorname{val}(v)$ (initial values are admissible),

- a read function read $\in T \rightarrow \mathcal{P}(V)$ that labels each transition with the set of variables that it reads,

- a write function write $\in T \rightarrow \mathcal{P}(V)$ that labels each transition with the set of variables that it writes,

- a guard function guard $\in T \rightarrow$ Formulas $\left(V_{W} \cup V_{R}\right)$ that associates a guard with each transition such that, for any $t \in T$ and for any $v \in V$, if $v_{r}$ appears in $\operatorname{guard}(t)$ then $v \in \operatorname{read}(t)$ and if $v_{w}$ appears in $\operatorname{guard}(t)$ then $v \in$ write $(t)$.

$\mathcal{U}_{D P N}$ is the universe of Petri nets with data.

The notion of bindings is essential for the remainder. A binding is a triplet $(t, r, w)$ describing the execution of transition $t$ while reading values $r$ and writing values $w$. A binding is valid if:

1. $r \in \operatorname{read}(t) \rightarrow \mathcal{U}_{V V}$ and $w \in$ write $(t) \rightarrow \mathcal{U}_{V V}$

2. for any $v \in \operatorname{read}(t): r(v) \in \operatorname{val}(v)$, i.e., all values read should be admissible,

3. for any $v \in$ write $(t): w(v) \in \operatorname{val}(v)$, i.e., all values written should be admissible.

4. Guard guard $(t)$ evaluates true. 


\begin{tabular}{|l|l|}
\hline Transition & Guard \\
\hline \hline Credit Request & true \\
\hline Verify & $0.1 \cdot A_{r}<I_{w}<0.2 \cdot A_{r}$ \\
\hline Assessment & $V_{R}=$ true \\
\hline Register Negative Verification & $V_{r}=$ false $\wedge D_{w}=$ false \\
\hline Inform Requester & true \\
\hline Renegotiate Request & $V_{r}=$ false $\wedge A_{w}<A_{r}$ \\
\hline Register Negative Request & $D_{r}=$ false \\
\hline Open Credit & $D_{r}=$ true \\
\hline
\end{tabular}

Table 8.1: Definitions of the guards of the transitions in Fig. 8.2. Variables and transition names are abbreviated as described in Figure 8.1. Subscripts $r$ and $w$ refer to, respectively, the values read and written for that given variable.

More specifically, let us introduce variable assignment $\left.\chi_{b}:\left(V_{R} \cup V_{W}\right) \not \rightarrow \mathcal{U}_{V V}\right)$ which is defined as follows: for any $v \in \operatorname{read}(t), \chi\left(v_{r}\right)=r(v)$ and, for any $v \in$ write $(t), \chi\left(v_{w}\right)=w(v)$. A binding $(t, r, w)$ makes $\operatorname{guard}(t)$ evaluate true if the evaluation of $\operatorname{guard}(t)$ wrt. $\chi_{b}$ returns true.

A marking $(M, s)$ of a Petri net with Data $D P N$ has two components: $M \in \mathcal{B}(P)$ is the control-flow marking and $s \in \mathcal{U}_{V M}$ with $\operatorname{dom}(s)=V$ and $s(v) \in \operatorname{val}(v)$ for all $v \in V$ is the data marking. The initial marking of a Petri net with Data $D P N$ is $\left(M_{\text {init }}\right.$, init $)$. Recall that init is a function that defines the initial value for each variable.

$(D P N,(M, s))[b\rangle$ denotes that a binding $b$ is enabled in marking $(M, s)$, which indicates that each of its input places $\bullet t$ contains at least one token (control-flow enabled), $b$ is valid and and $s \uparrow_{\operatorname{read}(t)}=r$ (the actual values read match the binding).

An enabled binding $b=(t, r, w)$ may occur, i.e., one token is removed from each of the input places $\bullet t$ and one token is produced for each of the output places $t \bullet$. Moreover, the variables are updated as specified by $w$. Formally: $M^{\prime}=(M \backslash \bullet t) \uplus t \bullet$ is the control-flow marking resulting from firing enabled transition $t$ in marking $M$ (abstracting from data) and $s^{\prime}=s \oplus w$ is the data marking where $s^{\prime}(v)=w(v)$ for all $v \in$ write $(t)$ and $s^{\prime}(v)=s(v)$ for all $v \in V \backslash$ write $(t)$. $(D P N,(M, s))[b\rangle\left(D P N,\left(M^{\prime}, s^{\prime}\right)\right)$ denotes that $b$ is enabled in $(M, s)$ and the occurrence of $b$ results in marking $\left(M^{\prime}, s^{\prime}\right)$.

Figure 8.2 shows a Petri net with Data $D P N_{e x}$ that models the same process as represented in Figure 8.1 as BPMN diagram, and Table 8.1 illustrates the conditions of the guards of the transitions of $D P N_{e x}$. The labeling function $l$ is such that the domain of $l$ is the set of transitions of $D P N_{e x}$ and, for each transition $t$ of $D P N_{e x}, l(t)=t$. In other words, the set of activity labels coincides with the set of transitions.

Let $\sigma_{b}=\left\langle b_{1}, b_{2}, \ldots, b_{n}\right\rangle$ be a sequence of bindings. $(D P N,(M, s))\left[\sigma_{b}\right\rangle\left(D P N,\left(M^{\prime}, s^{\prime}\right)\right)$ denotes that there is a set of markings $\left(M_{0}, s_{0}\right),\left(M_{1}, s_{1}\right), \ldots,\left(M_{n}, s_{n}\right)$ such that $\left(M_{0}, s_{0}\right)=$ $(M, s),\left(M_{n}, s_{n}\right)=\left(M^{\prime}, s^{\prime}\right)$, and $\left(D P N,\left(M_{i}, s_{i}\right)\right)\left[b_{i+1}\right\rangle\left(D P N,\left(M_{i+1}, s_{i+1}\right)\right)$ for $0 \leq i<n$. A 
marking $\left(M^{\prime}, s^{\prime}\right)$ is reachable from $(M, s)$ if there exists a $\sigma_{b}$ such that $(D P N,(M, s))\left[\sigma_{b}\right\rangle(D P N$, $\left.\left(M^{\prime}, s^{\prime}\right)\right)$.

$\phi_{f}(D P N)=\left\{\sigma_{b} \mid \exists_{s}\left(D P N,\left(M_{\text {init }}\right.\right.\right.$, init $\left.\left.)\right)\left[\sigma_{b}\right\rangle\left(D P N,\left(M_{\text {final }}, s\right)\right)\right\}$ is the set of complete binding sequences, thus describing the behavior of $D P N$.

Given a set of Petri nets with Data, the union is defined as the merge of those Petri nets.

Definition 8.3 (Union of Petri nets with Data). Let $D P N^{1}=\left(S N^{1}, V^{1}, v^{1} l^{1}\right.$, init $^{1}$, read $^{1}$, write $^{1}$, guard $\left.^{1}\right)$ and $D P N^{2}=\left(S N^{2}, V^{2}\right.$, val $^{2}$, init $^{2}$, read $^{2}$, write $^{2}$, guard $\left.^{2}\right)$ with $V^{1} \cap V^{2}=\emptyset$. $D P N^{1} \cup D P N^{2}=\left(S N^{1} \cup S N^{2}, V^{1} \cup V^{2}\right.$, val $^{1} \oplus$ val $^{2}$, init $^{1} \oplus$ init $^{2}$, read $^{3}$, write $^{3}$, guard $\left.^{3}\right)$ is the union such that

- $\operatorname{read}^{3}(t)=\operatorname{read}^{1}(t)$, write $^{3}(t)=$ write $^{1}(t)$, and $\operatorname{guard}^{3}(t)=\operatorname{guard}^{1}(t)$ if $t \in T^{1} \backslash T^{2}$,

- $\operatorname{read}^{3}(t)=\operatorname{read}^{2}(t)$, write $^{3}(t)=$ write $^{2}(t)$, and $\operatorname{guard}^{3}(t)=\operatorname{guard}^{2}(t)$ if $t \in T^{2} \backslash T^{1}$, and

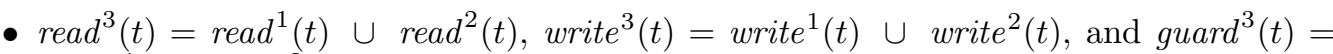
$\operatorname{guard}^{1}(t) \cdot \operatorname{guard}^{2}(t)$ if $t \in T^{1} \cap T^{2}$.

\subsubsection{Event Logs and Relating Models to Event Logs}

Next we extend the definition of event logs presented in Chapter 2 to incorporate data and relate them to the observable behavior of a $D P N$.

Definition 8.4 (Trace, Event Log with Data). A trace $\sigma \in\left(\mathcal{U}_{A} \times \mathcal{U}_{V M} \times \mathcal{U}_{V M}\right)^{*}$ is a sequence of activities with input and output data. $L \in \mathcal{B}\left(\left(\mathcal{U}_{A} \times \mathcal{U}_{V M} \times \mathcal{U}_{V M}\right)^{*}\right)$ is an event log with read and write information, i.e., a multiset of traces with data.

Definition 8.5 (From Bindings to Traces). Consider a Petri net with Data with transitions $T$ and labeling function $l \in T \nrightarrow \rightarrow \mathcal{U}_{A}$. A binding sequence $\sigma_{b} \in\left(T \times \mathcal{U}_{V M} \times \mathcal{U}_{V M}\right)^{*}$ can be converted into a trace $\sigma_{v} \in\left(\mathcal{U}_{A} \times \mathcal{U}_{V M} \times \mathcal{U}_{V M}\right)^{*}$ by removing the bindings that correspond to unlabeled transitions and by mapping the labeled transitions onto their corresponding label. $l\left(\sigma_{b}\right)$ denotes the corresponding trace $\sigma_{v}$.

Note that we overload the labeling function to binding sequences, $\sigma_{v}=l\left(\sigma_{b}\right)$. This is used to define $\phi(D P N)$ : the set of all visible traces.

Definition 8.6 (Observable Behavior of a Petri net with Data). Let $D P N$ be a Petri net with Data. $(D P N,(M, s))\left[\sigma_{v} \triangleright\left(D P N,\left(M^{\prime}, s^{\prime}\right)\right)\right.$ if and only if there is a sequence $\sigma_{b}$ such that $(D P N,(M, s))\left[\sigma_{b}\right\rangle\left(D P N,\left(M^{\prime}, s^{\prime}\right)\right)$ and $\sigma_{v}=l\left(\sigma_{b}\right) . \phi(D P N)=\left\{l\left(\sigma_{b}\right) \mid \sigma_{b} \in \phi_{f}(D P N)\right\}$ is the set of visible traces starting in $\left(M_{\text {init }}\right.$, init $)$ and ending in $\left(M_{\text {final }}, s\right)$ for some data marking $s$.

Definition 8.7 (Perfectly Fitting with Data). A trace $\sigma \in\left(\mathcal{U}_{A} \times \mathcal{U}_{V M} \times \mathcal{U}_{V M}\right)^{*}$ is perfectly fitting $D P N \in \mathcal{U}_{D P N}$ if $\sigma \in \phi(D P N)$. An event $\log L \in \mathcal{B}\left(\left(\mathcal{U}_{A} \times \mathcal{U}_{V M} \times \mathcal{U}_{V M}\right)^{*}\right)$ is perfectly fitting $D P N$ if all of its traces are perfectly fitting. 
Later, we will need to project binding sequences and traces onto subsets of transitions/activities and variables. Therefore, we introduce a generic projection operator $\Pi_{Y, V}(\sigma)$ that removes transitions/activities not in $Y$ and variables not in $V$.

Definition 8.8 (Projection). Let $X$ be a set of transitions or activities (i.e., $X \subseteq T$ or $\left.X \subseteq \mathcal{U}_{A}\right)$. Let $Y \subseteq X$ be a subset and $V \subseteq \mathcal{U}_{V N}$ a subset of variable names. Let $\sigma \in(X \times$ $\left.\mathcal{U}_{V M} \times \mathcal{U}_{V M}\right)^{*}$ be a binding sequence or a trace with data. $\Pi_{Y, V}(\sigma) \in\left(Y \times\left(V \not \rightarrow \mathcal{U}_{V V}\right) \times(V \not \rightarrow\right.$ $\left.\left.\mathcal{U}_{V V}\right)\right)^{*}$ is the projection of $\sigma$ onto transitions/activities $Y$ and variables $V$. Bindings/events unrelated to transitions/activities in $Y$ are removed completely. Moreover, for the remaining bindings/events all read and write variables not in $V$ are removed. $\Pi_{Y, V}(L)=\left[\Pi_{Y, V}(\sigma) \mid\right.$ $\sigma \in L]$ lifts the projection operator to the level of logs.

\subsubsection{Data Alignments}

In this section we extend the alignment theory presented in Chapters 5 and 6 to incorporate the notion of data. Alignments shows how the event log can be replayed on the process model, and they are composed by sequences of moves:

Definition 8.9 (Legal alignment moves). Let $D P N=(S N, V$, val, init, read,write,guard $)$ be a Petri net with Data, with $S N=\left(P N, M_{\text {init }}, M_{\text {final }}\right)$ and $P N=$ $(P, T, F, l)$. Let $S_{L}=\mathcal{U}_{A} \times \mathcal{U}_{V M} \times \mathcal{U}_{V M}$ be the universe of events. Let $S_{D P N}=T \times \mathcal{U}_{V M} \times \mathcal{U}_{V M}$ be the universe of bindings of $D P N$. Let be $S_{D P N}^{\gg}=S_{D P N} \cup\{\gg\}$ and $S_{L}^{\gg}=S_{L} \cup\{\gg\}$.

A legal move in an alignment is represented by a pair $\left(s_{L}, s_{M}\right) \in\left(S_{L}^{\gg} \times S_{D P N}^{\gg}\right) \backslash\{(\gg, \gg)\}$ such that

- $\left(s_{L}, s_{M}\right)$ is a move in $\log$ if $s_{L} \in S_{L}$ and $s_{M}=\gg$,

- $\left(s_{L}, s_{M}\right)$ is a move in model if $s_{L}=\gg$ and $s_{M} \in S_{D P N}$,

- $\left(s_{L}, s_{M}\right)$ is a move in both without incorrect read/write operations if $s_{M}=(t, r, w) \in$ $S_{D P N}$ and $s_{L}=(l(t), r, w) \in S_{L}$,

- $\left(s_{L}, s_{M}\right)$ is a move in both with incorrect read/write operations if $s_{M}=(t, r, w) \in S_{D P N}$ and $s_{L}=\left(l(t), r^{\prime}, w^{\prime}\right) \in S_{L}$, and $r \neq r^{\prime}$ or $w \neq w^{\prime}$.

All other moves are considered as illegal.

Definition 8.10 (Data Alignments). Let $D P N=(S N, V$, val, init, read,write, guard) be a Petri net with Data and $\sigma \in\left(S_{L}\right)^{*}$ be an event-log trace. Let $\mathcal{A}_{D P N}$ be the set of legal moves for $D P N$. A complete alignment of $\sigma_{L}$ and $D P N$ is a sequence $\gamma \in \mathcal{A}_{D P N}{ }^{*}$ such that, ignoring all occurrences of $\gg$, the projection on the first element yields $\sigma_{L}$ and the projection on the second yields a $\sigma_{P} \in \phi_{f}(D P N)$.

Table 8.2 shows two complete alignments of the process model in Figure 8.2 and the log trace $\sigma_{e x}$ from Section 1 . 


\begin{tabular}{|l|l|}
\hline Event-Log Trace & Process \\
\hline$(\mathbf{a},\{(\mathrm{A}, 4000)\})$ & $(\mathbf{a},\{(\mathrm{A}, 4000)\})$ \\
$(\mathbf{b},\{(\mathrm{I}, 450),(\mathrm{V}$, false $)\})$ & $(\mathbf{b},\{(\mathrm{I}, 450),(\mathrm{V}$, true $)\})$ \\
$(\mathbf{c},\{(\mathrm{D}$, true $)\})$ & $(\mathbf{c},\{(\mathrm{D}$, true $)\})$ \\
$(\mathbf{e}, \emptyset)$ & $(\mathbf{e}, \emptyset)$ \\
$(\mathbf{f},\{(\mathrm{A}, 5000)\})$ & $(\mathbf{f},\{(\mathrm{A}, 3000)\})$ \\
$(\mathbf{b},\{(\mathrm{I}, 450),(\mathrm{V}$, false $)\})$ & $(\mathbf{b},\{(\mathrm{I}, 450),(\mathrm{V}$, false $)\})$ \\
$(\mathbf{d},\{(\mathrm{D}$, false $)\})$ & $(\mathbf{d},\{(\mathrm{D}$, false $)\})$ \\
$(\mathbf{e}, \emptyset)$ & $(\mathbf{e}, \emptyset)$ \\
$(\mathbf{h}, \emptyset)$ & $\gg$ \\
$\gg$ & $(\mathbf{g}, \emptyset)$ \\
\hline
\end{tabular}

(a)

\begin{tabular}{|l|l|}
\hline Event-Log Trace & Process \\
\hline$(\mathbf{a},\{(\mathrm{A}, 4000)\})$ & $(\mathbf{a},\{(\mathrm{A}, 5100)\})$ \\
$(\mathbf{b},\{(\mathrm{I}, 450),(\mathrm{V}, \mathrm{false})\})$ & $(\mathbf{b},\{(\mathrm{I}, 511),(\mathrm{V}, \mathrm{true})\})$ \\
$(\mathbf{c},\{(\mathrm{D}$, true $)\})$ & $(\mathbf{c},\{(\mathrm{D}$, true $)\})$ \\
$(\mathbf{e}, \emptyset)$ & $(\mathbf{e}, \emptyset)$ \\
$(\mathbf{f},\{(\mathrm{A}, 5000)\})$ & $(\mathbf{f},\{(\mathrm{A}, 5000)\})$ \\
$(\mathbf{b},\{(\mathrm{I}, 450),(\mathrm{V}$, false $)\})$ & $(\mathbf{b},\{(\mathrm{I}, 511),(\mathrm{V}, \mathrm{false})\})$ \\
$(\mathbf{d},\{(\mathrm{D}$, false $)\})$ & $(\mathbf{d},\{(\mathrm{D}, \mathrm{false})\})$ \\
$(\mathbf{e}, \emptyset)$ & $(\mathbf{e}, \emptyset)$ \\
$(\mathbf{h}, \emptyset)$ & $\gg$ \\
$\gg$ & $(\mathbf{g}, \emptyset)$ \\
\hline
\end{tabular}

(b)

Table 8.2: Examples of complete alignments of $\sigma_{\text {example }}$ and $N$. For readability, the read operations are omitted. Of course, read operations for any variable must match the most recent value for that variable. Any move is highlighted with a gray color if it contains deviations, i.e. it is not a move in both without incorrect read/write operations.

As it is explained in Chapter 5, in order to define the severity of a deviation, we introduce a cost function on legal moves: $\kappa \in \mathcal{A}_{D P N} \rightarrow \mathbb{R}_{0}^{+}$. This cost function can be used to favor one type of explanation for deviations over others. The cost of each legal move depends on the specific model and process domain and, hence, the cost function $\kappa$ needs to be defined specifically for each setting. The cost of an alignment $\gamma$ is the sum of the cost of all individual moves composing it: $\mathcal{K}(\gamma)=\sum_{\left(s_{L}, s_{M}\right) \in \gamma} \kappa\left(s_{L}, s_{M}\right)$.

However, we do not aim to find just any complete alignment. Our goal is to find a complete alignment of $\sigma_{L}$ and $D P N$ which minimizes the cost: an optimal alignment. Let $\Gamma_{\sigma_{L}, N}$ be the (infinite)set of all complete alignments of $\sigma_{L}$ and $D P N$. The alignment $\gamma \in \Gamma_{\sigma_{L}, D P N}$ is an optimal alignment if, for all $\gamma^{\prime} \in \Gamma_{\sigma_{L}, N}, \mathcal{K}(\gamma) \leq \mathcal{K}\left(\gamma^{\prime}\right)$. Note that an optimal alignment does not need to be unique, i.e. multiple complete alignments with the same minimal cost may exist.

Let us consider again our example introduced above. Let us assume to have a cost function $\kappa^{s}$ such that $\kappa^{s}\left(s_{L}, s_{M}\right)=1$ if $\left(s_{L}, s_{M}\right)$ is a visible move in process or a move in log (i.e. $s_{L}=\gg$ and $s_{M}$ corresponds to a labeled transition or, conversely, $s_{M}=\gg$, respectively) or a move in both with incorrect read/write operations and $\kappa^{s}\left(s_{L}, s_{M}\right)=0$ in case of move in both without incorrect read/write operations or a move in model corresponding to an unlabeled transition. The alignment in Table 8.2a has a cost of 6 whereas the alignment in Table $8.2 \mathrm{~b}$ has a cost $8 .^{2}$ It follows that the former is a better alignment. As a matter of fact, it is also an optimal alignment, although it is not the only one. For instance, any variation of such an alignment where the move for $f$ is of the form (now including read operations) $((\mathbf{f},\{(A, 4000)\},\{(A, 5000)\})(\mathbf{f},\{(A, 4000)\},\{(A, x)\})\}))$ with $2250<x<4000$ corresponds to an optimal alignment, as well.

\footnotetext{
${ }^{2}$ They also include a cost of two that is accounted for incorrect read operations, not shown in the alignments, which are caused by incorrect write operations.
} 
As we have mentioned, the data-aware conformance checking is undecidable in the general case. This is caused by the fact that Petri nets with Data are Turing-complete. Therefore, it is not decidable to verify whether a sequence of valid bindings exists that takes from the initial marking to any final marking $\left(M_{\text {final }}, s\right)$. As a consequence, for instance, it is not possible to find an alignment of a Petri net with Data and the empty log trace. However, the problem becomes decidable (with an exponential complexity) if guards are restricted to linear (in)equalities.

\subsection{Valid Decomposition of Data-aware Models}

In Chapter 6 a valid decomposition [1] is presented in terms of Petri nets: the overall system net $S N$ is decomposed into a collection of subnets $\left\{S N^{1}, S N^{2}, \ldots, S N^{n}\right\}$ such that the union of these subnets yields the original system net. A decomposition is valid if the subnets "agree" on the original labeling function (i.e., the same transition always has the same label), each place resides in just one subnet, and also each invisible transition resides in just one subnet. Moreover, if there are multiple transitions with the same label, they should reside in the same subnet.

As shown in Chapter 6, these observations imply that conformance checking can be decomposed. Any trace that fits the overall process model can be decomposed into smaller traces that fit the individual model fragments. Moreover, if the smaller traces fit the individual fragments, then they can be composed into a trace that fits into the overall process model. This result is the basis for decomposing process mining problems.

In this chapter, the definition of valid decomposition is extended to cover Petri nets with data.

Definition 8.11 (Valid Decomposition for Petri nets with Data). Let $D P N \in \mathcal{U}_{D P N}$ be a Petri net with Data. $D=\left\{D P N^{1}, D P N^{2}, \ldots, D P N^{n}\right\} \subseteq \mathcal{U}_{D P N}$ is a valid decomposition if and only if:

- for all $1 \leq i \leq n$ : $D P N^{i}=\left(S N^{i}, V^{i}\right.$, val $^{i}$, init $^{i}$, read $^{i}$, write $^{i}$, guard $\left.^{i}\right)$ is a Petri net with Data, $S N^{i}=\left(P N^{i}, M_{\text {init }}^{i}, M_{\text {final }}^{i}\right) \in \mathcal{U}_{S N}$ is a system net, and $P N^{i}=\left(P^{i}, T^{i}, F^{i}, l^{i}\right)$ is a labeled Petri net,

- $D^{\prime}=\left\{S N^{1}, S N^{2}, \ldots, S N^{n}\right\} \subseteq \mathcal{U}_{S N}$ is a valid decomposition of $\bigcup_{1 \leq i \leq n} S N^{i}$,

- $V^{i} \cap V^{j}=\emptyset$ for $1 \leq i<j \leq n$,

- $D P N=\bigcup_{1 \leq i \leq n} D P N^{i}$.

$\mathcal{D}(D P N)$ is the set of all valid decompositions of $D P N$.

Each variable appears in precisely one of the subnets. Therefore, $V^{i} \cap V^{j}=\emptyset$ implies that there cannot be two fragments that read and or write the same data variables: 
$\bigcup_{t \in T^{i}}$ read $^{i}(t) \cup$ write $^{i}(t) \bigcap \bigcup_{t \in T^{j}} \operatorname{read}^{j}(t) \cup$ write $^{j}(t)=\emptyset$ for $1 \leq i<j \leq n$. Moreover, two guards in different fragments cannot refer to the same variable. If a transition $t$ appears in multiple fragments, then it needs to have a visible unique label. Such a uniquely labeled transition $t$ shared among fragments, may use, read, or write different variables in different fragments. Since $D P N=\bigcup_{1 \leq i \leq n} D P N^{i}$, we know that, for all $t$ in $D P N$, guard $(t)$ is the product of all guard $^{i}(t)$ such that $t \in T^{i}$. Without loss of generality we can assume that the first $k$ fragments share $t$. Hence, $\operatorname{guard}(t)=\operatorname{guard}^{1}(t) \cdot \ldots \cdot \operatorname{guard}^{k}(t)$. Hence, in a valid decomposition, the guard of a shared transition can only be split if the different parts do not depend on one another. Notice that, the splitting of the data variables is limited by how the variables are used throughout the process, existing a worst-case where all the data variables are used in all the steps of the process.

Based on these observations, we prove that we can decompose conformance checking also for Petri nets with data.

Theorem 8.12 (Conformance Checking With Data Can be Decomposed). Let $L \in \mathcal{B}\left(\left(\mathcal{U}_{A} \times\right.\right.$ $\left.\left.\mathcal{U}_{V M} \times \mathcal{U}_{V M}\right)^{*}\right)$ be an event log with information about reads and writes and let $D P N \in \mathcal{U}_{D P N}$ be a Petri net with Data. For any valid decomposition $D=\left\{D P N^{1}, D P N^{2}, \ldots, D P N^{n}\right\} \subseteq$ $\mathcal{U}_{D P N}: L$ is perfectly fitting Petri net with Data $D P N$ if and only if for all $1 \leq i \leq n$ : $\Pi_{A_{v}\left(S N^{i}\right), V^{i}}(L)$ is perfectly fitting $D P N^{i}$.

Proof. Let $D P N=(S N, V$, val, init, read, write, guard $)$ be a Petri net with Data with $S N=$ $\left(P N, M_{\text {init }}, M_{\text {final }}\right)$ and $P N=(P, T, F, l)$. Let $D=\left\{D P N^{1}, D P N^{2}, \ldots D P N^{n}\right\}$ be a valid decomposition of $D P N$ with $D P N^{i}=\left(S N^{i}, V^{i}\right.$, val $^{i}$, init $^{i}$, read $^{i}$, write $^{i}$, guard $\left.^{i}\right), S N^{i}=$ $\left(P N^{i}, M_{\text {init }}^{i}, M_{\text {final }}^{i}\right) \in \mathcal{U}_{S N}$, and $P N^{i}=\left(P^{i}, T^{i}, F^{i}, l^{i}\right)$.

$(\Rightarrow)$ Let $\sigma_{v} \in L$ be such that there exists a data marking $s$ such that $\left(D P N,\left(M_{i n i t}\right.\right.$, init $))\left[\sigma_{v} \triangleright\left(D P N,\left(M_{\text {final }}, s\right)\right)\right.$. This implies that there exists a corresponding $\sigma_{b}$ with $(D P N$, $\left(M_{\text {init }}\right.$, init $\left.)\right)\left[\sigma_{b}\right\rangle\left(D P N,\left(M_{\text {final }}, s\right)\right)$ and $l\left(\sigma_{b}\right)=\sigma_{v}$. For all $1 \leq i \leq n$, we need to prove that there is a $\sigma_{b}^{i}$ with $\left(D P N^{i},\left(M_{\text {init }}^{i}, i n i t^{i}\right)\right)\left[\sigma_{b}^{i}\right\rangle\left(D P N^{i},\left(M_{\text {final }}^{i}, \bar{s}^{i}\right)\right)$ for some $s^{i}$. This follows trivially because $D P N^{i}$ can mimic any move of $D P N$ with respect to transitions $T^{i}$ : just take $\sigma_{b}^{i}=\Pi_{T^{i}, V^{i}}\left(\sigma_{b}\right)$. Note that guards can only become weaker by projection.

$(\Leftarrow)$ Let $\sigma_{v} \in L$. For all $1 \leq i \leq n$, let $\sigma_{b}^{i}$ be such that $\left(D P N^{i},\left(M_{\text {init }}^{i}\right.\right.$, init $\left.\left.^{i}\right)\right)\left[\sigma_{b}^{i}\right\rangle$ $\left(D P N^{i},\left(M_{\text {final }}^{i}, s^{i}\right)\right)$ and $l^{i}\left(\sigma_{b}^{i}\right)=\Pi_{A_{v}\left(S N^{i}\right), V^{i}}\left(\sigma_{v}\right)$. The different $\sigma_{b}^{i}$ sequences can be stitched together into an overall $\sigma_{b}$ s.t. $\left(D P N,\left(M_{\text {init }}\right.\right.$, init $\left.)\right)\left[\sigma_{b}\right\rangle\left(D P N,\left(M_{\text {final }}, s\right)\right)$ with $s=s^{1} \oplus s^{2} \oplus$ $\ldots \oplus s^{n}$. This is possible because transitions in one subnet can only influence other subnets through unique visible transitions and these can only move synchronously as defined by $\sigma_{v}$. Moreover, guards can only be split in independent parts (see Definition 8.11). Suppose that $t$ appears in $T_{i}$ and $T_{j}$, then $\operatorname{guard}(t)=\operatorname{guard}^{i}(t) \cdot \operatorname{guard}^{j}(t)$. Hence, a read/write in subnet $i$ cannot limit a read/write in subnet $j$. Therefore, we can construct $\sigma_{b}$ and $l\left(\sigma_{b}\right)=\sigma_{v}$. 


\subsection{SESE-based Strategy for a Valid Decomposition}

In this section we present a concrete strategy to instantiate the valid decomposition definition over a Petri net with data presented in the previous section (cf. Definition8.11). Similar to Chapter 6, the proposed strategy decomposes the Petri net with data in a number of SingleEntry Single-Exit (SESE) components, creating meaningful fragments of a process model [72, 66]. SESE decomposition is indicated for well-structured models, whereas for unstructured models some automatic transformation techniques can be considered as a pre-processing step $[42]$.

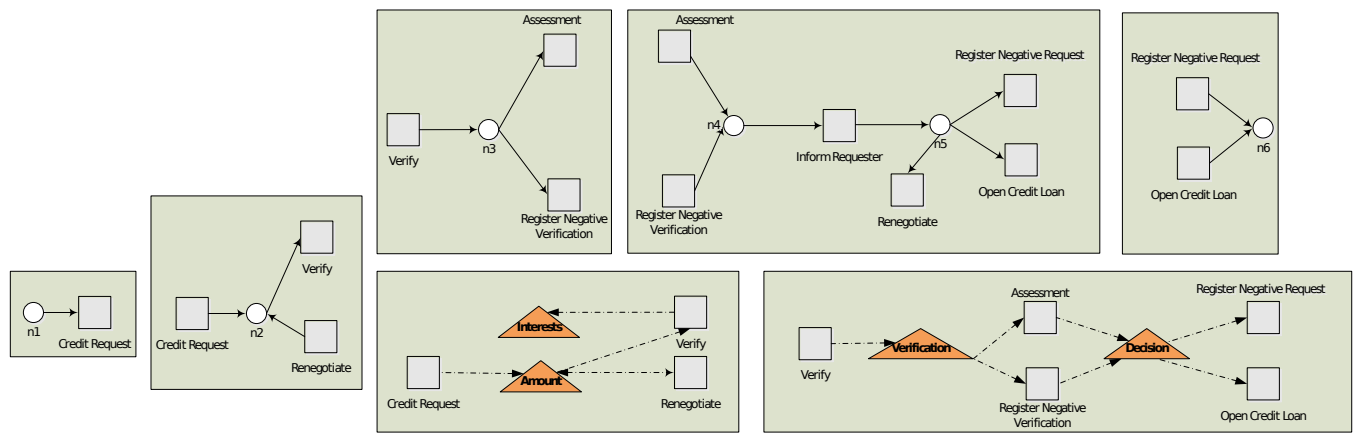

Figure 8.3: SESE-based decomposition for the running example, with 2-decomposition.

To extend the SESE decomposition strategy presented in Chapter 6 to also account for data, one simply considers its application over the data workflow graph, an extension of the workflow graph where the variables and read/write arcs are also included.

Definition 8.13 (Data Workflow Graph). The data workflow graph of a Petri net with Data $\left(\left((P, T, F, l), M_{\text {init }}, M_{\text {final }}\right), V\right.$, val, init, read, write, guard $)$ with data arcs $R=\{(v, t) \mid v \in$ $\operatorname{read}(t)\}$ and $W=\{(t, v) \mid v \in$ write $(t)\}$ is the workflow graph $D W G=(S, E)$ with $S=$ $P \cup T \cup V$ and $E=F \cup R \cup W$.

The SESE decomposition proposed to analyze the conformance of Petri nets with data, is similar to the one presented in Chapter 6 but considering data workflow graph instead. Algorithm 8.1 describes the steps necessary to construct a SESE decomposition. The arcs are partitioned in SESEs by means of creating the RPST from the data workflow graph, and selecting a particular set of SESES over it. Once the partitioning is done, a subnet is created for each part. Subnets contradicting some of the requirements of Definition 8.11 (e.g. sharing places, invisible or duplicate transitions, variables, or transitions with non-splitting guards) are merged to preserve the valid decomposition definition.

Figure 8.3 shows the decomposition for the example of Figure 8.2, where the RPST is partitioned using the 2-decomposition algorithm (cf. Chapter 6), i.e., SESEs of at most 2 


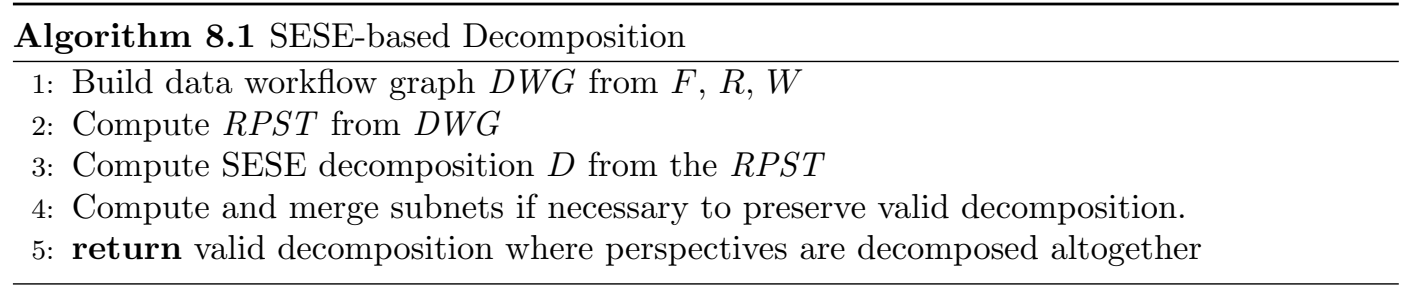

$\operatorname{arcs}^{3}$. To ensure a valid decomposition is obtained, step 4 of Algorithm 8.1 combines multiple SESE fragments into larger fragments, which are not necessarily SESEs anymore.

\subsection{Implementation and Experimental Results}

The approach discussed in this chapter has been implemented as a plug-in for the open-source ProM framework for process mining.

The plug-in requires a Petri Net with Data and an event log as input and returns as many bags of alignments as the number of fragments in which the Petri Net with Data has been decomposed. Each bag refers to a different fragment and shows the alignments of each $\log$ trace and that fragment. A second type of output is also produced in which the alignments' information is projected onto the Petri net with Data. Transitions are colored according to the number of deviations: if no deviation occurs for a given transition, the respective box in the model is white-colored. The filling color of a box shades towards red as a larger fraction of deviations occur for the corresponding transition. Something similar is also done for variables: the more incorrect read/write operations occur for a variable, the more the variable is shown with a color close to red. This output is extremely interesting from an end-user viewpoint as it allows for gaining a helicopter view on the main causes of deviations [54].

The plug-in has been evaluated using a number of synthetic event logs and also a real-life process. The plug-in has been evaluated using the model in Figure 8.2 and with a number of event $\operatorname{logs}$ that were artificially generated. In particular, we have generated different event logs with the same number of traces, 5000, but increasing number of events, meaning that, on average, traces were of different length. To simulate that, for each simulated process execution, an increasing number of renegotiations was enforced to happen. Traces were also generated so as to contain a number of deviations: the event logs were generated in a way that $25 \%$ of transitions fired violating the guards.

Figure 8.4 shows the results of checking for conformance of the different event logs and the process model, comparing the SESE-based decomposition with $k=2$ with the case in which no decomposition is made. To check the conformance of each fragment, we used the

\footnotetext{
${ }^{3}$ Although the SESEs have at most two arcs, this is not guaranteed for the final subnets, i.e., some subnets are merged to preserve the valid decomposition definition.
} 


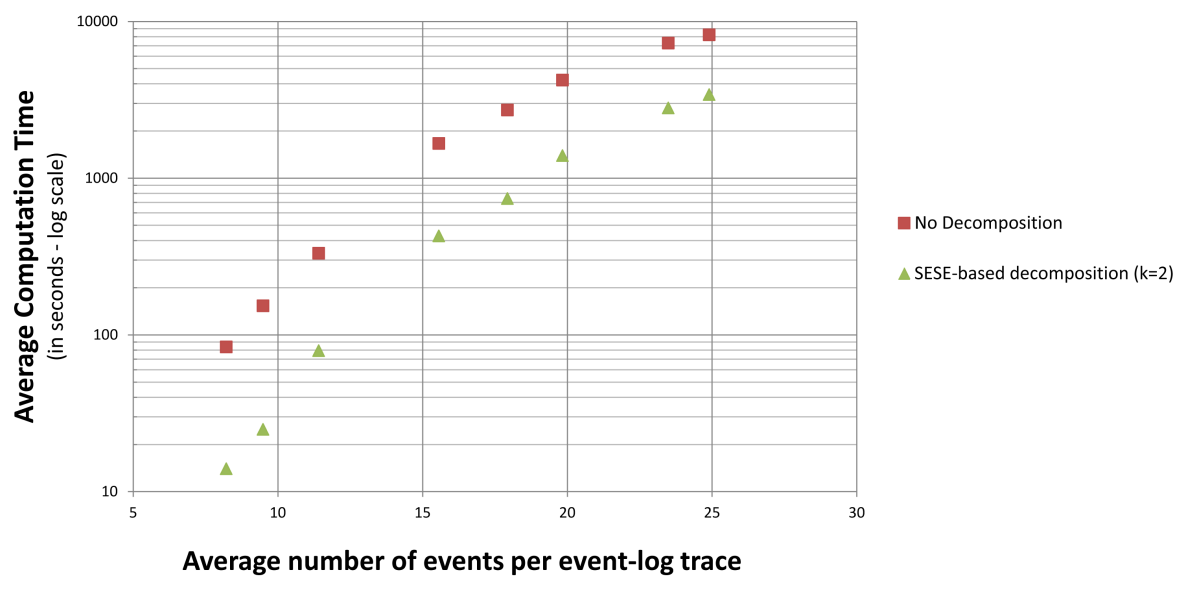

Figure 8.4: Computation time for checking the conformance of the Petri net with Data in Figure 8.2 and event logs of different size. The $\mathrm{Y}$ axis is on a logarithmic scale.

technique reported in [54]. Each dot in the chart indicates a different event log with traces of different size. The computation time refers to the conformance checking of the whole event logs (i.e., 5000 traces). The decomposed net is the same as in Figure 8.3. Regarding the cost function, we assign cost 1 to any deviation; however, this could be customized based on domain knowledge. The results show that, for every combination of event log and process model, the decomposition significantly reduces the computation time and the improvement is exponential in the size of the event log.

To assess the practical relevant of the approach, we also performed an evaluation with a Dutch financial institute. The process model was provided by a process analyst of the institute and consists of 21 transitions: 13 transitions with unique labels, 3 activities labels shared between 2 transitions (i.e. 6 transitions in total), plus 3 invisible transitions. The model contains twelve process variables, which are read and written by the activities when being executed. We were also provided with an event log that recorded the execution of 111 real instances of such a process; overall, the 111 log traces contained 3285 events, which means roughly 29.6 events per trace. We checked the conformance of this process model and this event log, comparing the results when the model has or has not been decomposed in small fragments. For conformance checking, here we used the technique reported in [57] since the provided process model breaks the soundness assumptions required by [54]. For this experiment round, the additional optimizations proposed in [57] were deactivated to allow for a fair comparison.

The application of the decomposition approach to this real-life case study has shown tremendous results: the conformance checking has required 52.94 seconds when the process model was decomposed using the SESE-based technique presented in Section 8.4; conversely, 
it required 52891 seconds when the model was not decomposed. This indicates that decomposing the process model allowed us to save $99.999 \%$ of the computation time. As a matter of fact, we tried for different values of SESE parameter $k$ but we obtained similar results: the computation time did not move away for more than 1 second. The reason of this is related to the fact that every decomposition for any value of $k$ always contained a certain fragment, along with others. Indeed, that fragment could not be decomposed any further than a given extent. Since the computation time was mostly due to constructing alignments with that fragment, no significant difference in computation time could be observed when varying $k$.

\subsection{Conclusions}

Checking conformance between an event log and a process model is known to be a complex procedure. That complexity explodes even more when we consider data-aware conformance checking with multi-perspective models. This chapter proposed the extension of control-flow decomposed conformance checking techniques to alleviate the computation time of dataaware processes. The decomposition definition proposed is proven to be correct from a conformance point of view, and the experiments showed a significant reduction in time with respect to the monolithic approach. In the future, new decomposition strategies may be considered, and the proposed techniques may be extended to other conformance dimensions, such as precision. 



\section{Chapter 9}

\section{Event-based Real-time Decomposed Conformance Checking}

\subsection{Introduction}

In this chapter we use the application of decomposed techniques to check conformance in event-based real-time systems. Unlike forensic conformance checking, where conformance is analyzed a posteriori once the case has finished, real-time techniques check conformance on the fly. Real-time checking techniques are specially indicated for monitoring, immediate fraud detection and governance, risk and compliance verification, and failure protection.

Although both forensic and real-time checking analyze the conformance between models and reality, the latter presents a list of new challenges for its applicability. First, the proposed approach should consider the computation time a priority. The analysis must be conducted on a regular basis and the results must be output in a short period of time. Second, given the periodicity of the analysis and the urge of the conclusions, the real-time approaches must focus on the fine-grained localization of the deviation and the understanding of their causes. Finally, real-time systems must be event-based instead of trace-based, i.e., the analysis must not require a complete trace in other to detect possible mismatches.

In this chapter we propose decomposed conformance analysis methodology to support the real-time monitoring of event-based data streams, which aims to provide an answer to

- The materials reported in this chapter are partially published in [29] - 
the challenges listed above, being more efficient than related approaches and able to localize deviations in a more fine-grained manner. The remainder of this chapter is organized as follows. Section 9.2 presents the methodology proposed, that combines both decomposition and event-based heuristic replay. Section 9.3 presents an extensive example case to illustrate the benefits of the methodology, and an experimental comparison with related approaches. Finally, Section 9.4 concludes the chapter.

\subsection{Event-based Real-time Decomposed Conformance}

In this section we present the proposed methodology, that combines: 1) a decomposition of the model, 2) with an event-based heuristic replay of the log. Figure 9.1 provides a schematic overview of the approach, where each event is dispatched to its corresponding component to be replayed.

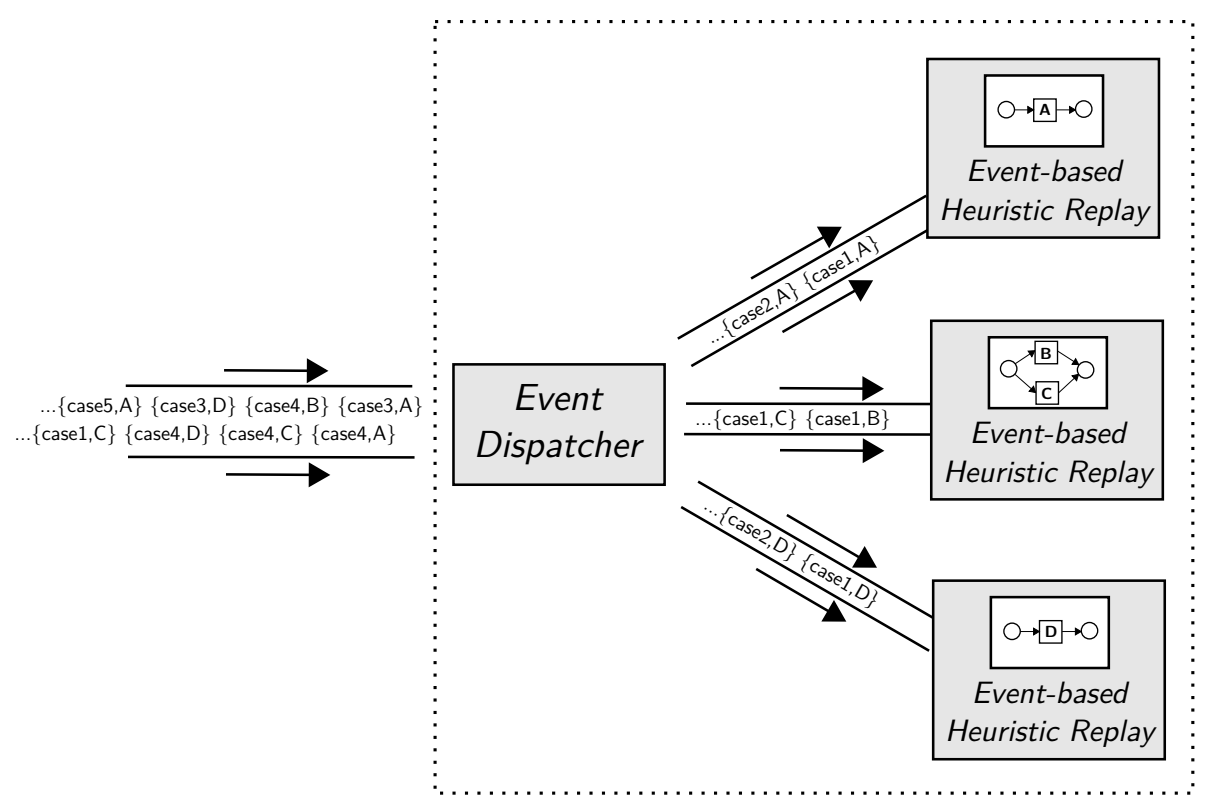

Figure 9.1: Architectural overview of the developed real-time decomposed conformance checking technique.

\subsubsection{Model and Log Decomposition}

The first phase of the proposed methodology entails decomposition. Formally, the overall system net $S N$ is broken down into a collection of subnets $\left\{S N^{1}, S N^{2}, \ldots S N^{n}\right\}$ such that 
the union of these subnets yields the original system net $S N$. By means of decomposing the original model into a set of subnets we aim to achieve the following goals. First, fragment the conformance problems into a set of more comprehensive semantic elements aiding on the diagnosis. Second, restrict the possible pernicious effects of the heuristics decisions taken during the conformance analysis (see Phase 3 below). Third, speed-up the analysis compared with non-decomposed conformance checking techniques.

Due to the final goal of analyzing conformance, not all possible decomposition approaches are appropriate for this task. Only those valid decompositions that preserve the conformance integrity should be considered (cf. Chapter 6). That is, given the original net and the decomposed version, the original net perfectly conforms iff all the subnets in the decomposed setting perfectly conforms. In other words, no conformance anomalies should be lost or introduced in the transition from the overall model to the decomposed one. As it is presented in Chapter 6, a valid decomposition - applicable on Petri nets - is defined informally as the decomposition that satisfies the following conditions:

1. Each arc of the overall net belongs to exactly one of the subnets.

2. Each place of the overall net belongs to exactly one of the subnets.

3. Invisible transitions appears in precisely one of the subnets.

4. Visible, duplicate transitions appear in precisely one of the subnets.

5. Visible, non-duplicate transitions may appear in multiple subnet.

In other words, all elements in the original Petri net model must belong to a subnet, but only visible, non-duplicate transitions can be shared among several subnet.

As it is mentioned in previous chapters, there exist several possible valid decomposition strategies: minimal, passages, SESEs, etc. In this methodology we consider a valid decomposition based on the SESE decomposition proposed in Chapter 6, i.e. subgraphs in the workflow graph defined over a system net having single entry and exit boundary nodes. SESEs perfectly reflect the idea of subprocesses within the main process, important to obtain a meaningful real-time decomposition. Figure 9.2 shows an example of SESE for the illustrative case shown in Section 9.3. The SESE decomposition can be combined with a user-supervised post-processing step where several SESEs are merged in order to obtain components that better fulfill the domain-aware monitoring.

Once a system net has been decomposed into a set of submodels, this collection of models is passed to a central event dispatcher, which also serves to listen for incoming events. For each submodels, it is examined whether it contains a transition $t$ which maps to the incoming event $e$. If it does, this indicates that the event at hand should be replayed on this particular submodel (multiple such submodels can be found), and the event is passed forward to this model fragment. 


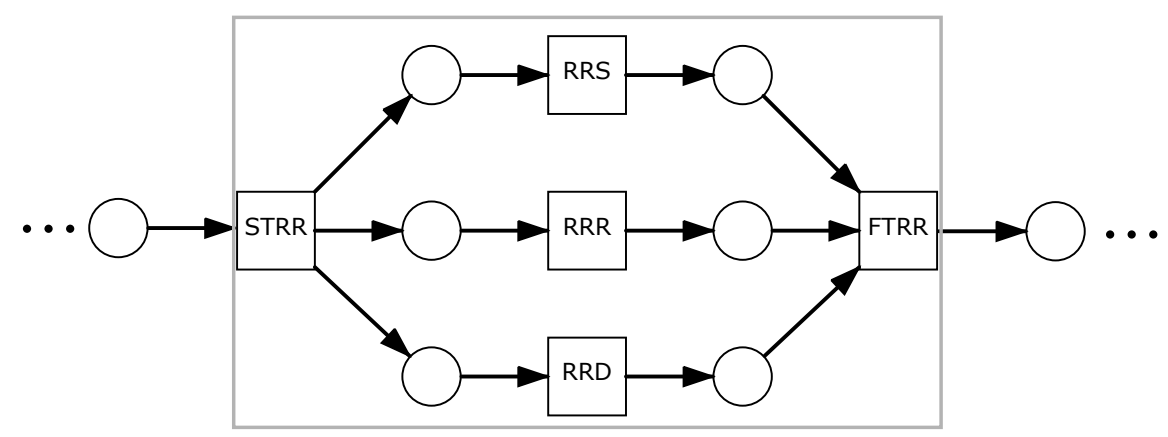

Figure 9.2: "Open and register transaction" SESE from the case example in Section 9.3. $S T R R$ and FTRR are the entry and exit boundary nodes of the SESE, respectively. The rest of places and transitions are interior nodes of the SESE.

\subsubsection{Event-based Heuristic Replay}

Once it is determined which process model fragment(s) should parse the incoming event, the actual replay of this event on each such fragment is performed. In previous chapters - Chapters 5 and 6 - we illustrate the use and benefits of a optimal conformance checking based on alignments. However, given the event-based nature of the methodology, and the need of efficient approaches in real-time system, in this chapter we propose the use of replay based conformance techniques. In the seminal work [80], a "fitness" metric is presented to describe the extent to which event traces can be associated with valid execution paths in the process model, and an "appropriateness" metric is proposed to asses whether the process model describes the observed behavior accurately enough. The aforementioned approach replays the traces of the $\log$ in the model to evaluate these metrics. One of the drawbacks of this approach is that for undeterministic models, the heuristics used in the replay may lead to overestimating the metrics, due to the artificial creation of superfluous tokens in the model. Several solutions have been proposed to overcome this issue. Weidlich et al. propose a system to check process model consistency based on "behavioral profiles" [99, 89] — which can be derived in a straightforward and efficient manner but with loss of some granularity regarding the exact traces which can be accepted by the model at hand.

In this chapter we propose the use of a replay algorithm based on the work of vanden Broucke et al. [30, 26]. The informal idea is the following: for each process model fragment, a state list is maintained denoting the current marking reached by the currently-running process instances. When an event is queued for replay by a process fragment, the state linked to process instance is progressed by investigating whether there exists an enabled transition for such activity. The outcome of this evaluation determines if the process model is showing discrepancies or not.

Some additional remarks should be provided at this point. First of all, we note that we 
apply a heuristic, event-granular replayer similar to the one applied in [30]. The reasoning behind the choice to opt for a replayer playing the token game instead of an alternative approach such as alignment or behavioral profile based techniques [16, 89, 99] are twofold. First, alignment and behavioral profile based replayers perform their analysis on a trace, rather than event level, meaning that a complete process instance needs to finalize in order to align the log trace with a process model transition sequence As we are dealing with event streams which need to be analyzed in a real-time manner, an event-granular replay strategy is required. Second, alternative approaches suffer from scalability issues which make them unsuitable in a real-time context.

A second remark entails the way decision points are resolved by the replayer. Put briefly, whenever multiple (enabled) transitions are mapped to the same event log activity within a process model and/or whenever multiple invisible activities are enabled, the replayer needs to determine which transition to execute to handle the activity at hand. Note that - in extreme edge cases - it is possible that the forced firing of a non-enabled transition should be preferred if this avoids several other violations later in the event trace [92]. A replay strategy is put forward which prefers the firing of enabled transition mapped to the activity at hand first, followed by the set of silent transitions, followed by the set of non-enabled transition mapped to the activity at hand. If the chosen set contains multiple transition candidates, a one-step look-ahead procedure is executed to determine which candidate enables the execution of the following activity (if no such candidate can be found, a random one is chosen). For the multitude of process models, this look-ahead suffices to resolve any ambiguities. However, since we are dealing with streaming event data in this context, we possess no knowledge about events which will arrive in the future, preventing the execution of the look-ahead procedure. There are three proposed strategies to deal with this issue. First, disabling the look-ahead altogether and assuming that the model is deterministic enough to handle incoming events without taking the context into account. Second (another extreme), restarting the replay of the full trace each time an event is added, thus allowing the replayer to revise earlier decisions. Note however that the replayer is configured such that no new violations may be introduced related to historical activities. In practice, this means that the replayer can revise the state chain by modifying the execution of silent transitions, selecting alternative albeit also enabled transition mapped to a particular activity for activities which were parsed correctly, or selecting alternative disabled transition, although only for activities which were not parsed correctly. The third method combines these two extremes by considering a part of the executed transition sequence as "frozen", only allowing revisions for the last $n$ steps.

As a third remark, as it is aforementioned, one of the drawbacks of "token game"-based replayers entails the possible creation of superfluous tokens, enabling subsequently for too much behavior. However, as was mentioned, the decomposition of a process model restricts the possible pernicious effects of the heuristics decisions taken during the conformance analysis, as each model is now limited to dealing with a smaller subset of behavior. In addition, as superfluous tokens are created following the forced firing of violating activities, the process instance or model fragment at hand is likely to be immediately indicated as "dubious" at this 
point, lowering the trustfulness of following events within this instance of model fragment, independent of the replay strategy being applied.

The results of the replay analysis can be reported and visualized. Remark that, naturally, these actions can be performed while the actual conformance analysis is running. In general, two ways of result follow-up are supported by our architecture. The first one consists of the logging of various statistics by the running worker threads and replayers, which is polled regularly by decoupled components (e.g. a real-time dashboard or perhaps logged to a persistent data store). The second manner by which results can be interpreted consists of the definitions of various triggers which are to be fired once certain criteria are met, such as a model fragment overshooting a certain error rate threshold, for instance, of a high-risk activity or model fragment being violated. The actions which can be undertaken as a result are self-explanatory, e.g. sending warnings, or halting running process instances or even the complete system.

\subsection{Case Example and Experimental Results}

In this section we propose the study of a realistic process case example in order to illustrate the approach presented its benefits. Model and logs - original and decomposed - of this case example, together with the rest of the benchmarks used in this experimental section, are publicly available ${ }^{1}$.

\subsubsection{Description}

The modeled process describes a realistic transaction process within a banking context. The process contains all sort of monetary checks, authority notifications, and logging mechanisms responding to the new degree of responsibility and accountability that current economic environments demand. The process is structured as follows (cf. Figure 9.3 shows a highlevel overview of the complete process): it is initiated when a new transaction is requested, opening a new instance in the system and registering all the components involved. The second step is to run a check on the person (or entity) origin of the monetary transaction. Then, the actual payment is processed differently, depending of the payment modality chosen by the sender (cash, cheque ${ }^{2}$ and payment). Later, the receiver is checked and the money is transferred. Finally, the process ends registering the information, notifying it to the required actors and authorities, and emitting the corresponding receipt.

The process has been modeled in terms of a Petri net. The decomposition techniques based on SESEs is used to decompose the overall model into suprocesses. In particular, a valid decomposition where components have size at most 60 is derived. Finally, the decomposition is post-processed by merging some of the SESEs in order to reach the final decomposition shown in Figure 9.4 (which depicts the full process): eight of the proposed subnets correspond

\footnotetext{
${ }^{1}$ doi:10.4121/uuid:c1d1fdbb-72df-470d-9315-d6f97e1d7c7c

${ }^{2}$ The British term is used to avoid ambiguity with the verb "to check".
} 


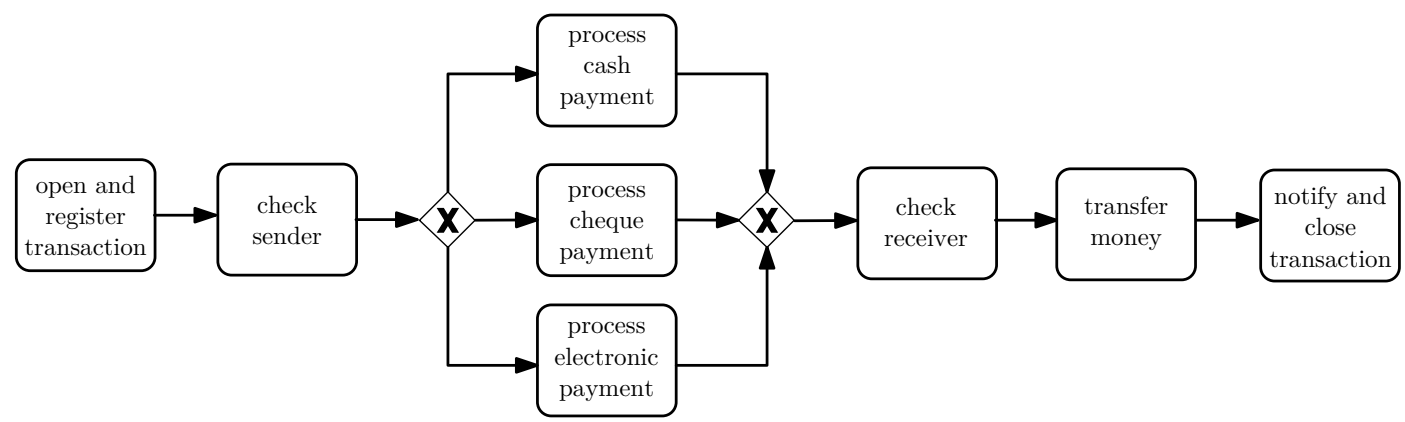

Figure 9.3: High level overview of the running example process, structured in subprocesses.

with the eight subprocesses identified in Figure 9.3 (represented within gray rectangles), and the ninth subnet contains all the trivial connections between suprocesses (represented outside the rectangles).

\subsubsection{Experimental Scenario Evaluation}

To illustrate the benefits of the technique, we present two possible scenarios within the case example process.

\section{Scenario 1: Serial Number Check}

The modeled process defines that, whenever a client executes the payment in cash, the serial numbers must be checked (see Figure 9.3). The banking regulation states that serial numbers must be compared with an external database governed by a recognized international authority ("Check Authority Serial Numbers CASN"). In addition, the bank of the case example decided to incorporate two complementary checks to its policy: an internal bank check ("Check Bank Serial Numbers CBSN"), and a check among the databases of the bank consortium this bank belongs to ("Check Inter-Bank Serial Numbers CIBSN"). At a given point, due to technical reasons (e.g., peak hour network congestion, malfunction of the software, deliberated blocking attack, etc.), the external check $C A S N$ is not longer performed, contradicting the modeled process, i.e., all the running instances of the process involving cash payment can proceed without the required check. Using the proposed approach, this situation is detected immediately, identifying the anomalous subprocess (process cash payment), focusing the conformance analysis on it, and eventually taking the necessary countermeasures. The consequences of detecting such cases only in forensic analysis performed months after the incident are severe and difficult to recover from. The situation is depicted in Figure 9.5. 

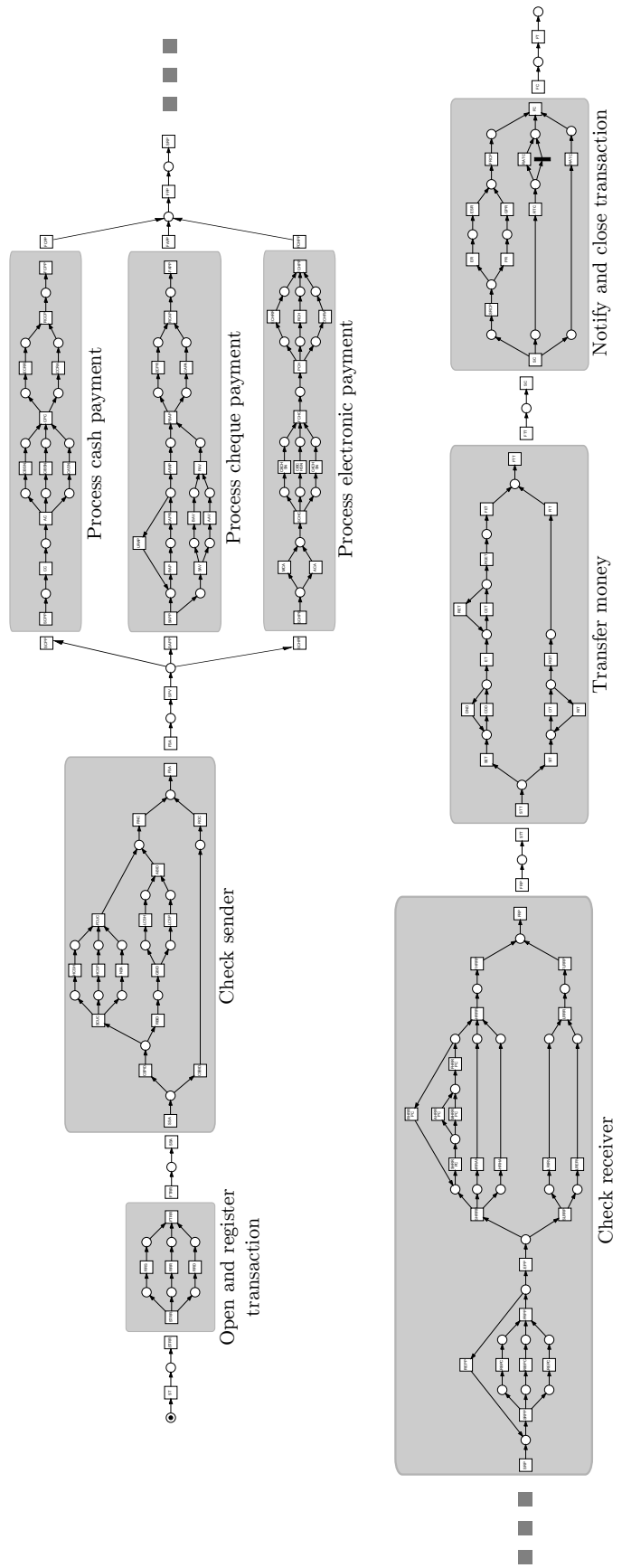

Figure 9.4: Running example: final valid SESE-decomposition.

The substructures are named according to Figure 9.3. 

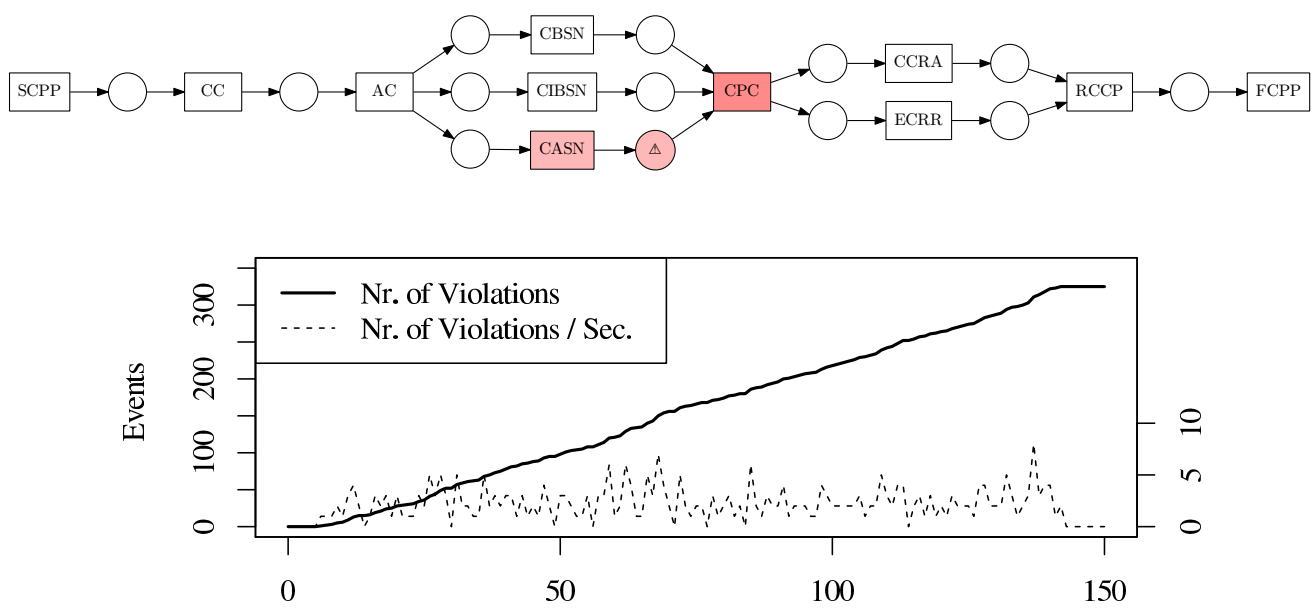

Time (Elapsed Seconds)

Figure 9.5: In the first scenario, the Check Authority Serial Number (CASN) activity is skipped for some process instances, causing the $C P C$ activity to fail, due to a missing input token which was expected to be present and placed there by the execution of $C A S N$. The figure depicts the error localized in the affected model fragment; the graph depicts the cumulative and running amount of violations detected within this fragment. 

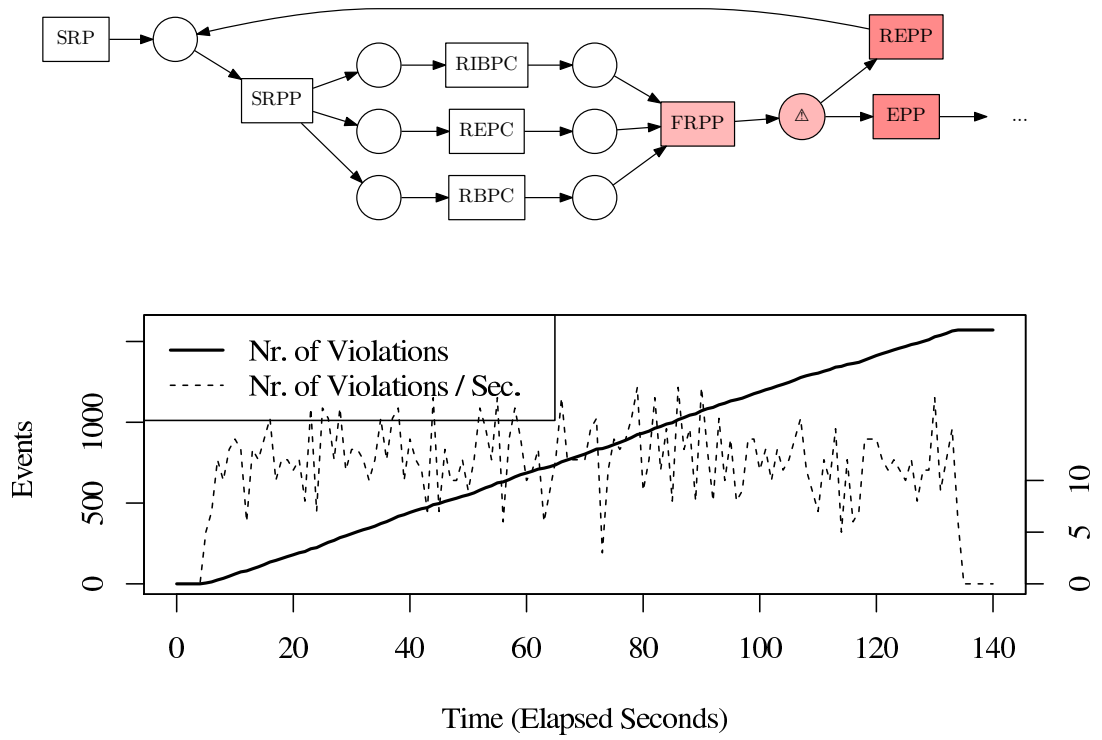

Figure 9.6: In the second scenario, the preliminary profile check for receivers is skipped (SRPP to FRPP), causing either the REPP or EPP activities to fail. The figure depicts the error localized in the affected model fragment; the graph depicts the cumulative and running amount of violations detected within this fragment.

\section{Scenario 2: Receiver Preliminary Profiling}

During the check receiver stage, the model establishes two steps to be performed sequentially: first, a preliminary profiling analysis ("Start Receiver Pre Profiling SRPP") is executed over the receiver in order to evaluate and establish its potential risk ("Evaluate Pre Profiling $E P P ")$. Only then, a complete background check is performed over the receiver, where this check can either be more casual ("Start Low Risk Receiver Processing SLRRP") or thoroughly ("Start High Risk Receiver Processing SHRRP") depending on the potential risk detected on the preliminary profiling. However, the presence of an inexperienced bank employee, malevolence, or simply a bad implemented bank evaluation protocol, could result in evaluating the receiver with an unfinished preliminary profile check. The situation is depicted in Figure 9.6. 


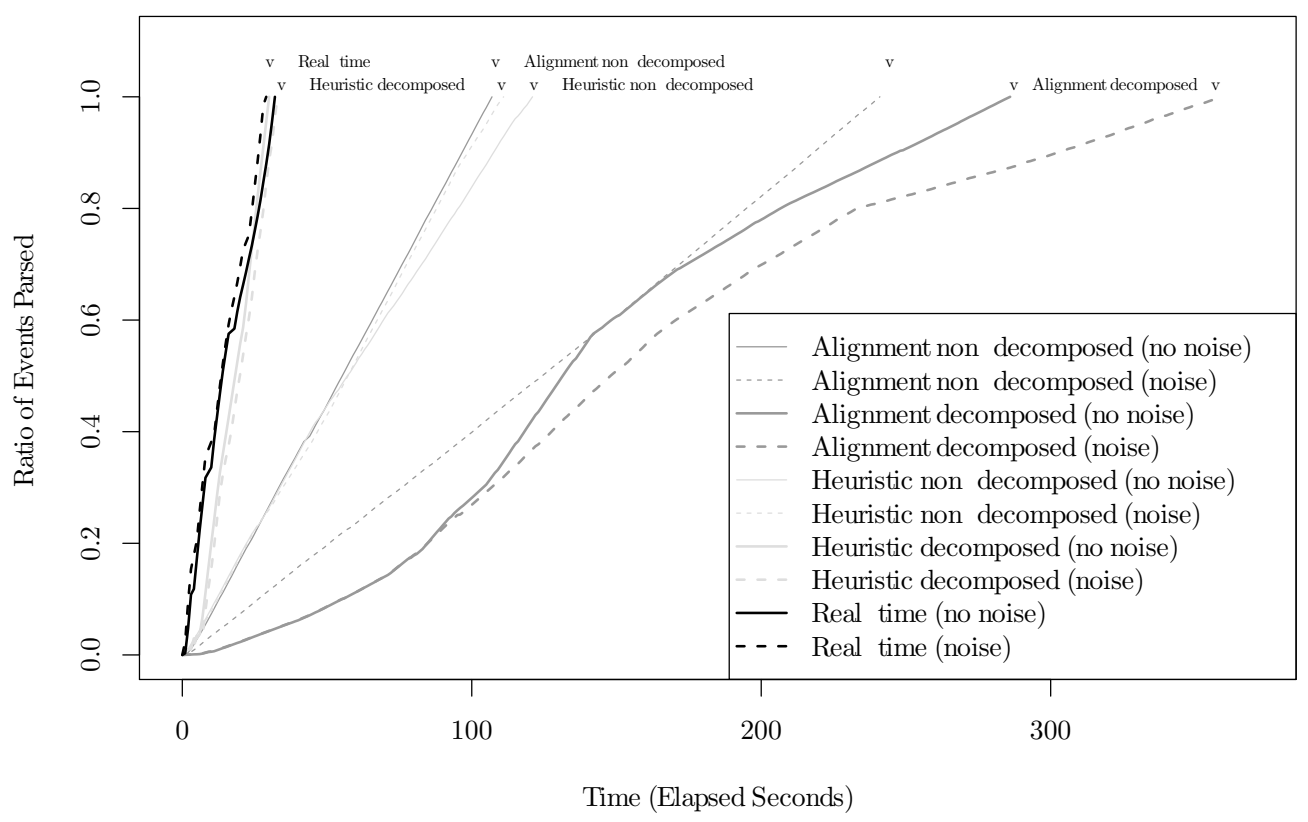

Figure 9.7: Comparison of replay performance for the included techniques in the experimental setup, showing the time taken per technique to replay the given event log.

\subsubsection{Experimental Comparison}

To benchmark the performance of our developed real-time conformance analysis technique against related approaches, a fitting event log was generated (based on the model depicted in Figure 9.4) containing ten thousand process instances (678864 events). A non-conforming ("noisy") variant of this event log was produced by inducing noise (inserting, deleting, and swapping of events) so that $10 \%$ of the included events are erroneous.

We compare our proposed technique against the alignment based replay technique by Adriansyah et al. [16] as well with the original implementation of the token-game based heuristic replayer [30]. Both the non-decomposed and decomposed variants of these techniques were included.

Figure 9.7 depicts the performance results of the experiment, showing the amount of time taken (x-axis) to check the conformance of the included event logs (the y-axis represents the cumulative ratio of event checks performed). As can be seen, our proposed real-time conformance analysis technique performs competitively with respect to related techniques. During the experimental run, a maximum throughput rate (number of events checked per 
second) was reached at 35000 with the experiment running on a single consumer laptop with three worker threads. Some additional remarks should be provided however when interpreting Figure 9.7. First, note that our proposed technique performs similarly as the heuristic decomposed replay technique, but note that proposed technique executes a conformance check on an event-granular basis and thus can be applied in a real-time monitoring setting, whereas the other techniques do so on a trace-granular level (i.e. a complete trace should be provided to perform the replay procedure). However, the event log is of sufficient size so that a step-wise effect is not apparent in Figure 9.7. Second, the replay procedure of the existing techniques was modified such that each trace is checked independently of the log context, meaning that no distinct trace grouping is performed over the log and each trace is checked as if it were belonging to an event log containing only this trace, so as to better assess the performance of these techniques in a real-time scenario (where the complete trace and $\log$ are unknown as events are arriving), rather than a post-hoc scenario where the complete event log is provided as-is. Note that - for the alignment based technique - this causes the non-decomposed version to perform better than the decomposed one. This is a perhaps unexpected result, but is caused by the fact that the alignment based techniques are geared towards checking - and as such expect - event logs as a whole. We thus emphasize the fact that these techniques have - currently — not been optimized to be applied in a real-time scenario (with an event stream being checked instead of an historical log).

\subsection{Conclusions}

In this chapter we have presented a novel business process conformance analysis technique which is able to support real-time monitoring of event-based data streams. Our approach offers a number of novel contributions, most notably a speed-up compared to related techniques, the ability to localize discrepancies and allowing real-time monitoring and thus rapid response times in mission-critical or high-risk environments, which is a significant benefit compared to existing conformance checking techniques which mainly work in an offline manner.

Future lines of research include: streamlining visualization and reporting capabilities, incorporating other decomposition and replay strategies, and adapting the framework into a distributed implementation, where different replayer engines run on separate machines. In addition, future research include the adaptation of these techniques to a strict steaming scenario, where the cases have no identification. 
Part IV

Closure 



\section{Chapter 10}

\section{Conclusions}

\subsection{Conclusion and Reflection}

In the early days of process mining, most of the research in process mining was mainly focused on discovery techniques, neglecting the importance of conformance. Several discovery approaches were presented, using a wide range of different techniques. However, measuring the adequacy of the provided results was never a priority, and only simple measures where used, being difficult to determine the most adequate approach for each case. Moreover, most analysis focused on verifying the properties of the model (e.g., has the model deadlocks?), or the properties of the $\log$ (e.g., is activity $\mathrm{x}$ and y always executed by a different person?), instead of evaluating whether the model represented faithfully the reality observed in the $\log$, i.e., conformance.

However, in the recent years this tendency has finally changed, and more research effort has been focused on the conformance dimensions. Since the thesis [79], several master and doctoral thesis has focused, totally or partially, on conformance. Thesis like [15], [26], [31], or the thesis presented here, represent a good example of that effort. In addition, several papers were presented in journals, conferences and workshops to analyze conformance: works addressing all conformance dimensions, such as [16, 1] for fitness, [62, 19, 30] for precision, $[30,8]$ for generalization, or [33] for simplicity, to enumerate some examples; works addressing the conformance using replay-based strategies such as [30], and also align-based strategies such as [16]; works addressing conformance in a decomposed way, such as [2, 1, 67]; and works addressing conformance for multi-perspective models such as [54]. New benchmarking frameworks for conformance analysis has been presented, such as [28], incorporating and using these new approaches, for example in [96], to evaluate the quality of state-of-the-art discovery algorithms. 


\subsection{Summary of Contributions}

The main theme of this thesis is the conformance checking and diagnosis between behavior observed in the log and process models. The contributions of the thesis can be divided in two groups according to the their purpose: techniques to evaluate the precision dimension, and techniques to decompose the conformance diagnosis.

- Precision in Conformance Checking. The precision is the dimension in conformance checking that measures the degree of a process model to describe only the behavior observed in the log, and no more.

Chapter 3 studied the limitations of the approaches based on comparing model and $\log$ relations, and presented a different approach to study precision. This approach is based on detecting escaping arcs, in other words, those points where the model allows more behavior than the one observed in the log. The escaping arcs are weighted and used to provide a metric for the precision dimension. Moreover the escaping arcs, and the situations leading to them, define possible points for a future actuation, in order to achieve a more precise model.

In Chapter 4, the escaping arcs theory is revisited to incorporate the notion of robustness. The refined approach consider the frequency of the observed traces in order to be less affected by infrequent or noisy observed behavior. The chapter also presented the notion of confidence over the precision metric provided. A narrow confidence interval indicates a high confidence on the provided metric, while a wide interval denotes a likely possibility that precision metric changes in the future. The confidence interval is determined according to a giving parameter indicating the future to consider, where a low value indicates a close future and a high value a distance future. The bounds of the interval represent the likelihood of new escaping arcs to appear, or disappear, within the considered future. Finally, the chapter presented a method to assess the severity of the escaping arcs detected. The severity proposed is based in a multi-factor analysis, with factors such as the weight of the escaping arc within the process or the criticality of the action allowed. Escaping arcs with high severity pinpoint imprecise situations that should be addressed urgently.

Chapter 5 presented a different approach to analyze the escaping arcs of a system. While the previous proposed technique detects the escaping arcs directly from the $\log$, this different approach uses a pre-processing step, where the observed behavior is aligned with the modeled behavior. Aligning observed and modeled behavior is able to solve in a global optimal way those situations, such as unfitting and undeterminism, where the direct use of the log would require the use of heuristics. However, the aligning of behaviors is time-consuming operation not suitable for all the scenarios. The chapter presented the use of aligned behavior to derive escaping arcs, including also those situations where the observed traces in the log can be optimally aligned with several model sequences. The chapter also proposed different levels of abstractions on 
the precision computation, where the order of the activities is not considered, or where the direction in which the log traces are processed is reverted to achieve a more balanced precision.

- Decomposed Conformance Diagnosis. Conformance checking is a time-consuming task. Moreover, identifying the causes of the conformance problems is sometimes far from easy. The decomposition techniques try to alleviate this two problems decomposing the processes in parts, and analyzing them separately. In this part, the conformance focus changes from the precision dimension of the previous part to the fitness dimension.

Chapter 6 studied the different decomposition methods in process mining, a proposed a decomposition conformance checking based on Single-Entry Single-Exit (SESE) components. SESEs are components with a clear interface with the rest of the model, (i.e., one entry node and one exit node), representing subprocesses within the main process. Decomposing a model in SESEs alleviate the conformance checking analysis, while at the same time, the meaningful decomposition provides a better understanding of what subprocesses have conformance problems.

Chapter 7 extended the diagnosis capacities of the decomposition methods, providing mechanisms to analyze the conformance in a topological way and a hierarchical way. A topology of a decomposition represents the connections between components, and it can be used to detect areas with conformance problems. A hierarchical conformance analysis allows to zoom-in and zoom-out on a hierarchy of components in order to get a better understanding of the situation and the cause of the conformance problems.

Chapter 8 proposes a decomposition of multi-perspective models, in order to alleviate the computation time and to aid in the conformance diagnosis. In particular, the decomposition proposed focus on models with control-flow and data perspective, aligning both flow and data in a distributed way.

Chapter 9 addresses the real-time monitoring of conformance deviations by means of decomposition and event-based heuristic replay. The decomposed setting aims at identifying the subprocesses cause of the conformance anomalies, while the replay adapts the analysis to the event-based nature of real-time monitoring scenarios.

\subsection{Challenges and Directions for Future Work}

The work presented in this thesis focus in the area of conformance checking, a first step to perform conformance analysis impossible until now. In this section we list some of the possible future research paths to follow:

- New metrics, new dimensions. Each conformance dimension states the property all metrics for that dimension should measure. However, the details on how this dimension is quantified relies on each specific metric, and each metric assumes a different 
interpretation of the dimension. For example, $P M[100]$ defines fitness as the number of correct parsed traces divided by the number of traces in the event log, while the metric $f[80]$ is more fine-grained because it also considers which problems (missing and remaining tokens) happened during the $\log$ replay. In precision, $a_{B}^{\prime}[80]$ derives sometimes follows and precedes relations for the model and the log and compares them, while etc $_{p}$ [62] bases its estimation on detecting escaping arcs. Each proposed metric has its advantages and disadvantages that make it appropriated for a particular scenario. For example, a precision metric such as $e t c_{p}$, less time consuming than $a_{B}^{\prime}$, is more suitable to be incorporated within a genetic algorithm where conformance is executed constantly [33]. Therefore, the study of new conformance metrics is an area that must be explored as future research, extending the base of metrics to use. Moreover, limiting conformance to only four dimensions (fitness, precision, generalization, and simplicity) may be considered too restrictive, and future research must be open to explore other possible dimensions, such as the completeness, i.e., is the model describing the complete process or only a part?

- Decomposed alignment of observed and modeled behavior. As experiments show, aligning observed and modeled behavior can be a time-consuming tasks [15]. Chapter 6 and other similar works alleviate this cost by means of decomposing the process. However, as it is remarked in the same chapter, the goal of decomposing is not to aligning the whole log and model (i.e., the original problem), but to align at component level to provide localized conformance information. It is proven by counterexample that the simple concatenation of local optimal alignments does not result in a global optimal alignment. However, the use of decomposed techniques have provided equally important results for the global setting. For example, [1] provides a lower bound on the global alignment cost based on local alignments. The use of decomposed techniques is a promising research path to alleviate the cost of the alignments, and lots of efforts has been put in the recent months. That may include, for example, the study of what properties the traces, model, or local alignments must satisfy so the lower bound results in the exact cost, or the development of divide-and-conquer strategies for a bottom-up construction of the alignments.

- Decomposed conformance for other dimensions. Chapter 6 shows how to check perfectly fitting traces in a decomposed way. However, conformance checking is composed by other three dimensions. The use of decomposed techniques to measure those dimensions is an interesting future research path, but it is also a challenge far from trivial. For example, metrics such as etc $c_{p}[62]$ and $a_{B}^{\prime}$ [80] define precision as a global property, and therefore, a direct decomposition is not possible. Several are the options to explore to address that issue: the real cost of metrics like $a_{p}[19]$ comes from computing the alignments they rely on, and thus, a faster decomposed alignment of the behaviors will reduce the computation cost of the metric; in other cases, a simple conformance analysis can be done at local level to then be smartly aggregated at a 
global level, similar to a Map Reduce strategy; a final option is the definition of new metrics that consider a redefinition of the dimension in a non-global way. Similarly, decomposition can be used to measure simplicity, for example, measuring the simplicity of the components and how they are connected.

- Visualization and diagnosis. The usefulness of results is directly related with how the results are provided. Results poorly displayed may limit their transmitted information. This is specially important in the conformance checking area, where results tend to be large (e.g., set of escaping arcs, tokens missing, misalignments, ...) and pinpointing future lines of actuation withing the organization. The thesis provides several mechanisms in that direction (e.g., severity of the escaping arcs, or detection of problematic areas using the topology of a decomposition). However, this is just the tip of the iceberg and more approach should follow. Some future work possibilities include the aggregation of conformance problems by the underlying cause behind them, or new ways of visually represent those problems.

- Model repair. The conformance diagnosis approaches presented in this thesis indicate points where the model does not describe adequately the reality. These points indicate possible parts of the model to be repaired, but the approaches rely on the user to perform iterative improvements on the model. The use of the conformance results within a fully automated approach to repair the models, similar to [44] and [35], is an interesting topic for further research. This becomes specially interesting in the decomposed setting, where the model can be repaired component by component. Moreover, other scenarios to consider are also possible, such as models that cannot be modified, opening the door to theories like supervisory control [76] in order to derive a controller to supervise the model execution. 



\section{Bibliography}

[1] Wil M. P. van der Aalst. "Decomposing Petri nets for process mining: A generic approach". In: Distributed and Parallel Databases 31.4 (2013), pp. 471-507 (cit. on pp. 27, 41, 117, 119, 121, 123-125, 128, 132, 158, 179, 182).

[2] Wil M. P. van der Aalst. "Decomposing Process Mining Problems Using Passages". In: 33rd International Conference on Application and Theory of Petri Nets, PETRI NETS '12. Ed. by Serge Haddad and Lucia Pomello. Vol. 7347. Lecture Notes in Computer Science. Springer, 2012, pp. 72-91 (cit. on pp. 116, 117, 131, 139, 179).

[3] Wil M. P. van der Aalst. "Formalization and verification of event-driven process chains". In: Information \& Software Technology 41.10 (1999), pp. 639-650 (cit. on p. 36).

[4] Wil M. P. van der Aalst. Process Mining - Discovery, Conformance and Enhancement of Business Processes. Springer, 2011, pp. I-XVI, 1-352 (cit. on pp. 17, 23, 41, 115).

[5] Wil M. P. van der Aalst. "The Application of Petri Nets to Workflow Management". In: Journal of Circuits, Systems, and Computers 8.1 (1998), pp. 21-66 (cit. on p. 36).

[6] Wil M. P. van der Aalst. "Using Process Mining to Generate Accurate and Interactive Business Process Maps". In: Business Information Systems Workshops, BIS'09. Ed. by Witold Abramowicz and Dominik Flejter. Vol. 37. Lecture Notes in Business Information Processing. Springer, 2009, pp. 1-14 (cit. on p. 139).

[7] Wil M. P. van der Aalst. "Verification of Workflow Nets". In: Application and Theory of Petri Nets, PETRI NETS '9\%. Ed. by Pierre Azéma and Gianfranco Balbo. Vol. 1248. Lecture Notes in Computer Science. Springer, 1997, pp. 407-426 (cit. on p. 36).

[8] Wil M. P. van der Aalst, Arya Adriansyah, and Boudewijn F. van Dongen. "Replaying history on process models for conformance checking and performance analysis". In: Wiley Interdisc. Rew.: Data Mining and Knowledge Discovery 2.2 (2012), pp. 182-192 (cit. on pp. 41, 179).

[9] Wil M. P. van der Aalst and Arthur H. M. ter Hofstede. "YAWL: yet another workflow language". In: Inf. Syst. 30.4 (2005), pp. 245-275 (cit. on pp. 18, 36, 37). 
[10] Wil M. P. van der Aalst, Arthur H. M. ter Hofstede, Bartek Kiepuszewski, and Alistair P. Barros. "Workflow Patterns". In: Distributed and Parallel Databases 14.1 (2003), pp. 5-51 (cit. on p. 37).

[11] Wil M. P. van der Aalst, Hajo A. Reijers, and Minseok Song. "Discovering Social Networks from Event Logs". In: Computer Supported Cooperative Work 14.6 (2005), pp. 549-593 (cit. on p. 18).

[12] Wil M. P. van der Aalst, Vladimir Rubin, H. M. W. Verbeek, Boudewijn F. van Dongen, Ekkart Kindler, and Christian W. Günther. "Process mining: a two-step approach to balance between underfitting and overfitting". In: Software and System Modeling 9.1 (2010), pp. 87-111 (cit. on pp. 49, 101, 102).

[13] Wil M. P. van der Aalst, Ton Weijters, and Laura Maruster. "Workflow Mining: Discovering Process Models from Event Logs". In: IEEE Trans. Knowl. Data Eng. 16.9 (2004), pp. 1128-1142 (cit. on pp. 18, 83).

[14] Rafael Accorsi and Thomas Stocker. "On the exploitation of process mining for security audits: the conformance checking case". In: Proceedings of the ACM Symposium on Applied Computing, SAC 2012, Riva, Trento, Italy, March 26-30, 2012. Ed. by Sascha Ossowski and Paola Lecca. ACM, 2012, pp. 1709-1716 (cit. on p. 43).

[15] Arya Adriansyah. "Aligning Observed and Modeled Behavior". PhD thesis. Eindhoven, The Netherlands: Technische Universiteit Eindhoven, 2014 (cit. on pp. 18, 21, 27, 61, 78, 87-91, 116, 179, 182).

[16] Arya Adriansyah, Boudewijn F. van Dongen, and Wil M. P. van der Aalst. "Conformance Checking Using Cost-Based Fitness Analysis". In: 15th IEEE International Enterprise Distributed Object Computing Conference, EDOC'11. IEEE Computer Society, 2011, pp. 55-64 (cit. on pp. 41, 78, 90, 91, 115, 132, 149, 151, 169, 175, 179).

[17] Arya Adriansyah, Boudewijn F. van Dongen, and Wil M. P. van der Aalst. MemoryEfficient Alignment of Observed and Modeled Behavior. Tech. rep. BPM-03-03. BPMcenter.org, 2013 (cit. on pp. 90, 91).

[18] Arya Adriansyah, Jorge Munoz-Gama, Josep Carmona, Boudewijn F. van Dongen, and Wil M. P. van der Aalst. "Alignment Based Precision Checking". In: 8th International Workshop on Business Process Intelligence, BPI'12. Ed. by Marcello La Rosa and Pnina Soffer. Vol. 132. Lecture Notes in Business Information Processing. Springer, 2012, pp. 137-149 (cit. on pp. 24, 26, 62, 85, 98, 195).

[19] Arya Adriansyah, Jorge Munoz-Gama, Josep Carmona, Boudewijn F. van Dongen, and Wil M. P. van der Aalst. "Measuring precision of modeled behavior". In: Inf. Syst. E-Business Management 12 (2014). (to appear) (cit. on pp. 24, 26, 41, 62, 85, $179,182,196)$. 
[20] Arya Adriansyah, Natalia Sidorova, and Boudewijn F. van Dongen. "Cost-Based Fitness in Conformance Checking". In: 11th International Conference on Application of Concurrency to System Design, ACSD'11. Ed. by Benoît Caillaud, Josep Carmona, and Kunihiko Hiraishi. IEEE, 2011, pp. 57-66 (cit. on pp. 41, 90, 91).

[21] Sebastian Banescu, Milan Petkovic, and Nicola Zannone. "Measuring Privacy Compliance Using Fitness Metrics". In: 10th International Conference on Business Process Management, BPM'12. Ed. by Alistair P. Barros, Avigdor Gal, and Ekkart Kindler. Vol. 7481. Lecture Notes in Computer Science. Springer, 2012, pp. 114-119 (cit. on p. 41).

[22] Fábio de Lima Bezerra and Jacques Wainer. "Algorithms for anomaly detection of traces in logs of process aware information systems". In: Inf. Syst. 38.1 (2013), pp. 3344 (cit. on p. 67).

[23] Fábio de Lima Bezerra, Jacques Wainer, and Wil M. P. van der Aalst. "Anomaly Detection Using Process Mining". In: 10th International Workshop on Business Process Modeling, Development, and Support, BPMDS'09. Ed. by Terry A. Halpin et al. Vol. 29. Lecture Notes in Business Information Processing. Springer, 2009, pp. 149 161 (cit. on p. 67).

[24] R. P. Jagadeesh Chandra Bose, Wil M. P. van der Aalst, Indre Zliobaite, and Mykola Pechenizkiy. "Handling Concept Drift in Process Mining". In: Advanced Information Systems Engineering, CAiSE '11. Ed. by Haralambos Mouratidis and Colette Rolland. Vol. 6741. Lecture Notes in Computer Science. Springer, 2011, pp. 391-405 (cit. on p. 126).

[25] George E. P. Box, William G. Hunter, and J. Stuart Hunter. Statistics for experimenters : an introduction to design, data analysis, and model building. Wiley series in probability and mathematical statistics. J. Wiley \& Sons, 1978 (cit. on p. 77).

[26] Seppe K. L. M. vanden Broucke. "Advances in Process Mining: Artificial Negative Events and Other Techniques". PhD thesis. Leuven, Belgium: Katholieke Universiteit Leuven, 2014 (cit. on pp. 168, 179).

[27] Seppe K. L. M. vanden Broucke, Jochen De Weerdt, Bart Baesens, and Jan Vanthienen. "Improved Artificial Negative Event Generation to Enhance Process Event Logs". In: 24th International Conference on Advanced Information Systems Engineering, CAiSE'12. Ed. by Jolita Ralyté, Xavier Franch, Sjaak Brinkkemper, and Stanislaw Wrycza. Vol. 7328. Lecture Notes in Computer Science. Springer, 2012, pp. 254-269 (cit. on pp. 43, 57, 106).

[28] Seppe K. L. M. vanden Broucke, Jochen De Weerdt, Jan Vanthienen, and Bart Baesens. "A comprehensive benchmarking framework (CoBeFra) for conformance analysis between procedural process models and event logs in ProM". In: IEEE Symposium on Computational Intelligence and Data Mining, CIDM'13. IEEE, 2013, pp. 254-261 (cit. on pp. 26, 106, 179). 
[29] Seppe K.L.M. vanden Broucke, Jorge Munoz-Gama, Josep Carmona, Bart Baesens, and Jan Vanthienen. "Event-based Real-time Decomposed Conformance Analysis". In: International Conference on Cooperative Information Systems, CoopIS'14. Ed. by Robert Meersman et al. Springer, 2014, pp. 345-363 (cit. on pp. 24, 26, 165, 196).

[30] Seppe K.L.M. vanden Broucke, Jochen De Weerdt, Jan Vanthienen, and Bart Baesens. "Determining Process Model Precision and Generalization with Weighted Artificial Negative Events". In: IEEE Transactions on Knowledge and Data Engineering 99.PrePrints (2013), p. 1 (cit. on pp. 86, 145, 168, 169, 175, 179).

[31] Joos C. A. M. Buijs. "Flexible Evolutionary Algorithms for Mining Structured Process Models". PhD thesis. Eindhoven, The Netherlands: Technische Universiteit Eindhoven, 2014 (cit. on p. 179).

[32] Joos C. A. M. Buijs, Boudewijn F. van Dongen, and Wil M. P. van der Aalst. "A genetic algorithm for discovering process trees". In: IEEE Congress on Evolutionary Computation. IEEE, 2012, pp. 1-8 (cit. on p. 55).

[33] Joos C. A. M. Buijs, Boudewijn F. van Dongen, and Wil M. P. van der Aalst. "On the Role of Fitness, Precision, Generalization and Simplicity in Process Discovery". In: On the Move to Meaningful Internet Systems, OTM'12. Ed. by Robert Meersman et al. Vol. 7565. Lecture Notes in Computer Science. Springer, 2012, pp. 305-322 (cit. on pp. $22,26,41,42,179,182)$.

[34] Joos C. A. M. Buijs, Boudewijn F. van Dongen, and Wil M. P. van der Aalst. "Towards Cross-Organizational Process Mining in Collections of Process Models and Their Executions". In: Business Process Management Workshops - BPM 2011 International Workshops, Clermont-Ferrand, France, August 29, 2011, Revised Selected Papers, Part II. Ed. by Florian Daniel, Kamel Barkaoui, and Schahram Dustdar. Springer, 2011, pp. 2-13 (cit. on p. 109).

[35] Joos C. A. M. Buijs, Marcello La Rosa, Hajo A. Reijers, Boudewijn F. van Dongen, and Wil M. P. van der Aalst. "Improving Business Process Models Using Observed Behavior". In: Data-Driven Process Discovery and Analysis, SIMPDA'12. Ed. by Philippe Cudré-Mauroux, Paolo Ceravolo, and Dragan Gasevic. Vol. 162. Lecture Notes in Business Information Processing. Springer, 2012, pp. 44-59 (cit. on p. 183).

[36] Andrea Burattin and Alessandro Sperduti. "PLG: A Framework for the Generation of Business Process Models and Their Execution Logs". In: Business Process Management Workshops - BPM 2010 International Workshops and Education Track, Hoboken, NJ, USA, September 13-15, 2010, Revised Selected Papers. Ed. by Michael zur Muehlen and Jianwen Su. Springer, 2010, pp. 214-219 (cit. on p. 82).

[37] Josep Carmona, Jordi Cortadella, and Michael Kishinevsky. "Genet: A Tool for the Synthesis and Mining of Petri Nets". In: Application of Concurrency to System Design, $A C S D$ '09. IEEE Computer Society, 2009, pp. 181-185 (cit. on pp. 18, 83). 
[38] Thomas H. Cormen, Charles E. Leiserson, Ronald L. Rivest, and Clifford Stein. Introduction to Algorithms (3. ed.) MIT Press, 2009, pp. I-XIX, 1-1292 (cit. on p. 73).

[39] Boudewijn F. van Dongen, Remco M. Dijkman, and Jan Mendling. "Measuring Similarity between Business Process Models". In: 20th International Conference on Advanced Information Systems Engineering, CAiSE'08. Ed. by Zohra Bellahsene and Michel Léonard. Vol. 5074. Lecture Notes in Computer Science. Springer, 2008, pp. 450464 (cit. on p. 43).

[40] Boudewijn F. van Dongen, Jan Mendling, and Wil M. P. van der Aalst. "Structural Patterns for Soundness of Business Process Models". In: 10th IEEE International Enterprise Distributed Object Computing Conference, EDOC'06. IEEE Computer Society, 2006, pp. 116-128 (cit. on p. 43).

[41] Marlon Dumas, Wil M. P. van der Aalst, and Arthur H. M. ter Hofstede. ProcessAware Information Systems: Bridging People and Software Through Process Technology. Wiley, 2005 (cit. on pp. 41, 42).

[42] Marlon Dumas, Luciano García-Bañuelos, and Artem Polyvyanyy. "Unraveling Unstructured Process Models". In: Second International Workshop on Business Process Modeling Notation, BPMN'10. Ed. by Jan Mendling, Matthias Weidlich, and Mathias Weske. Springer, 2010, pp. 1-7 (cit. on p. 160).

[43] Extensible Event Stream (XES). www.xes-standard.org (cit. on p. 30).

[44] Dirk Fahland and Wil M. P. van der Aalst. "Repairing Process Models to Reflect Reality". In: Business Process Management, BPM'12. Ed. by Alistair P. Barros, Avigdor Gal, and Ekkart Kindler. Vol. 7481. Lecture Notes in Computer Science. Springer, 2012, pp. 229-245 (cit. on pp. 18, 183).

[45] Dirk Fahland, Massimiliano de Leoni, Boudewijn F. van Dongen, and Wil M. P. van der Aalst. "Conformance Checking of Interacting Processes with Overlapping Instances". In: Business Process Management, BPM'11. Ed. by Stefanie Rinderle-Ma, Farouk Toumani, and Karsten Wolf. Vol. 6896. Lecture Notes in Computer Science. Springer, 2011 (cit. on p. 117).

[46] Stijn Goedertier, David Martens, Jan Vanthienen, and Bart Baesens. "Robust Process Discovery with Artificial Negative Events". In: Journal of Machine Learning Research 10 (2009), pp. 1305-1340 (cit. on pp. 41, 43, 57).

[47] Gianluigi Greco, Antonella Guzzo, Luigi Pontieri, and Domenico Saccà. "Discovering Expressive Process Models by Clustering Log Traces". In: IEEE Trans. Knowl. Data Eng. 18.8 (2006), pp. 1010-1027 (cit. on pp. 41, 43).

[48] John E. Hopcroft and Robert Endre Tarjan. "Dividing a Graph into Triconnected Components". In: SIAM J. Comput. 2.3 (1973), pp. 135-158 (cit. on p. 117).

[49] IEEE Task Force on Process Mining - Case Studies. http://www.win.tue .nl/ ieeetfpm/doku.php?id=shared:process_mining_case_studies (cit. on p. 115). 
[50] K. Jensen and L.M. Kristensen. Coloured Petri Nets. Springer Verlag, 2009 (cit. on p. 152).

[51] Brian Karrer, Elizaveta Levina, and M. E. J. Newman. "Robustness of community structure in networks". In: Phys. Rev. E 77.4 (Apr. 2008), p. 046119 (cit. on p. 77).

[52] George Karypis and Vipin Kumar. "A fast and high quality multilevel scheme for partitioning irregular graphs". In: 1998, pp. 359-392 (cit. on p. 116).

[53] Sander J. J. Leemans, Dirk Fahland, and Wil M. P. van der Aalst. "Discovering BlockStructured Process Models from Event Logs - A Constructive Approach". In: 34th International Conference on Application and Theory of Petri Nets and Concurrency, PETRI NETS 2013. Ed. by José Manuel Colom and Jörg Desel. Springer, 2013, pp. 311-329 (cit. on p. 131).

[54] Massimiliano de Leoni and Wil M. P. van der Aalst. "Aligning Event Logs and Process Models for Multi-perspective Conformance Checking: An Approach Based on Integer Linear Programming". In: 11th International Conference on Business Process Management, BPM '13. Ed. by Florian Daniel, Jianmin Wang, and Barbara Weber. Vol. 8094. Lecture Notes in Computer Science. Springer, 2013, pp. 113-129 (cit. on pp. 21, 23, 41, 151, 152, 161, 162, 179).

[55] Massimiliano de Leoni, Jorge Munoz-Gama, Josep Carmona, and Wil M.P. van der Aalst. "Decomposing Alignment-based Conformance Checking of Data-aware Process Models". In: International Conference on Cooperative Information Systems, CoopIS'14. Ed. by Robert Meersman et al. Springer, 2014, pp. 3-20 (cit. on pp. 24, 26, 149, 196).

[56] Peter Liggesmeyer. Software-Qualität - testen, analysieren und verifizieren von Software. Spektrum Akadem. Verl., 2002, pp. I-XV, 1-523 (cit. on p. 54).

[57] F. Mannhardt, M. de Leoni, and H.A. van der Aalst Wil M. P. Reijers. Balanced Multi-Perspective Checking of Process Conformance. Tech. rep. BPM Center Report BPM-14-07. BPMcenter.org, 2014 (cit. on pp. 151, 162).

[58] Ana Karla A. de Medeiros. "Genetic Process Mining". PhD thesis. Eindhoven, The Netherlands: Technische Universiteit Eindhoven, 2006 (cit. on pp. 18, 43).

[59] Ana Karla A. de Medeiros, Wil M. P. van der Aalst, and A. J. M. M. Weijters. "Quantifying process equivalence based on observed behavior". In: Data Knowl. Eng. 64.1 (2008), pp. 55-74 (cit. on pp. 41, 43).

[60] Ana Karla A. de Medeiros, A. J. M. M. Weijters, and Wil M. P. van der Aalst. "Genetic process mining: an experimental evaluation". In: Data Min. Knowl. Discov. 14.2 (2007), pp. 245-304 (cit. on pp. 41, 43, 55, 65).

[61] Jorge Munoz-Gama. "Algorithms for Process Conformance and Process Refinement". MA thesis. Barcelona, Spain: Universitat Politecnica de Catalunya, 2010 (cit. on p. 57). 
[62] Jorge Munoz-Gama and Josep Carmona. "A Fresh Look at Precision in Process Conformance". In: 8th International Conference on Business Process Management, BPM'10. Ed. by Richard Hull, Jan Mendling, and Stefan Tai. Vol. 6336. Lecture Notes in Computer Science. Springer, 2010, pp. 211-226 (cit. on pp. 18, 24, 26, 41, 102, 106, 179, 182, 195).

[63] Jorge Munoz-Gama and Josep Carmona. "A General Framework for Precision Checking". In: Special Issue on Intelligent and Innovative Computing in Business Process Management of International Journal of Innovative Computing, Information and Control 8.7(B) (July 2012), pp. 5317-5339 (cit. on pp. 24, 26, 65, 102, 195).

[64] Jorge Munoz-Gama and Josep Carmona. "Enhancing precision in Process Conformance: Stability, confidence and severity". In: IEEE Symposium on Computational Intelligence and Data Mining, CIDM'11. IEEE, 2011, pp. 184-191 (cit. on pp. 24, 26, $65,102,195)$.

[65] Jorge Munoz-Gama, Josep Carmona, and Wil M. P. van der Aalst. "Conformance Checking in the Large: Partitioning and Topology". In: 11th International Conference on Business Process Management, BPM'13. Ed. by Florian Daniel, Jianmin Wang, and Barbara Weber. Vol. 8094. Lecture Notes in Computer Science. [Best Student Paper Award]. Springer, 2013, pp. 130-145 (cit. on pp. 24, 26, 115, 119, 131, 132, 135, 139, 196).

[66] Jorge Munoz-Gama, Josep Carmona, and Wil M. P. van der Aalst. "Hierarchical Conformance Checking of Process Models Based on Event Logs". In: 34th International Conference on Application and Theory of Petri Nets and Concurrency, PETRI NETS'13. Ed. by José Manuel Colom and Jörg Desel. Vol. 7927. Lecture Notes in Computer Science. Springer, 2013, pp. 291-310 (cit. on pp. 24, 26, 119, 131, 132, 139, $144,145,148,160,196)$.

[67] Jorge Munoz-Gama, Josep Carmona, and Wil M.P. van der Aalst. "Single-Entry Single-Exit Decomposed Conformance Checking". In: Information Systems 46 (2014), pp. 102-122 (cit. on pp. 18, 24, 26, 27, 115, 119, 139, 179, 196).

[68] Tadao Murata. "Petri nets: Properties, analysis and applications". In: Proceedings of the IEEE 77.4 (1989), pp. 541-580 (cit. on pp. 17, 23, 34).

[69] OMG. Business Process Model and Notation (BPMN) Version 2.0. http://www .omg. org/spec/BPMN/2.0/. 2011 (cit. on pp. 17, 36, 37).

[70] OMG. Unified Modeling Language (UML) Version 2.0. www.omg.org/spec/UML/2.0/. 2011 (cit. on pp. 36, 37).

[71] Milan Petkovic, Davide Prandi, and Nicola Zannone. "Purpose Control: Did You Process the Data for the Intended Purpose?" In: 8th VLDB Workshop on Secure Data Management, SDM'11. Ed. by Willem Jonker and Milan Petkovic. Vol. 6933. Lecture Notes in Computer Science. Springer, 2011, pp. 145-168 (cit. on p. 41). 
[72] Artem Polyvyanyy. "Structuring process models". PhD thesis. University of Potsdam, 2012 (cit. on pp. 116, 117, 160).

[73] Artem Polyvyanyy, Jussi Vanhatalo, and Hagen Völzer. "Simplified Computation and Generalization of the Refined Process Structure Tree". In: 7th International Workshop on Web Services and Formal Methods, WSFM'10. Ed. by Mario Bravetti and Tevfik Bultan. Vol. 6551. Lecture Notes in Computer Science. Springer, 2010, pp. 25-41 (cit. on pp. 116-119).

[74] Process Mining. http://www.processmining.org (cit. on p. 57).

[75] ProM 6 Framework. http://www.promtools.org/prom6 (cit. on p. 57).

[76] Peter J Ramadge and W Murray Wonham. "Supervisory control of a class of discrete event processes". In: SIAM Journal on Control and Optimization 25.1 (1987), pp. 206230 (cit. on pp. 57, 183)

[77] Wolfgang Reisig and Grzegorz Rozenberg. Lectures on Petri nets I: Basic models Advances in Petri nets. Vol. 1491. Springer, 1998 (cit. on p. 45).

[78] Joel Ribeiro, Josep Carmona, Mustafa Misir, and Michele Sebag. "A Recommender System for Process Discovery". In: Business Process Management, BPM'14. Lecture Notes in Computer Science. Springer, 2014 (cit. on p. 26).

[79] Anne Rozinat. "Process Mining: Conformance and Extension". PhD thesis. Eindhoven, The Netherlands: Technische Universiteit Eindhoven, 2010 (cit. on pp. 18, 19, $22,23,27,43-45,96,179)$.

[80] Anne Rozinat and Wil M. P. van der Aalst. "Conformance checking of processes based on monitoring real behavior". In: Inf. Syst. 33.1 (2008), pp. 64-95 (cit. on pp. 19, 21, $22,25,41-43,45,52,57,58,62,80,88,96,115,145,149,168,182)$.

[81] Anne Rozinat and Wil M. P. van der Aalst. Conformance Testing: Measuring the Alignment Between Event Logs and Process Models. BETA Research School for Operations Management and Logistics, 2005 (cit. on p. 54).

[82] Anne Rozinat and Wil M. P. van der Aalst. "Conformance Testing: Measuring the Fit and Appropriateness of Event Logs and Process Models". In: Business Process Management Workshops. Ed. by Christoph Bussler and Armin Haller. Vol. 3812. 2005, pp. 163-176 (cit. on p. 22).

[83] Anne Rozinat and Wil M. P. van der Aalst. "Decision Mining in ProM". In: Business Process Management, BPM'06. Ed. by Schahram Dustdar, José Luiz Fiadeiro, and Amit P. Sheth. Vol. 4102. Lecture Notes in Computer Science. Springer, 2006, pp. 420 425 (cit. on p. 19). 
[84] Anne Rozinat, Ana Karla Alves de Medeiros, Christian W. Günther, A. J. M. M. Weijters, and Wil M. P. van der Aalst. "The Need for a Process Mining Evaluation Framework in Research and Practice". In: Business Process Management Workshops. Ed. by Arthur H. M. ter Hofstede, Boualem Benatallah, and Hye-Young Paik. Vol. 4928. Lecture Notes in Computer Science. Springer, 2007, pp. 84-89 (cit. on p. 22).

[85] Anne Rozinat, Manuela Veloso, and Wil M. P. van der Aalst. Using Hidden markov models to evaluate the quality of discovered process models. Tech. rep. BPM-08-10. BPMcenter.org, 2008 (cit. on p. 41).

[86] P. Sarbanes, G. Oxley, et al. Sarbanes-Oxley Act of 2012. 2002 (cit. on p. 42).

[87] Alexander Schrijver. Theory of linear and integer programming. Wiley-Interscience series in discrete mathematics and optimization. Wiley, 1999, pp. I-XI, 1-471 (cit. on p. 73$)$.

[88] Manuel Silva, Enrique Teruel, and José Manuel Colom. "Linear Algebraic and Linear Programming Techniques for the Analysis of Place or Transition Net Systems". In: Petri Nets. Ed. by Wolfgang Reisig and Grzegorz Rozenberg. Vol. 1491. Lecture Notes in Computer Science. Springer, 1996, pp. 309-373 (cit. on p. 145).

[89] Sergey Smirnov, Matthias Weidlich, and Jan Mendling. "Business Process Model Abstraction Based on Behavioral Profiles". In: 8th International Conference on ServiceOriented Computing, ICSOC. Ed. by Paul P. Maglio, Mathias Weske, Jian Yang, and Marcelo Fantinato. Vol. 6470. Lecture Notes in Computer Science. 2010, pp. 1-16 (cit. on pp. 168, 169).

[90] Marc Solé and Josep Carmona. "Rbminer: A Tool for Discovering Petri Nets from Transition Systems". In: Automated Technology for Verification and Analysis, ATVA'10. Ed. by Ahmed Bouajjani and Wei-Ngan Chin. Vol. 6252. Lecture Notes in Computer Science. Springer, 2010, pp. 396-402 (cit. on pp. 58, 83).

[91] Robert Endre Tarjan and Jacobo Valdes. "Prime Subprogram Parsing of a Program". In: Principles of Programming Languages, POPL'80. Ed. by Paul W. Abrahams, Richard J. Lipton, and Stephen R. Bourne. ACM Press, 1980, pp. 95-105 (cit. on p. 116).

[92] S. vanden Broucke, J. De Weerdt, J. Vanthienen, and B. Baesens. On replaying process execution traces containing positive and negative events. FEB Research Report KBI 1311. KU Leuven, 2013, 17 pp. (Cit. on p. 169).

[93] Jussi Vanhatalo, Hagen Völzer, and Jana Koehler. "The Refined Process Structure Tree". In: Business Process Management, BPM'08. Ed. by Marlon Dumas, Manfred Reichert, and Ming-Chien Shan. Vol. 5240. Lecture Notes in Computer Science. Springer, 2008, pp. 100-115 (cit. on p. 116).

[94] Jussi Vanhatalo, Hagen Völzer, and Jana Koehler. "The refined process structure tree". In: Data Knowl. Eng. 68.9 (2009), pp. 793-818 (cit. on pp. 116, 119). 
[95] J. de Weerdt, M. de Backer, J. Vanthienen, and B. Baesens. "A Critical Evaluation Study of Model-log Metrics in Process Discovery". In: Business Process Management Workshops. Ed. by Michael Muehlen and Jianwen Su. Vol. 66. Lecture Notes in Business Information Processing. Springer Berlin Heidelberg, 2011, pp. 158-169 (cit. on p. 106).

[96] Jochen De Weerdt, Manu De Backer, Jan Vanthienen, and Bart Baesens. "A multidimensional quality assessment of state-of-the-art process discovery algorithms using real-life event logs". In: Inf. Syst. 37.7 (2012), pp. 654-676 (cit. on pp. 26, 179).

[97] Jochen De Weerdt, Manu De Backer, Jan Vanthienen, and Bart Baesens. "A robust F-measure for evaluating discovered process models". In: IEEE Symposium on Computational Intelligence and Data Mining, CIDM'11. IEEE, 2011, pp. 148-155 (cit. on pp. 18, 41, 43, 57, 106).

[98] Jochen De Weerdt, Seppe K. L. M. vanden Broucke, Jan Vanthienen, and Bart Baesens. "Active Trace Clustering for Improved Process Discovery". In: IEEE Trans. Knowl. Data Eng. 25.12 (2013), pp. 2708-2720 (cit. on pp. 21, 86).

[99] Matthias Weidlich, Jan Mendling, and Mathias Weske. "Efficient Consistency Measurement Based on Behavioral Profiles of Process Models". In: IEEE Trans. Software Eng. 37.3 (2011), pp. 410-429 (cit. on pp. 168, 169).

[100] A.J.M.M. Weijters, Wil M. P. van der Aalst, and Ana Karla A. de Medeiros. "Process Mining with the Heuristics Miner-algorithm". In: BETA Working Paper Series. Vol. WP 166. 2006 (cit. on pp. 41, 182).

[101] Jan Martijn E. M. van der Werf, Boudewijn F. van Dongen, Cor A. J. Hurkens, and Alexander Serebrenik. "Process Discovery using Integer Linear Programming". In: Fundam. Inform. 94.3-4 (2009), pp. 387-412 (cit. on pp. 18, 57, 80, 83).

[102] Mathias Weske. Business Process Management - Concepts, Languages, Architectures, 2nd Edition. Springer, 2012, pp. I-XV, 1-403 (cit. on p. 41). 


\section{Publications}

\section{Precision}

[62] A Fresh Look at Precision in Process Conformance Jorge Munoz-Gama and Josep Carmona

Business Process Management, BPM 2010

Lecture Notes in Computer Science, vol. 6336, Springer, pp. 211-226.

[64] Enhancing precision in Process Conformance: Stability, confidence and severity Jorge Munoz-Gama and Josep Carmona

IEEE Symposium on Computational Intelligence and Data Mining, CIDM 2011

IEEE, pp. 184-191.

[63] A General Framework for Precision Checking

Jorge Munoz-Gama and Josep Carmona

International Journal of Innovative Computing, Information and Control (IJICIC),

vol.8 no.7 (B), July 2012

[18] Alignment Based Precision Checking

Arya Adriansyah, Jorge Munoz-Gama, Josep Carmona, Boudewijn F. van Dongen and Wil M. P. van der Aalst

Business Process Management Workshops 2012

Lecture Notes in Business Information Processing, vol. 132, Springer, pp. 137-149. 
[19] Measuring Precision of Modeled Behavior

Arya Adriansyah, Jorge Munoz-Gama, Josep Carmona, Boudewijn F. van Dongen and Wil M. P. van der Aalst

Information Systems and e-Business Management

Springer.

\section{Decomposition}

[66] Hierarchical Conformance Checking of Process Models Based on Event Logs Jorge Munoz-Gama, Josep Carmona and Wil M. P. van der Aalst

Petri Nets 2013

Lecture Notes in Computer Science, vol. 7927, Springer, pp. 291-310.

[65] Conformance Checking in the Large: Partitioning and Topology

Jorge Munoz-Gama, Josep Carmona and Wil M. P. van der Aalst

Business Process Management, BPM 2013

Lecture Notes in Computer Science, vol. 8094, Springer, pp. 130-145.

[67] Single-Entry Single-Exit Decomposed Conformance Checking Jorge Munoz-Gama, Josep Carmona and Wil M. P. van der Aalst Information Systems

vol. 46, December 2014, pp. 102-122, Elsevier.

[55] Decomposing Conformance Checking on Petri Nets with Data Massimiliano de Leoni, Jorge Munoz-Gama, Josep Carmona and Wil M.P. van der Aalst Cooperative Information Systems, CoopIS 2014

Lecture Notes in Computer Science, vol. 8841, Springer, pp. 3-20.

[29] Event-based Real-time Decomposed Conformance Analysis Seppe K.L.M. vanden Broucke, Jorge Munoz-Gama, Josep Carmona, Bart Baesens and Jan Vanthienen

Cooperative Information Systems, CoopIS 2014

Lecture Notes in Computer Science, vol. 8841, Springer, pp. 345-363. 


\section{Acknowledgements}

The first person I would like to thank is my advisor Josep Carmona. I would like to express my gratitude to him for his expertise, understanding, and patience. I appreciate his knowledge and skill (an 'oracle' to me), his determination and patience teaching me, his concept of ethics in the academic world, the hours of work spent together, and his comprehension in my darkest hours. From him I learned to be never satisfied with mediocrity, and to always give the best of myself. Thanks for having your office always open for me! On the other hand, he has a horrible taste in football.

Next, I would like to thank Wil van der Aalst for opening the door of the TU Eindhoven, making me feel a member of his group from minute one, and for sharing with me his views on the process mining field. It was a real privilege to work with him and I hope to keep collaborating in the future. I would like to extend my thanks to the rest of the people in Eindhoven, specially to Arya Adriansyah (I learned something new each second I spent working with him, and it is a clear example that two people with different cultures can get along really well), Massimiliano de Leoni (work and fun together make the conferences more interesting), Boudewijn van Dongen, Eric Verbeek, and the others. I also want to mention Ine van der Ligt and Riet van Buul for solving all the problems I brought them. To all of you, thanks for making my stay in Eindhoven memorable.

During my PhD I have had the privilege to work with great people from other research groups around the world. I would like to thank Isao Echizen from the NII for advising me, giving me the opportunity to work in a place so exotic for me like Japan. His creative mind and his practical vision of the security and the world in general is something remarkable. I would like to thank also Seppe vanden Broucke (hard-working researcher and greater person), Bart Baesens and Jan Vanthienen from the KU Leuven. I would also like to thank Artem Polyvyanyy for his advice and comments, and let's hope we finally meet in a conference one day. Some of these people have visited us in Barcelona, and I had the opportunity to work side by side with them: Joel Ribeiro, Thomas Stocker, and Xixi Lu. It has been a pleasure to have you here. Collaborating with Ernest Teniente and Montse Estañol is a clear evidence that in science there are more things in common between us than the ones keeping us apart.

The academic path is a road full of bumps. This is why I would like to thank all the people that made this work possible: the people in charge of the department, the university, 
the research projects, and the administrative tasks. Not to forget Ramon Xuriguera and Daniel Alonso, that never had a no for me when my deadlines where around the corner.

I would like to give a special mention to Marcos Sepúlveda and Mar Pérez-Sanagustín from the UC in Chile. They are the ones to blame for my next exciting adventure, and to make that transition millions of times more peaceful.

To enumerate all the friends that shared with me this period of my life would require another thesis: the friends of S108 (and S106, of course), The Netherlands, Japan, Bergen, Barcelona, Folloneres, China, Haskell, Castellón, San Francisco, Vic, Ragú, Gelida, Ibiza, Cantabria, Indonesia, Leuven, Italy, Iran, Curitiba, Ukraine, Russia, Porto, France, and all the others. Without you, I would have finished this thesis much earlier.

Finally, I would like my last thanks go to my parents Loly and Emilio, my brother Alex, and the rest of my family. I am truly grateful to have you by my side, in the good but also in the bad moments. This thesis is dedicated to you.

This work has been partly supported by Ministerio de Educación. 